Supporting Information for

\title{
Construction of Indoline/Indolenine Ring Systems by a Palladium-Catalyzed Intramolecular Dearomative Heck Reaction and the Subsequent Aza-semipinacol Rearrangement
}

\author{
Dong Gao and Lei Jiao* \\ Center of Basic Molecular Science (CBMS), Department of Chemistry, Tsinghua \\ University, Beijing 100084 (China) \\ E-mail: Leijiao@mail.tsinghua.edu.cn.
}

\section{Contents}

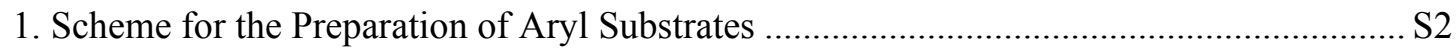

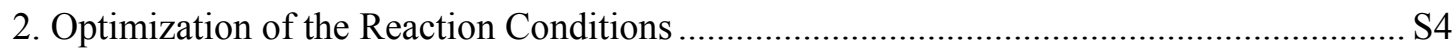

3. Stereospecificity of the Aza-Semipinacol Rearrangement .................................................. S8

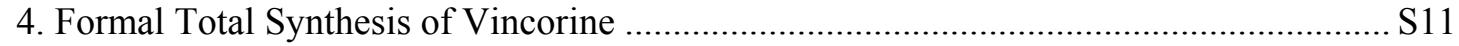

5. Scheme for the Preparation of the Alkenyl Substrates ....................................................... $\mathrm{S} 12$

6. X-ray Single Crystal Diffraction Analysis ..................................................................... S13

6.1 Crystallographic Data of Compound 4ha (CCDC 2052644) .................................... S13

6.2 Crystallographic Data of Compound 4hb (CCDC 2052643) .................................... S14

7. Energy Profile of the Aza-Semipinacol Rearrangement …………………..................... S15

7.1 Energy profiles for aza-semipinacol rearrangement (Figure 2a) ............................. S15

7.2 Energy profiles for the substituent effect (Figure 2b) ........................................... S15

7.3 Coordinates and energies of all optimized structures ................................................. S16

8. NMR Spectra for Synthesized Compounds .................................................................... S36

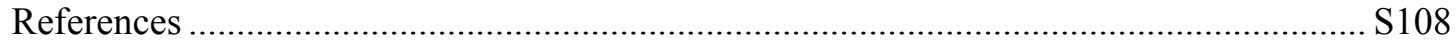




\section{Scheme for the Preparation of Aryl Substrates}<smiles>[R]c1ccc2[nH]c3c(c2c1)CC[NH2+]C3</smiles>

S1 $\left(R^{1}=H\right)$

$\mathrm{S} 11\left(\mathrm{R}^{1}=\mathrm{OMe}\right)$<smiles>[R10]c1ccc2[nH]c(C=Cc3cc([R])c([R])cc3Br)c(CCNC(=O)O[Na])c2c1</smiles>

S3 $\left(R^{1}, R^{2}, R^{3}=H\right)$
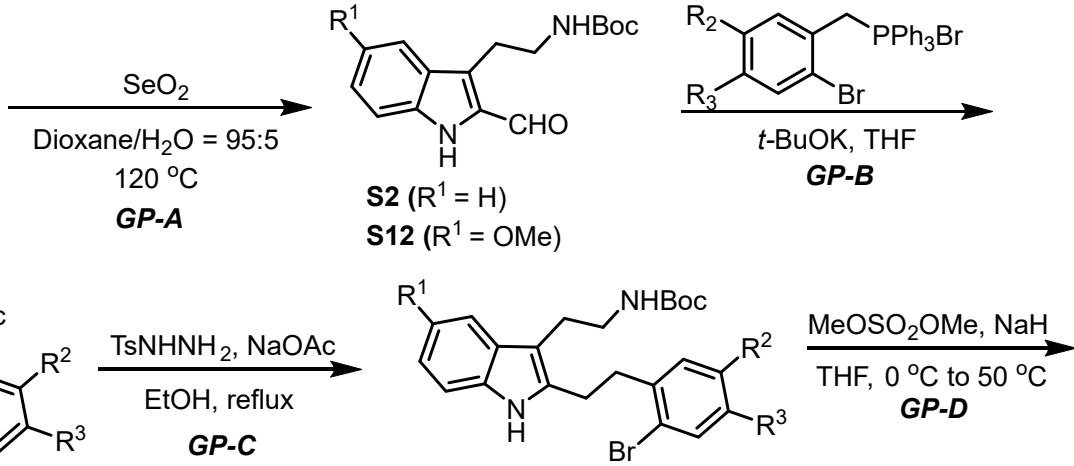

S4 $\left(R^{1}, R^{2}, R^{3}=H\right)$<smiles>[R]c1ccc2c(c1)c(CCNC(=O)O)c(CCc1cc([R])c([R])cc1Br)n2C</smiles>

1a $\left(R^{1}, R^{2}, R^{3}=H\right)$

1i $\left(R^{1}, R^{3}=H, R^{2}=C F_{3}\right)$

1d $\left(\mathrm{R}^{1}, \mathrm{R}^{3}=\mathrm{H}, \mathrm{R}^{2}=\mathrm{OMe}\right) \quad$ 1j $\left(\mathrm{R}^{1}=\mathrm{H}, \mathrm{R}^{2}, \mathrm{R}^{3}=-\mathrm{CH}=\mathrm{CH}-\mathrm{CH}=\mathrm{CH}-\right)$

(1) $\quad 1 e\left(R^{1}=H, R^{2}, R^{3}=-O-C_{2}-O-\right) \quad 1 m\left(R^{1}=O M e, R^{2}, R^{3}=H\right)$

1f $\left(R^{1}, R^{2}=H, R^{3}=M e\right)$

$\lg \left(R^{1}, R^{3}=H, R^{2}=F\right)$

1h $\left(R^{1}, R^{2}=H, R^{3}=C l\right)$<smiles>Cn1c(CCc2ccccc2Br)c(CCNC(=O)OC(C)(C)C)c2ccccc21</smiles>

1a

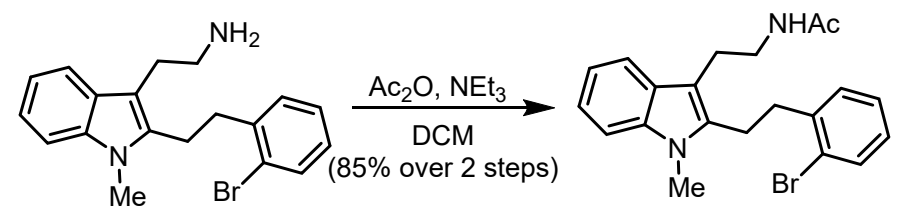

$1 b$<smiles>Cn1c(CCc2ccccc2Br)c(CCNS)c2ccccc21</smiles>

$1 c$<smiles>CC(C)(C)OCCNCc1c(CCc2ccccc2Br)[nH]c2ccccc12</smiles>

S4

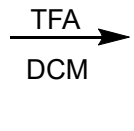

S5

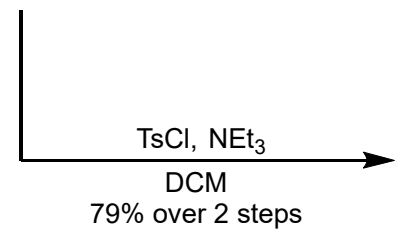<smiles>O=C(NCCc1c(CCc2ccccc2Br)[nH]c2ccccc12)OCc1ccccc1</smiles>

s9

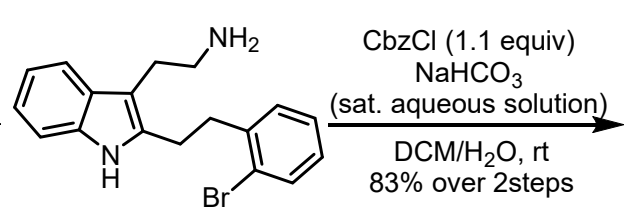

58

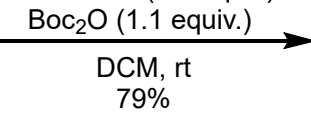

GP-E
11<smiles>CC(=O)NCCc1c(CCc2ccccc2Br)n(C(=O)OCc2ccccc2)c2ccccc12</smiles>

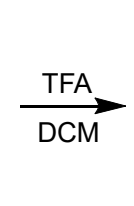

SchemeS1 General Procedures for the synthesis of aryl bromide 1a-j and 11-m 
<smiles>O=Cc1[nH]c2ccccc2c1CCNCCCO</smiles>

S2

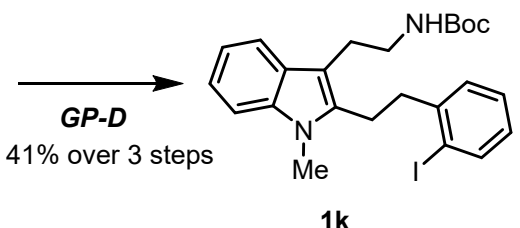

(5)

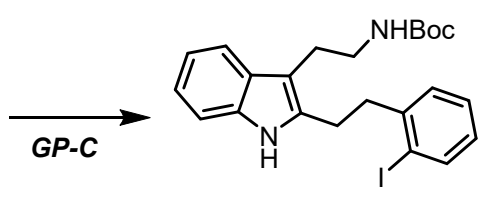

s7

SchemeS2 Procedure for the synthesis of aryl iodide $\mathbf{1 k}$<smiles>Cc1c(CCc2ccccc2Br)[nH]c2cc(CCc3ccccc3CCc3ccccc3Br)ccc12</smiles><smiles>Cc1c(CCc2ccccc2Br)n(C(=O)OC(C)(C)C)c2ccccc12</smiles>

1n<smiles>CC#Cc1ccccc1C(=O)O[Mg]</smiles>

S15<smiles></smiles>

10

SchemeS3 Procedure for the synthesis of aryl bromide 1n-o 


\section{Optimization of the Reaction Conditions}

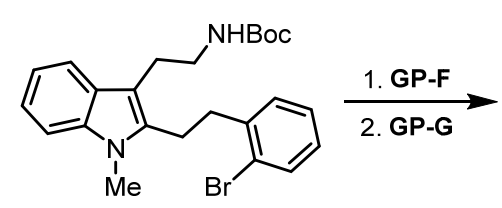

1a<smiles>CN1c2ccccc2C23CCCCC12NCC3</smiles>

3aa

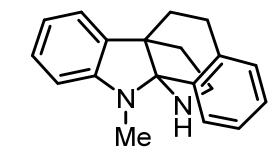

$3 a b$

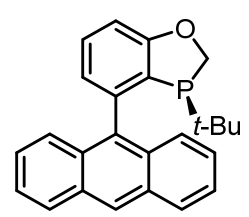

L3<smiles>Oc1ccc2ccccc2c1-c1c(OP(Oc2cccc3ccccc23)N2c3ccccc3C=Cc3ccccc32)ccc2ccccc12</smiles>

L8

Table 1, entry 3: Synthesized according to GP-F and GP-G using 1a (45 mg, $0.1 \mathrm{mmol}, 1.0$ equiv.) as substrate, $\mathbf{L 3}(2.8 \mathrm{mg}, 0.0075 \mathrm{mmol}, 7.5 \mathrm{~mol} \%)$ as chiral ligand, DCE (1.0 mL) as solvent. The reaction was conducted at $120{ }^{\circ} \mathrm{C}$ to afford 3aa $(10.7 \mathrm{mg}, 39 \%$ yield over 2 steps, $22 \%$ ee) as a yellow oil and 3ab (2.0 mg, $8 \%$ yield over 2 steps, $21 \%$ ee) as a yellow oil. Reaction time for GPF: $72 \mathrm{~h}$; Reaction time for GP-G: $1 \mathrm{~h}$. The ee of compound 3aa and 3ab was determined by HPLC using an IA column $\left(n\right.$-hexane $/ i-\mathrm{PrOH}=100 / 5$, flow rate $=1.0 \mathrm{~mL} / \mathrm{min}$. For 3aa: $t_{\text {major }}=6.4 \mathrm{~min}$, $t_{\text {minor }}=7.3 \mathrm{~min}$; For $3 \mathbf{a b}: t_{\text {major }}=5.8 \mathrm{~min}, t_{\text {minor }}=5.1 \mathrm{~min}$ ). 
HPLC Data for 3aa

Racemic 3aa:

mAU

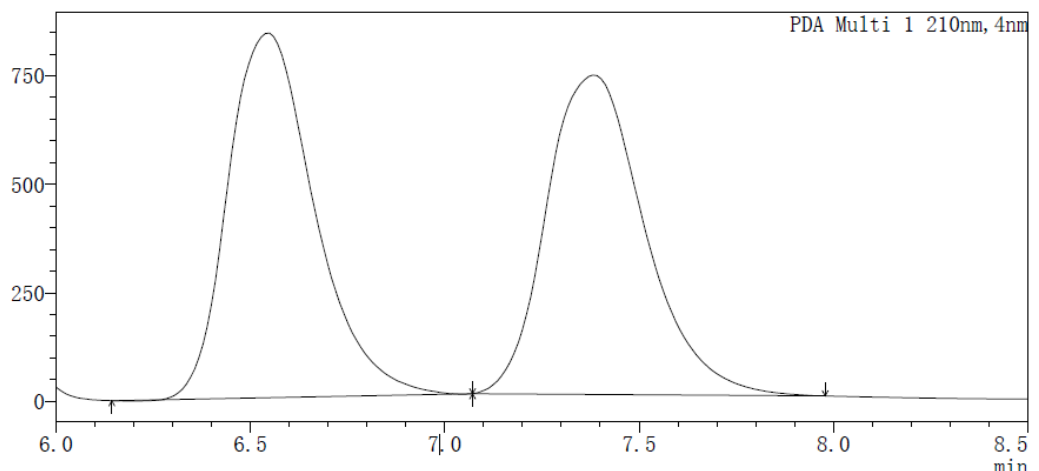

〈Peak Results〉

PDA Ch1 210nm

\begin{tabular}{|c|c|c|c|c|}
\hline Index & Time/min & Height/mAU & Quantity/Area & Area $\% / \%$ \\
\hline 1 & 6.540 & 839138 & 12952135 & 50.099 \\
\hline 2 & 7.378 & 73516 & 12900 & \\
\hline
\end{tabular}

Enantioenriched 3aa:

mAU

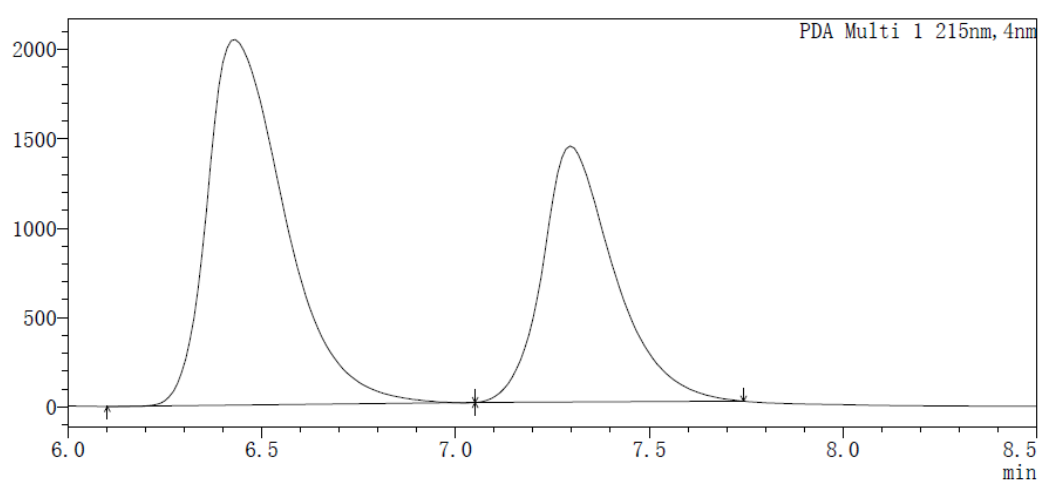

〈Peak Results〉

PDA Ch1 215nm

\begin{tabular}{|c|c|c|c|c|}
\hline Index & Time/min & Height/mAU & Quantity/Area & Area $\% / \%$ \\
\hline 1 & 6.424 & 2044011 & 28534939 & 60.541 \\
\hline 2 & 7.291 & 1432522 & 18598569 & 39.459 \\
\hline
\end{tabular}




\section{HPLC Data for 3ab}

Racemic 3ab:

$\mathrm{mAU}$

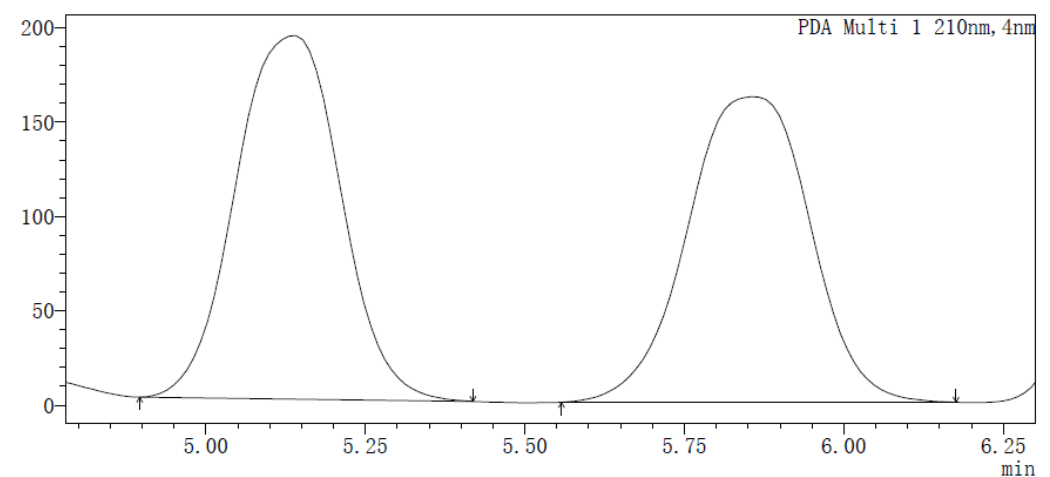

〈Peak Results

PDA Ch1 210nm

\begin{tabular}{|c|c|c|c|c|}
\hline Index & Time/min & Height/mAU & Quantity/Area & Area \%/\% \\
\hline 1 & 5.132 & 192626 & 2206302 & 50.474 \\
\hline 2 & 5.852 & 161929 & 2164862 & 49.526 \\
\hline
\end{tabular}

\section{Enantioenriched 3ab:}

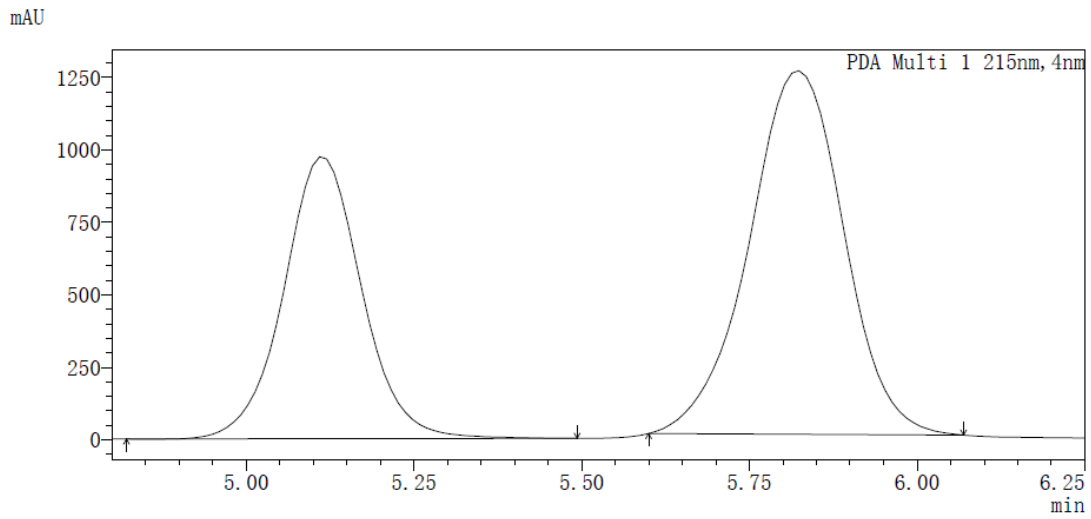

〈Peak Results〉

PDA Ch1 $215 \mathrm{~nm}$
\begin{tabular}{|c|c|c|c|c|}
\hline Index & Time/min & Height/mAU & Quantity/Area & Area $\% / \%$ \\
\hline 1 & 5.107 & 973626 & 7886369 & 38.969 \\
\hline
\end{tabular}

\begin{tabular}{|c|c|c|c|c|}
\hline 1 & 5.107 & 973626 & 7886369 & 38.969 \\
\hline 2 & 5.817 & 1254869 & 12351368 & 61.031 \\
\hline
\end{tabular}


Table 1, entry 8: Synthesized according to GP-F and GP-G using 1 a (42 mg, $0.09 \mathrm{mmol}, 1.0$ equiv.) as substrate, $\mathbf{L 3}$ (3.6 $\mathrm{mg}, 0.0069 \mathrm{mmol}, 7.5 \mathrm{~mol} \%)$ as chiral ligand, Toluene $(1.0 \mathrm{~mL})$ as solvent. The reaction was conducted at $120{ }^{\circ} \mathrm{C}$ to afford $3 \mathrm{aa}(8.1 \mathrm{mg}, 33 \%$ yield over 2 steps, $10 \%$ ee) as a yellow oil and 3ab ( $4.8 \mathrm{mg}, 19 \%$ yield over 2 steps, $11 \%$ ee) as a yellow oil. Reaction time for GPF: $48 \mathrm{~h}$; Reaction time for GP-G: $1 \mathrm{~h}$. The ee of compound 3aa and 3ab was determined by HPLC using an IA column $\left(n\right.$-hexane $/ i-\mathrm{PrOH}=95 / 5$, flow rate $=1.0 \mathrm{~mL} / \mathrm{min}$. For 3aa: $t_{\text {major }}=6.4 \mathrm{~min}$, $t_{\text {minor }}=7.3 \mathrm{~min}$; For $3 \mathbf{a b}: t_{\text {major }}=5.8 \mathrm{~min}, t_{\text {minor }}=5.1 \mathrm{~min}$ ).

HPLC Data for 3aa:

mAU

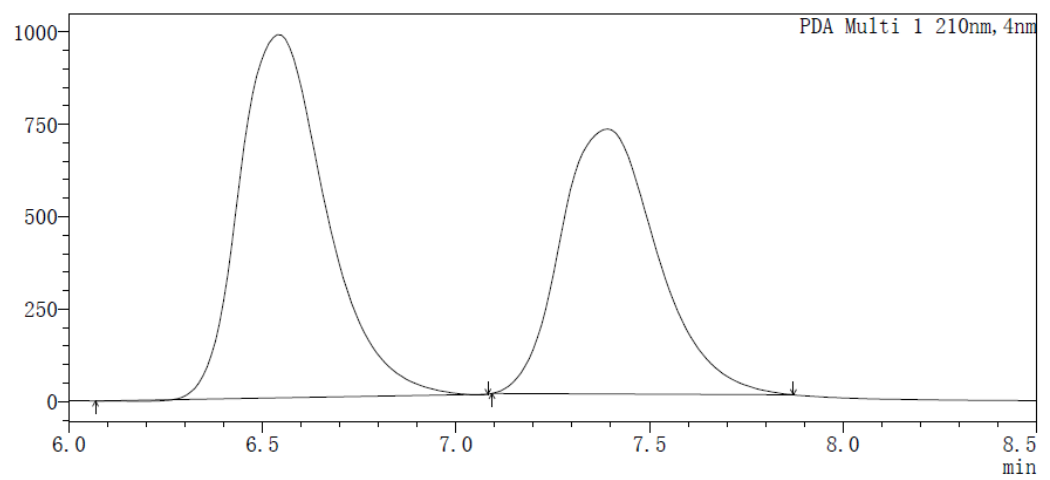

〈Peak Results〉

PDA Ch1 210nm

\begin{tabular}{|c|c|c|c|c|}
\hline Index & Time/min & Height/mAU & Quantity/Area & Area \%/\% \\
\hline 1 & 6.537 & 982112 & 15282058 & 55.048 \\
\hline 2 & 7.386 & 717302 & 12479250 & 44.952 \\
\hline
\end{tabular}

HPLC Data for 3ab:

$\mathrm{maU}$

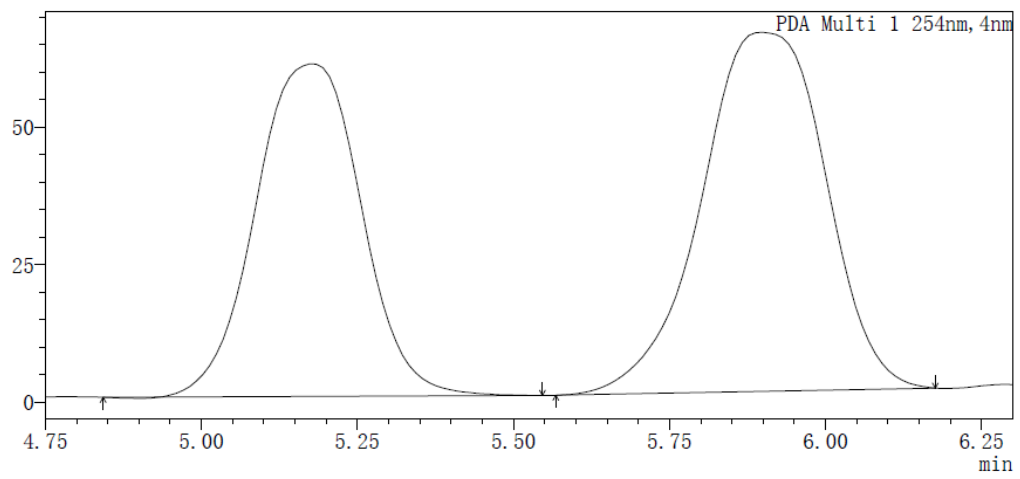

〈Peak Results〉

PDA Ch1 254nm

PDA Ch1 $254 \mathrm{~nm}$
\begin{tabular}{|c|c|c|c|c|}
\hline Index & Time/min & Height/mAU & Quantity/Area & Area $\% / \%$ \\
\hline 1 & 5.173 & 60814 & 714394 & 44.035 \\
\hline 2 & 5.892 & 65679 & 907934 & 55.965 \\
\hline
\end{tabular}




\section{Stereospecificity of the Aza-Semipinacol Rearrangement}

\section{HPLC Data for 2n}

Racemic 2n:

$\mathrm{mAU}$

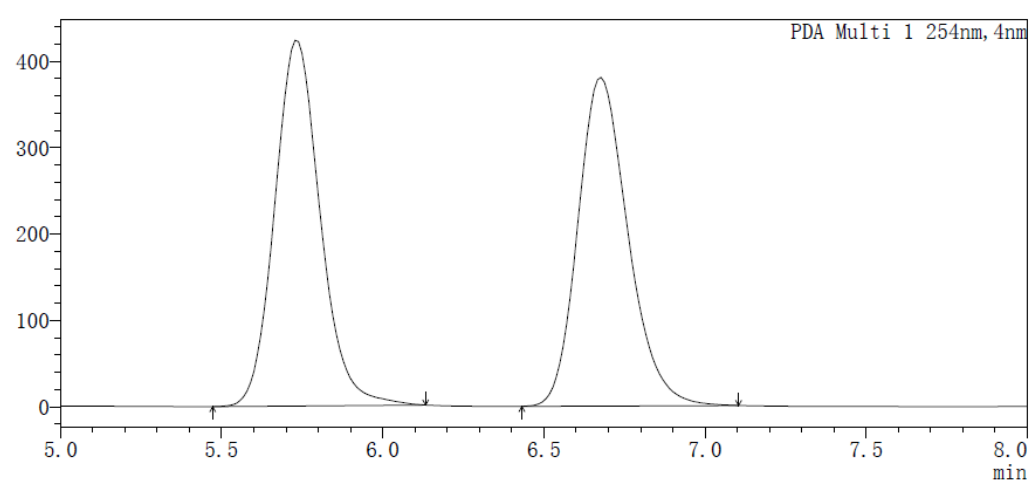

〈Peak Results

PDA Ch1 254nm

PDA Ch1 $254 \mathrm{~nm}$
\begin{tabular}{|c|c|c|c|c|}
\hline Index & Time/min & Height/mAU & Quantity/Area & Area $\% / \%$ \\
\hline 1 & 5.732 & 423158 & 4168776 & 50.333 \\
\hline 2 & 6.675 & 380548 & 4113595 & 49.667 \\
\hline
\end{tabular}

Enantioenriched 2n:

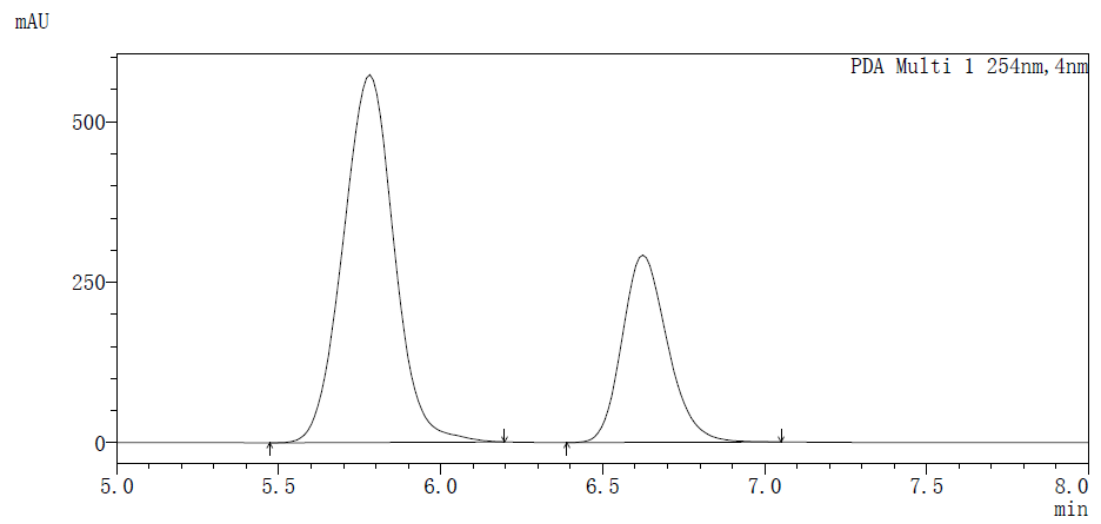

〈Peak Results〉

PDA Ch1 $254 \mathrm{~nm}$
\begin{tabular}{|c|c|c|c|c|}
\hline Index & Time/min & Height/mAU & Quantity/Area & Area $\% / \%$ \\
\hline 1 & 5.780 & 572902 & 6282138 & 68.824 \\
\hline 2 & 6.624 & 291956 & 2845664 & 31.176 \\
\hline
\end{tabular}


HPLC Data for 3na

Racemic 3na:

$\mathrm{mAU}$

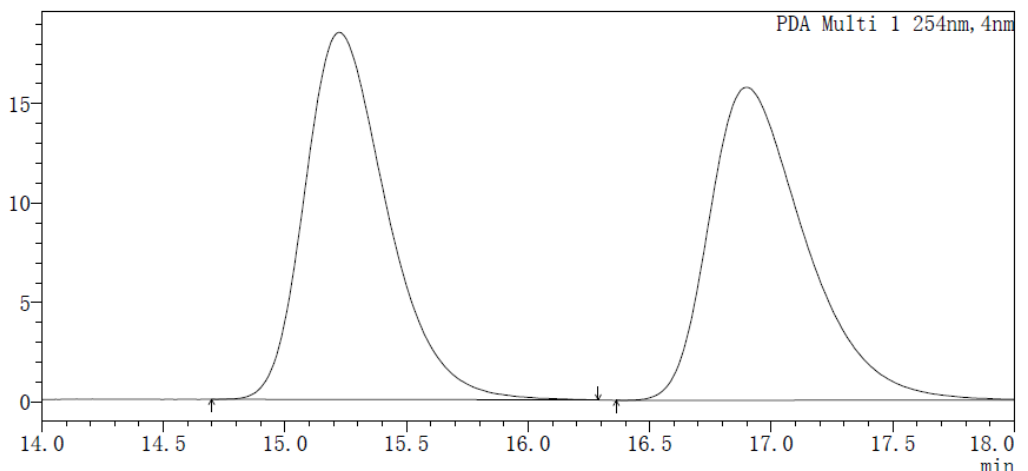

〈Peak Results〉

PDA Ch1 $254 \mathrm{~nm}$
\begin{tabular}{|c|c|c|c|c|}
\hline Index & Time/min & Height/mAU & Quantity/Area & Area \%/\% \\
\hline 1 & 15.224 & 18465 & 435955 & 50.145 \\
\hline 2 & 16.899 & 15735 & 433433 & 49.855 \\
\hline
\end{tabular}

Enantioenriched 3na:

mAU

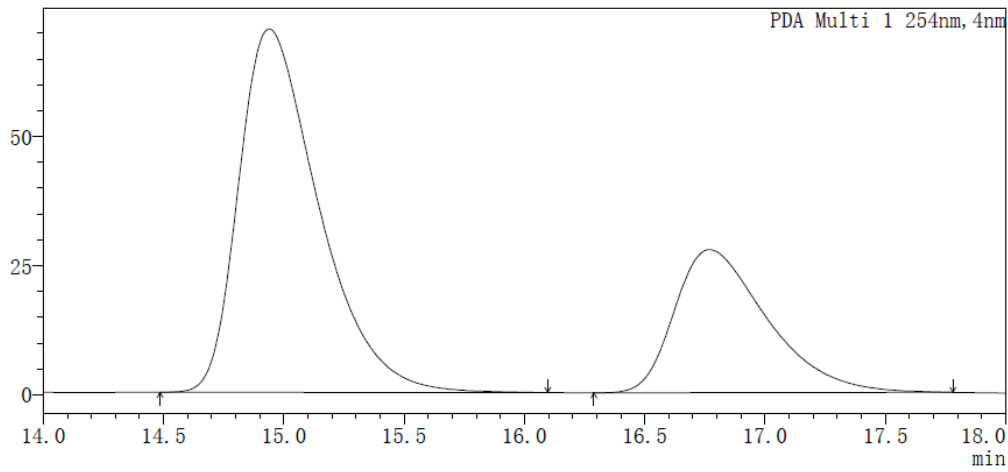

〈Peak Results〉

PDA Ch1 254nm

\begin{tabular}{|c|c|c|c|c|} 
PDA Ch1 & \multicolumn{1}{|c|}{ Inm } \\
\hline Index & Time/min & Height/mAU & Quantity/Area & Area $\% / \%$ \\
\hline 1 & 14.939 & 70329 & 1628558 & 68.702 \\
\hline 2 & 16.770 & 27708 & 741919 & 31.298 \\
\hline
\end{tabular}


HPLC Data for 3nb

Racemic 3nb:

$\mathrm{mAU}$

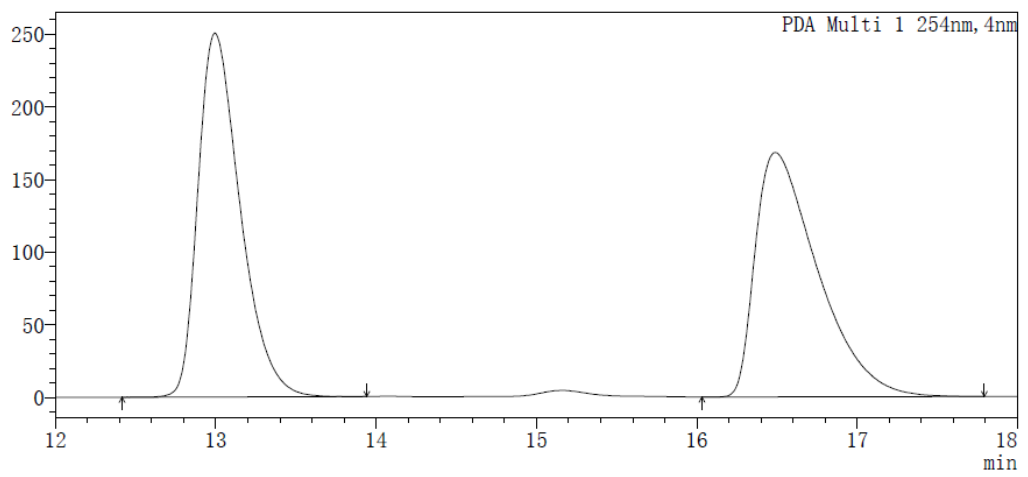

〈Peak Results

PDA Ch1 254nm

\begin{tabular}{|c|c|c|c|c|}
\hline Index & Time/min & Height/mAU & Quantity/Area & Area \%/\% \\
\hline 1 & 12.994 & 250383 & 4581662 & 50.130 \\
\hline 2 & 16.489 & 168145 & 4557872 & 49.870 \\
\hline
\end{tabular}

Enantioenriched 3nb:

mAU

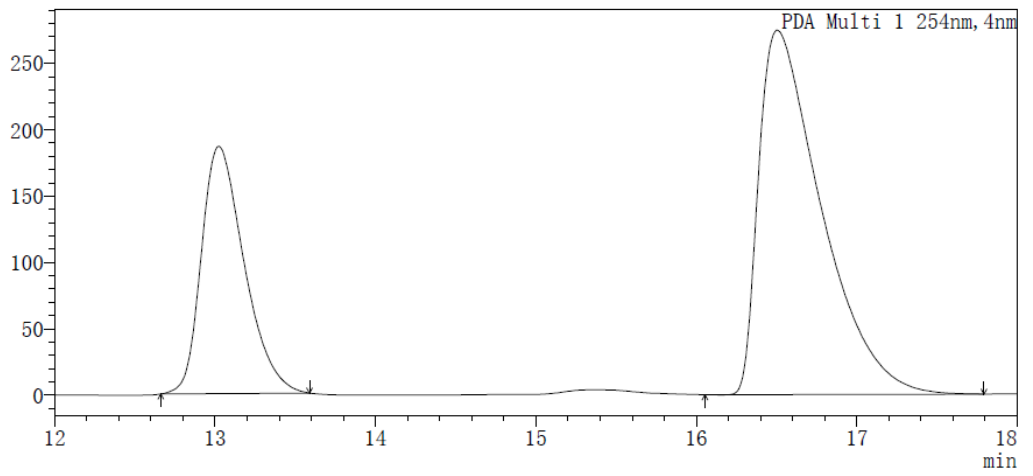

〈Peak Results〉

PDA Ch1 254nm

\begin{tabular}{|c|c|c|c|c|}
\hline Index & Time/min & Height/mAU & Quantity/Area & Area \%/\% \\
\hline 1 & 13.023 & 186359 & 3461086 & 31.769 \\
\hline 2 & 16.505 & 274408 & 7433371 & 68.231 \\
\hline
\end{tabular}




\section{Formal Total Synthesis of Vincorine}

\section{HPLC Data for 10}

Racemic 10:

$\mathrm{mAU}$

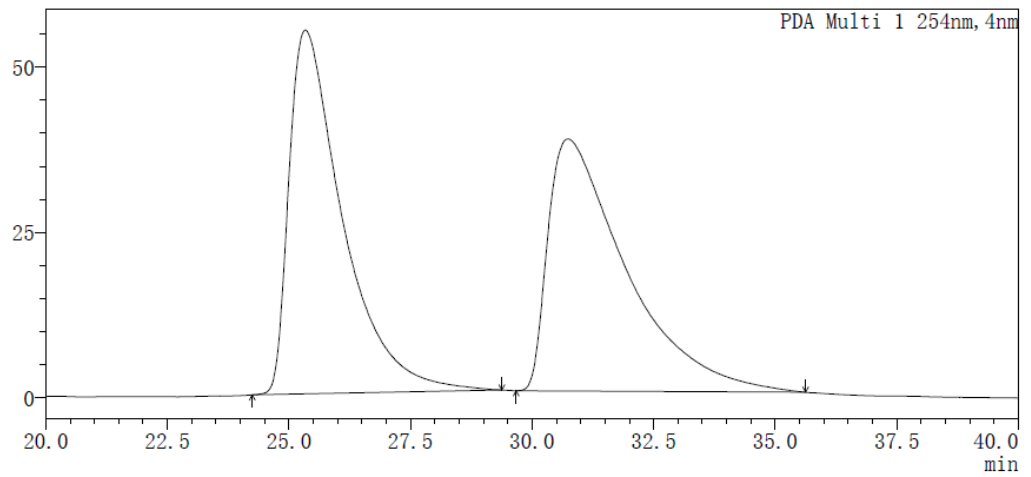

$\langle$ Peak Results>

PDA Ch1 254nm

\begin{tabular}{|c|c|c|c|c|}
\hline Index & Time/min & Height/mAU & Quantity/Area & Area \%/\% \\
\hline
\end{tabular}

\begin{tabular}{c|c|c|c|c|} 
Index & Time/min & Height/mAU & Quantity/Area & Area \%/\% \\
\hline 1 & 25.339 & 54971 & 4221288 & 49.825 \\
\hline 2 & 30.740 & 38083 & 4250906 & 50.175
\end{tabular}

Enantioenriched 10:

mAU

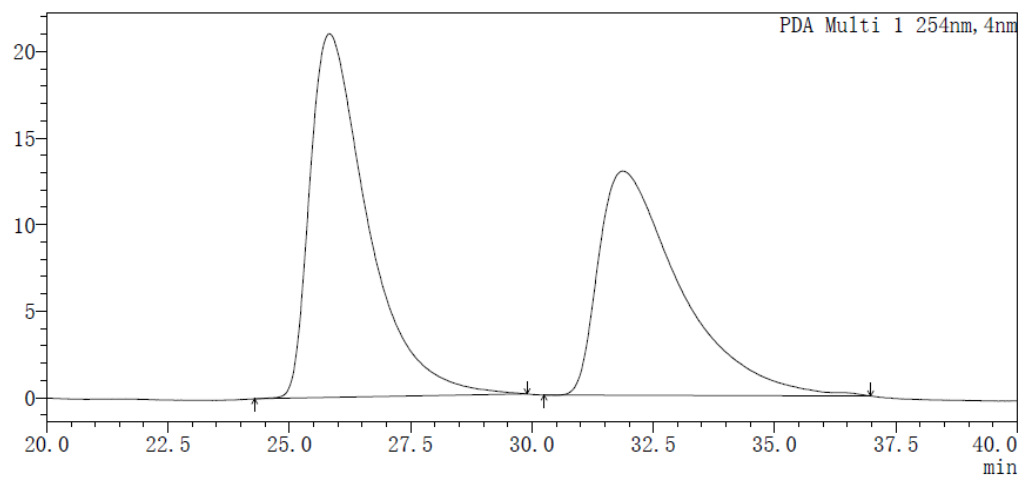

〈Peak Results〉

PDA Ch1 $254 \mathrm{~nm}$
\begin{tabular}{|c|c|c|c|c|}
\hline Index & Time/min & Height/mAU & Quantity/Area & Area $\% / \%$ \\
\hline 1 & 25.818 & 21013 & 1746860 & 53.489 \\
\hline 2 & 31.872 & 12956 & 1518942 & 46.511 \\
\hline
\end{tabular}




\section{Scheme for the Preparation of the Alkenyl Substrates}
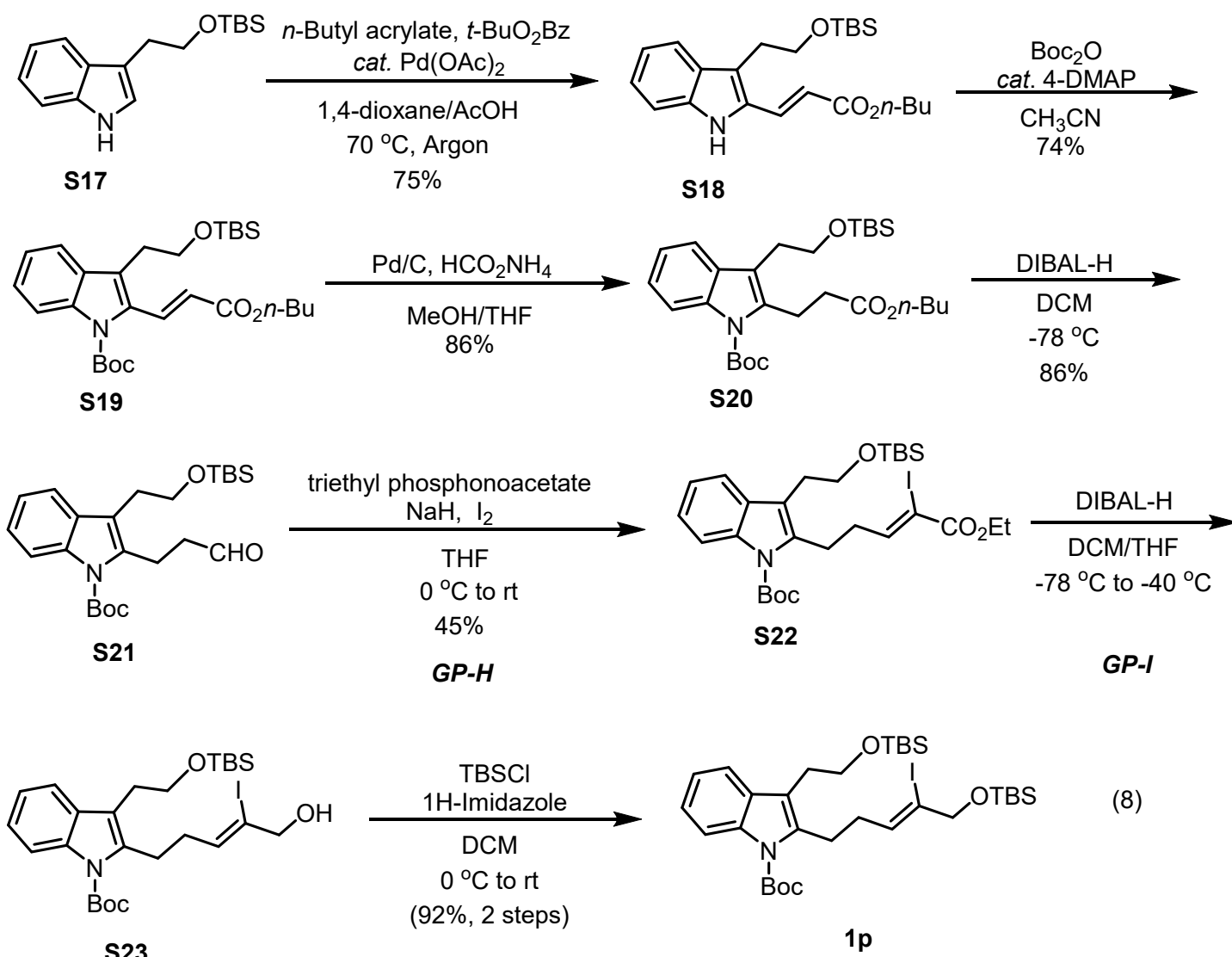<smiles>[B]n1c(CC(CC(=O)NCCCCC(=O)O)C(C)=O)c(C)c2ccccc21</smiles>

S24

S25<smiles>[B]n1c(CC(CC2=C(Br)CCCC2)(C(C)=O)C(=O)OC)c(C)c2ccccc21</smiles>

$1 q$

SchemeS4 Procedure for the synthesis of alkenyl substrates $\mathbf{1 p - q}$ 


\section{X-ray Single Crystal Diffraction Analysis}

\subsection{Crystallographic Data of Compound 4ha (CCDC 2052644)}

Crystal compound 4 ha was obtained by adding $n$-hexane to its dichloromethane solution and then stilling for several days.

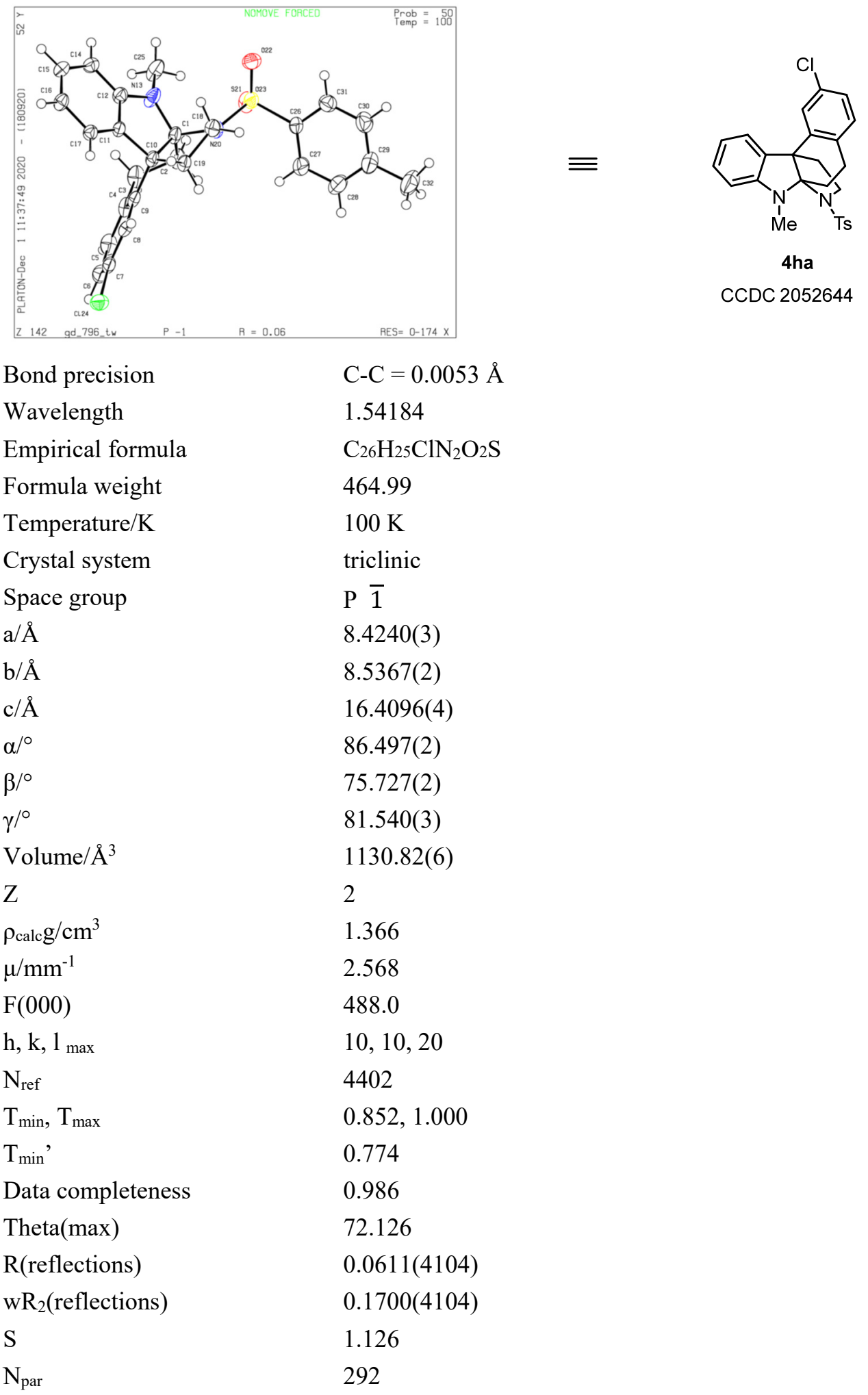




\subsection{Crystallographic Data of Compound 4hb (CCDC 2052643)}

Crystal compound $\mathbf{4 h b}$ was obtained by adding $n$-hexane to its dichloromethane solution and then stilling for several days.

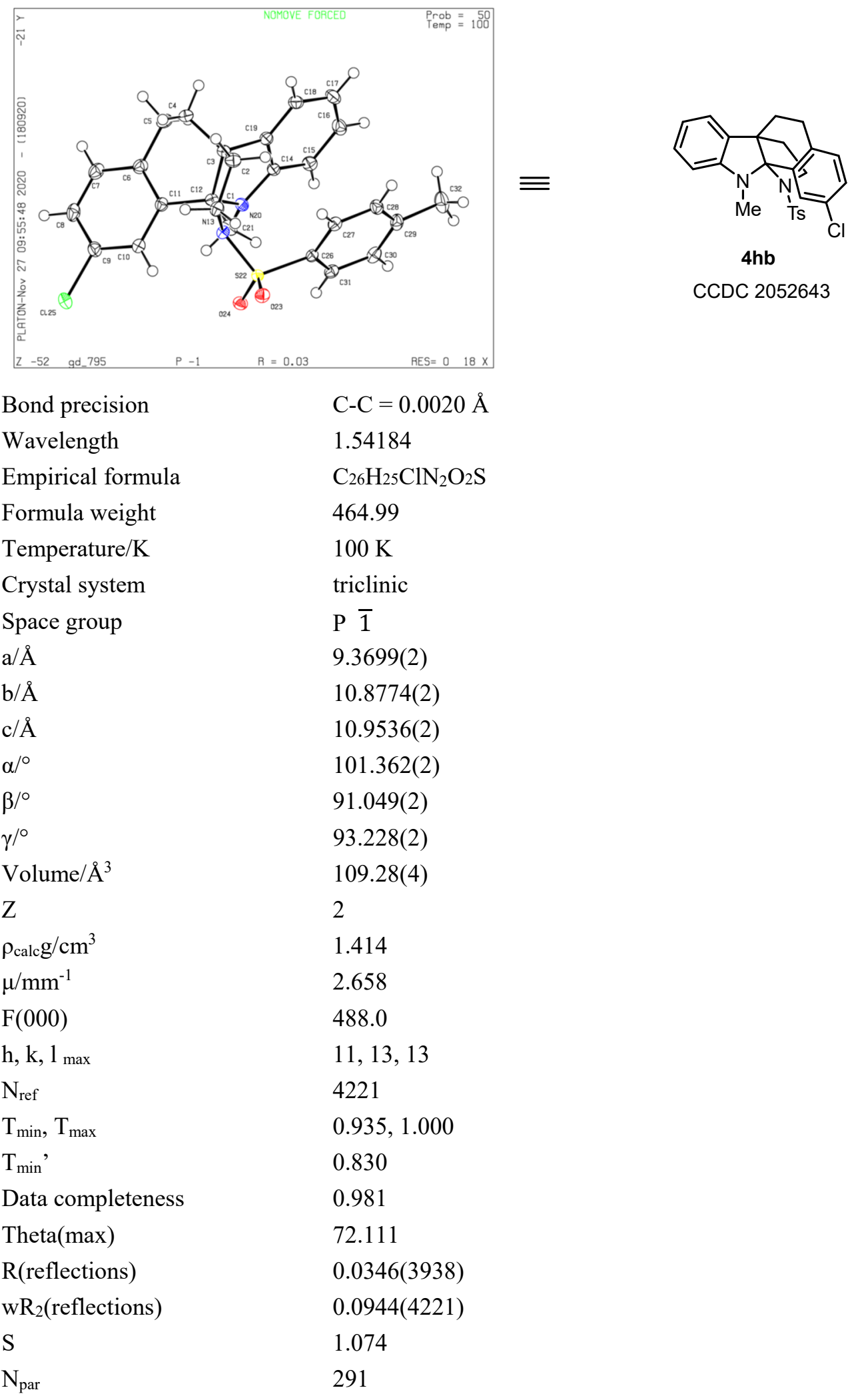




\section{Energy Profile of the Aza-Semipinacol Rearrangement}

7.1 Energy profiles for aza-semipinacol rearrangement (Figure 2a)

Energy data ${ }^{a}$ of 2 r:

\begin{tabular}{|c|c|c|c|}
\hline & $\mathbf{Z P E}^{b}$ & $\mathbf{E}(\mathbf{M 0 6 - 2 X})^{c}$ & $\begin{array}{c}\text { Imaginary } \\
\text { frequency }\end{array}$ \\
\hline IN1 & 0.270504 & -751.249216098 & \\
\hline TS1a & 0.270892 & -751.225864796 & $-405.60 i$ \\
\hline IN1a & 0.273030 & -751.267534060 & \\
\hline TS1b & 0.270170 & -751.224409807 & $-418.77 i$ \\
\hline IN1b & 0.274653 & -751.274011991 & \\
\hline
\end{tabular}

a. in Hartree

b. zero-point correction

c. single-point energies at the level of M06-2X/6-311++G(2df,2p)

\subsection{Energy profiles for the substituent effect (Figure 2b)}

Energy data ${ }^{a}$

\begin{tabular}{|c|c|c|c|c|}
\hline \multirow{2}{*}{ Substrate } & TS & $\mathbf{Z P E}^{b}$ & $\mathbf{E}(\mathbf{M 0 6 - 2 X})^{c}$ & $\begin{array}{c}\text { Imaginary } \\
\text { frequency }\end{array}$ \\
\hline \multirow{2}{*}{$\mathbf{2 s}$} & TS1a & 0.268256 & -1088.29072839 & $-430.33 i$ \\
\cline { 2 - 5 } & TS1b & 0.267511 & -1088.29118893 & $-423.81 i$ \\
\hline \multirow{2}{*}{$2 \mathbf{t}$} & TS1a & 0.258665 & -1210.82698583 & $-418.36 i$ \\
\cline { 2 - 5 } & TS1b & 0.258073 & -1210.82683744 & $-421.94 i$ \\
\hline \multirow{2}{*}{$2 \mathbf{u}$} & TS1a & 0.295253 & -790.535632176 & $-397.53 i$ \\
\cline { 2 - 5 } & TS1b & 0.294752 & -790.533921428 & $-416.55 i$ \\
\hline \multirow{2}{*}{$2 \mathbf{v}$} & TS1a & 0.261245 & -850.471551213 & $-396.99 i$ \\
\cline { 2 - 5 } & TS1b & 0.260673 & -850.470225868 & $-422.75 i$ \\
\hline \multirow{2}{*}{$\mathbf{2 w}$} & TS1a & 0.299923 & -865.751641592 & $-330.81 i$ \\
\cline { 2 - 5 } & TS1b & 0.299033 & -865.747499472 & $-417.70 i$ \\
\hline \multirow{2}{*}{$\mathbf{2 x}$} & TS1a & 0.282601 & -939.767050250 & $-349.86 i$ \\
\cline { 2 - 5 } & TS1b & 0.281461 & -939.764135330 & $-421.04 i$ \\
\hline \multirow{2}{*}{$\mathbf{2 y}$} & TS1a & 0.314149 & -904.845401119 & $-368.53 i$ \\
\cline { 2 - 5 } & TS1b & 0.313333 & -904.853663610 & $-416.85 i$ \\
\hline \multirow{2}{*}{$\mathbf{2 z}$} & TS1a & 0.342090 & -943.395494774 & $-386.21 i$ \\
\cline { 2 - 5 } & TS1b & 0.340953 & -943.391233110 & $-385.44 i$ \\
\hline
\end{tabular}

a. in Hartree

b. zero-point correction

c. single-point energies at the level of M06-2X/6-311++G(2df,2p) 
7.3 Coordinates and energies of all optimized structures

\section{2r-IN1}

\begin{tabular}{|c|c|c|c|}
\hline $\mathrm{C}$ & 4.19662400 & 0.55683400 & -0.87517700 \\
\hline $\mathrm{C}$ & 4.20800600 & -0.78867400 & -0.36781000 \\
\hline $\mathrm{C}$ & 3.10315100 & -1.39297700 & 0.18675300 \\
\hline $\mathrm{C}$ & 1.90836300 & -0.62967300 & 0.24468700 \\
\hline $\mathrm{C}$ & 1.87595900 & 0.73464300 & -0.25603200 \\
\hline $\mathrm{C}$ & 3.05965100 & 1.31294900 & -0.82462600 \\
\hline $\mathrm{N}$ & 0.70887500 & -0.97264900 & 0.71766400 \\
\hline $\mathrm{C}$ & -0.23539200 & 0.15804000 & 0.59695700 \\
\hline $\mathrm{C}$ & 0.60625100 & 1.22723300 & -0.06098000 \\
\hline $\mathrm{C}$ & -0.81924000 & 0.60433300 & 1.99271200 \\
\hline $\mathrm{C}$ & -2.28788500 & 1.01724400 & 1.73058800 \\
\hline $\mathrm{C}$ & -2.63889100 & 0.30489000 & 0.44546000 \\
\hline $\mathrm{C}$ & -1.49785700 & -0.18372900 & -0.20008400 \\
\hline $\mathrm{C}$ & -1.57429900 & -0.84992800 & -1.42442700 \\
\hline $\mathrm{C}$ & 0.07833200 & 2.57167900 & -0.40739700 \\
\hline $\mathrm{C}$ & -2.83380400 & -1.04131800 & -1.99859300 \\
\hline $\mathrm{C}$ & -3.98389200 & -0.56168600 & -1.35734000 \\
\hline $\mathrm{C}$ & -3.89459300 & 0.11404200 & -0.13678400 \\
\hline $\mathrm{H}$ & 5.10933200 & 0.96254100 & -1.29740100 \\
\hline $\mathrm{H}$ & 5.13654800 & -1.34928500 & -0.42516300 \\
\hline $\mathrm{H}$ & 3.14949900 & -2.40659700 & 0.56696000 \\
\hline $\mathrm{H}$ & 3.04418500 & 2.32955000 & -1.20249200 \\
\hline $\mathrm{H}$ & -0.21589100 & 1.38725400 & 2.45782000 \\
\hline $\mathrm{H}$ & -0.79283900 & -0.26404500 & 2.65583700 \\
\hline $\mathrm{H}$ & -2.93650100 & 0.73569400 & 2.56596500 \\
\hline $\mathrm{H}$ & -2.38429200 & 2.10350400 & 1.61241800 \\
\hline $\mathrm{H}$ & -0.68226000 & -1.22116000 & -1.92182800 \\
\hline $\mathrm{H}$ & -0.22249200 & 3.11438100 & 0.49790300 \\
\hline $\mathrm{H}$ & -0.81704800 & 2.48274100 & -1.03334100 \\
\hline $\mathrm{H}$ & 0.81724700 & 3.17660600 & -0.93454700 \\
\hline $\mathrm{H}$ & -2.92105700 & -1.56458700 & -2.94539200 \\
\hline $\mathrm{H}$ & -4.95639000 & -0.71985300 & -1.81297800 \\
\hline $\mathrm{H}$ & -4.79234200 & 0.47862000 & 0.35402500 \\
\hline $\mathrm{C}$ & 0.35259200 & -2.26258400 & 1.29918600 \\
\hline $\mathrm{H}$ & -0.73014300 & -2.38343500 & 1.25334400 \\
\hline $\mathrm{H}$ & 0.81057100 & -3.06862100 & 0.72173000 \\
\hline $\mathrm{H}$ & 0.68329400 & -2.33267200 & 2.34093500 \\
\hline
\end{tabular}

\section{2r-TS1a}

$\mathrm{C}$

$-3.60984600$

$-1.37038400$

0.57407300

$\mathrm{C}$

$-3.91729000$

$-0.60512400$

$-0.57121300$

$\mathrm{C}$

$-3.03634000$

0.34410000

$-1.08205400$ 


$\begin{array}{lrrr}\mathrm{C} & -1.82653500 & 0.52539300 & -0.39885900 \\ \mathrm{C} & -1.49470200 & -0.25252000 & 0.73427000 \\ \mathrm{C} & -2.39598600 & -1.20710000 & 1.23048000 \\ \mathrm{~N} & -0.79073500 & 1.41431400 & -0.66457700 \\ \mathrm{C} & 0.25200300 & 1.17137900 & 0.23175400 \\ \mathrm{C} & -0.18063100 & 0.16552500 & 1.18555000 \\ \mathrm{C} & 1.35057800 & 2.18338100 & 0.50644300 \\ \mathrm{C} & 2.66441800 & 1.41421200 & 0.79389100 \\ \mathrm{C} & 2.47712000 & 0.05158300 & 0.17460200 \\ \mathrm{C} & 1.13575600 & -0.32618400 & -0.04687000 \\ \mathrm{C} & 0.80825400 & -1.47759900 & -0.77996500 \\ \mathrm{C} & 0.29202500 & -0.05998900 & 2.59568100 \\ \mathrm{H} & -4.32943800 & -2.09540500 & 0.93888500 \\ \mathrm{H} & -4.86955700 & -0.76006400 & -1.06873300 \\ \mathrm{H} & -3.29118000 & 0.91877700 & -1.96513200 \\ \mathrm{H} & -2.15465500 & -1.80327100 & 2.10472200 \\ \mathrm{H} & 1.48211900 & 2.82514700 & -0.36684300 \\ \mathrm{H} & 1.05034800 & 2.82818100 & 1.33864800 \\ \mathrm{H} & 2.85975400 & 1.34230500 & 1.87005600 \\ \mathrm{H} & 3.52706400 & 1.92965600 & 0.36342800 \\ \mathrm{H} & -0.34961800 & 0.51634400 & 3.27231800 \\ \mathrm{H} & 0.19982400 & -1.11519500 & 2.86474400 \\ \mathrm{H} & 1.32293600 & 0.24423300 & 2.76426500 \\ \mathrm{C} & -0.67151100 & 2.13005200 & -1.93200100 \\ \mathrm{H} & 0.08230600 & 2.91117600 & -1.85289900 \\ \mathrm{H} & -0.40212200 & 1.44804200 & -2.74712400 \\ \mathrm{H} & -1.62482400 & 2.60887700 & -2.16222500 \\ \mathrm{C} & 1.84061400 & -2.27432300 & -1.26979000 \\ \mathrm{C} & 3.49373600 & -0.76684100 & -0.31627100 \\ \mathrm{H} & 4.53203200 & -0.47833200 & -0.18008200 \\ \mathrm{H} & 3.17633000 & -1.93041900 & -1.02423800 \\ & -0.22455800 & -1.77332400 & -0.92641100 \\ \mathrm{H} & 1.60348100 & -3.17530900 & -1.82604700 \\ \mathrm{H} & & & -1.40243900\end{array}$

\section{2r-IN1a}

$\begin{array}{lrrr}\mathrm{C} & -2.86338200 & -2.39413100 & -0.24720500 \\ \mathrm{C} & -3.70759600 & -1.40627000 & -0.76777000 \\ \mathrm{C} & -3.32171200 & -0.06209900 & -0.76490700 \\ \mathrm{C} & -2.06816200 & 0.22214100 & -0.23684700 \\ \mathrm{C} & -1.19554600 & -0.74302500 & 0.27363000 \\ \mathrm{C} & -1.60792400 & -2.07244900 & 0.28584300 \\ \mathrm{~N} & -1.44657300 & 1.50875000 & -0.10332500 \\ \mathrm{C} & -0.26912700 & 1.39981300 & 0.45936600\end{array}$




$\begin{array}{lrrr}\mathrm{C} & 0.04270500 & -0.03503200 & 0.81079400 \\ \mathrm{C} & 0.76855900 & 2.44135900 & 0.60068300 \\ \mathrm{C} & 1.90296200 & 2.05189800 & -0.39071300 \\ \mathrm{C} & 0.11933000 & -0.18849700 & 2.36478800 \\ \mathrm{H} & -3.18817900 & -3.42945000 & -0.25251300 \\ \mathrm{H} & -4.67542800 & -1.68217300 & -1.17255700 \\ \mathrm{H} & -3.97892600 & 0.70624100 & -1.15689600 \\ \mathrm{H} & -0.98443300 & -2.85833100 & 0.69639600 \\ \mathrm{H} & 0.40862700 & 3.45211200 & 0.40538200 \\ \mathrm{H} & 1.16019300 & 2.41285400 & 1.62325700 \\ \mathrm{H} & 2.77814400 & 2.66896100 & -0.16648100 \\ \mathrm{H} & 1.58648600 & 2.31962100 & -1.40724400 \\ \mathrm{H} & 0.96316400 & 0.37541700 & 2.76901900 \\ \mathrm{H} & -0.80659800 & 0.14272400 & 2.84456400 \\ \mathrm{H} & 0.27236300 & -1.24258700 & 2.60528400 \\ \mathrm{C} & 2.26850300 & 0.57971000 & -0.33705000 \\ \mathrm{C} & 1.40161900 & -0.40850800 & 0.17103900 \\ \mathrm{C} & 1.80637400 & -1.75151600 & 0.15843800 \\ \mathrm{C} & 3.04517500 & -2.12434000 & -0.35686800 \\ \mathrm{C} & 3.90643900 & -1.14818200 & -0.86412300 \\ \mathrm{C} & 3.51690900 & 0.18790300 & -0.84662200 \\ \mathrm{H} & 1.15716600 & -2.51930900 & 0.56368600 \\ \mathrm{H} & 3.33804100 & -3.16929800 & -0.35488400 \\ \mathrm{H} & 4.87654600 & -1.42583700 & -1.26350600 \\ \mathrm{H} & 4.18936500 & 0.94754400 & -1.23633500 \\ \mathrm{C} & -2.10385600 & 2.72857300 & -0.58763800 \\ \mathrm{H} & -3.07110300 & 2.82842000 & -0.09068000 \\ \mathrm{H} & -1.49254300 & 3.59941500 & -0.36562600 \\ \mathrm{H} & -2.25451100 & 2.64831500 & -1.66662300\end{array}$

\section{2r-TS1b}

$\begin{array}{lrrc}\mathrm{C} & -4.36968000 & -0.55027400 & -0.39087300 \\ \mathrm{C} & -4.33605500 & 0.79447800 & 0.04213200 \\ \mathrm{C} & -3.14017400 & 1.46459800 & 0.28477800 \\ \mathrm{C} & -1.95645500 & 0.74837200 & 0.07272000 \\ \mathrm{C} & -1.97287100 & -0.60842200 & -0.33731000 \\ \mathrm{C} & -3.19526500 & -1.26337800 & -0.58056700 \\ \mathrm{~N} & -0.63882700 & 1.16443700 & 0.21382400 \\ \mathrm{C} & 0.20137000 & 0.08609900 & -0.02656100 \\ \mathrm{C} & -0.60678300 & -1.03209300 & -0.45213900 \\ \mathrm{C} & 1.66430100 & 0.24484400 & -0.21191900 \\ \mathrm{C} & 2.47081700 & -0.56912900 & 0.59560600 \\ \mathrm{C} & 1.71424500 & -1.49556300 & 1.52149700 \\ \mathrm{C} & 0.23082500 & -1.15459300 & 1.48198800\end{array}$




\section{2r-IN1b}

$\mathrm{C}$

C

C

C

C

C

$\mathrm{N}$

C

C

C

C

C

$\mathrm{H}$

$\mathrm{H}$

$\mathrm{H}$

$\mathrm{H}$

$\mathrm{H}$

$\mathrm{H}$

C

C

C

C

C

$\mathrm{N}$

C

C

C

C

C

H

H

H

H

H

H

2.23368900

$-0.12458700$

$-5.32757400$

$-5.27306700$

$-3.13670600$

$-3.21980400$

2.06827800

1.87354200

$-0.10504500$

$-0.42927300$

$-0.60647900$

$-0.39361000$

0.95827900

1.60318800

3.62289800

3.86042000

4.49507600

4.43142100

4.07561900

5.51163700

$-0.25464400$

0.83085600

$-0.62522300$

$-0.66873400$
1.12794400

$-2.25991700$

$-1.02591300$

1.32292500

2.49779000

$-2.29771300$

$-1.40309500$

$-2.54114900$

$-0.38466400$

$-2.01583900$

$-3.16285200$

$-2.17740200$

$-2.37545700$

1.74646900

1.19201900

$-0.50101600$

$-1.12475000$

0.38231600

1.87036200

0.44217400

2.42995200

2.51410900

2.48276100

3.26376600
$-1.13992000$

$-1.15548400$

$-0.57152700$

0.18767900

0.61348300

$-0.90878600$

2.55683700

1.23445200

2.17586400

1.55486400

$-0.76808500$

$-2.21504800$

$-1.09762100$

$-1.77170900$

$-1.25093700$

0.47531400

1.09825500

$-0.44399600$

$-1.96668600$

$-0.53154500$

0.83835000

0.84257800

1.86789900

0.26627700

$\begin{array}{rrc}4.32076300 & -0.62874800 & -0.04142200 \\ 4.34053100 & 0.76292200 & -0.19715100 \\ 3.15417400 & 1.50521400 & -0.21247500 \\ 1.97005000 & 0.79005500 & -0.06903500 \\ 1.92624200 & -0.59657400 & 0.09629500 \\ 3.11215600 & -1.32159100 & 0.11292100 \\ 0.62564000 & 1.29001800 & -0.07491000 \\ -0.24015400 & 0.30819400 & 0.12319600 \\ 0.48522900 & -1.01209400 & 0.27983400 \\ -1.60061300 & -2.08318700 & -0.73496300 \\ -0.07616300 & -1.92245800 & -0.84819800 \\ 0.25532000 & -1.61837300 & 1.68984100 \\ 5.25650700 & -1.17801700 & -0.03579800 \\ 5.28912700 & 1.27800600 & -0.30580100 \\ 3.17854000 & 2.58348100 & -0.32353600 \\ 3.11189900 & -2.39964300 & 0.24129500 \\ -1.99726000 & -2.47372700 & -1.67816500 \\ -1.83605400 & -2.84466000 & 0.01992700\end{array}$




$\begin{array}{lrrc}\mathrm{H} & 0.18895100 & -1.48662500 & -1.81865400 \\ \mathrm{H} & 0.41458400 & -2.89937700 & -0.79520100 \\ \mathrm{H} & -0.80269600 & -1.80678600 & 1.88407900 \\ \mathrm{H} & 0.79643500 & -2.56568800 & 1.76312800 \\ \mathrm{H} & 0.63454500 & -0.95128400 & 2.46853000 \\ \mathrm{C} & -2.43949500 & 1.48242300 & 0.50222200 \\ \mathrm{C} & -3.82528200 & 1.46231200 & 0.44048600 \\ \mathrm{C} & -4.47928800 & 0.32430200 & -0.04904100 \\ \mathrm{C} & -3.74714800 & -0.79687200 & -0.43846100 \\ \mathrm{C} & -2.35085900 & -0.81763300 & -0.36077800 \\ \mathrm{C} & -1.68530900 & 0.35757800 & 0.08934100 \\ \mathrm{H} & -1.94219100 & 2.34843100 & 0.92312100 \\ \mathrm{H} & -4.39629900 & 2.32131900 & 0.77635500 \\ \mathrm{H} & -5.56274500 & 0.30858000 & -0.11484900 \\ \mathrm{H} & -4.26855200 & -1.67693500 & -0.80429800 \\ \mathrm{C} & 0.33646100 & 2.68935800 & -0.41086300 \\ \mathrm{H} & -0.65475000 & 2.76100500 & -0.85475700 \\ \mathrm{H} & 1.07792400 & 3.02752500 & -1.13507700 \\ \mathrm{H} & 0.39287100 & 3.31530600 & 0.48357100\end{array}$

\section{2s-TS1a}

C

C

C

C

C

C

$\mathrm{N}$

C

C

C

C

C

C

C

C

$\mathrm{H}$

$\mathrm{H}$

$\mathrm{H}$

$\mathrm{H}$

$\mathrm{H}$

$\mathrm{H}$

$\mathrm{H}$

$\mathrm{H}$

$\begin{array}{rrr}3.94909000 & -2.30571500 & -0.84727900 \\ 4.27113800 & -2.13136600 & 0.51632700 \\ 3.73035800 & -1.09808400 & 1.27598700 \\ 2.85711600 & -0.21726300 & 0.62414700 \\ 2.50568200 & -0.39501100 & -0.73435400 \\ 3.05986700 & -1.44720300 & -1.48114800 \\ 2.20225300 & 0.90406100 & 1.12314300 \\ 1.36740500 & 1.41599400 & 0.13226200 \\ 1.57738500 & 0.65918700 & -1.08911800 \\ 0.80402700 & 2.82451700 & 0.17453200 \\ -0.61837300 & 2.80866700 & -0.44074900 \\ -1.08605000 & 1.37855200 & -0.34117300 \\ -0.06637200 & 0.41824500 & -0.20434800 \\ -0.35305700 & -0.92581000 & 0.07291200 \\ 1.35005500 & 1.09205300 & -2.51103000 \\ 4.39948700 & -3.12317500 & -1.39991300 \\ 4.96224400 & -2.82356700 & 0.98697900 \\ 3.98841000 & -0.98563900 & 2.32270300 \\ 2.80385700 & -1.58749400 & -2.52642200 \\ 0.75374300 & 3.16690600 & 1.20968500 \\ 1.48182600 & 3.50074500 & -0.35625300 \\ -0.61035600 & 3.15391000 & -1.48096900 \\ -1.28915300 & 3.48351000 & 0.09731700\end{array}$




$\begin{array}{crrr}\mathrm{H} & 2.28635200 & 1.51124900 & -2.89748800 \\ \mathrm{H} & 1.08471300 & 0.23468400 & -3.13450700 \\ \mathrm{H} & 0.57197900 & 1.84502900 & -2.61835700 \\ \mathrm{C} & 2.10968900 & 1.19650800 & 2.55200700 \\ \mathrm{H} & 1.77564200 & 2.22088300 & 2.70649000 \\ \mathrm{H} & 1.41694400 & 0.50915900 & 3.05114600 \\ \mathrm{H} & 3.10030800 & 1.09896000 & 2.99892000 \\ \mathrm{C} & -1.68345200 & -1.31652300 & 0.18598300 \\ \mathrm{C} & -2.41506300 & 0.96774000 & -0.23445700 \\ \mathrm{H} & -3.21805500 & 1.69276300 & -0.31569400 \\ \mathrm{C} & -2.70856100 & -0.37501200 & 0.01578400 \\ \mathrm{H} & 0.43611200 & -1.66440500 & 0.15442700 \\ \mathrm{H} & -1.92571400 & -2.35543000 & 0.38281100 \\ \mathrm{C} & -4.14674400 & -0.82877300 & 0.16481500 \\ \mathrm{~F} & -4.37378700 & -1.96584900 & -0.52881500 \\ \mathrm{~F} & -5.01923400 & 0.10280000 & -0.27065900 \\ \mathrm{~F} & -4.43575100 & -1.08481000 & 1.46286700\end{array}$

\section{2s-TS1b}

\begin{tabular}{|c|c|c|c|}
\hline $\mathrm{C}$ & -5.60617700 & -0.35811600 & -0.57193200 \\
\hline $\mathrm{C}$ & -5.52739700 & 0.97198400 & -0.10136300 \\
\hline $\mathrm{C}$ & -4.31337800 & 1.57354500 & 0.21935900 \\
\hline $\mathrm{C}$ & -3.15841500 & 0.80277700 & 0.04661500 \\
\hline $\mathrm{C}$ & -3.22155100 & -0.54087800 & -0.39995500 \\
\hline $\mathrm{C}$ & -4.46102700 & -1.12613900 & -0.72191700 \\
\hline $\mathrm{N}$ & -1.82889000 & 1.14783400 & 0.26251200 \\
\hline $\mathrm{C}$ & -1.03561100 & 0.03465600 & 0.03493800 \\
\hline $\mathrm{C}$ & -1.87434900 & -1.03192000 & -0.45901800 \\
\hline $\mathrm{C}$ & 0.44142700 & 0.12026100 & -0.07285000 \\
\hline $\mathrm{C}$ & 1.16184700 & -0.75145900 & 0.75250900 \\
\hline $\mathrm{C}$ & 0.31594000 & -1.66157400 & 1.61481900 \\
\hline $\mathrm{C}$ & -1.14709500 & -1.25168800 & 1.50626800 \\
\hline $\mathrm{C}$ & 1.10349800 & 0.99071900 & -0.94974200 \\
\hline $\mathrm{C}$ & -1.41918800 & -2.26218400 & -1.17664700 \\
\hline $\mathrm{H}$ & -6.57615400 & -0.77911700 & -0.81333300 \\
\hline $\mathrm{H}$ & -6.44283500 & 1.54444900 & 0.01101500 \\
\hline $\mathrm{H}$ & -4.27539900 & 2.59692000 & 0.57541900 \\
\hline $\mathrm{H}$ & -4.52071900 & -2.14946600 & -1.07874900 \\
\hline $\mathrm{H}$ & 0.61991800 & -1.60936300 & 2.66854600 \\
\hline $\mathrm{H}$ & 0.44461200 & -2.70592500 & 1.30891600 \\
\hline $\mathrm{H}$ & -1.48695800 & -0.48740200 & 2.20447000 \\
\hline $\mathrm{H}$ & -1.84625200 & -2.08462200 & 1.51874500 \\
\hline $\mathrm{H}$ & -1.97603700 & -3.14688000 & -0.85345200 \\
\hline $\mathrm{H}$ & -1.61436100 & -2.12835800 & -2.24712000 \\
\hline
\end{tabular}


$\mathrm{H}$

$\mathrm{H}$

$\mathrm{C}$

C

$\mathrm{H}$

$\mathrm{C}$

C

$\mathrm{H}$

$\mathrm{H}$

$\mathrm{H}$

$\mathrm{H}$

C

F

F

F

\section{2t-TS1a}

$\mathrm{C}$

C

C

C

C

C

$\mathrm{N}$

C

C

C

C

C

C

C

C

$\mathrm{H}$

$\mathrm{H}$

$\mathrm{H}$

$\mathrm{H}$

$\mathrm{H}$

$\mathrm{H}$

$\mathrm{H}$

$\mathrm{H}$

$\mathrm{H}$

$\mathrm{H}$

$\mathrm{H}$

C

\begin{tabular}{rrr}
-0.35098400 & -2.44616500 & \multicolumn{1}{c}{-1.05654600} \\
0.54253000 & 1.65501700 & -1.59967300 \\
2.49529500 & 0.98545200 & -0.99146300 \\
2.55727700 & -0.75588000 & 0.70701100 \\
3.12898800 & -1.42551300 & 1.34080200 \\
3.21611800 & 0.11541900 & -0.16211800 \\
-1.41490100 & 2.37599500 & 0.94192200 \\
-0.32794000 & 2.41159400 & 0.99424100 \\
-1.82620000 & 2.41562100 & 1.95633300 \\
-1.76717000 & 3.24344000 & 0.37878600 \\
3.02080500 & 1.64935900 & -1.66937900 \\
4.72926300 & 0.16204900 & -0.19583200 \\
5.19152800 & 0.19807200 & -1.46558200 \\
5.28333500 & -0.90762500 & 0.41345500 \\
5.19257500 & 1.27104800 & 0.42987900
\end{tabular}

3.34216200

1.85169200

1.06103900

3.67994700

1.74397900

$-0.30541900$

3.07423700

0.81241400

$-1.14384800$

2.11749300

$-0.03481900$

$-0.56975400$

1.75122900

0.07859300

0.79149800

2.37201800

1.02829400

1.61856900

1.38303200

$-1.05917700$

$-1.15837400$

0.48266500

$-1.56350800$

$-0.22057800$

0.73010400

$-0.91552100$

1.05553800

$-0.18856800$

$-2.91700100$

$-0.37051900$

$-1.61680800$

$-2.82942700$

0.22442700

$-1.96554300$

$-1.36232100$

0.22027100

$-0.86694500$

$-0.48295100$

0.16350500

$-1.02701600$

0.89640800

$-0.02509100$

0.44075800

$-1.42606500$

2.44004400

3.84343600

2.59099800

1.67635800

4.43468900

2.40765200

$-0.71550000$

3.34510600

0.74988900

$-2.19154500$

2.10353100

1.11930000

2.66615600

$-0.24542900$

$-3.17990000$

$-1.42833100$

0.42441700

$-3.68050800$

0.11915700

$-1.65563600$

$-3.24780500$

1.23692700

$-2.32823200$

$-3.40848800$

$-0.37031300$

$1.33399800-1.94204300$

2.81113400

0.22920300

$-0.59496200$

3.11742900

$-0.39463700$

$-2.12184100$

2.48322700

1.29166700

$-1.22932400$ 


$\begin{array}{lrrr}\mathrm{H} & 0.82640000 & -2.18382800 & -2.84542900 \\ \mathrm{H} & 0.71019900 & -0.41922500 & -3.06194600 \\ \mathrm{H} & 2.29791500 & -1.23365500 & -3.02939900 \\ \mathrm{C} & -2.32313100 & 1.39691400 & -0.13268800 \\ \mathrm{C} & -3.24989400 & -0.82570200 & 0.12137300 \\ \mathrm{H} & -4.11428700 & -1.48317200 & 0.13651300 \\ \mathrm{C} & -3.43473400 & 0.54856100 & -0.04263400 \\ \mathrm{H} & -0.18165100 & 1.57365900 & -0.04233900 \\ \mathrm{H} & -4.43389100 & 0.96321400 & -0.11850800 \\ \mathrm{Cl} & -2.56083600 & 3.11080300 & -0.35348700\end{array}$

\section{2t-TS1b}

C

C

C

C

C

C

$\mathrm{N}$

C

C

C

C

C

C

C

C

$\mathrm{H}$

$\mathrm{H}$

$\mathrm{H}$

$\mathrm{H}$

$\mathrm{H}$

$\mathrm{H}$

$\mathrm{H}$

$\mathrm{H}$

$\mathrm{H}$

$\mathrm{H}$

$\mathrm{H}$

$\mathrm{H}$

C

C

$\mathrm{H}$

C

C

$\begin{array}{rcc}4.82711400 & -0.29591200 & -0.80526100 \\ 4.64227800 & -1.37422000 & 0.08921200 \\ 3.40413800 & -1.66106800 & 0.65755600 \\ 2.33363400 & -0.83529100 & 0.29654400 \\ 2.50663300 & 0.26501800 & -0.58007300 \\ 3.76821200 & 0.53301800 & -1.14483600 \\ 1.00126300 & -0.90039600 & 0.68650700 \\ 0.32071900 & 0.17517900 & 0.13669200 \\ 1.22449200 & 0.89124700 & -0.73199200 \\ -1.15980500 & 0.26543300 & 0.14143400 \\ -1.69601400 & 1.45236600 & 0.65616500 \\ -0.67452500 & 2.46883600 & 1.11420700 \\ 0.71463000 & 1.84494200 & 1.07967000 \\ -1.98569100 & -0.75296300 & -0.35046500 \\ 0.84566100 & 1.86849800 & -1.79807600 \\ 5.81111200 & -0.11725200 & -1.22505800 \\ 5.49266200 & -2.00091500 & 0.33921200 \\ 3.28342700 & -2.49559000 & 1.33912600 \\ 3.90986400 & 1.36430900 & -1.82825700 \\ -0.88014900 & 2.81011800 & 2.13752000 \\ -0.70831000 & 3.36182200 & 0.47968100 \\ 1.02591300 & 1.31229100 & 1.97772600 \\ 1.50479700 & 2.52737700 & 0.77509000 \\ 1.52866000 & 2.72305600 & -1.82328900 \\ 0.91942100 & 1.36432200 & -2.76880800 \\ -0.17798100 & 2.22892600 & -1.69042800 \\ -1.57095400 & -1.66689300 & -0.76194800 \\ -3.36748100 & -0.56360600 & -0.31429200 \\ -3.08224000 & 1.62310900 & 0.67910500 \\ -3.51712300 & 2.53511900 & 1.07745300 \\ -3.92000300 & 0.61580300 & 0.19941300 \\ 0.51608900 & -1.76948900 & 1.75888800\end{array}$


$\mathrm{H}$

$\mathrm{H}$

$\mathrm{H}$

$\mathrm{H}$

$\mathrm{Cl}$

\section{2u-TS1a}

$\mathrm{C}$

C

C

C

C

C

$\mathrm{N}$

C

C

C

C

C

C

C

C

$\mathrm{H}$

$\mathrm{H}$

$\mathrm{H}$

$\mathrm{H}$

$\mathrm{H}$

$\mathrm{H}$

$\mathrm{H}$

$\mathrm{H}$

$\mathrm{H}$

$\mathrm{H}$

$\mathrm{H}$

C

$\mathrm{H}$

$\mathrm{H}$

$\mathrm{H}$

C

C

$\mathrm{H}$

C

$\mathrm{H}$

$\mathrm{H}$

C

$\begin{array}{ccc}-0.55543900 & -1.62329800 & 1.88475800 \\ 1.02488300 & -1.54195000 & 2.70180900 \\ 0.69829200 & -2.81442800 & 1.49663700 \\ -4.99718900 & 0.73859300 & 0.22347300 \\ -4.41810500 & -1.81813200 & -0.92021100\end{array}$

$\begin{array}{rcc}3.54856400 & 1.36273000 & 0.99793200 \\ 3.87365100 & 1.09608400 & -0.34914000 \\ 3.09024900 & 0.26602000 & -1.14616600 \\ 1.96322600 & -0.31508600 & -0.54960500 \\ 1.61072000 & -0.03456200 & 0.79057400 \\ 2.41282800 & 0.80781200 & 1.57563600 \\ 1.03611000 & -1.19783300 & -1.09275900 \\ 0.03065300 & -1.42983700 & -0.15057300 \\ 0.39320700 & -0.76626500 & 1.08867500 \\ -0.90479800 & -2.62341700 & -0.23017600 \\ -2.28768300 & -2.19841600 & 0.32415300 \\ -2.31363100 & -0.69399600 & 0.22406400 \\ -1.05283900 & -0.07342800 & 0.12926400 \\ -0.92305100 & 1.29625100 & -0.15361100 \\ -0.00864700 & -1.11457700 & 2.49544900 \\ 4.19171800 & 2.01165400 & 1.58259700 \\ 4.76172500 & 1.55055800 & -0.77713600 \\ 3.35681800 & 0.07736200 & -2.17977800 \\ 2.15618300 & 1.02167600 & 2.60827700 \\ -1.00486700 & -2.93881500 & -1.27048000 \\ -0.46942800 & -3.46306000 & 0.32116800 \\ -2.42613300 & -2.53498400 & 1.35830300 \\ -3.10027000 & -2.64889800 & -0.25213000 \\ 0.74917900 & -1.78560700 & 2.91635000 \\ -0.04188200 & -0.21575700 & 3.11633600 \\ -0.97542200 & -1.60918100 & 2.56285700 \\ 0.91858000 & -1.43529900 & -2.52882400 \\ 0.31124400 & -2.31930000 & -2.71592700 \\ 0.47071800 & -0.57315600 & -3.03743700 \\ 1.91163800 & -1.62107600 & -2.94110600 \\ -2.06580800 & 2.08301300 & -0.32052400 \\ -3.45050700 & 0.09842200 & 0.06505000 \\ -4.43598500 & -0.35707900 & 0.10412100 \\ -3.32491800 & 1.46484300 & -0.19023900 \\ 0.05627500 & 1.76075800 & -0.19657000 \\ -4.22292700 & 2.06520300 & -0.30849200 \\ -1.96229600 & 3.56216500 & -0.60557200\end{array}$


$\mathrm{H}$

$\mathrm{H}$

$\mathrm{H}$

\section{2u-TS1b}

C

C

C

C

C

C

$\mathrm{N}$

C

C

C

C

C

C

C

C

$\mathrm{H}$

$\mathrm{H}$

$\mathrm{H}$

$\mathrm{H}$

$\mathrm{H}$

$\mathrm{H}$

$\mathrm{H}$

$\mathrm{H}$

$\mathrm{H}$

$\mathrm{H}$

$\mathrm{H}$

$\mathrm{H}$

C

C

$\mathrm{H}$

C

C

$\mathrm{H}$

$\mathrm{H}$

$\mathrm{H}$

$\mathrm{H}$

C

$\mathrm{H}$

$\mathrm{H}$
$-0.92193600$

$-2.48588700$

$-2.41855000$

4.62011300

4.49625000

3.26768100

2.14354000

2.25344300

3.50707000

0.81002500

0.05771600

0.92892400

$-1.42421200$

$-2.07475300$

$-1.15948000$

0.27397200

$-2.14997100$

0.50612300

5.59955700

5.38763600

3.19401300

3.60192700

$-1.43173900$

$-1.23688500$

0.58468200

1.02228700

1.10225300

0.67372700

$-0.55208000$

$-1.62689200$

$-3.54841400$

$-3.47088900$

$-3.99720500$

$-4.19071500$

0.36228500

$-0.72209500$

0.81215300

0.64499300

$-5.27646600$

$-4.35447000$

$-3.71215700$

$-4.95497200$
3.88061300

$-0.70619600$

3.81972400

$-1.53234200$

4.14944300

0.19870500

0.07611000

$-0.69347500$

$-1.11702800$

0.05368500

$-1.57064300$

0.52563900

$-0.79463700$

0.21962200

0.41689800

$-0.50821800$

0.85337900

$-0.97746300$

$-1.01357900$

0.53867900

0.06588300

0.09480700

0.95296200

$-0.63894300$

0.03411000

0.04360200

1.09994300

0.67440400

2.13138300

1.29490900

1.62036900

1.25798400

$-0.97577500$

$-0.60490800$

2.02830400

$-1.58709500$

0.38422100

$-1.04305500$

$-1.69963000$

0.26450600

$-2.48987100$

1.09575800

1.77219400

$-1.54750400$

2.33572200

2.33908600

3.08720300

0.76411000

0.99933300

2.09750500

2.38999300

1.08340200

2.93718800

$-1.45977400$

1.67063800

$-2.60976100$

2.27404400

$-1.49198200$

$-1.78912200$

$-1.10066900$

$-0.93266200$

$-0.62858100$

1.15057600

0.65410600

1.96653900

1.14134200

0.14257300

0.01435900

$-2.04207800$

1.47676100

$-1.99928500$

1.56515100

$-1.88935400$

2.46393700

$-3.02965600$

1.10418200

0.18696900

0.01265100

$-2.00215900$

$-1.32563900$

$-2.77456600$

$-1.75603100$

$-1.57468100$

$-2.13597800$ 


\section{2v-TS1a}

$\mathrm{C}$

$\mathrm{C}$

$\mathrm{C}$

C

$\mathrm{C}$

$\mathrm{C}$

$\mathrm{N}$

C

$\mathrm{C}$

$\mathrm{C}$

$\mathrm{C}$

$\mathrm{C}$

$\mathrm{C}$

$\mathrm{C}$

C

$\mathrm{H}$

$\mathrm{H}$

$\mathrm{H}$

$\mathrm{H}$

$\mathrm{H}$

$\mathrm{H}$

$\mathrm{H}$

$\mathrm{H}$

$\mathrm{H}$

$\mathrm{H}$

$\mathrm{H}$

$\mathrm{C}$

$\mathrm{H}$

$\mathrm{H}$

$\mathrm{H}$

C

C

$\mathrm{H}$

C

$\mathrm{H}$

$\mathrm{H}$

$\mathrm{F}$

\section{2v-TS1b}

$\mathrm{C}$

C

$\begin{array}{rcc}-3.62437300 & -1.71976800 & 0.75405600 \\ -3.96587100 & -1.28046900 & -0.54275400 \\ -3.21974300 & -0.31634800 & -1.21520800 \\ -2.11362600 & 0.21973000 & -0.54254500 \\ -1.74480900 & -0.23082800 & 0.74605600 \\ -2.50918800 & -1.20584500 & 1.40510500 \\ -1.22333400 & 1.20440500 & -0.95805400 \\ -0.22036200 & 1.33927600 & 0.00625400 \\ -0.55518500 & 0.49977500 & 1.14587900 \\ 0.66859700 & 2.56830300 & 0.09479800 \\ 2.06229400 & 2.12535200 & 0.60620900 \\ 2.14970100 & 0.65073600 & 0.30066000 \\ 0.90414600 & 0.00851800 & 0.10806800 \\ 0.83360900 & -1.31414300 & -0.36553700 \\ -0.17246200 & 0.67443600 & 2.59022500 \\ -4.23799300 & -2.46991300 & 1.24114200 \\ -4.83681900 & -1.70603300 & -1.03130000 \\ -3.49804500 & 0.00366000 & -2.21270700 \\ -2.23966500 & -1.55150700 & 2.39795700 \\ 0.76617000 & 3.01934500 & -0.89459800 \\ 0.19891500 & 3.31300400 & 0.74508400 \\ 2.17794300 & 2.31711700 & 1.67908400 \\ 2.86304000 & 2.68038600 & 0.11054900 \\ -0.95789600 & 1.25469100 & 3.08823500 \\ -0.10671400 & -0.29606800 & 3.08844600 \\ 0.77319100 & 1.19355100 & 2.73108200 \\ -1.12667400 & 1.64611800 & -2.34681100 \\ -0.54778400 & 2.56586400 & -2.41040000 \\ -0.65947700 & 0.87924800 & -2.97626700 \\ -2.12884300 & 1.85859400 & -2.72254600 \\ 2.00904300 & -2.01031400 & -0.61891000 \\ 3.32483600 & -0.05199900 & 0.05513900 \\ 4.30107300 & 0.41076600 & 0.15700800 \\ 3.22902700 & -1.37093700 & -0.38691000 \\ -0.12135800 & -1.81183200 & -0.49052400 \\ 1.99748800 & -3.03688300 & -0.96749100 \\ 4.36070600 & -2.04937500 & -0.62100200\end{array}$

$-4.69847800$

$\begin{array}{ll}-0.47480800 & -0.46559800\end{array}$

$0.86460700 \quad-0.01812200$ 


\begin{tabular}{|c|c|c|c|}
\hline $\mathrm{C}$ & -3.44255400 & 1.50759600 & 0.25644100 \\
\hline $\mathrm{C}$ & -2.26973200 & 0.76917700 & 0.06188100 \\
\hline $\mathrm{C}$ & -2.30510100 & -0.58282000 & -0.36246600 \\
\hline $\mathrm{C}$ & -3.53520600 & -1.21012600 & -0.63806800 \\
\hline $\mathrm{N}$ & -0.94670100 & 1.15675200 & 0.23526200 \\
\hline $\mathrm{C}$ & -0.12411200 & 0.06401700 & 0.00095800 \\
\hline $\mathrm{C}$ & -0.94603800 & -1.03315700 & -0.45416800 \\
\hline $\mathrm{C}$ & 1.34476200 & 0.19451300 & -0.14906700 \\
\hline $\mathrm{C}$ & 2.11694700 & -0.64566200 & 0.66646900 \\
\hline $\mathrm{C}$ & 1.32206100 & -1.56742200 & 1.56350100 \\
\hline $\mathrm{C}$ & -0.15319400 & -1.19571600 & 1.49448900 \\
\hline $\mathrm{C}$ & 1.95714100 & 1.07522700 & -1.05313100 \\
\hline $\mathrm{C}$ & -0.47441600 & -2.26222500 & -1.16256400 \\
\hline $\mathrm{H}$ & -5.66185400 & -0.92868600 & -0.67126600 \\
\hline $\mathrm{H}$ & -5.57536900 & 1.41086100 & 0.11314500 \\
\hline $\mathrm{H}$ & -3.42520800 & 2.53728800 & 0.59552000 \\
\hline $\mathrm{H}$ & -3.57397300 & -2.24039800 & -0.97746400 \\
\hline $\mathrm{H}$ & 1.65635700 & -1.49541200 & 2.60687000 \\
\hline $\mathrm{H}$ & 1.46846800 & -2.61151000 & 1.26477700 \\
\hline $\mathrm{H}$ & -0.48886100 & -0.42791800 & 2.19079300 \\
\hline $\mathrm{H}$ & -0.83035200 & -2.04530000 & 1.54398300 \\
\hline $\mathrm{H}$ & -0.98212700 & -3.15976300 & -0.79624800 \\
\hline $\mathrm{H}$ & -0.72002300 & -2.16185700 & -2.22627000 \\
\hline $\mathrm{H}$ & 0.60443200 & -2.40119400 & -1.08445400 \\
\hline $\mathrm{H}$ & 1.35795400 & 1.71567500 & -1.69297300 \\
\hline $\mathrm{C}$ & 3.34689100 & 1.11733100 & -1.13920600 \\
\hline $\mathrm{C}$ & 3.50915000 & -0.61339100 & 0.58499600 \\
\hline $\mathrm{H}$ & 4.13970900 & -1.24478100 & 1.20234900 \\
\hline $\mathrm{C}$ & 4.09130500 & 0.27281400 & -0.31599800 \\
\hline $\mathrm{H}$ & 3.85558800 & 1.78323900 & -1.82704400 \\
\hline $\mathrm{C}$ & -0.55184900 & 2.40720700 & 0.88355600 \\
\hline $\mathrm{H}$ & 0.53468700 & 2.46824900 & 0.91698900 \\
\hline $\mathrm{H}$ & -0.94744100 & 2.45674700 & 1.90384100 \\
\hline $\mathrm{H}$ & -0.93361000 & 3.25574300 & 0.31066300 \\
\hline $\mathrm{F}$ & 5.43356500 & 0.31875400 & -0.39371800 \\
\hline
\end{tabular}

\section{2w-TS1a}

C

C

C

C

C

C

$\mathrm{N}$

$\begin{array}{ccc}-3.54559500 & -2.14892000 & 0.91971200 \\ -3.90747000 & -1.95174300 & -0.42849200 \\ -3.32711600 & -0.95441500 & -1.20878700 \\ -2.37098700 & -0.13367700 & -0.59585900 \\ -1.97857800 & -0.34035600 & 0.74660100 \\ -2.57418900 & -1.35062600 & 1.51469100 \\ -1.67304900 & 0.94862200 & -1.12205400\end{array}$




\begin{tabular}{|c|c|c|c|}
\hline $\mathrm{C}$ & -0.73984400 & 1.38731900 & -0.16598500 \\
\hline $\mathrm{C}$ & -0.96325800 & 0.65221600 & 1.07343900 \\
\hline $\mathrm{C}$ & -0.13125600 & 2.78100700 & -0.21345200 \\
\hline $\mathrm{C}$ & 1.28684400 & 2.70062400 & 0.40575500 \\
\hline $\mathrm{C}$ & 1.67774000 & 1.24565200 & 0.30694100 \\
\hline $\mathrm{C}$ & 0.57603900 & 0.35986000 & 0.13780000 \\
\hline $\mathrm{C}$ & 0.80594900 & -0.99457000 & -0.17483900 \\
\hline $\mathrm{C}$ & -0.71562400 & 1.08775800 & 2.49278800 \\
\hline $\mathrm{H}$ & -4.02723300 & -2.93397900 & 1.49268400 \\
\hline $\mathrm{H}$ & -4.66175100 & -2.59404600 & -0.87227700 \\
\hline $\mathrm{H}$ & -3.62023000 & -0.82185600 & -2.24394100 \\
\hline $\mathrm{H}$ & -2.28647800 & -1.50934300 & 2.54921900 \\
\hline $\mathrm{H}$ & -0.06252400 & 3.11130400 & -1.25222800 \\
\hline $\mathrm{H}$ & -0.78240400 & 3.48829100 & 0.30943700 \\
\hline $\mathrm{H}$ & 1.29088300 & 3.04590500 & 1.44569300 \\
\hline $\mathrm{H}$ & 1.99526300 & 3.33824200 & -0.12945700 \\
\hline $\mathrm{H}$ & -1.62560000 & 1.56496500 & 2.87461600 \\
\hline $\mathrm{H}$ & -0.50186800 & 0.22207400 & 3.12494900 \\
\hline $\mathrm{H}$ & 0.10666600 & 1.79260100 & 2.59642100 \\
\hline $\mathrm{C}$ & -1.55787100 & 1.19242300 & -2.55569000 \\
\hline $\mathrm{H}$ & -1.21423500 & 2.20993300 & -2.73790100 \\
\hline $\mathrm{H}$ & -0.86382200 & 0.48558400 & -3.02828900 \\
\hline $\mathrm{H}$ & -2.54183300 & 1.08998000 & -3.01560700 \\
\hline $\mathrm{C}$ & 2.10279000 & -1.47498200 & -0.27934400 \\
\hline $\mathrm{C}$ & 2.96706900 & 0.76459700 & 0.20548100 \\
\hline $\mathrm{H}$ & 3.82572600 & 1.42393100 & 0.28380800 \\
\hline C & 3.19425300 & -0.60281700 & -0.06989700 \\
\hline $\mathrm{H}$ & -0.02405900 & -1.68393600 & -0.28464600 \\
\hline $\mathrm{H}$ & 2.26351300 & -2.52327400 & -0.49704800 \\
\hline $\mathrm{O}$ & 4.47992100 & -0.96934700 & -0.13375900 \\
\hline $\mathrm{C}$ & 4.83102700 & -2.32498900 & -0.45796400 \\
\hline $\mathrm{H}$ & 4.45333000 & -2.59620900 & -1.44875300 \\
\hline $\mathrm{H}$ & 5.91951500 & -2.34611600 & -0.46005600 \\
\hline $\mathrm{H}$ & 4.44956200 & -3.01578800 & 0.30041500 \\
\hline \multicolumn{4}{|c|}{ 2w-TS1b } \\
\hline $\mathrm{C}$ & 5.04486300 & 0.26239900 & -0.64276000 \\
\hline $\mathrm{C}$ & 4.95043400 & -1.04019200 & -0.10268500 \\
\hline $\mathrm{C}$ & 3.73162000 & -1.60207800 & 0.26690100 \\
\hline $\mathrm{C}$ & 2.58604900 & -0.82121000 & 0.07229600 \\
\hline $\mathrm{C}$ & 2.66583500 & 0.49634300 & -0.44566500 \\
\hline $\mathrm{C}$ & 3.91044800 & 1.04144500 & -0.81501400 \\
\hline $\mathrm{N}$ & 1.25725800 & -1.12934600 & 0.32741500 \\
\hline $\mathrm{C}$ & 0.47319600 & -0.01449600 & 0.05102200 \\
\hline $\mathrm{C}$ & 1.32681400 & 1.00524500 & -0.51274000 \\
\hline
\end{tabular}




$\begin{array}{lrrc}\mathrm{C} & -1.00235600 & -0.08534500 & -0.03031800 \\ \mathrm{C} & -1.70701200 & 0.84640600 & 0.75178400 \\ \mathrm{C} & -0.83080400 & 1.78922500 & 1.54777300 \\ \mathrm{C} & 0.61806900 & 1.33120300 & 1.45820300 \\ \mathrm{C} & -1.70195700 & -0.99068600 & -0.83600100 \\ \mathrm{C} & 0.88033600 & 2.20794500 & -1.27936400 \\ \mathrm{H} & 6.01796600 & 0.65303400 & -0.92006900 \\ \mathrm{H} & 5.85760500 & -1.62240300 & 0.02601600 \\ \mathrm{H} & 3.68144300 & -2.60485900 & 0.67621000 \\ \mathrm{H} & 3.98164800 & 2.04413900 & -1.22461300 \\ \mathrm{H} & -1.12723000 & 1.81439700 & 2.60473500 \\ \mathrm{H} & -0.93178900 & 2.81468300 & 1.17486100 \\ \mathrm{H} & 0.93321100 & 0.58931500 & 2.19125300 \\ \mathrm{H} & 1.34528500 & 2.13931800 & 1.42896900 \\ \mathrm{H} & 1.44089100 & 3.10312900 & -0.99278800 \\ \mathrm{H} & 1.07578900 & 2.02926100 & -2.34324700 \\ \mathrm{H} & -0.18745300 & 2.39894600 & -1.16727400 \\ \mathrm{H} & -1.16712600 & -1.70600400 & -1.45405400 \\ \mathrm{C} & -3.09607100 & -0.97147000 & -0.86372300 \\ \mathrm{C} & -3.09344100 & 0.87429200 & 0.72983100 \\ \mathrm{H} & -3.65978000 & 1.57977800 & 1.32963500 \\ \mathrm{C} & -3.79832800 & -0.03856700 & -0.07753600 \\ \mathrm{H} & -3.62310000 & -1.67564500 & -1.49507000 \\ \mathrm{C} & 0.83186400 & -2.31177000 & 1.07434000 \\ \mathrm{H} & -0.25385500 & -2.31132400 & 1.15685000 \\ \mathrm{H} & 1.27075100 & -2.31534800 & 2.07815000 \\ \mathrm{H} & 1.14368000 & -3.21571300 & 0.54522700 \\ \mathrm{O} & -5.14285200 & 0.05878800 & -0.02492000 \\ \mathrm{C} & -5.94782000 & -0.83477000 & -0.80309600 \\ \mathrm{H} & -5.75447300 & -0.70535400 & -1.87351000 \\ \mathrm{H} & -6.97902900 & -0.56198300 & -0.58300000 \\ \mathrm{H} & -5.77286100 & -1.87575200 & -0.51032100\end{array}$

\section{2x-TS1a}

$\mathrm{C}$
$\mathrm{C}$
$\mathrm{C}$
$\mathrm{C}$
$\mathrm{C}$
$\mathrm{C}$
$\mathrm{N}$
$\mathrm{C}$
$\mathrm{C}$
$\mathrm{C}$

$\begin{array}{ccc}3.43676700 & -2.20622500 & -1.03154200 \\ 3.79112300 & -2.11554100 & 0.33024000 \\ 3.27262600 & -1.12645400 & 1.16260300 \\ 2.38777300 & -0.20423100 & 0.58828300 \\ 2.00284600 & -0.30123800 & -0.76873400 \\ 2.53521700 & -1.30589500 & -1.58917800 \\ 1.75711100 & 0.88979600 & 1.17121000 \\ 0.87707800 & 1.45359900 & 0.23323700 \\ 1.06797700 & 0.78002100 & -1.04594900 \\ 0.36711000 & 2.87994900 & 0.35249500\end{array}$




$\begin{array}{lrrc}\mathrm{C} & -1.04058000 & 2.93000000 & -0.29134100 \\ \mathrm{C} & -1.53328900 & 1.50302700 & -0.27969300 \\ \mathrm{C} & -0.50935100 & 0.53155800 & -0.14922400 \\ \mathrm{C} & -0.80265900 & -0.83996100 & 0.09765700 \\ \mathrm{C} & 0.87646700 & 1.31448400 & -2.43974200 \\ \mathrm{H} & 3.86887300 & -2.99004300 & -1.64425600 \\ \mathrm{H} & 4.48941200 & -2.83694300 & 0.74304700 \\ \mathrm{H} & 3.55653900 & -1.07735700 & 2.20762100 \\ \mathrm{H} & 2.25245300 & -1.38251800 & -2.63429100 \\ \mathrm{H} & 0.30008100 & 3.15673400 & 1.40683500 \\ \mathrm{H} & 1.07505100 & 3.56818200 & -0.11999100 \\ \mathrm{H} & -1.00504500 & 3.33611500 & -1.30892400 \\ \mathrm{H} & -1.71328800 & 3.58288200 & 0.27143100 \\ \mathrm{H} & 1.82747600 & 1.73666900 & -2.78457800 \\ \mathrm{H} & 0.60360600 & 0.50569700 & -3.12262300 \\ \mathrm{H} & 0.11532700 & 2.08839300 & -2.51056500 \\ \mathrm{C} & 1.65734700 & 1.06618100 & 2.61628500 \\ \mathrm{H} & 1.33435600 & 2.07982000 & 2.84910700 \\ \mathrm{H} & 0.95258700 & 0.35111800 & 3.05880700 \\ \mathrm{H} & 2.64258200 & 0.92208800 & 3.06289900 \\ \mathrm{C} & -2.13339600 & -1.16900300 & 0.15780300 \\ \mathrm{C} & -2.88110400 & 1.13179300 & -0.22067000 \\ \mathrm{H} & -3.68031900 & 1.86213400 & -0.28437700 \\ \mathrm{C} & -3.15091800 & -0.21366600 & -0.01803000 \\ \mathrm{H} & -0.03134000 & -1.59604900 & 0.17472600 \\ \mathrm{O} & -4.35723900 & -0.80971800 & 0.06153900 \\ \mathrm{C} & -4.11892000 & -2.20953100 & 0.34608500 \\ \mathrm{H} & -4.52222100 & -2.44531100 & 1.33472500 \\ \mathrm{H} & -4.57306800 & -2.81512600 & -0.44095500 \\ \mathrm{O} & -2.69345200 & -2.39881500 & 0.34338300 \\ & & & \\ & & & \\ \mathrm{H} & & \end{array}$

\section{2x-TS1b}

$\mathrm{C}$

$5.12737400 \quad-0.00936900$

$-0.77175600$

$4.98965600 \quad-1.21202100$

$-0.04269200$

C

3.76011200

$-1.64404100$

0.44688500

C

2.64937000

$-0.83571600$

0.17802200

$\mathrm{C}$

2.77401000

0.38509700

$-0.53152600$

C

4.02795600

0.79932300

$-1.01928000$

1.31816500

$-1.02696000$

0.52333900

C

0.58233000

0.07813300

0.11326800

C

1.46020500

0.95460600

$-0.62629900$

$-0.89858800$

0.07979100

0.09693200

C

$-1.51113800$

1.15168300

0.75514700

$\mathrm{C}$

$-0.55490200$

2.15150600

1.36583700 


$\begin{array}{lrrc}\mathrm{C} & 0.86418600 & 1.60671500 & 1.28870300 \\ \mathrm{C} & -1.64910100 & -0.92585700 & -0.55902400 \\ \mathrm{C} & 1.04347900 & 2.05248700 & -1.55082400 \\ \mathrm{H} & 6.10667000 & 0.28110100 & -1.13653600 \\ \mathrm{H} & 5.87060200 & -1.81959400 & 0.13958500 \\ \mathrm{H} & 3.67540200 & -2.57134700 & 1.00223300 \\ \mathrm{H} & 4.13359900 & 1.72536100 & -1.57559100 \\ \mathrm{H} & -0.79759100 & 2.34923200 & 2.41875900 \\ \mathrm{H} & -0.62095900 & 3.11600600 & 0.84899400 \\ \mathrm{H} & 1.17848000 & 0.96723800 & 2.11286900 \\ \mathrm{H} & 1.62548400 & 2.36286300 & 1.11062900 \\ \mathrm{H} & 1.65897300 & 2.94790700 & -1.42004400 \\ \mathrm{H} & 1.18729400 & 1.70731400 & -2.58135000 \\ \mathrm{H} & -0.00808400 & 2.31647700 & -1.43333900 \\ \mathrm{H} & -1.18134700 & -1.75430600 & -1.07894000 \\ \mathrm{C} & -3.01948700 & -0.78442200 & -0.51334100 \\ \mathrm{C} & -2.91277000 & 1.27672300 & 0.78768300 \\ \mathrm{H} & -3.40739700 & 2.09623400 & 1.29757200 \\ \mathrm{C} & -3.63570100 & 0.28985000 & 0.14497400 \\ \mathrm{C} & 0.86722600 & -2.05686900 & 1.45825100 \\ \mathrm{H} & -0.21061900 & -1.97502700 & 1.58865400 \\ \mathrm{H} & 1.35882600 & -1.93962600 & 2.43021800 \\ \mathrm{H} & 1.09820000 & -3.04725900 & 1.05820700 \\ \mathrm{O} & -4.98643100 & 0.16818700 & 0.05239600 \\ \mathrm{C} & -5.24207400 & -0.97953300 & -0.78316600 \\ \mathrm{H} & -5.68201800 & -0.64768400 & -1.72886300 \\ \mathrm{H} & -5.89134600 & -1.67719000 & -0.25047900 \\ \mathrm{O} & -3.97034000 & -1.60787500 & -1.03898600\end{array}$

\section{2y-TS1a}

$\begin{array}{lrrc}\mathrm{C} & -3.41491300 & 1.52385600 & -1.36813200 \\ \mathrm{C} & -3.88459200 & 1.44705700 & -0.04117000 \\ \mathrm{C} & -3.29036700 & 0.61170300 & 0.90348200 \\ \mathrm{C} & -2.21060200 & -0.16825300 & 0.47348700 \\ \mathrm{C} & -1.71058500 & -0.08175400 & -0.84543600 \\ \mathrm{C} & -2.32185600 & 0.76687200 & -1.77837200 \\ \mathrm{~N} & -1.44940100 & -1.07957800 & 1.19071100 \\ \mathrm{C} & -0.45525100 & -1.60759800 & 0.36210900 \\ \mathrm{C} & -0.61169000 & -1.03263700 & -0.97248100 \\ \mathrm{C} & 0.12790300 & -2.98327700 & 0.60409600 \\ \mathrm{C} & 1.51327800 & -3.01604700 & -0.07079300 \\ \mathrm{C} & 1.94138400 & -1.57520900 & -0.19114500 \\ \mathrm{C} & 0.92170700 & -0.60888900 & -0.06341200 \\ \mathrm{C} & 1.25993000 & 0.79213000 & 0.12313200\end{array}$




$\begin{array}{lrrr}\mathrm{C} & -0.32107100 & -1.62969000 & -2.32853200 \\ \mathrm{H} & -3.91043000 & 2.18422300 & -2.07163100 \\ \mathrm{H} & -4.73584100 & 2.05292900 & 0.25353800 \\ \mathrm{H} & -3.66267200 & 0.56719900 & 1.92085600 \\ \mathrm{H} & -1.95368700 & 0.83736500 & -2.79672200 \\ \mathrm{H} & 0.22952800 & -3.16388200 & 1.67687900 \\ \mathrm{H} & -0.55616200 & -3.74205800 & 0.20972600 \\ \mathrm{H} & 1.47897700 & -3.51639700 & -1.04519300 \\ \mathrm{H} & 2.23862300 & -3.57606300 & 0.52657400 \\ \mathrm{H} & -1.20305300 & -2.18870400 & -2.66131800 \\ \mathrm{H} & -0.14167700 & -0.83059000 & -3.05263500 \\ \mathrm{H} & 0.53626000 & -2.29661900 & -2.35038300 \\ \mathrm{C} & -1.52837300 & -1.21374600 & 2.64230300 \\ \mathrm{H} & -0.84052800 & -1.98537600 & 2.98280800 \\ \mathrm{H} & -1.26950300 & -0.27010800 & 3.13453300 \\ \mathrm{H} & -2.54109200 & -1.50605000 & 2.93257700 \\ \mathrm{C} & 2.65413600 & 1.14094600 & -0.00046800 \\ \mathrm{C} & 3.28982700 & -1.19826000 & -0.28133800 \\ \mathrm{H} & 4.05678300 & -1.96215600 & -0.36537900 \\ \mathrm{C} & 3.63051400 & 0.13644700 & -0.22388900 \\ \mathrm{H} & 4.67203300 & 0.43506900 & -0.30278000 \\ \mathrm{C} & 3.06561500 & 2.49452800 & 0.13550300 \\ \mathrm{H} & 4.12153600 & 2.72598200 & 0.02995100 \\ \mathrm{C} & 2.15487200 & 3.48966200 & 0.40896600 \\ \mathrm{H} & 2.47713100 & 4.52001100 & 0.51674900 \\ \mathrm{H} & -0.69364400 & 1.65042800 & 0.55353700 \\ \mathrm{C} & 0.79465400 & 3.15045900 & 0.55852000 \\ \mathrm{C} & 0.35753500 & 1.84445900 & 0.41709200 \\ \mathrm{H} & 0.07089300 & 3.92530300 & 0.79219400 \\ & & & \\ & & & \\ & & & \end{array}$

\section{2y-TS1b}

$\begin{array}{lrrc}\mathrm{C} & -4.83995100 & -0.58859900 & 0.74131100 \\ \mathrm{C} & -4.62911700 & -1.38573500 & -0.40627000 \\ \mathrm{C} & -3.39858600 & -1.43814100 & -1.05510400 \\ \mathrm{C} & -2.36161700 & -0.66829200 & -0.51456300 \\ \mathrm{C} & -2.56270500 & 0.15432500 & 0.62269400 \\ \mathrm{C} & -3.81621100 & 0.18919100 & 1.26222600 \\ \mathrm{~N} & -1.04457300 & -0.54614900 & -0.93547400 \\ \mathrm{C} & -0.40083300 & 0.39104500 & -0.13872400 \\ \mathrm{C} & -1.31213300 & 0.80276900 & 0.90198600 \\ \mathrm{C} & 1.07059800 & 0.58029800 & -0.16000000 \\ \mathrm{C} & 1.49787100 & 1.86556800 & -0.44589600 \\ \mathrm{C} & 0.40182000 & 2.88657800 & -0.64161400 \\ \mathrm{C} & -0.95775500 & 2.19986500 & -0.62579000\end{array}$




$\begin{array}{lrrc}\mathrm{C} & 2.00094700 & -0.47088700 & 0.12611800 \\ \mathrm{C} & -0.94539700 & 1.50606500 & 2.16915000 \\ \mathrm{H} & -5.81639600 & -0.58580200 & 1.21355400 \\ \mathrm{H} & -5.45269600 & -1.97764600 & -0.79331200 \\ \mathrm{H} & -3.25701600 & -2.05728000 & -1.93389700 \\ \mathrm{H} & -3.97843800 & 0.80567600 & 2.14076900 \\ \mathrm{H} & 0.51740800 & 3.41972500 & -1.59498500 \\ \mathrm{H} & 0.45346500 & 3.64869400 & 0.14469300 \\ \mathrm{H} & -1.34974900 & 1.87860700 & -1.58974600 \\ \mathrm{H} & -1.72649600 & 2.75537000 & -0.09326500 \\ \mathrm{H} & -1.68017900 & 2.27266100 & 2.43288500 \\ \mathrm{H} & -0.93413400 & 0.77097700 & 2.98230800 \\ \mathrm{H} & 0.04604800 & 1.95830600 & 2.11802000 \\ \mathrm{C} & 3.39890500 & -0.14980700 & 0.06559700 \\ \mathrm{C} & 2.87835700 & 2.17062800 & -0.49337000 \\ \mathrm{H} & 3.20339600 & 3.18033100 & -0.72554500 \\ \mathrm{C} & 3.80116300 & 1.17765700 & -0.25413000 \\ \mathrm{C} & -0.53905100 & -1.08232600 & -2.19785800 \\ \mathrm{H} & 0.52340200 & -0.85877800 & -2.28045900 \\ \mathrm{H} & -1.07060200 & -0.64112100 & -3.04806000 \\ \mathrm{H} & -0.66963100 & -2.16694300 & -2.21624600 \\ \mathrm{H} & 4.86319700 & 1.40123500 & -0.30069500 \\ \mathrm{C} & 4.35319600 & -1.16547200 & 0.34295600 \\ \mathrm{H} & 5.40943400 & -0.91714900 & 0.29163100 \\ \mathrm{C} & 3.95209900 & -2.44004700 & 0.68007000 \\ \mathrm{H} & 4.69016000 & -3.20655600 & 0.89315900 \\ \mathrm{C} & 2.57378800 & -2.75136400 & 0.75644000 \\ \mathrm{C} & 1.61933100 & -1.79206900 & 0.48490200 \\ \mathrm{H} & 0.56708600 & -2.05017900 & 0.55254600 \\ \mathrm{H} & 2.26542800 & -3.75458500 & 1.03417600 \\ & & & \\ & & & \\ \mathrm{H} & & \end{array}$

$\begin{array}{lrrr}\text { 2z-TS1a } & & & \\ \mathrm{C} & 1.72676300 & 3.69017600 & 0.00517500 \\ \mathrm{C} & 0.32173400 & 3.61884800 & 0.03964500 \\ \mathrm{C} & -0.36523400 & 2.41062500 & -0.07648900 \\ \mathrm{C} & 0.40137100 & 1.25273300 & -0.23971200 \\ \mathrm{C} & 1.81239900 & 1.31047400 & -0.26458500 \\ \mathrm{C} & 2.48423500 & 2.53405200 & -0.14298800 \\ \mathrm{~N} & 0.01357900 & -0.10318100 & -0.38335900 \\ \mathrm{C} & 1.16651700 & -0.89676300 & -0.51320600 \\ \mathrm{C} & 2.32113800 & -0.03863300 & -0.47465200 \\ \mathrm{C} & 1.22154900 & -2.26937900 & -1.15569000 \\ \mathrm{C} & 2.15305500 & -3.14646100 & -0.27769100 \\ \mathrm{C} & 2.38924900 & -2.37798400 & 0.98244900\end{array}$




$\begin{array}{lrrc}\mathrm{C} & 2.05717700 & -1.06905200 & 1.02990200 \\ \mathrm{C} & 1.88487000 & -0.24074100 & 2.26916300 \\ \mathrm{C} & 3.67117400 & -0.29050300 & -1.09049000 \\ \mathrm{H} & 2.21753100 & 4.65356600 & 0.09258300 \\ \mathrm{H} & -0.25098400 & 4.53409800 & 0.15258700 \\ \mathrm{H} & -1.44406200 & 2.37642700 & -0.05835200 \\ \mathrm{H} & 3.56824900 & 2.58019100 & -0.17102700 \\ \mathrm{H} & 0.22594900 & -2.70303100 & -1.20540800 \\ \mathrm{H} & 1.60137200 & -2.15872700 & -2.17665800 \\ \mathrm{H} & 3.09552400 & -3.40954900 & -0.77675700 \\ \mathrm{H} & 1.67516600 & -4.10145000 & -0.03323200 \\ \mathrm{H} & 2.70702000 & -2.89161600 & 1.88775400 \\ \mathrm{H} & 2.62931000 & 0.55553100 & 2.35084600 \\ \mathrm{H} & 0.89551000 & 0.22689400 & 2.29758200 \\ \mathrm{H} & 1.96335600 & -0.88865400 & 3.14622800 \\ \mathrm{H} & 3.68988900 & 0.17380000 & -2.08306900 \\ \mathrm{H} & 4.46103300 & 0.17043600 & -0.49188300 \\ \mathrm{H} & 3.90127100 & -1.34879300 & -1.20317900 \\ \mathrm{C} & -1.26626900 & -0.69016600 & -0.10906700 \\ \mathrm{O} & -2.22185400 & 0.21034600 & -0.22169800 \\ \mathrm{O} & -1.35982600 & -1.86643100 & 0.17026400 \\ \mathrm{C} & -3.68264500 & -0.14761600 & -0.00203900 \\ \mathrm{C} & -3.86726200 & -0.64166500 & 1.43158500 \\ \mathrm{C} & -4.09803700 & -1.17471000 & -1.05353700 \\ \mathrm{C} & -4.37272400 & 1.19553500 & -0.22713600 \\ \mathrm{H} & -3.37602800 & -1.60098100 & 1.59946900 \\ \mathrm{H} & -3.48592900 & 0.09191100 & 2.14874100 \\ \mathrm{H} & -4.93747100 & -0.76779900 & 1.62211800 \\ \mathrm{H} & -3.88861300 & -0.80483800 & -2.06183700 \\ \mathrm{H} & -3.59620200 & -2.13218100 & -0.90675700 \\ \mathrm{H} & -5.17697800 & -1.33971900 & -0.97521800 \\ \mathrm{H} & -5.45341400 & 1.06920900 & -0.11806100 \\ & & 1.93702400 & 0.50733600 \\ \mathrm{H} & & & -1.23265100\end{array}$

\section{2z-TS1b}

$\begin{array}{lrrr}\mathrm{C} & -1.68117400 & 3.87679900 & -0.01702500 \\ \mathrm{C} & -0.27836000 & 3.76269600 & -0.00733600 \\ \mathrm{C} & 0.36979600 & 2.52841700 & -0.06320800 \\ \mathrm{C} & -0.43430300 & 1.38805600 & -0.13110200 \\ \mathrm{C} & -1.84307400 & 1.48884600 & -0.13130700 \\ \mathrm{C} & -2.47685100 & 2.73971300 & -0.07889400 \\ \mathrm{~N} & -0.09442300 & 0.01267600 & -0.19745100 \\ \mathrm{C} & -1.26889600 & -0.74613900 & -0.23013900\end{array}$




$\begin{array}{lrrc}\mathrm{C} & -2.38455900 & 0.15195000 & -0.24145000 \\ \mathrm{C} & -1.33323900 & -2.18919600 & -0.59106100 \\ \mathrm{C} & -2.02493000 & -2.94726900 & 0.26912500 \\ \mathrm{C} & -2.57782600 & -2.28587500 & 1.50059000 \\ \mathrm{C} & -2.13600800 & -0.82763300 & 1.52482500 \\ \mathrm{C} & -0.70610900 & -2.65128200 & -1.87958000 \\ \mathrm{C} & -3.78240400 & -0.16102900 & -0.67860200 \\ \mathrm{H} & -2.13956800 & 4.85909700 & 0.02176200 \\ \mathrm{H} & 0.32413600 & 4.66460500 & 0.03716800 \\ \mathrm{H} & 1.44738100 & 2.46263100 & -0.07028600 \\ \mathrm{H} & -3.55934500 & 2.81793800 & -0.09014900 \\ \mathrm{H} & -2.20091200 & -4.00410900 & 0.09281900 \\ \mathrm{H} & -2.21491000 & -2.78089800 & 2.41205000 \\ \mathrm{H} & -3.67195800 & -2.36242400 & 1.52647300 \\ \mathrm{H} & -1.18803100 & -0.62437700 & 2.02143100 \\ \mathrm{H} & -2.88804800 & -0.12811500 & 1.88562500 \\ \mathrm{H} & -1.07582500 & -2.07466000 & -2.73522100 \\ \mathrm{H} & 0.38269900 & -2.54906400 & -1.85196600 \\ \mathrm{H} & -0.93890700 & -3.70449400 & -2.05059100 \\ \mathrm{H} & -4.52103000 & 0.31074200 & -0.02379900 \\ \mathrm{H} & -3.92663500 & 0.25303700 & -1.68288300 \\ \mathrm{H} & -3.97351400 & -1.23306400 & -0.72361700 \\ \mathrm{C} & 1.17939800 & -0.58899500 & 0.09107200 \\ \mathrm{O} & 1.24393300 & -1.72887900 & 0.49349600 \\ \mathrm{O} & 2.15483500 & 0.26036600 & -0.15913800 \\ \mathrm{C} & 3.61150400 & -0.10931700 & 0.07378200 \\ \mathrm{C} & 3.97551600 & -1.27537700 & -0.84314800 \\ \mathrm{H} & 3.81574400 & -0.41969300 & 1.55528300 \\ \mathrm{H} & 4.33071500 & 1.17270400 & -0.33877200 \\ \mathrm{H} & 3.45677600 & -2.19230700 & -0.55996600 \\ \mathrm{H} & 3.74900900 & -1.03484300 & -1.88637900 \\ \mathrm{H} & 5.05218200 & -1.45617600 & -0.76848900 \\ \mathrm{H} & 4.88523000 & -0.56224800 & 1.73801800 \\ \mathrm{H} & & & 2.17852000 \\ \mathrm{H} & 3.47963500 & 0.41480500 & 1.85717700 \\ \mathrm{H} & -1.32987000 & -1.37974400 \\ \mathrm{H} & 1.42810100 & 0.30155800 \\ \mathrm{H} & 2.01201800 & -0.24054600\end{array}$


8. NMR Spectra for Synthesized Compounds

${ }^{1} \mathrm{H} \mathrm{NMR}\left(\mathrm{CDCl}_{3}, 400 \mathrm{MHz}\right)$ of $\mathbf{S 2}$ :

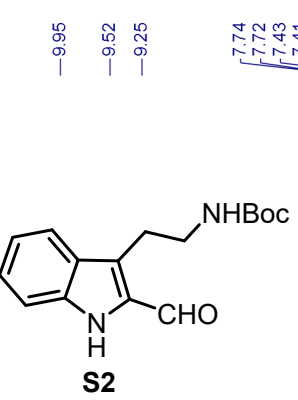

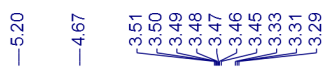
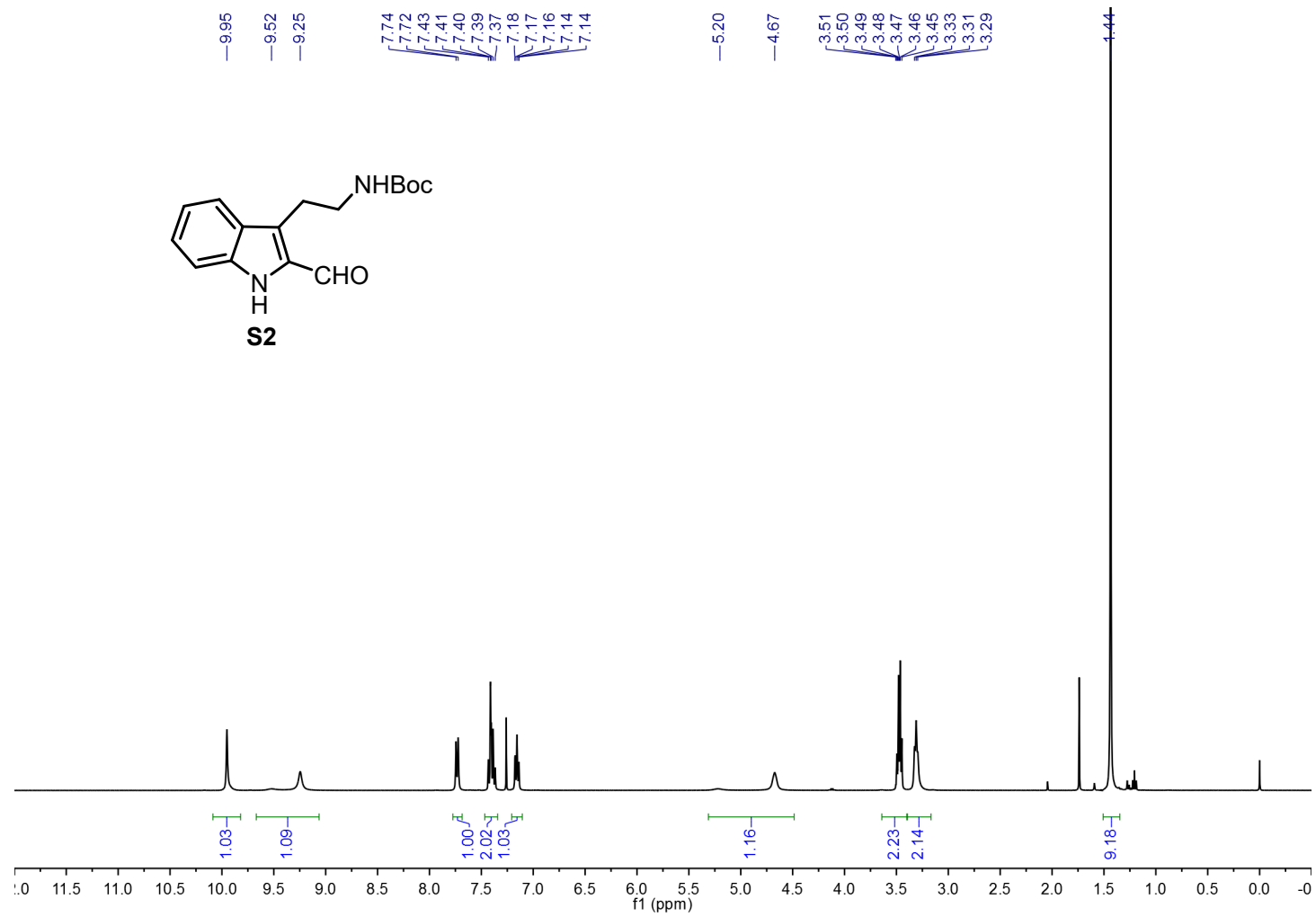

${ }^{13} \mathrm{C}\left\{{ }^{1} \mathrm{H}\right\}$ NMR $\left(\mathrm{CDCl}_{3}, 100 \mathrm{MHz}\right)$ of $\mathbf{S 2}$ :
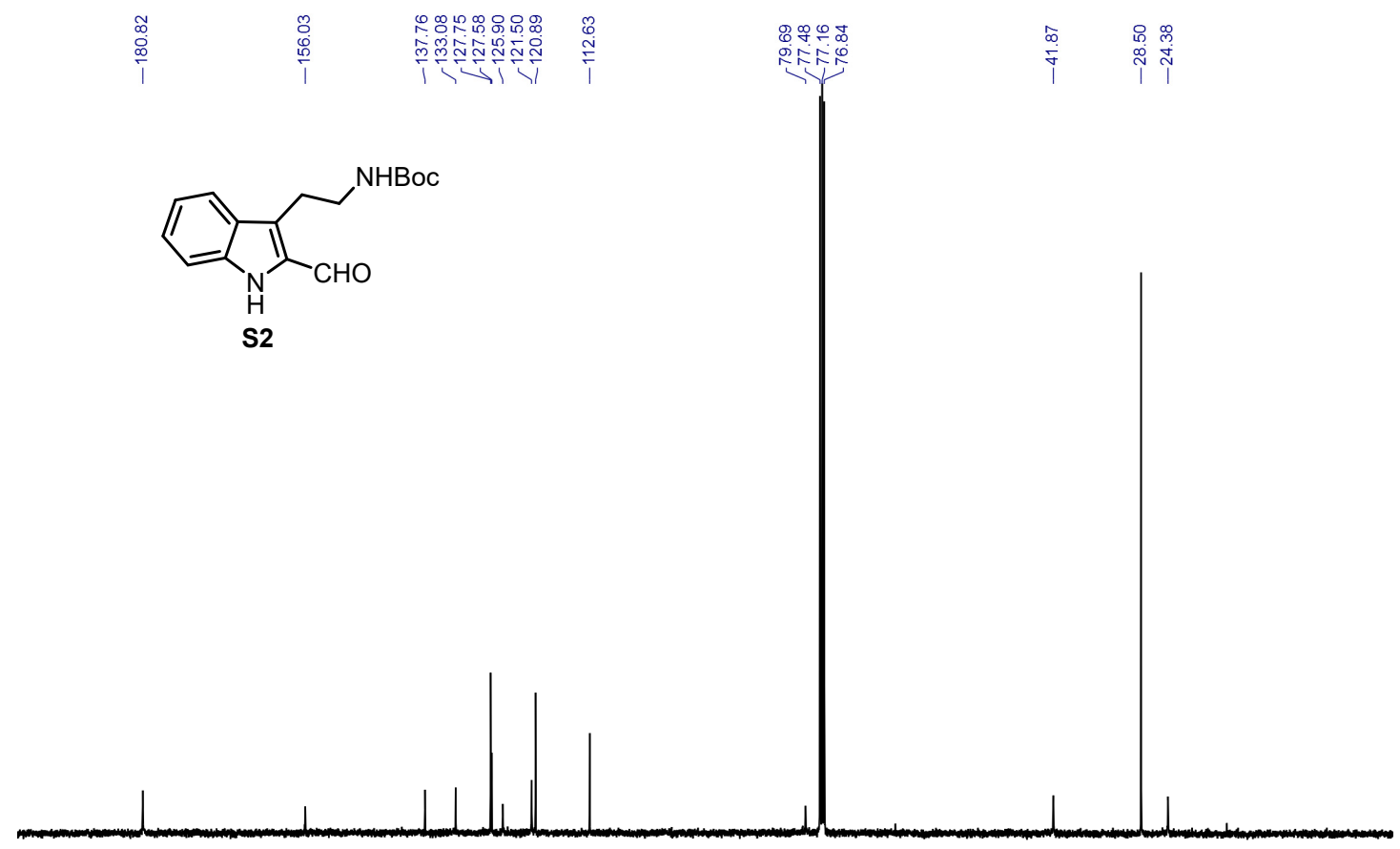

\begin{tabular}{lllllllllll}
\hline 0 & 190 & 180 & 170 & 160 & 150 & 140 & 130 & 120 & 110 & $\underset{f}{1} 100$ \\
$\mathrm{f} 1(\mathrm{ppm})$
\end{tabular} 
${ }^{1} \mathrm{H}$ NMR $\left(\mathrm{CDCl}_{3}, 400 \mathrm{MHz}\right)$ of $\mathbf{S 4}$ :

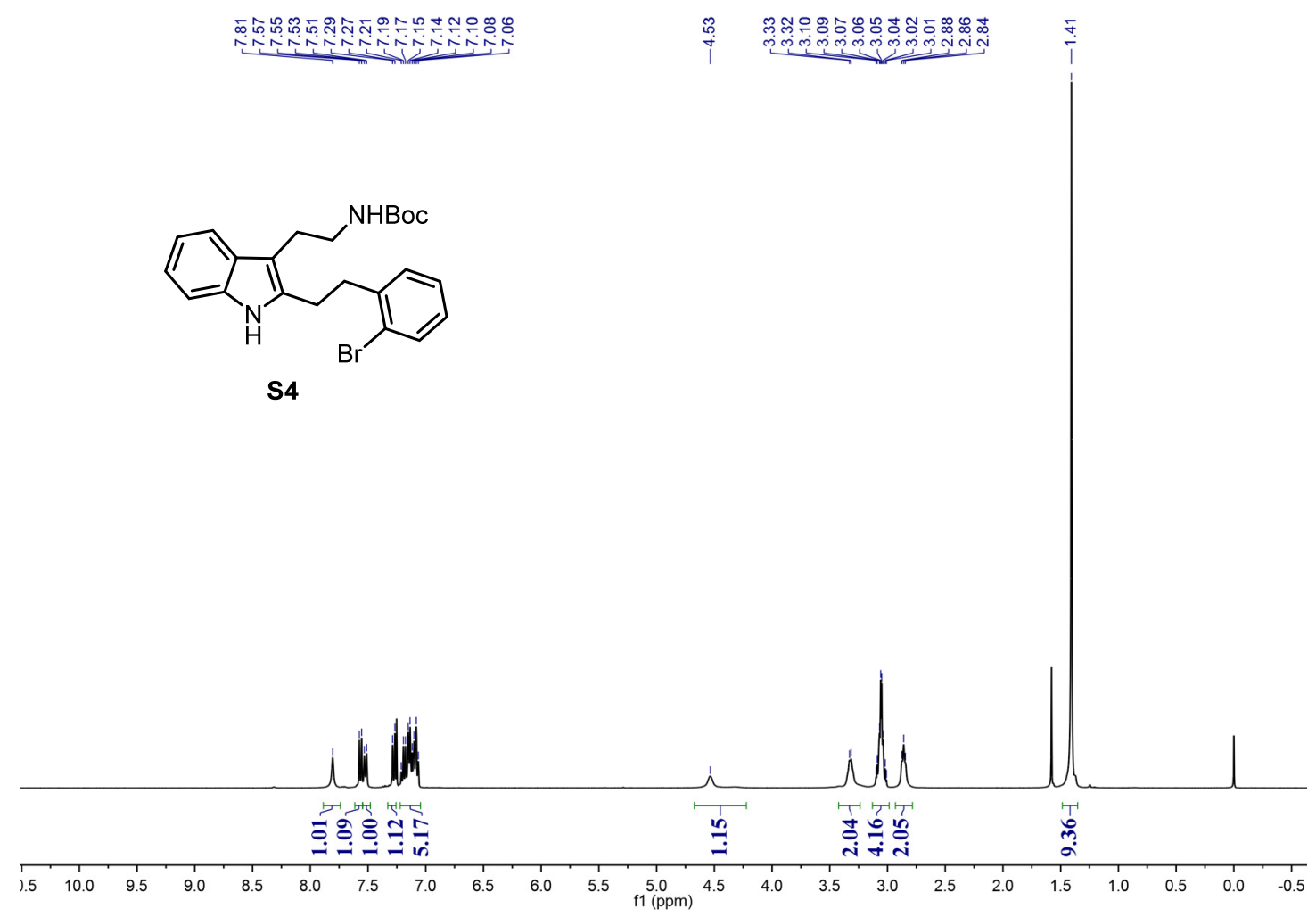

${ }^{13} \mathrm{C}\left\{{ }^{1} \mathrm{H}\right\}$ NMR $\left(\mathrm{CDCl}_{3}, 100 \mathrm{MHz}\right)$ of $\mathbf{S 4}$ :

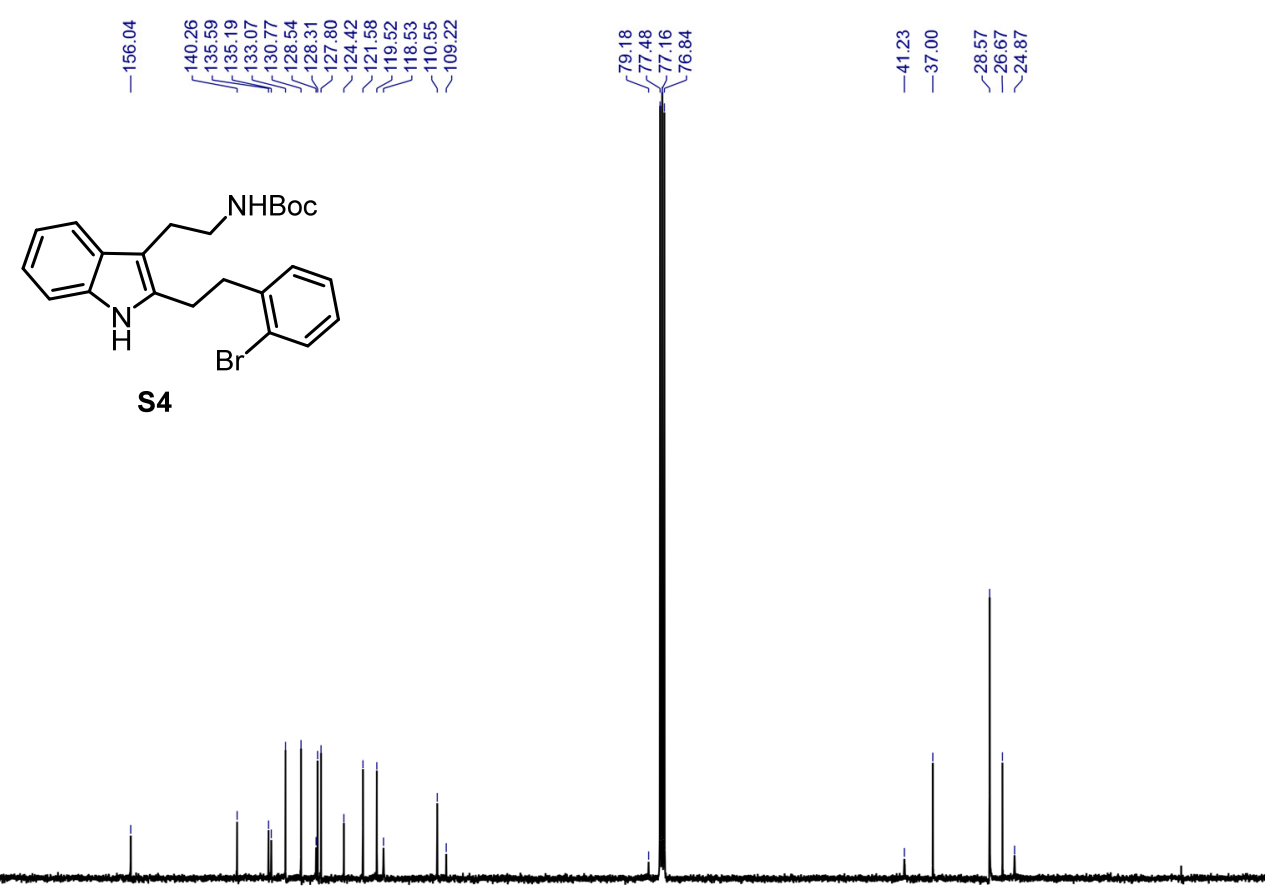

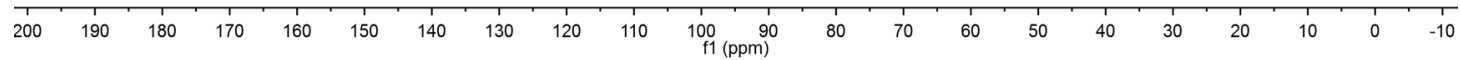


${ }^{1} \mathrm{H} \mathrm{NMR}\left(\mathrm{CDCl}_{3}, 400 \mathrm{MHz}\right)$ of $\mathbf{1 a}:$

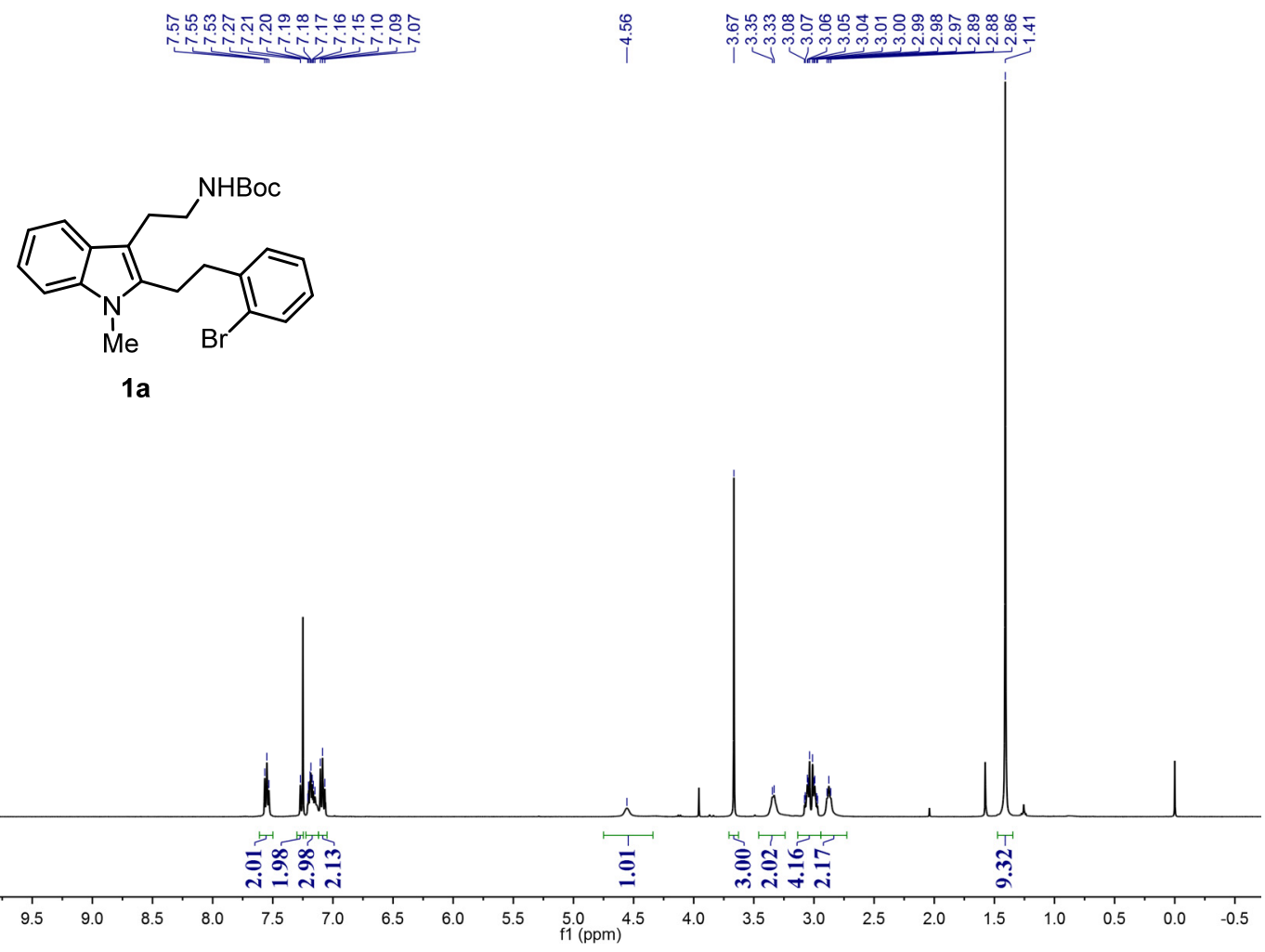

${ }^{13} \mathrm{C}\left\{{ }^{1} \mathrm{H}\right\}$ NMR $\left(\mathrm{CDCl}_{3}, 100 \mathrm{MHz}\right)$ of $\mathbf{1 a}$ :

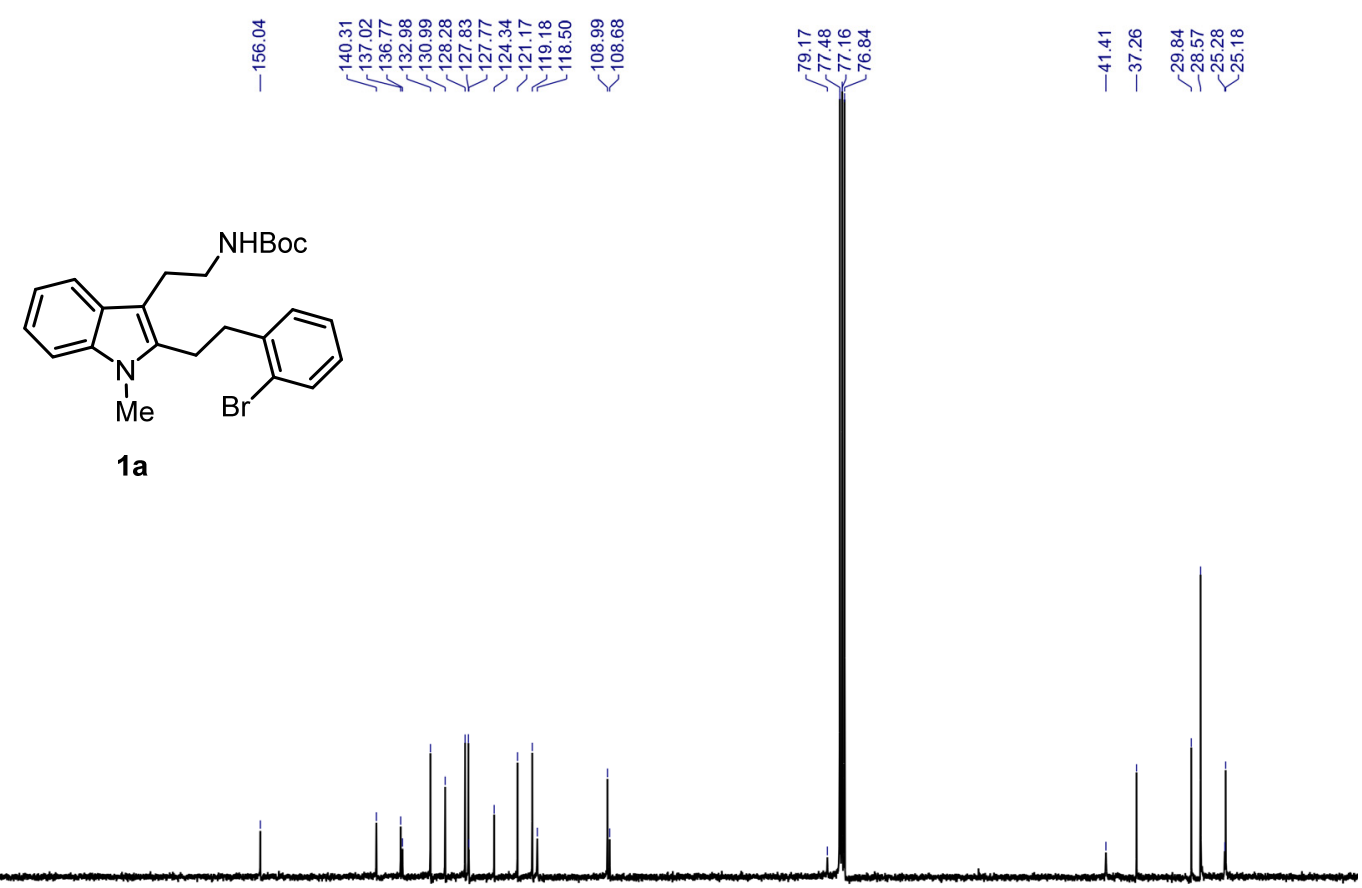

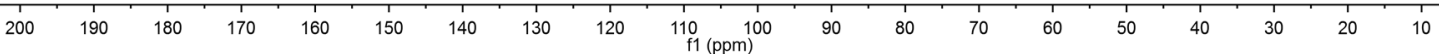


${ }^{1} \mathrm{H}$ NMR $\left(\mathrm{CDCl}_{3}, 400 \mathrm{MHz}\right)$ of $\mathbf{1 b}$ :

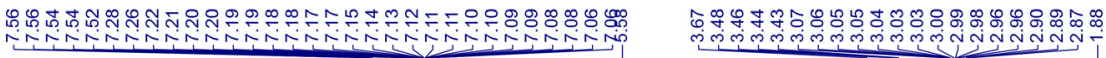

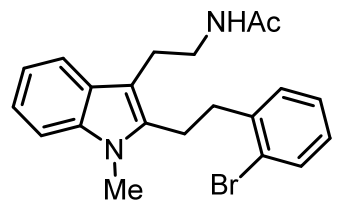

1b

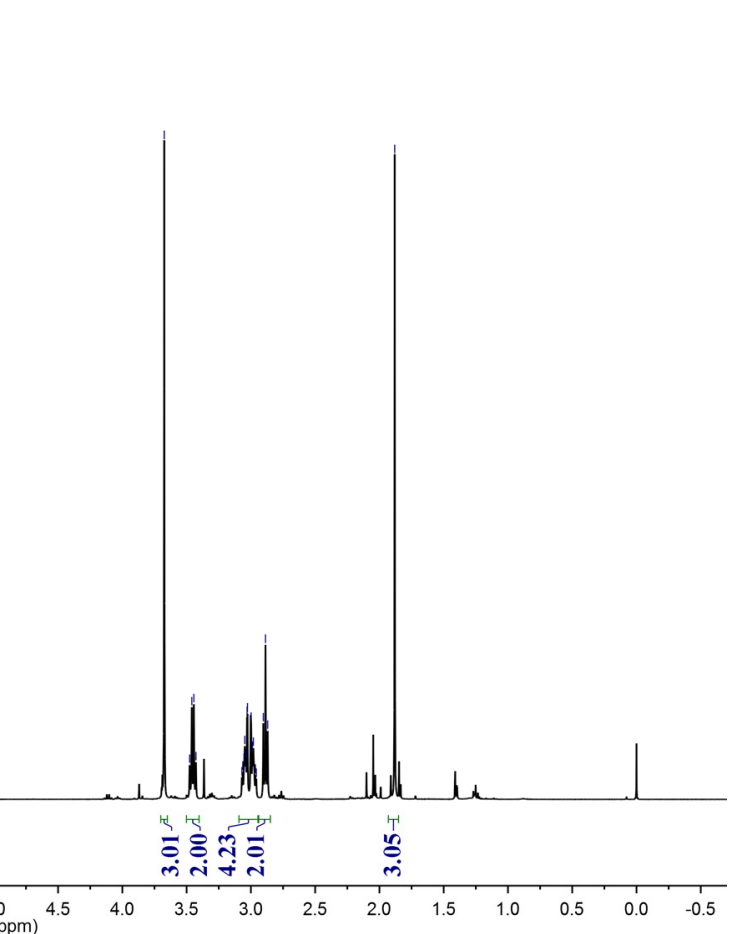

${ }^{13} \mathrm{C}\left\{{ }^{1} \mathrm{H}\right\}$ NMR $\left(\mathrm{CDCl}_{3}, 100 \mathrm{MHz}\right)$ of $\mathbf{1 b}$ :

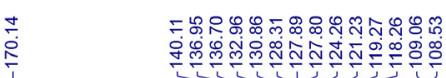

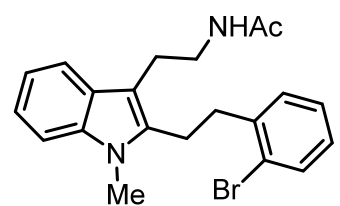

1b

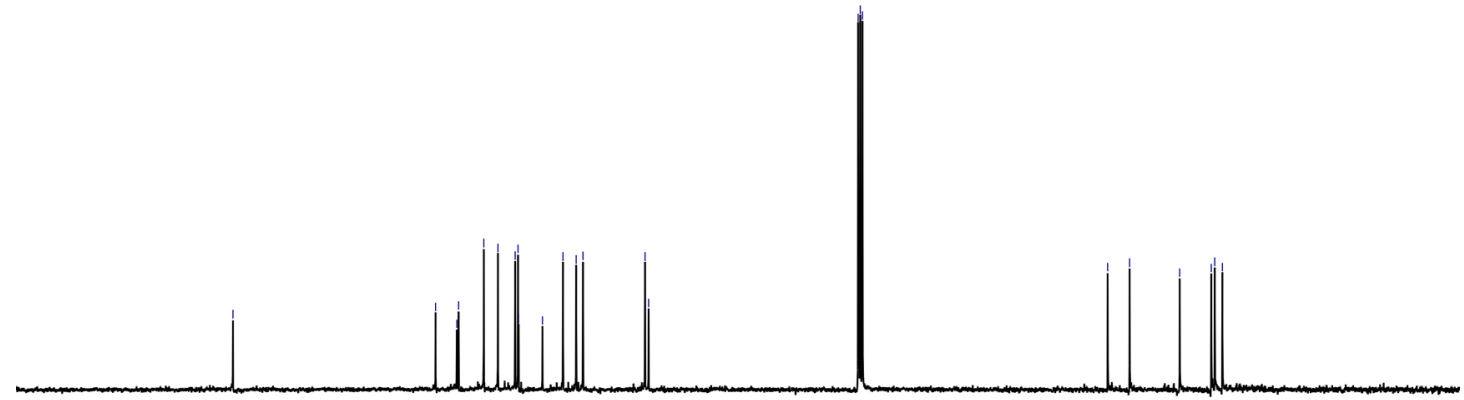

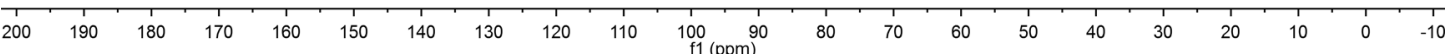


${ }^{1} \mathrm{H}$ NMR $\left(\mathrm{CDCl}_{3}, 400 \mathrm{MHz}\right)$ of $\mathbf{1 c}$ :

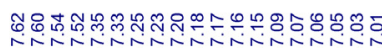

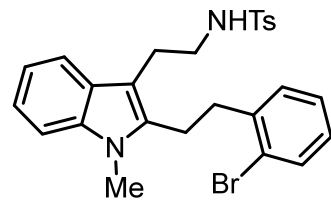

$1 \mathrm{c}$

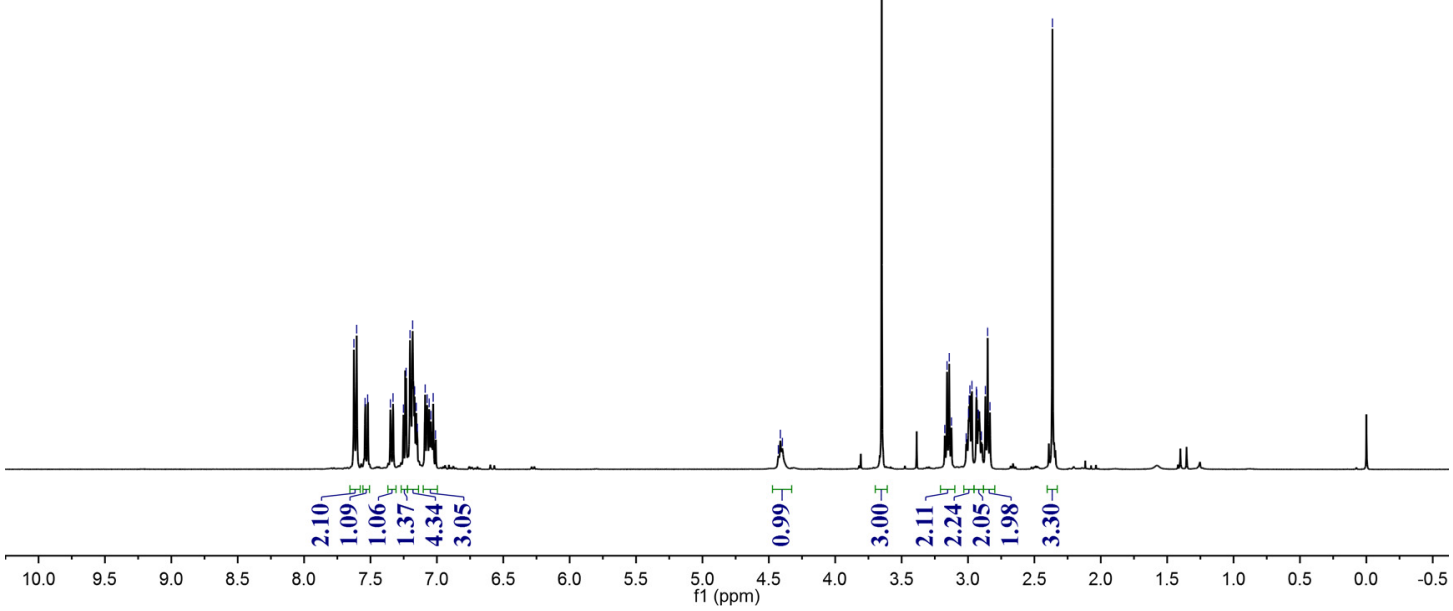

${ }^{13} \mathrm{C}\left\{{ }^{1} \mathrm{H}\right\}$ NMR $\left(\mathrm{CDCl}_{3}, 100 \mathrm{MHz}\right)$ of $\mathbf{1 c}$ :

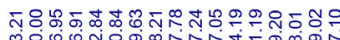

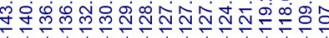

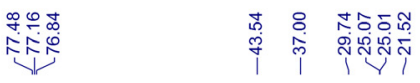

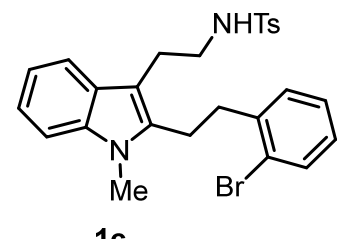

$1 \mathrm{c}$

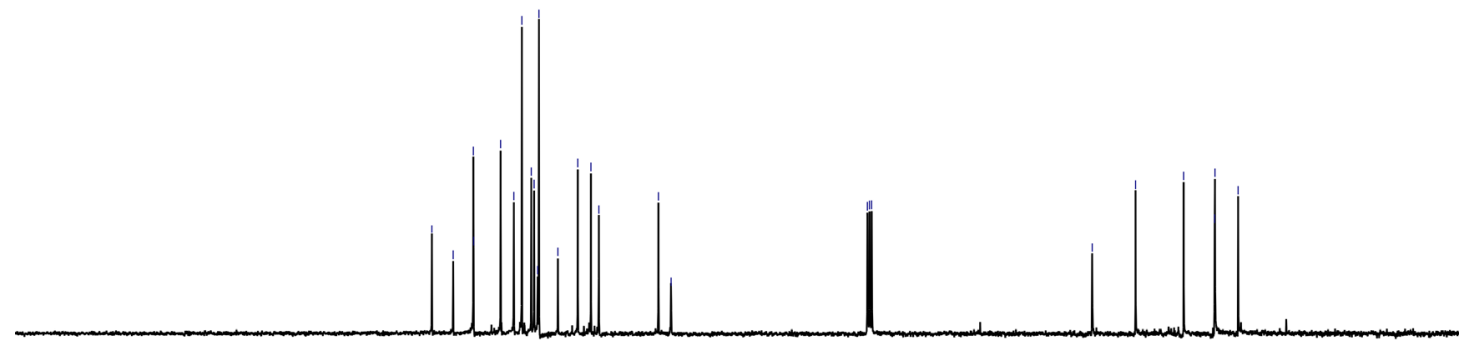

200
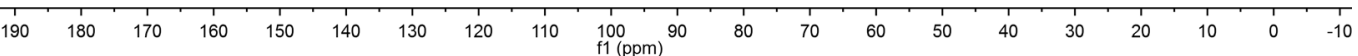
${ }^{1} \mathrm{H}$ NMR $\left(\mathrm{CDCl}_{3}, 400 \mathrm{MHz}\right)$ of $\mathbf{1 d}$ :

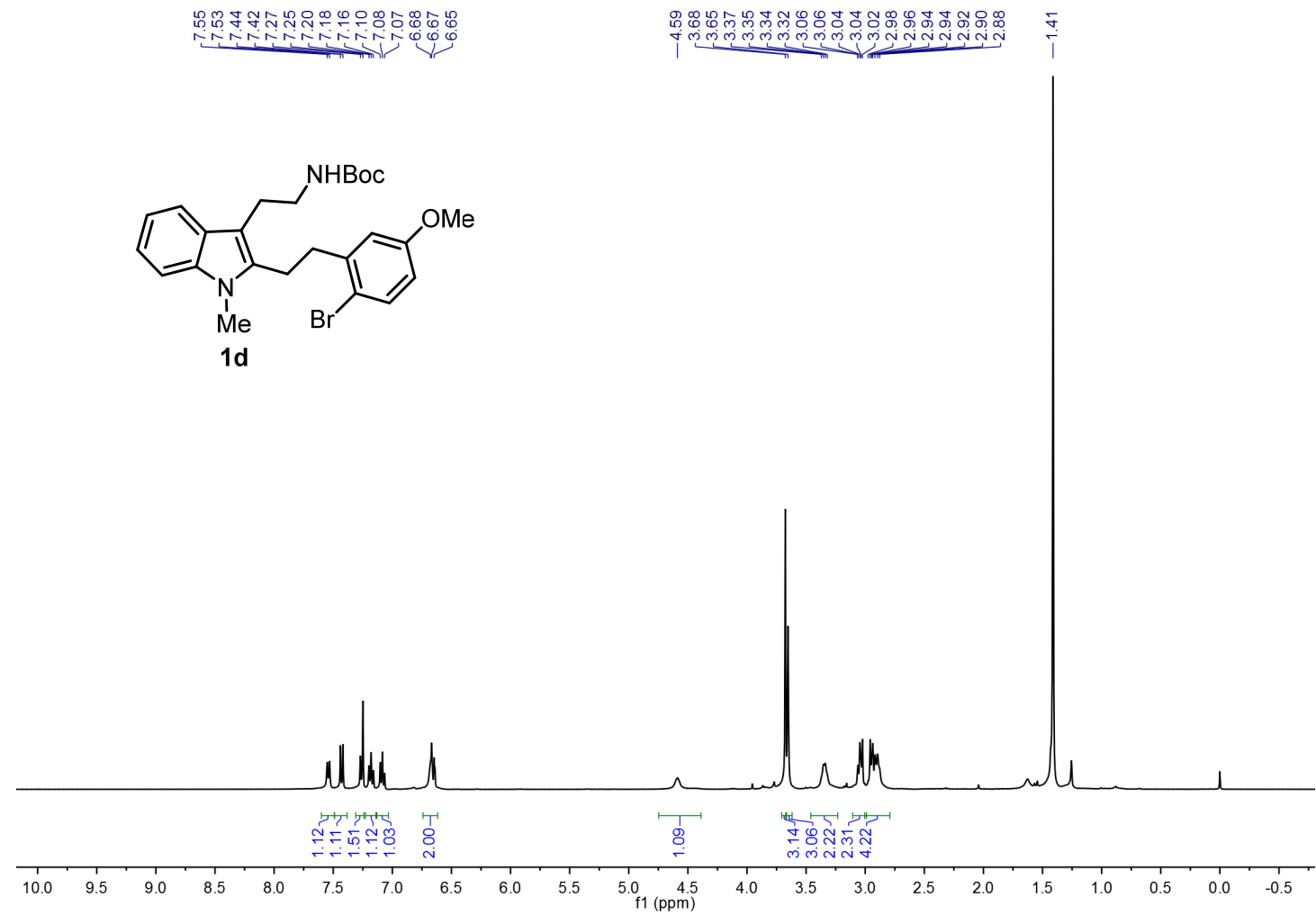

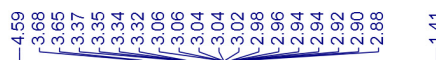

${ }^{13} \mathrm{C}\left\{{ }^{1} \mathrm{H}\right\}$ NMR $\left(\mathrm{CDCl}_{3}, 100 \mathrm{MHz}\right)$ of $\mathbf{1 d}$ :
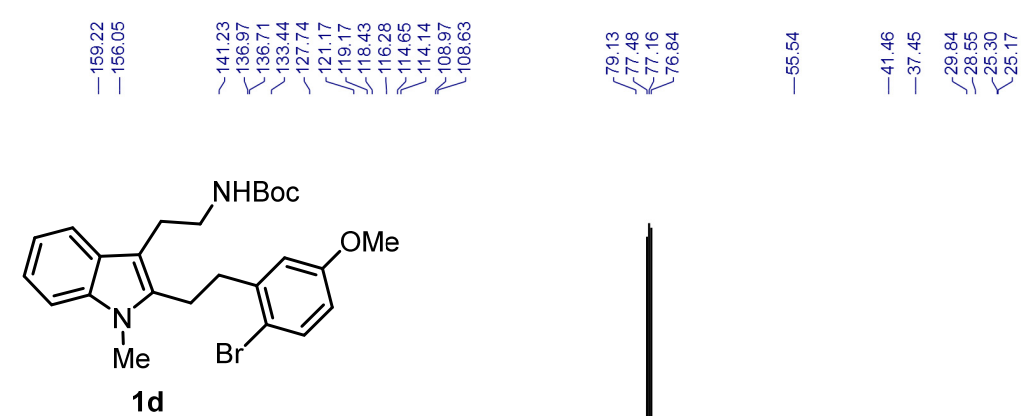

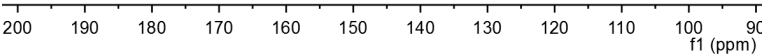

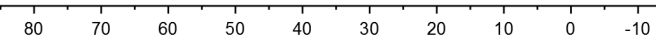


${ }^{1} \mathrm{H} \mathrm{NMR}\left(\mathrm{CDCl}_{3}, 400 \mathrm{MHz}\right)$ of $\mathbf{1 e}:$

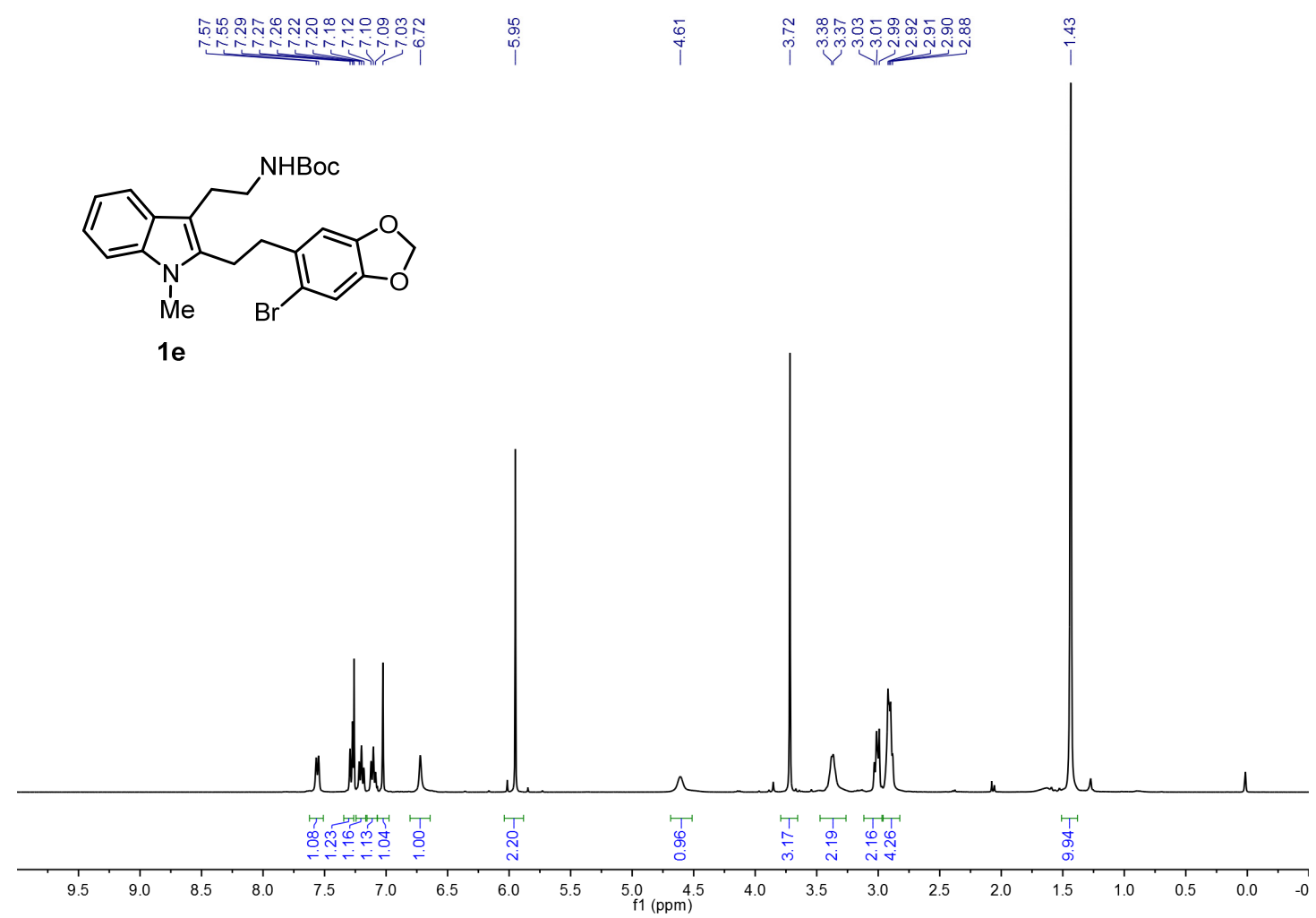

${ }^{13} \mathrm{C}\left\{{ }^{1} \mathrm{H}\right\}$ NMR $\left(\mathrm{CDCl}_{3}, 100 \mathrm{MHz}\right)$ of $\mathbf{1 e}:$

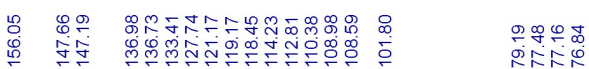

।

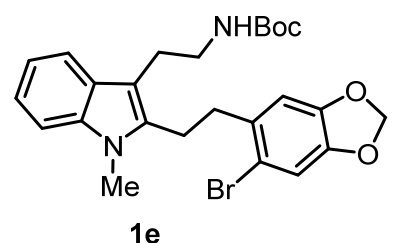

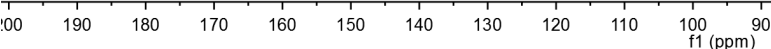

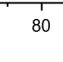


${ }^{1} \mathrm{H}$ NMR $\left(\mathrm{CDCl}_{3}, 400 \mathrm{MHz}\right)$ of $\mathbf{1 f}$ :

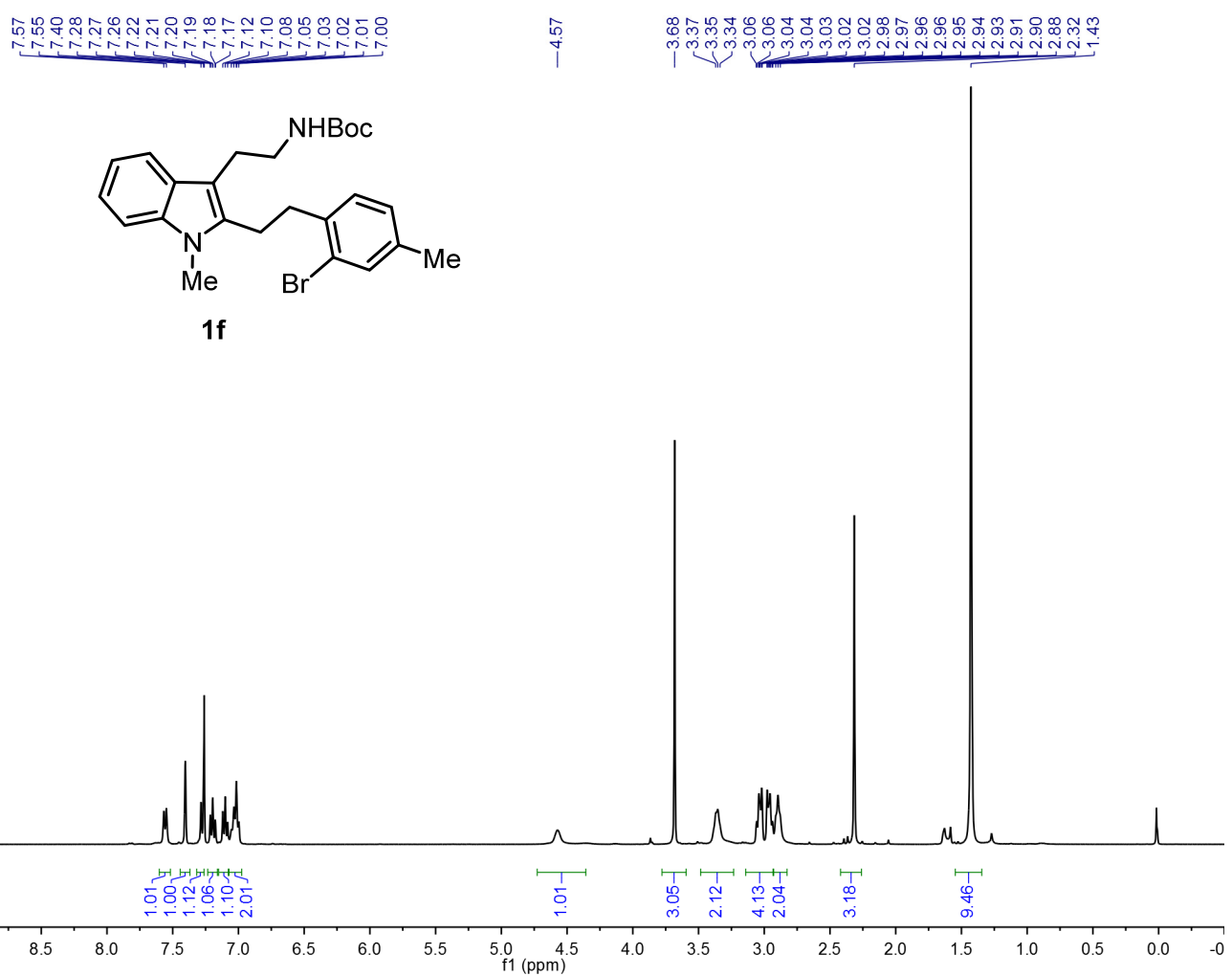

${ }^{13} \mathrm{C}\left\{{ }^{1} \mathrm{H}\right\}$ NMR $\left(\mathrm{CDCl}_{3}, 100 \mathrm{MHz}\right)$ of $\mathbf{1 f}:$

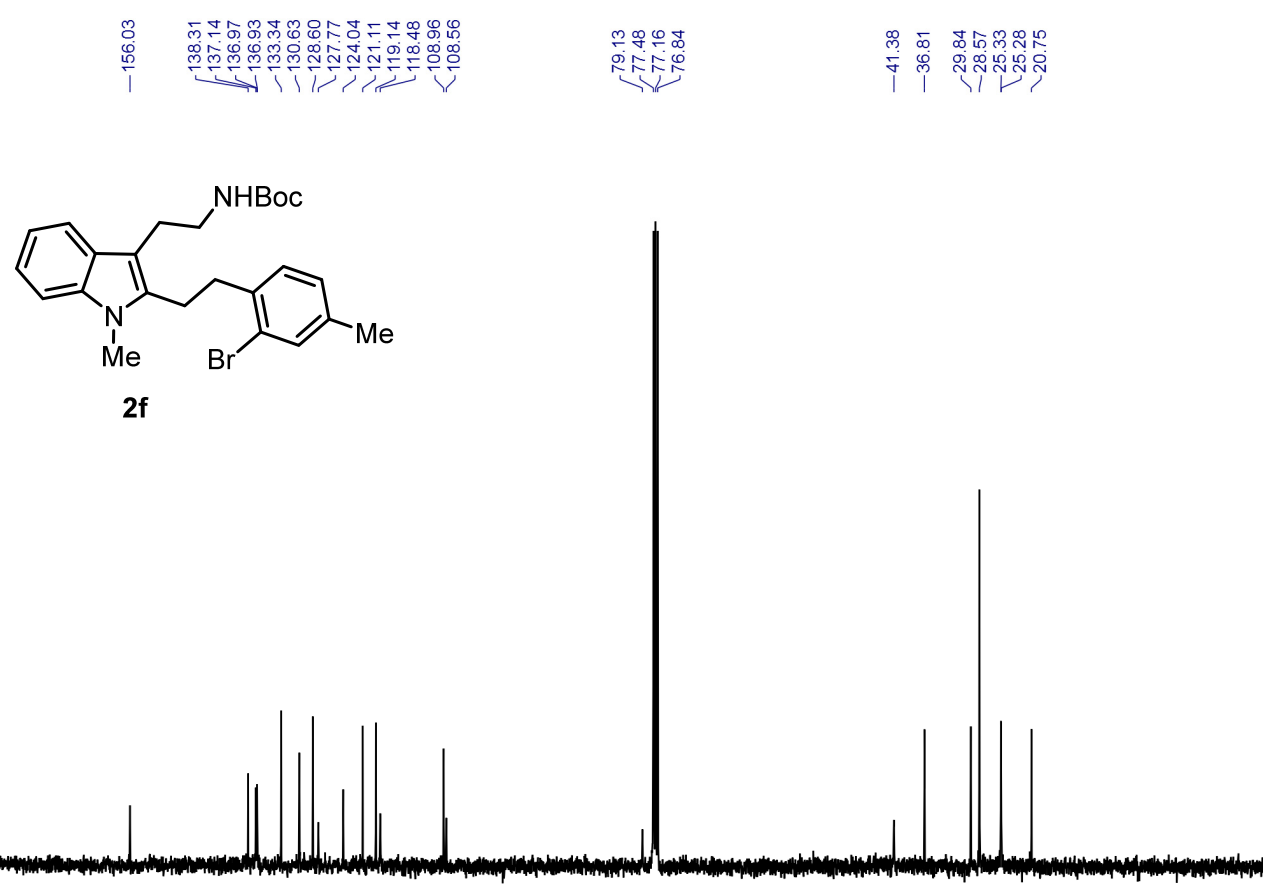

\begin{tabular}{rllllllllllllllllllll}
\hline & 1 \\
200 & 190 & 180 & 170 & 160 & 150 & 140 & 130 & 120 & 110 & $\underset{\mathrm{f} 1(\mathrm{ppm})}{90}$ & 80 & 70 & 60 & 50 & 40 & 30 & 20 & 10 & 0 & -10
\end{tabular} 
${ }^{1} \mathrm{H} \mathrm{NMR}\left(\mathrm{CDCl}_{3}, 400 \mathrm{MHz}\right)$ of $\mathbf{1 g}$ :

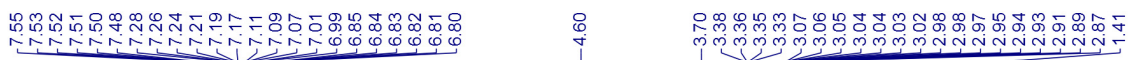

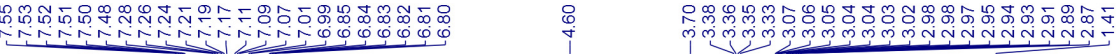

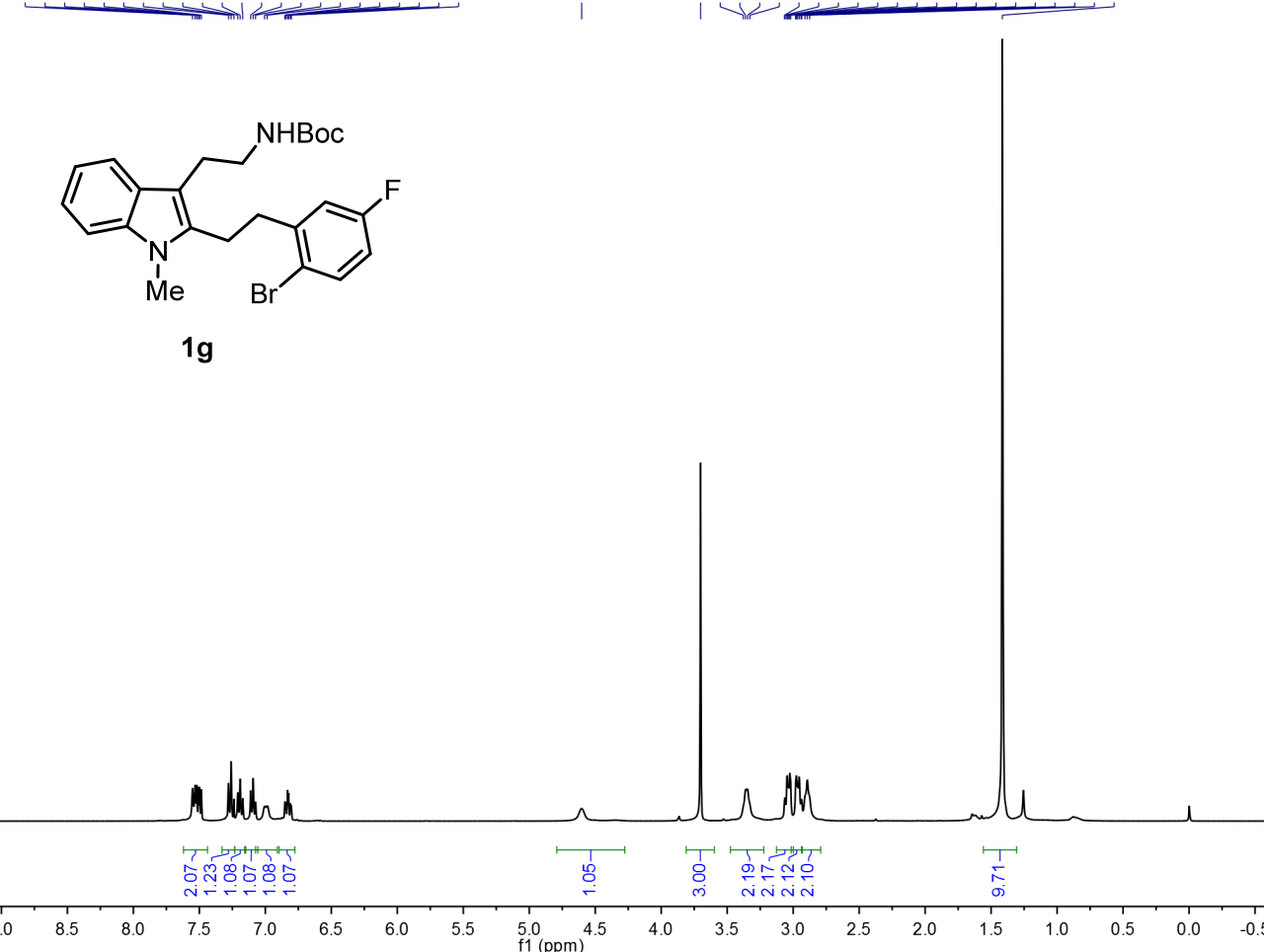

${ }^{13} \mathrm{C}\left\{{ }^{1} \mathrm{H}\right\}$ NMR $\left(\mathrm{CDCl}_{3}, 100 \mathrm{MHz}\right)$ of $\mathbf{1 g}$ :
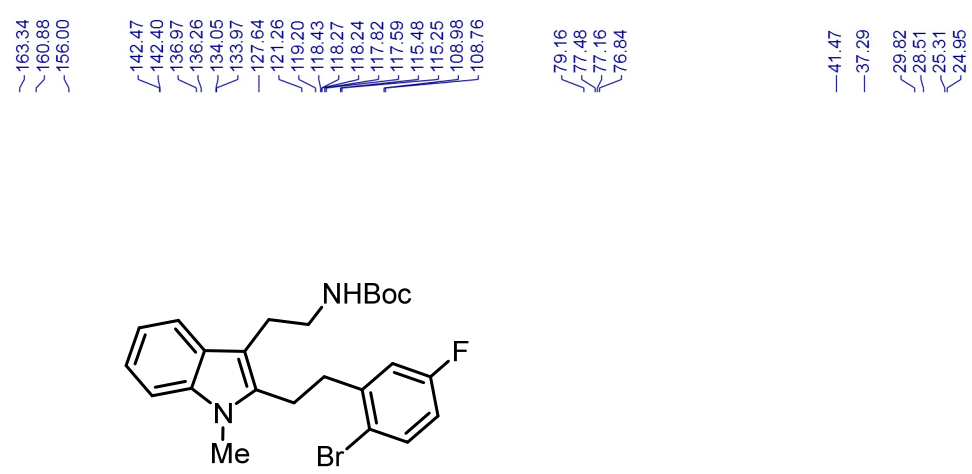

$1 \mathrm{~g}$

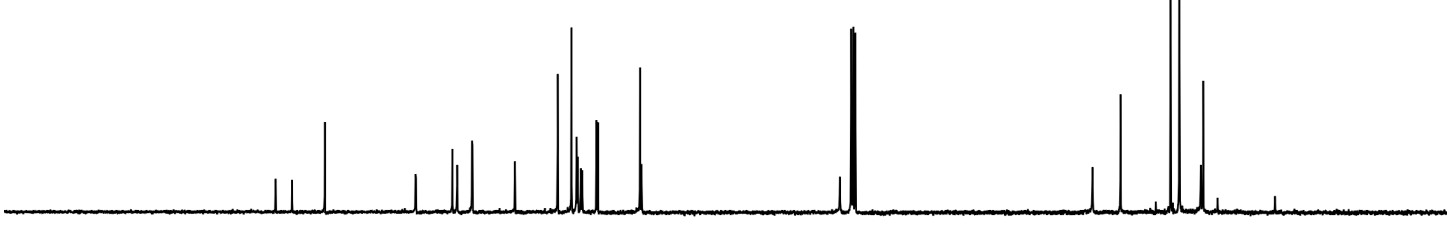

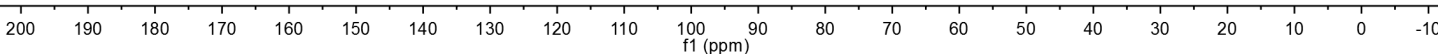


${ }^{19} \mathrm{~F}\left\{{ }^{1} \mathrm{H}\right\} \mathrm{NMR}\left(\mathrm{CDCl}_{3}, 386 \mathrm{MHz}\right)$ of $\mathbf{1 g}$ :

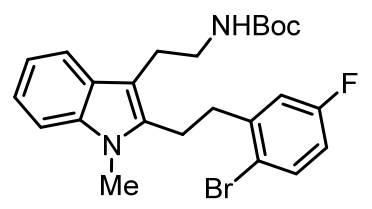



$1 \mathrm{~g}$

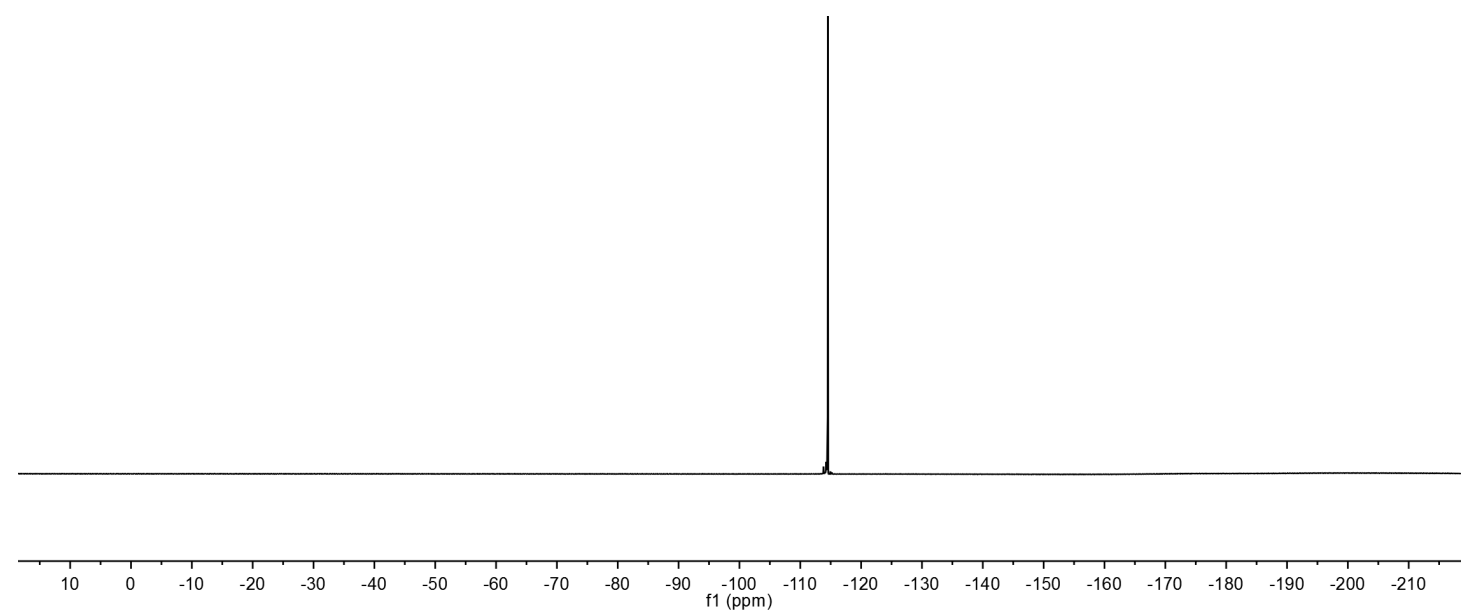


${ }^{1} \mathrm{H} \mathrm{NMR}\left(\mathrm{CDCl}_{3}, 400 \mathrm{MHz}\right)$ of $\mathbf{1 h}$ :

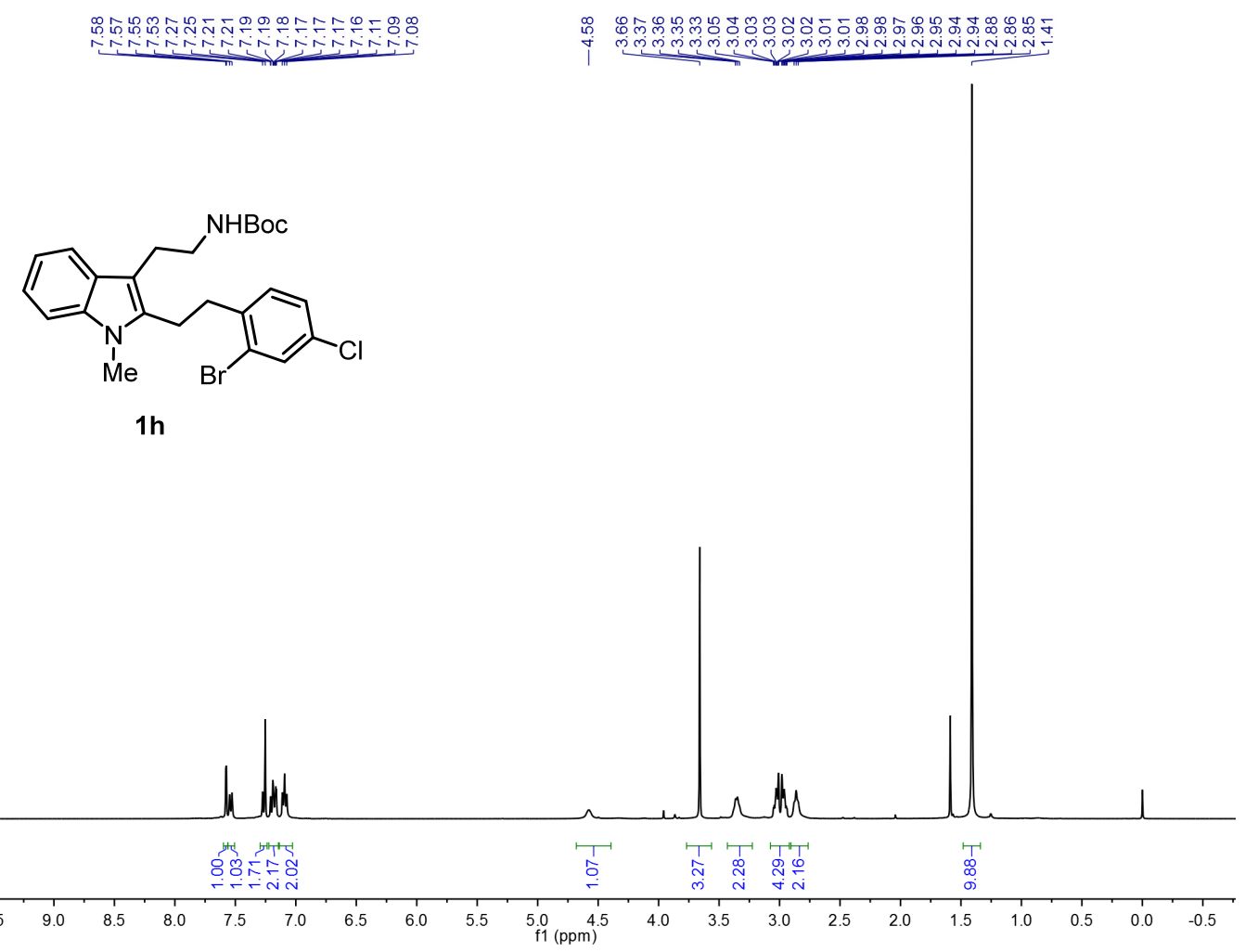

${ }^{13} \mathrm{C}\left\{{ }^{1} \mathrm{H}\right\}$ NMR $\left(\mathrm{CDCl}_{3}, 100 \mathrm{MHz}\right)$ of $\mathbf{1 h}$ :

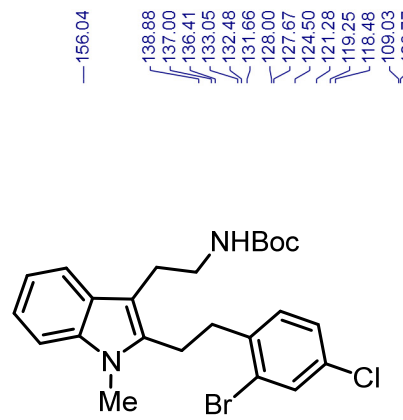

$1 \mathrm{~h}$

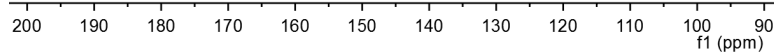

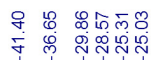

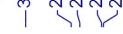

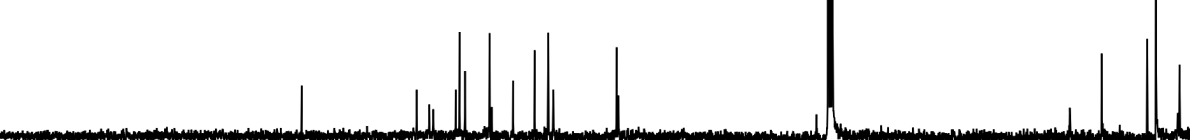


${ }^{1} \mathrm{H} \mathrm{NMR}\left(\mathrm{CDCl}_{3}, 400 \mathrm{MHz}\right)$ of $\mathbf{1 i}$ :

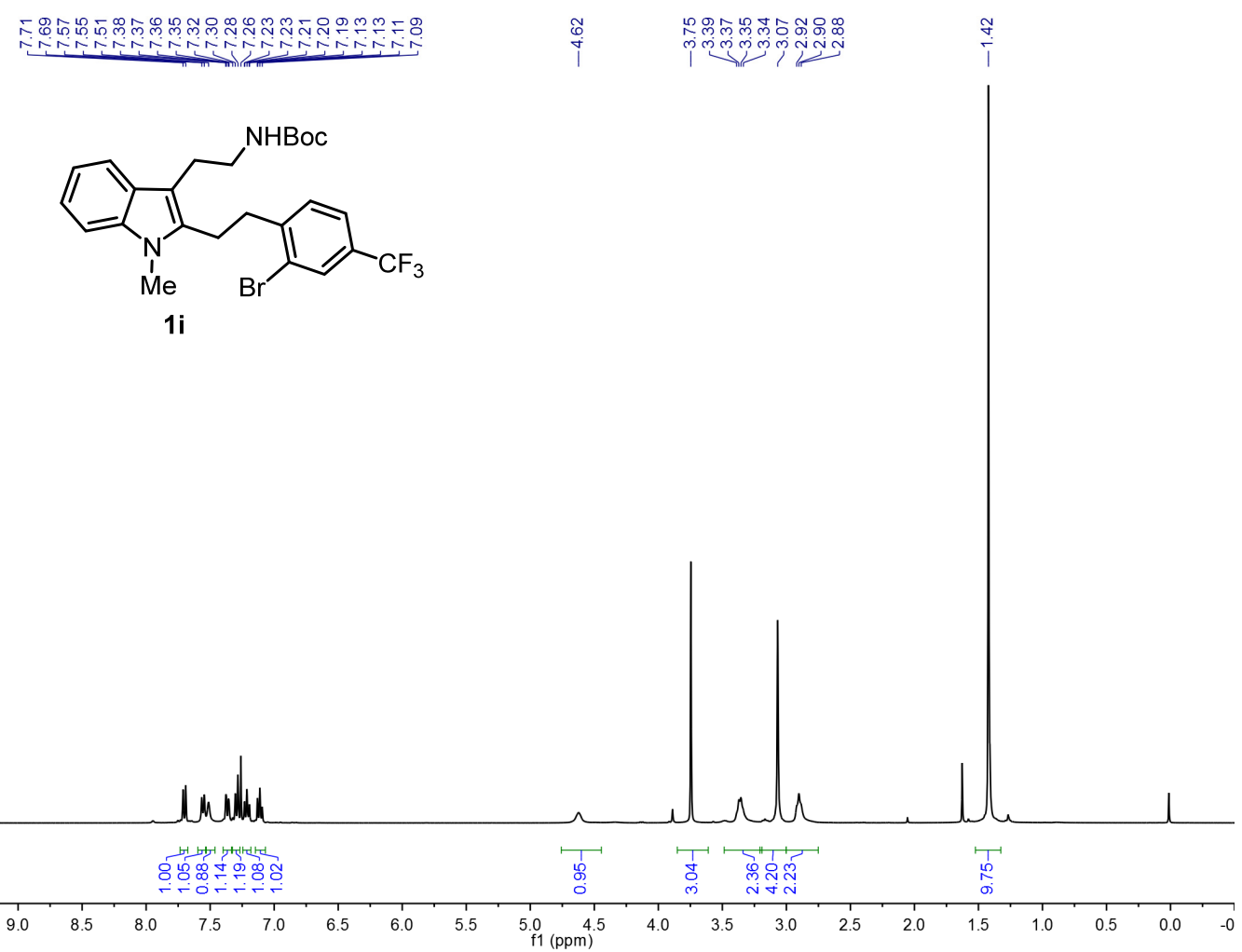

${ }^{13} \mathrm{C}\left\{{ }^{1} \mathrm{H}\right\}$ NMR $\left(\mathrm{CDCl}_{3}, 100 \mathrm{MHz}\right)$ of $\mathbf{1 i}$ :

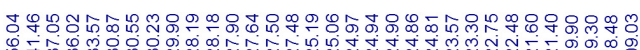

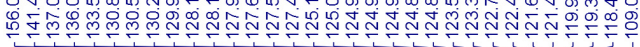

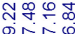

然

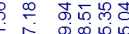

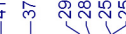

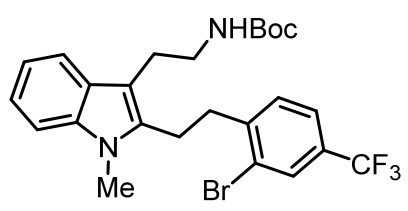

$1 \mathrm{i}$

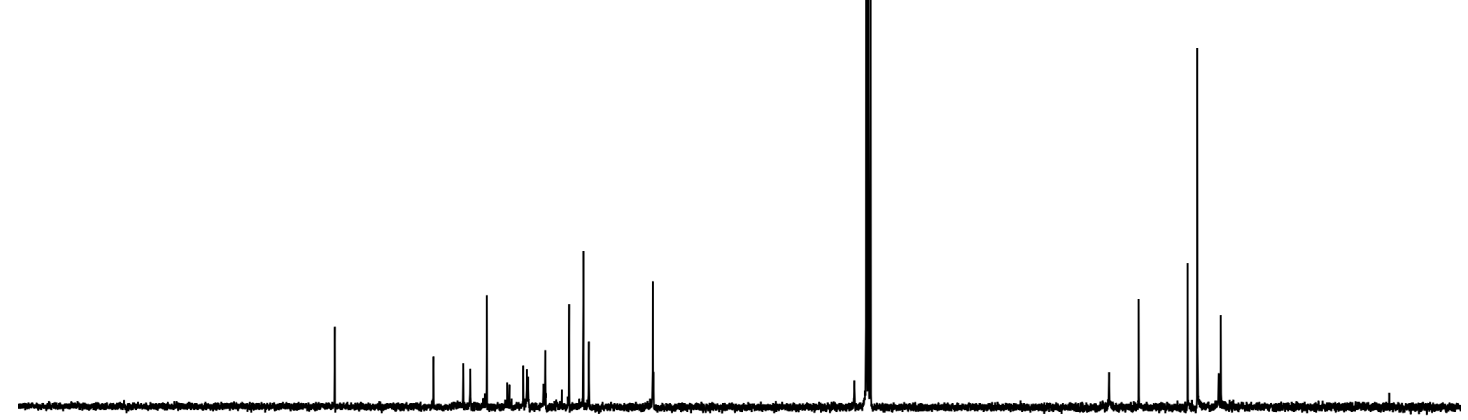

$\begin{array}{rllllllllllllllllllllll}1 & 1 \\ 200 & 190 & 180 & 170 & 160 & 150 & 140 & 130 & 120 & 110 & \underset{\mathrm{f} 1}{100}(\mathrm{ppm}) & 90 & 80 & 70 & 60 & 50 & 40 & 30 & 20 & 10 & 0 & -1\end{array}$ 
${ }^{19} \mathrm{~F}\left\{{ }^{1} \mathrm{H}\right\}$ NMR $\left(\mathrm{CDCl}_{3}, 386 \mathrm{MHz}\right)$ of $\mathbf{1 i}$ :

in
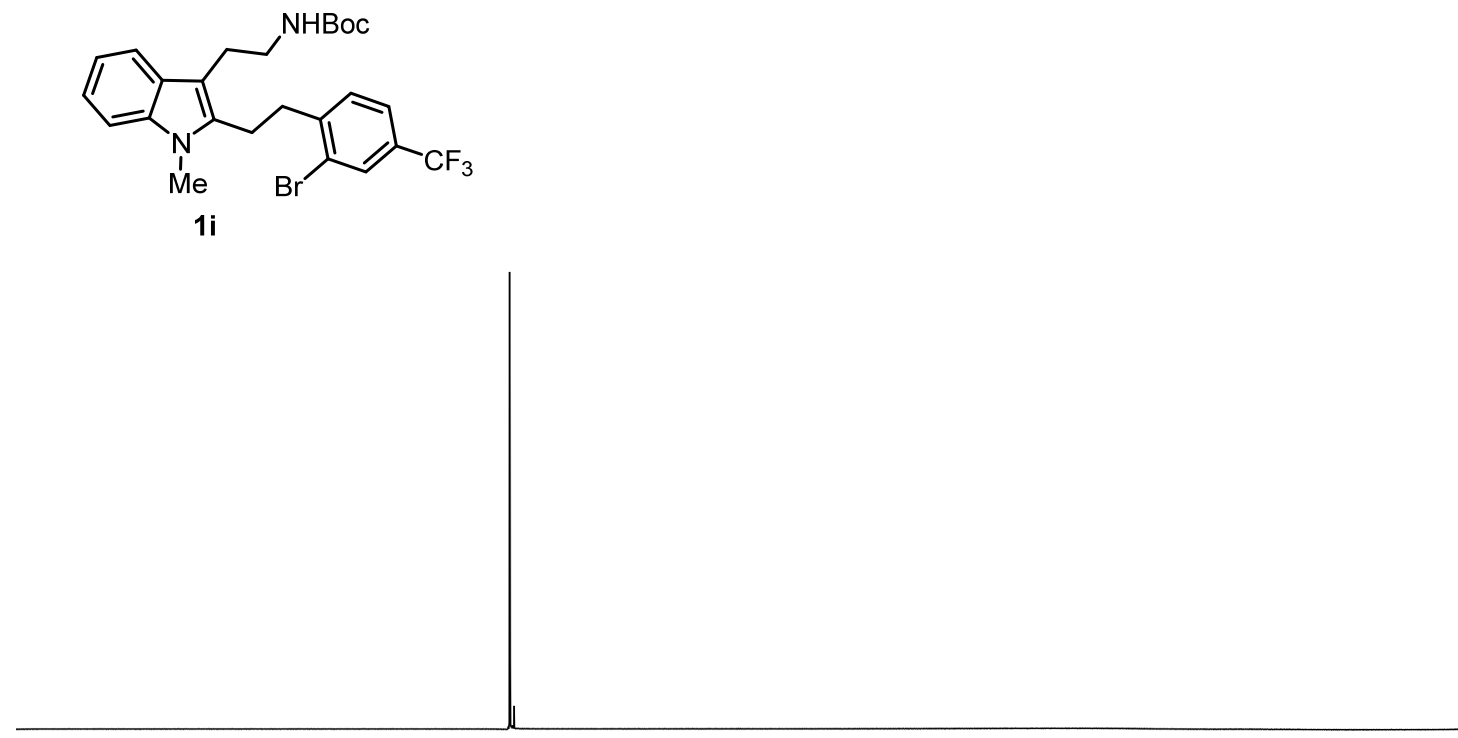

\begin{tabular}{llllllllllllllllllllllllll}
\hline 10 & 0 & -10 & -20 & -30 & -40 & -50 & -60 & -70 & -80 & -90 & -100 & -110 & -120 & -130 & -140 & -150 & -160 & -170 & -180 & -190 & -200 & -210 & 1
\end{tabular} 
${ }^{1} \mathrm{H} \mathrm{NMR}\left(\mathrm{CDCl}_{3}, 400 \mathrm{MHz}\right)$ of $\mathbf{1} \mathbf{j}$ :

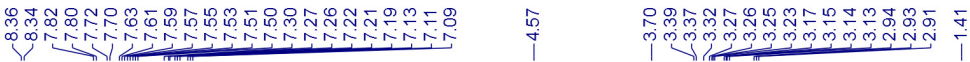

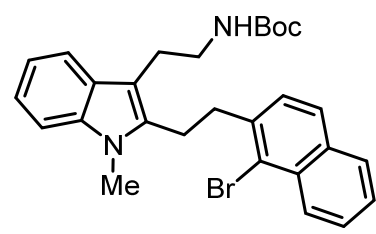

$1 \mathrm{j}$

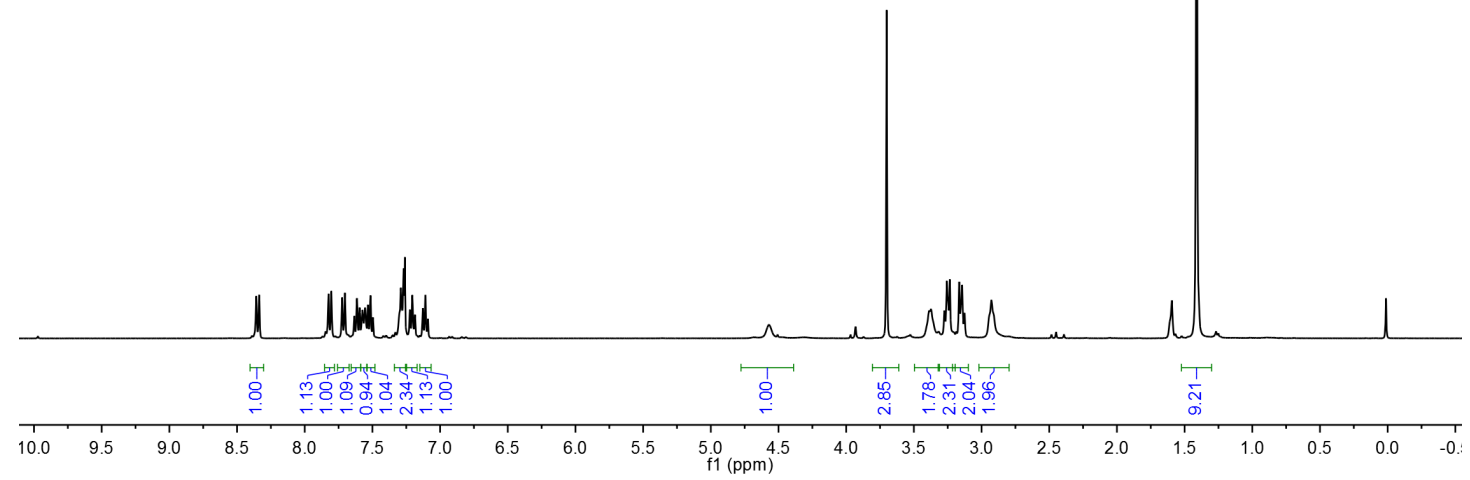

${ }^{13} \mathrm{C}\left\{{ }^{1} \mathrm{H}\right\}$ NMR $\left(\mathrm{CDCl}_{3}, 100 \mathrm{MHz}\right)$ of $\mathbf{1 j}$ :

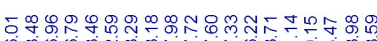

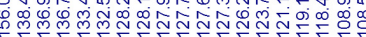

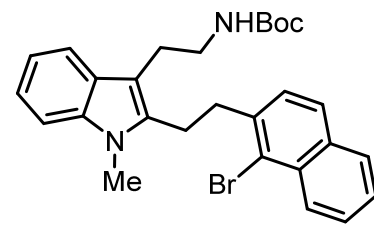

$1 \mathrm{j}$

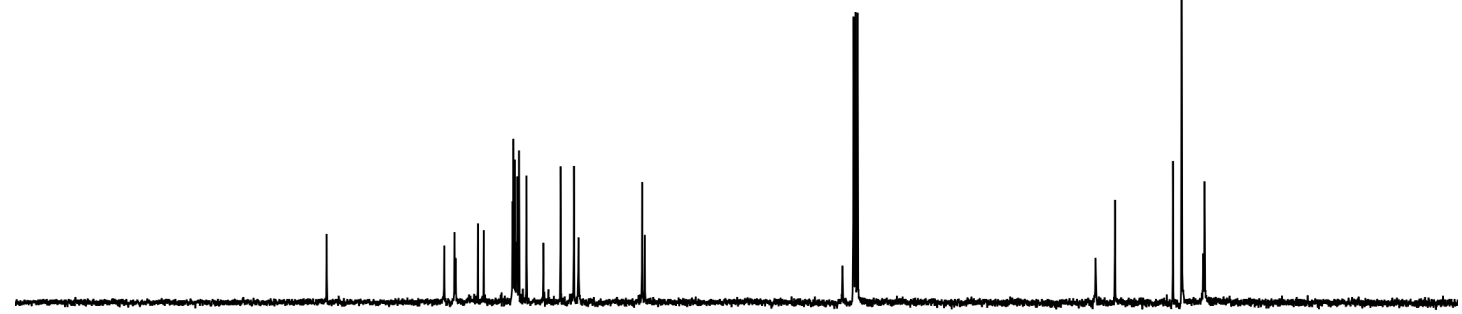

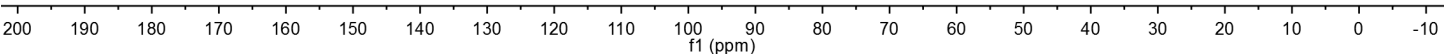


${ }^{1} \mathrm{H} \mathrm{NMR}\left(\mathrm{CDCl}_{3}, 400 \mathrm{MHz}\right)$ of $\mathbf{1 k}$ :

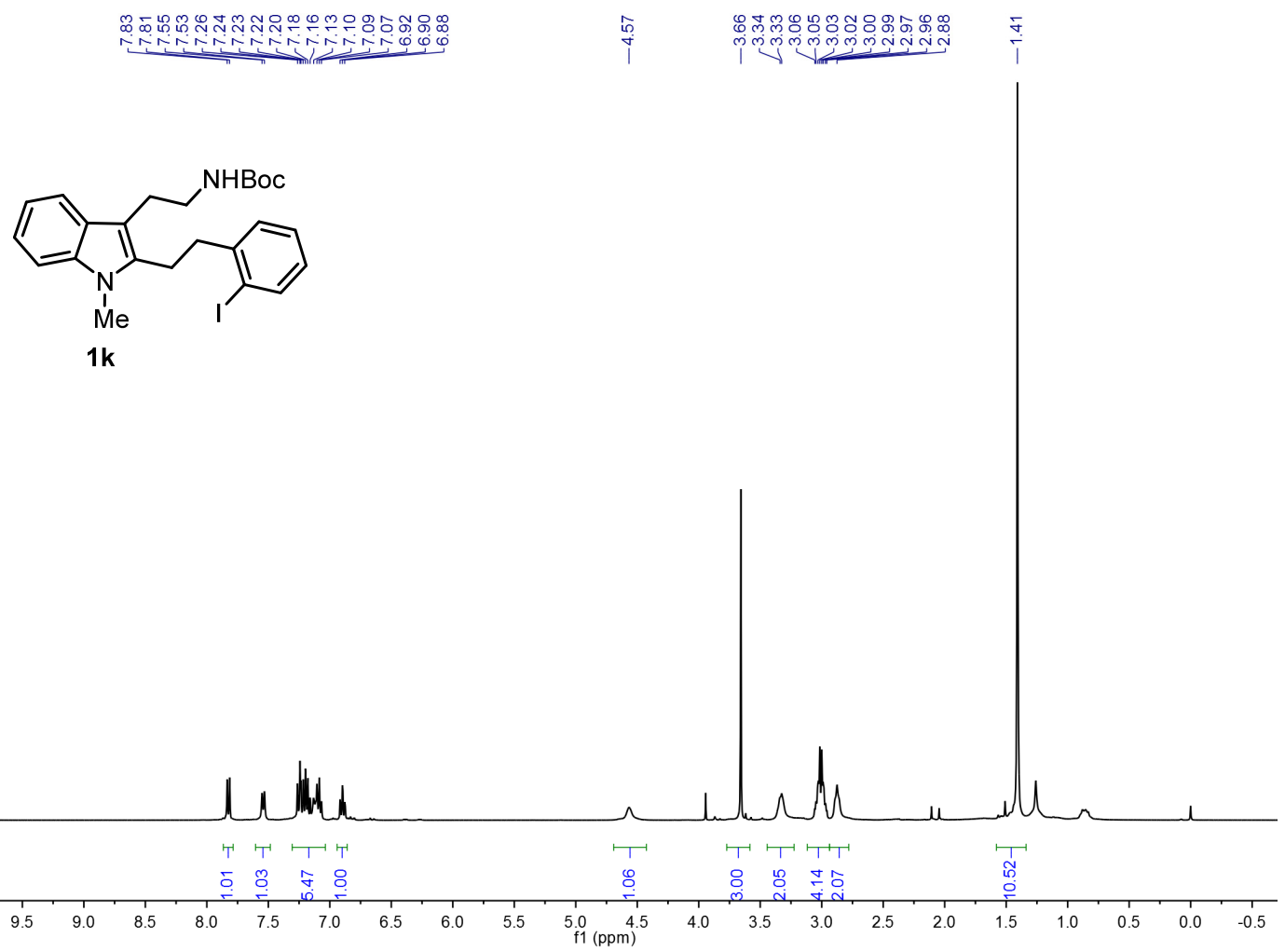

${ }^{13} \mathrm{C}\left\{{ }^{1} \mathrm{H}\right\}$ NMR $\left(\mathrm{CDCl}_{3}, 100 \mathrm{MHz}\right)$ of $\mathbf{1 k}$ :
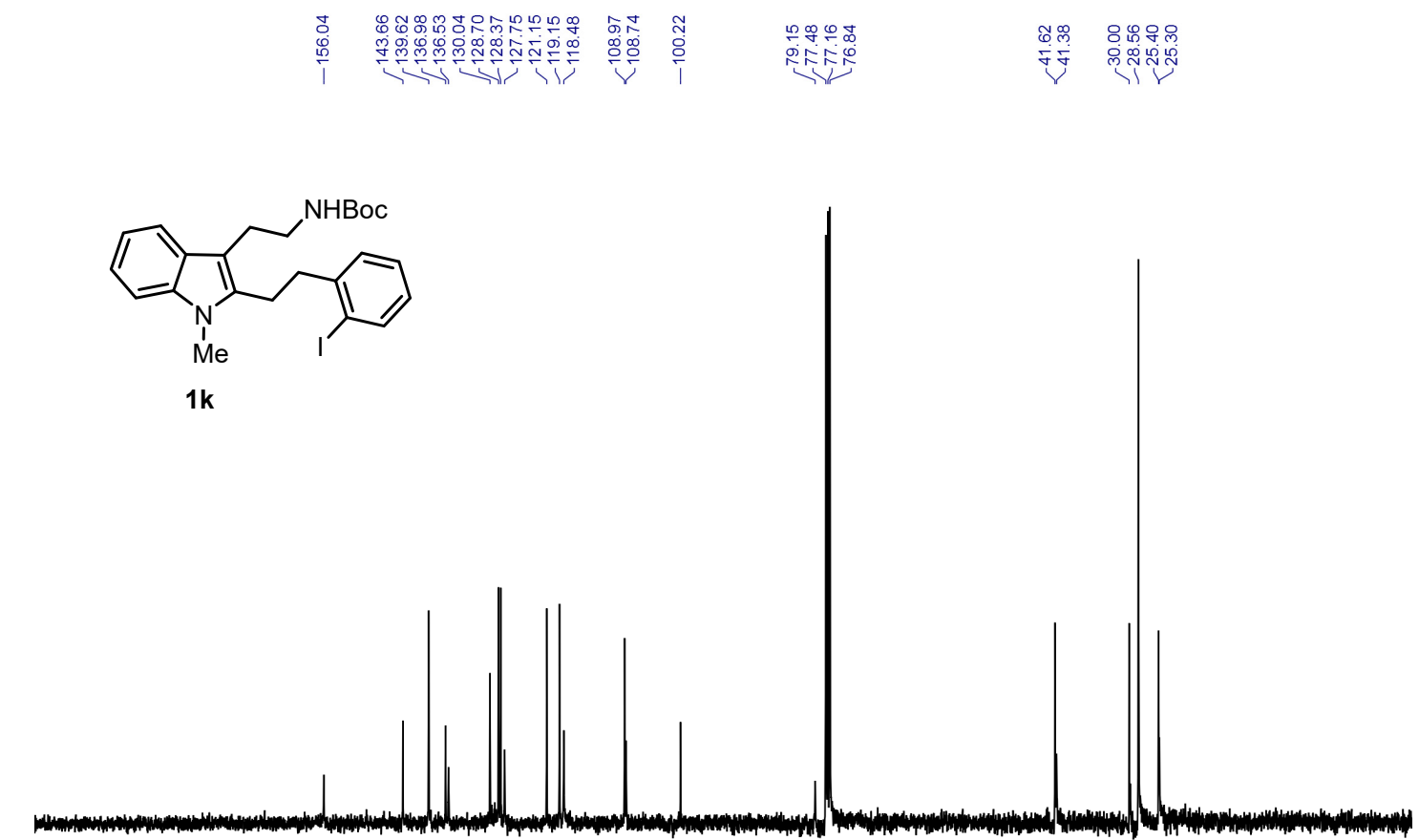

$1 \mathrm{k}$

年

ํำ

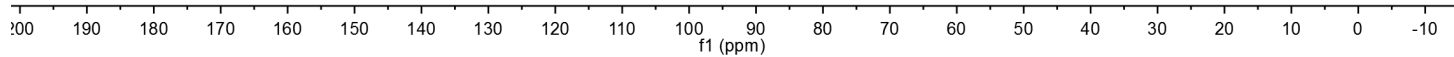


${ }^{1} \mathrm{H} \mathrm{NMR}\left(\mathrm{CDCl}_{3}, 400 \mathrm{MHz}\right)$ of $\mathbf{1 I}$ :

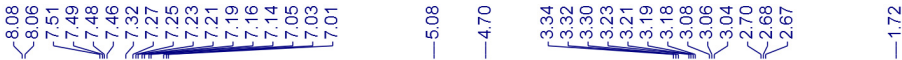

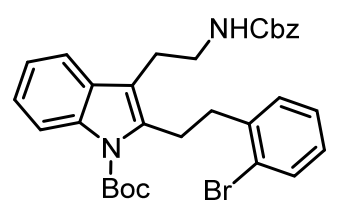

1 I

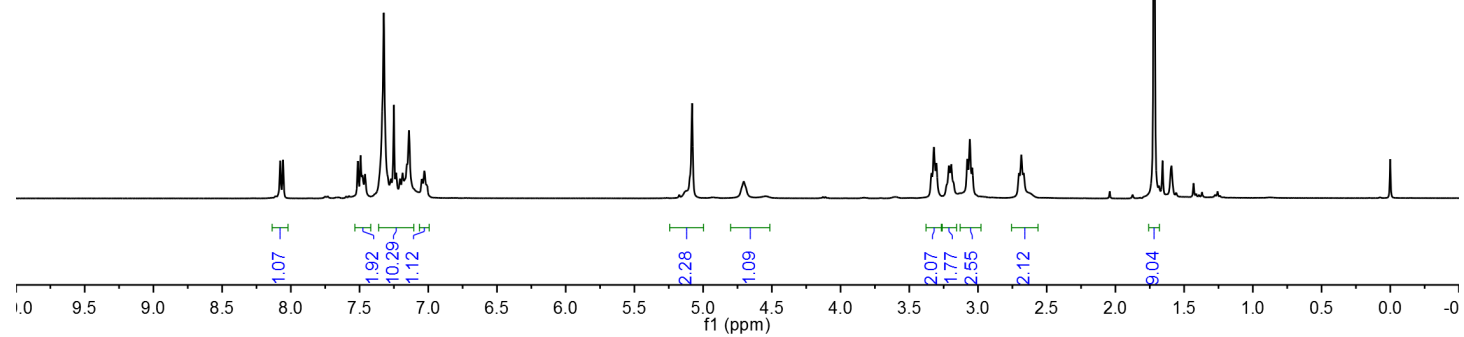

${ }^{13} \mathrm{C}\left\{{ }^{1} \mathrm{H}\right\}$ NMR $\left(\mathrm{CDCl}_{3}, 100 \mathrm{MHz}\right)$ of $\mathbf{1 I}$ :

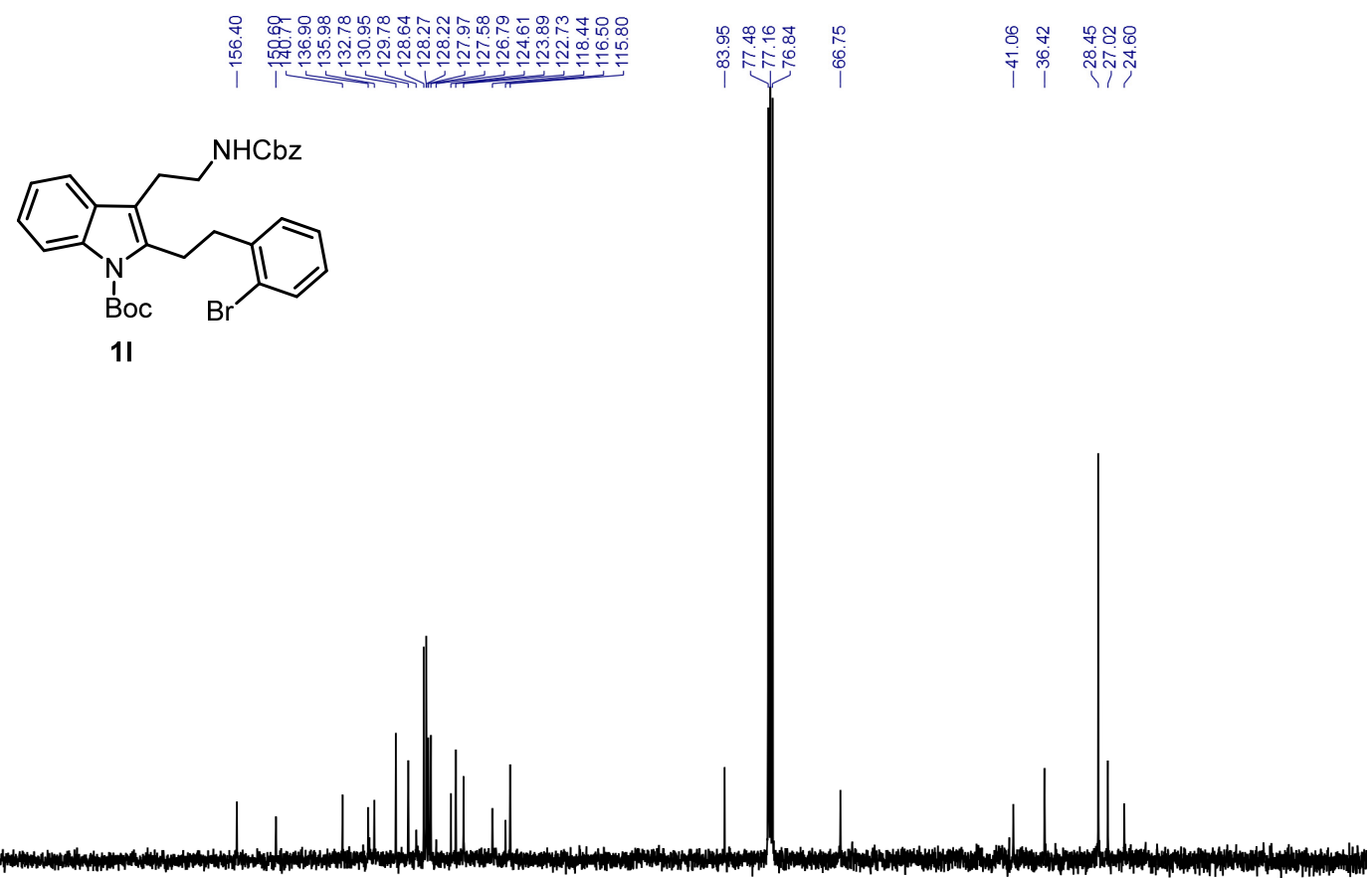

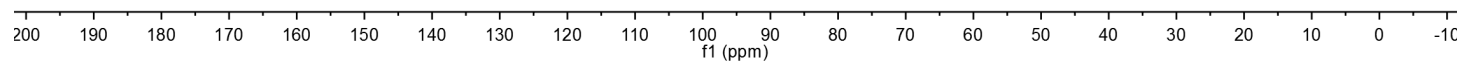


${ }^{1} \mathrm{H} \mathrm{NMR}\left(\mathrm{CDCl}_{3}, 400 \mathrm{MHz}\right)$ of $\mathbf{S 1 1}$ :

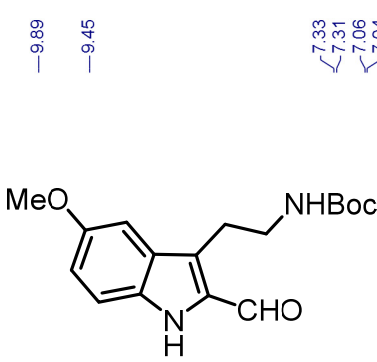

S11

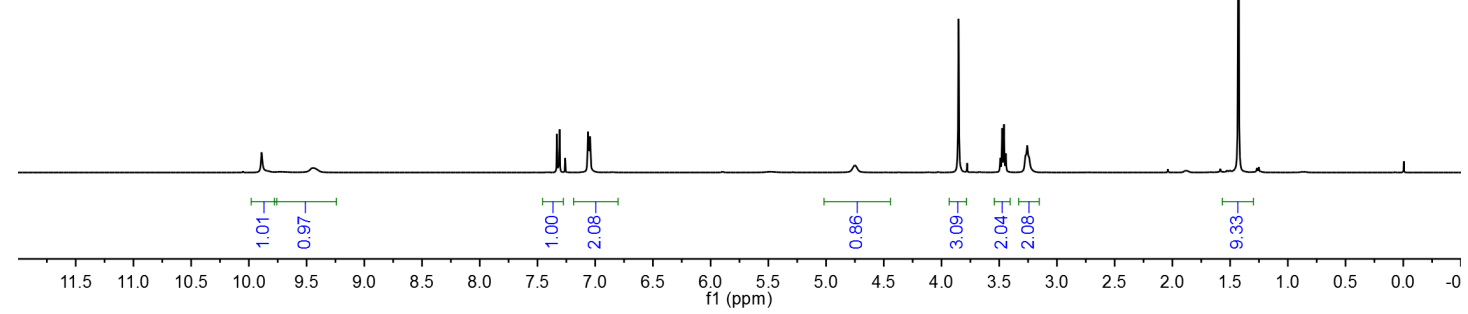

${ }^{13} \mathrm{C}\left\{{ }^{1} \mathrm{H}\right\} \mathrm{NMR}\left(\mathrm{CDCl}_{3}, 100 \mathrm{MHz}\right)$ of $\mathbf{S 1 1}$ :
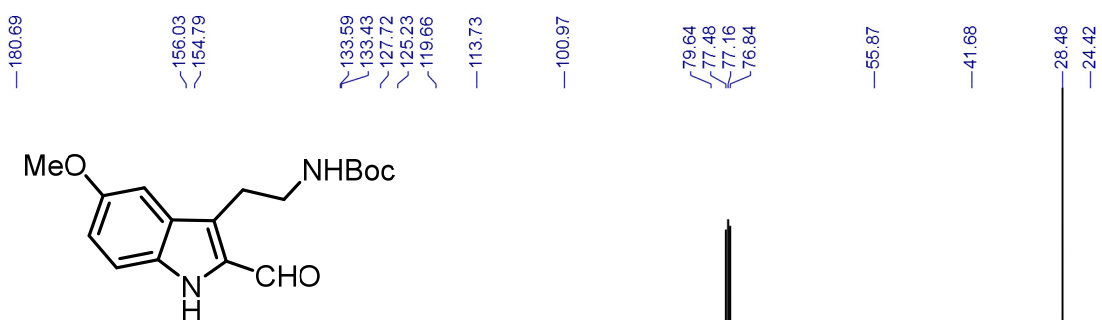

S11
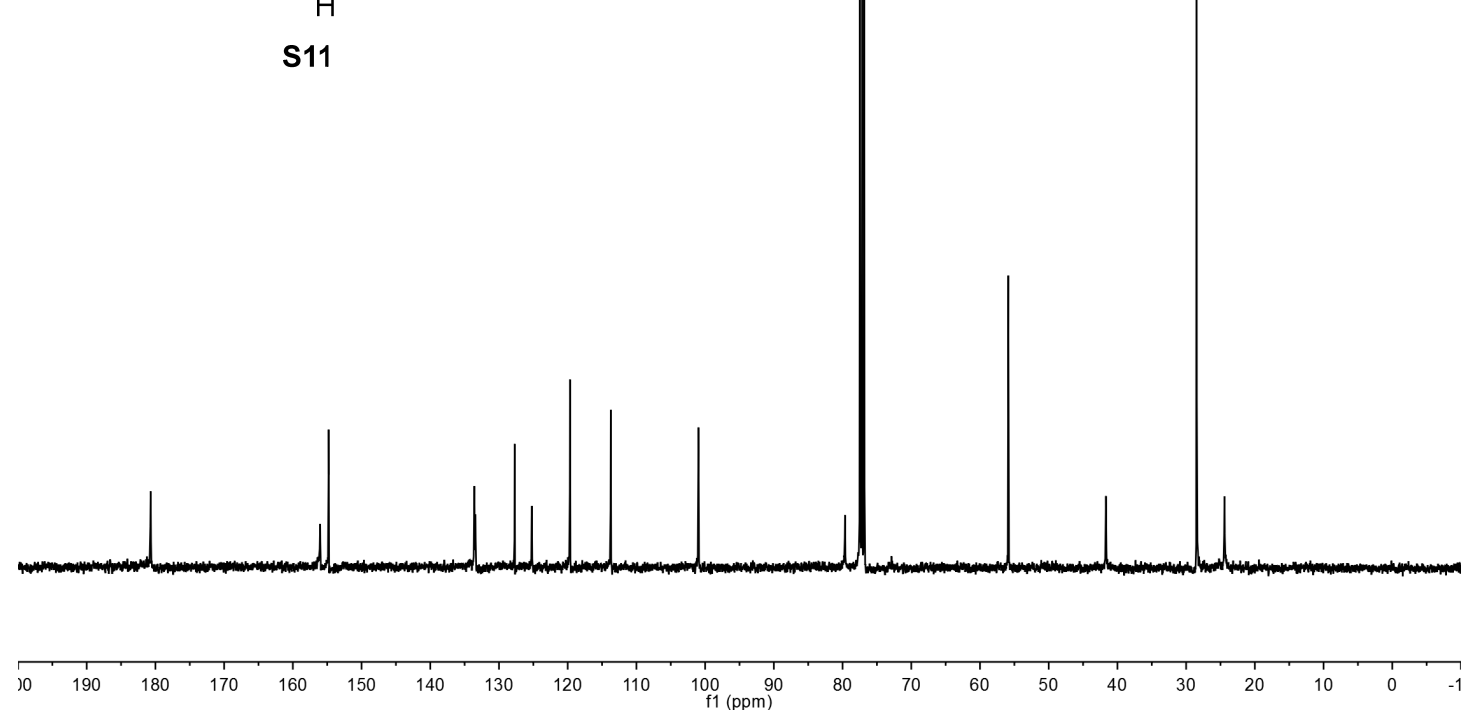
${ }^{1} \mathrm{H}$ NMR $\left(\mathrm{CDCl}_{3}, 400 \mathrm{MHz}\right)$ of $\mathbf{1 m}$ :

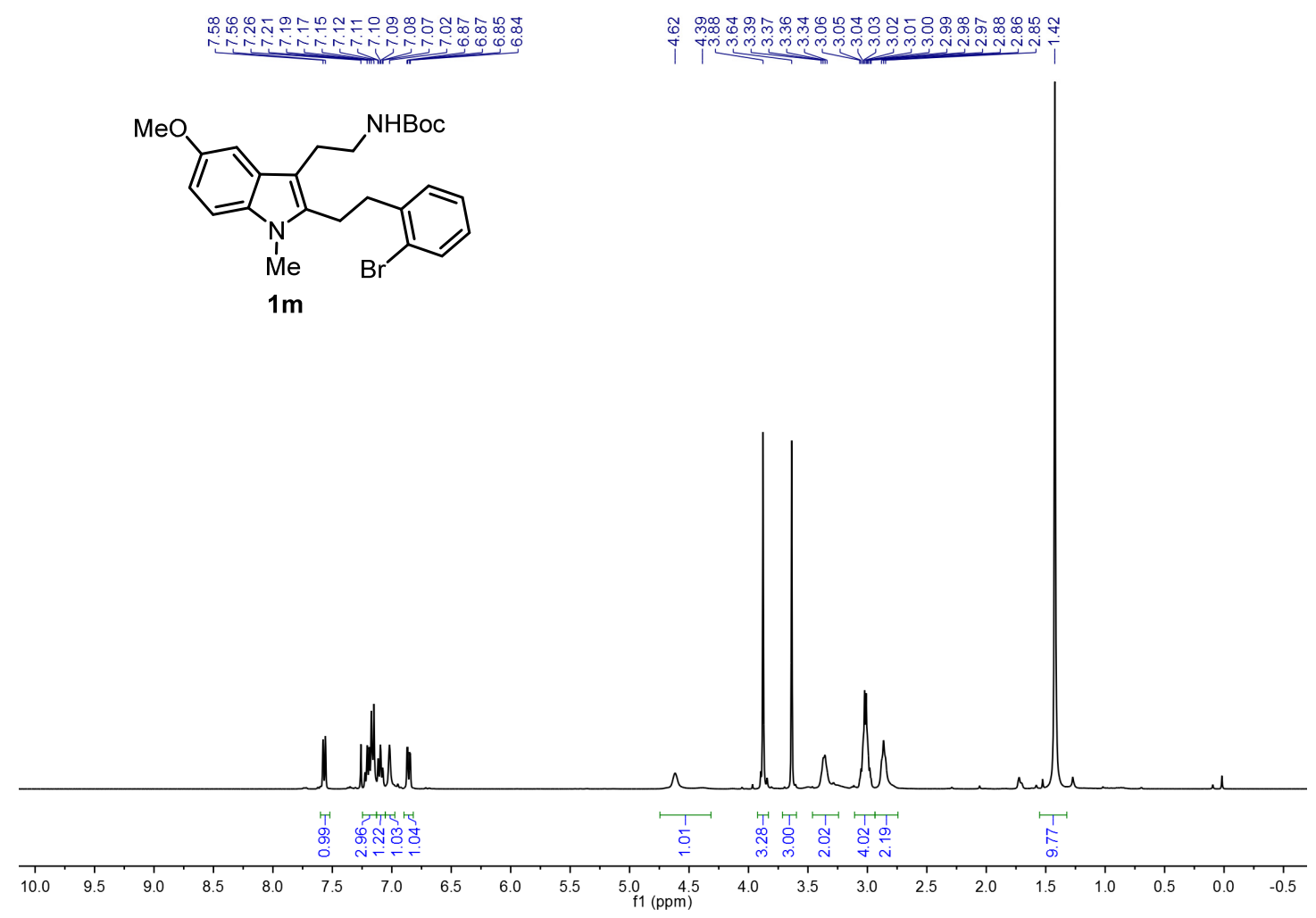

${ }^{13} \mathrm{C}\left\{{ }^{1} \mathrm{H}\right\} \mathrm{NMR}\left(\mathrm{CDCl}_{3}, 100 \mathrm{MHz}\right)$ of $\mathbf{1} \mathbf{m}:$
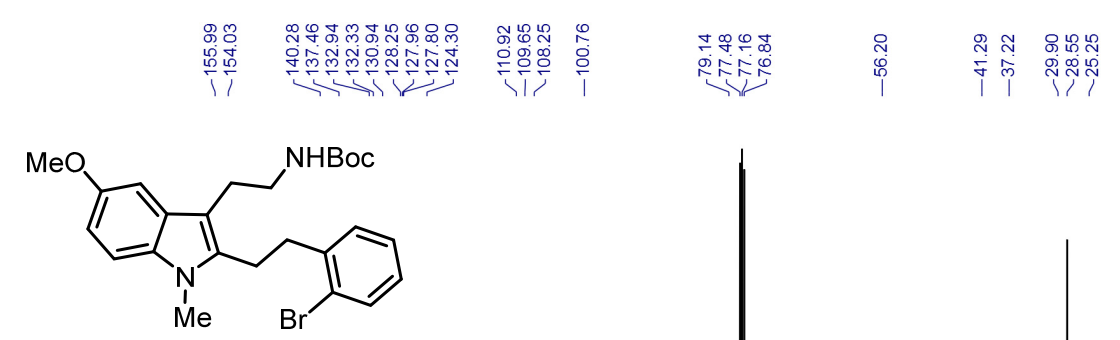

$1 \mathrm{~m}$

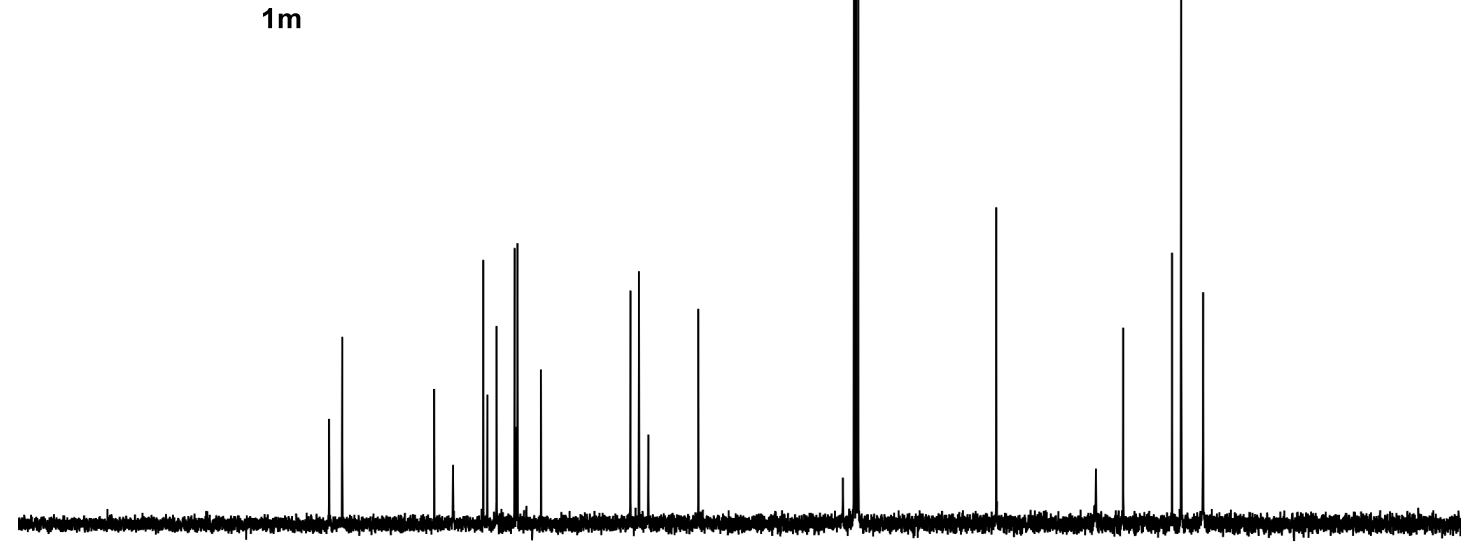

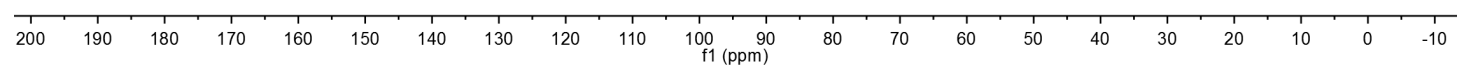


2 D HSQC NMR of $\mathbf{1 m}$ :

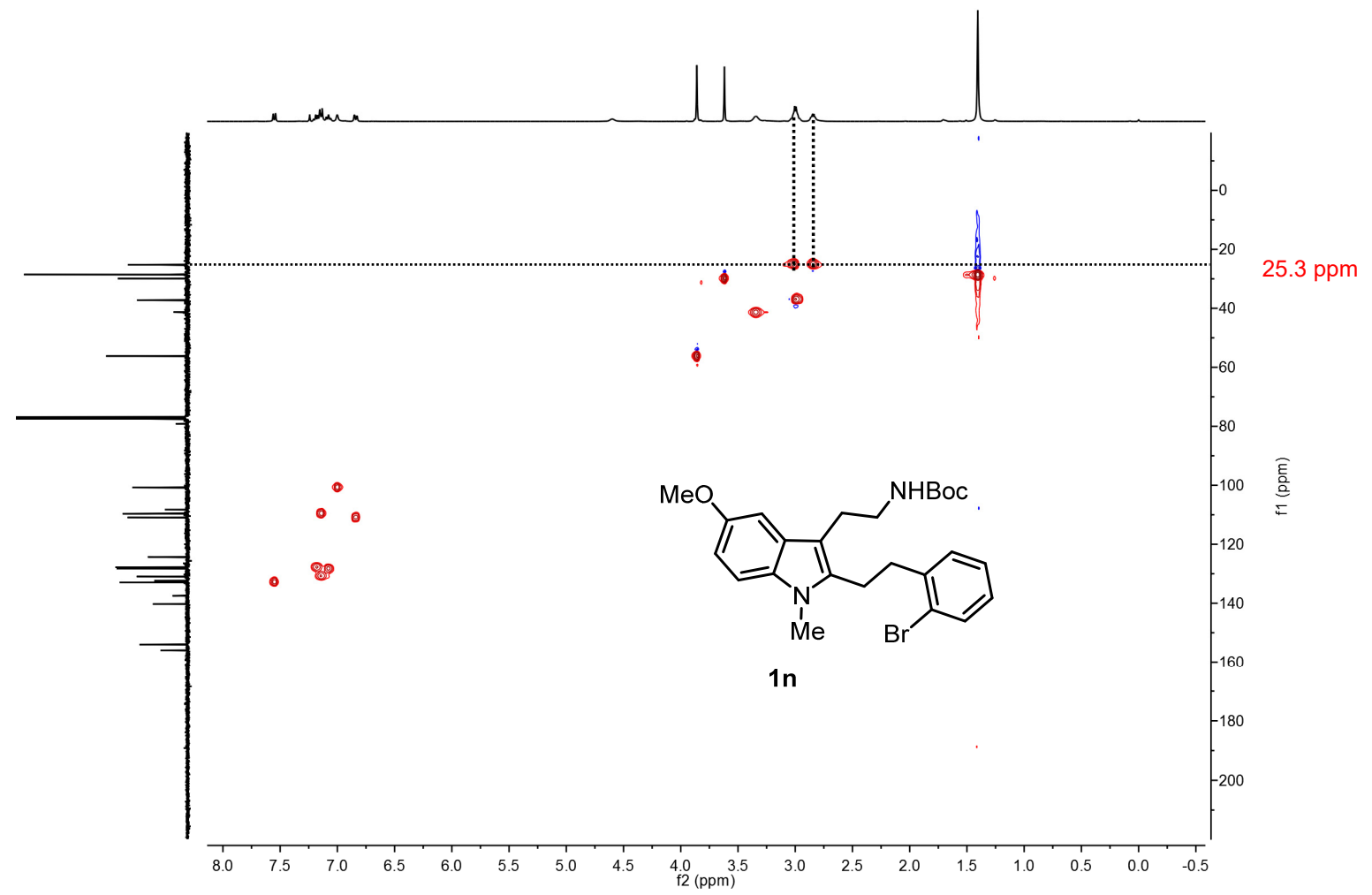


${ }^{1} \mathrm{H} \mathrm{NMR}\left(\mathrm{CDCl}_{3}, 400 \mathrm{MHz}\right)$ of $\mathbf{1 n}$ :

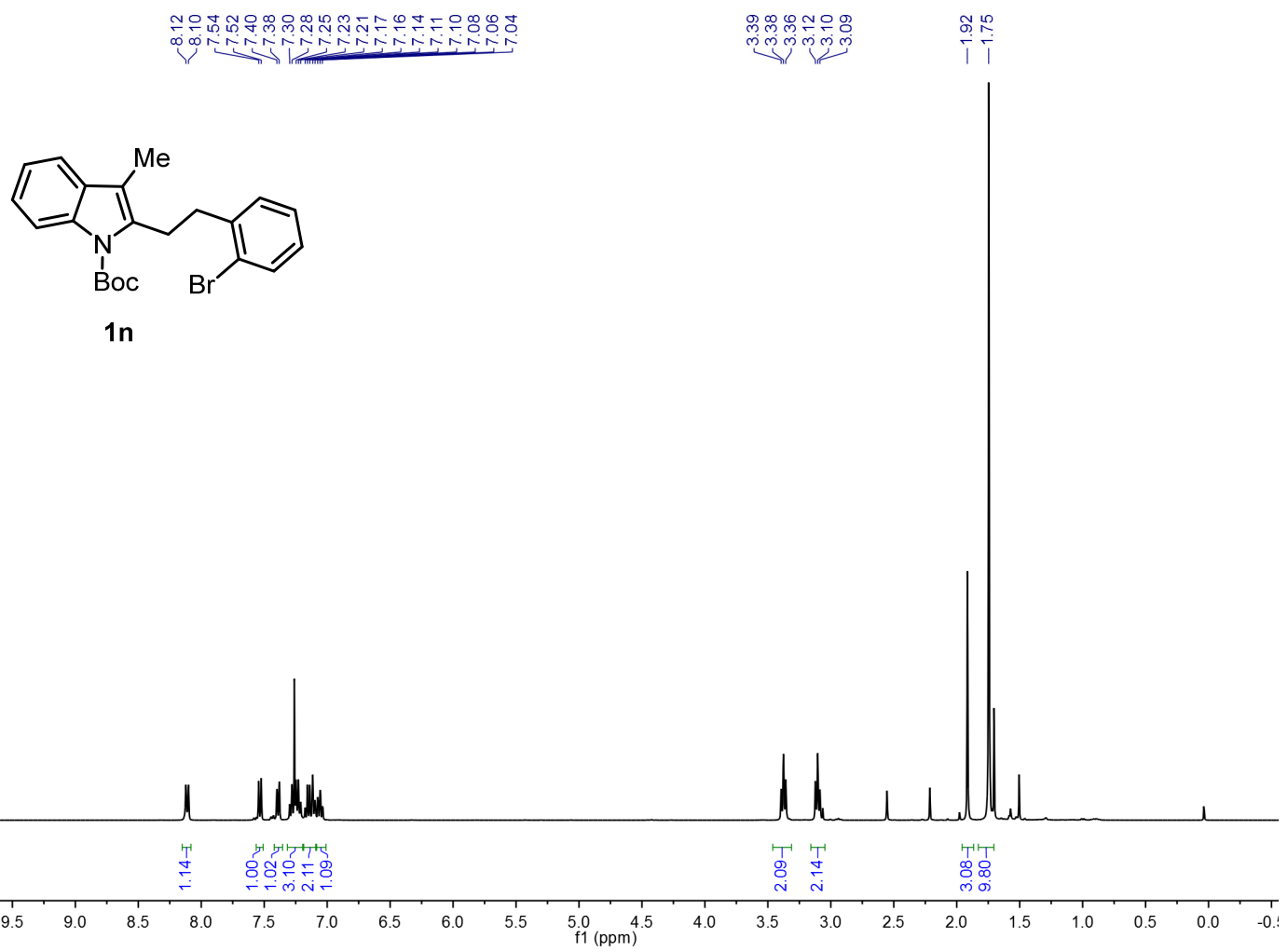

${ }^{13} \mathrm{C}\left\{{ }^{1} \mathrm{H}\right\}$ NMR $\left(\mathrm{CDCl}_{3}, 100 \mathrm{MHz}\right)$ of $\mathbf{1 n}$ :

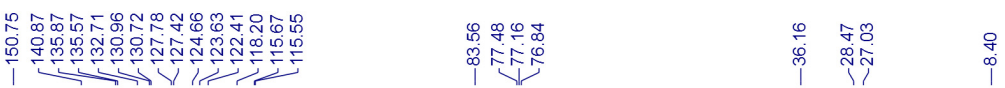<smiles>Cc1c(CCc2ccccc2Br)n(C(=O)OCc2ccccc2)c2ccccc12</smiles>

1n

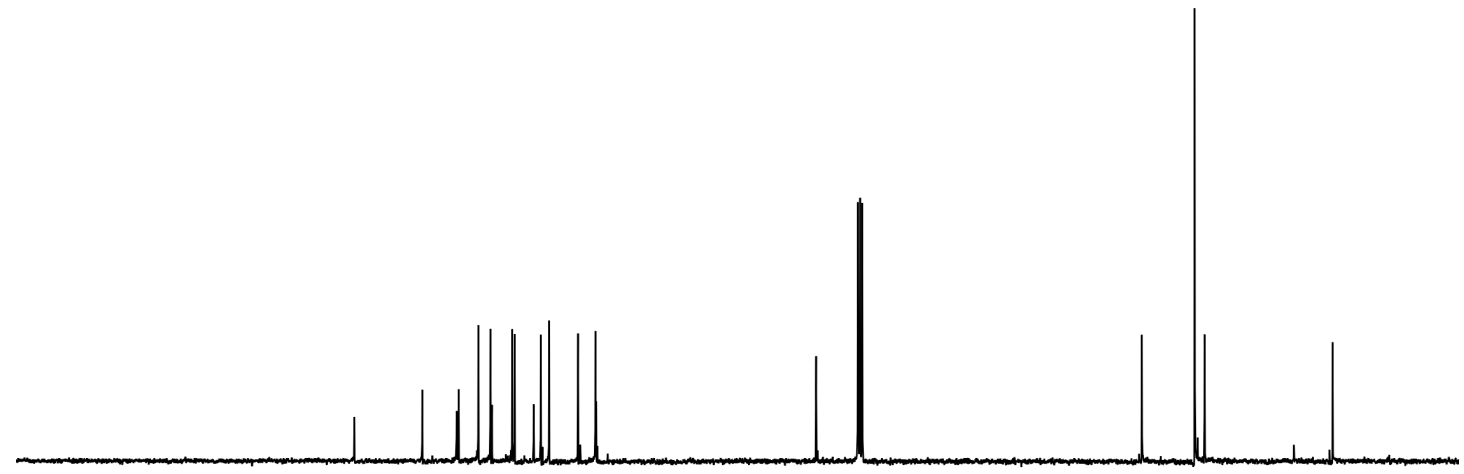

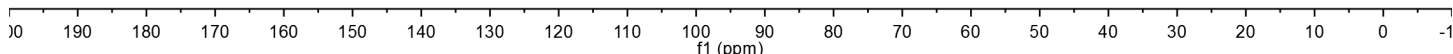


${ }^{1} \mathrm{H} \mathrm{NMR}\left(\mathrm{CDCl}_{3}, 400 \mathrm{MHz}\right)$ of $\mathbf{1 o}$ :

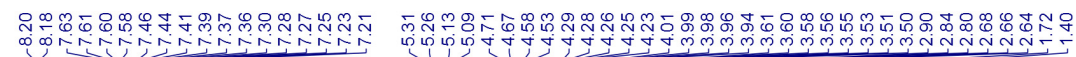<smiles>O=C(c1ccccc1Br)N1CCc2c(n(C(=O)c3ccccc3)c3ccccc23)C1</smiles>

10

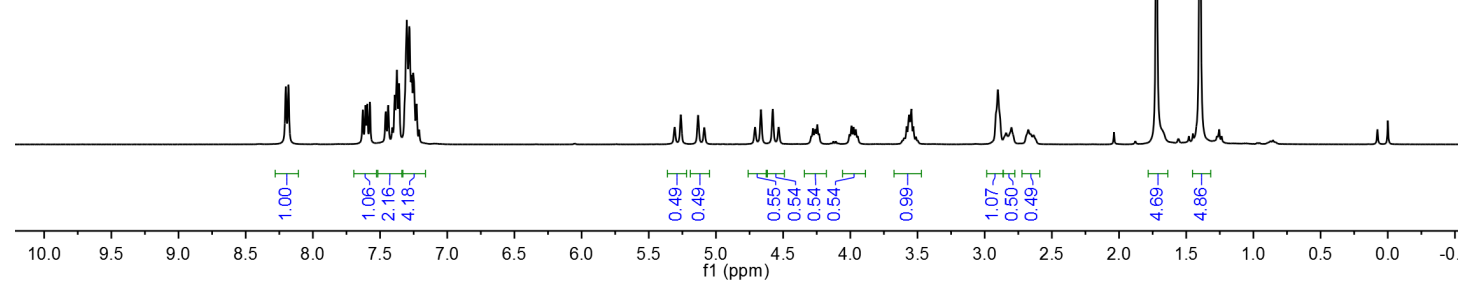

${ }^{13} \mathrm{C}\left\{{ }^{1} \mathrm{H}\right\}$ NMR $\left(\mathrm{CDCl}_{3}, 100 \mathrm{MHz}\right)$ of $\mathbf{1 0}$ :

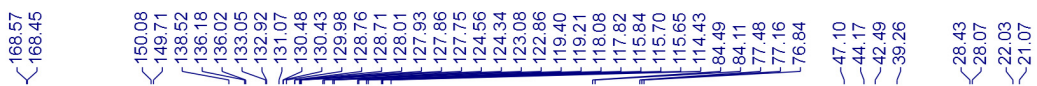

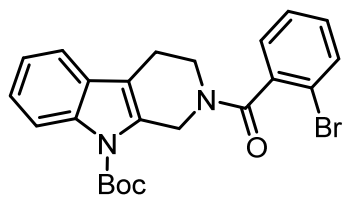

10

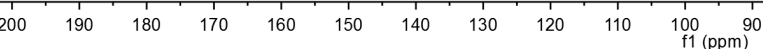


${ }^{1} \mathrm{H}$ NMR $\left(\mathrm{CDCl}_{3}, 400 \mathrm{MHz}\right)$ of 3aa:

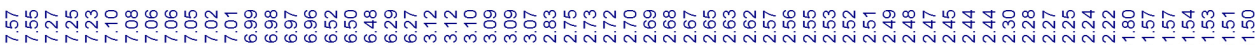

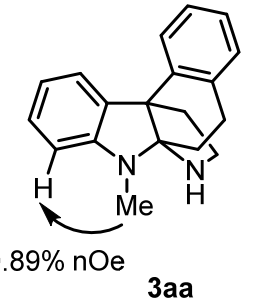

3aa

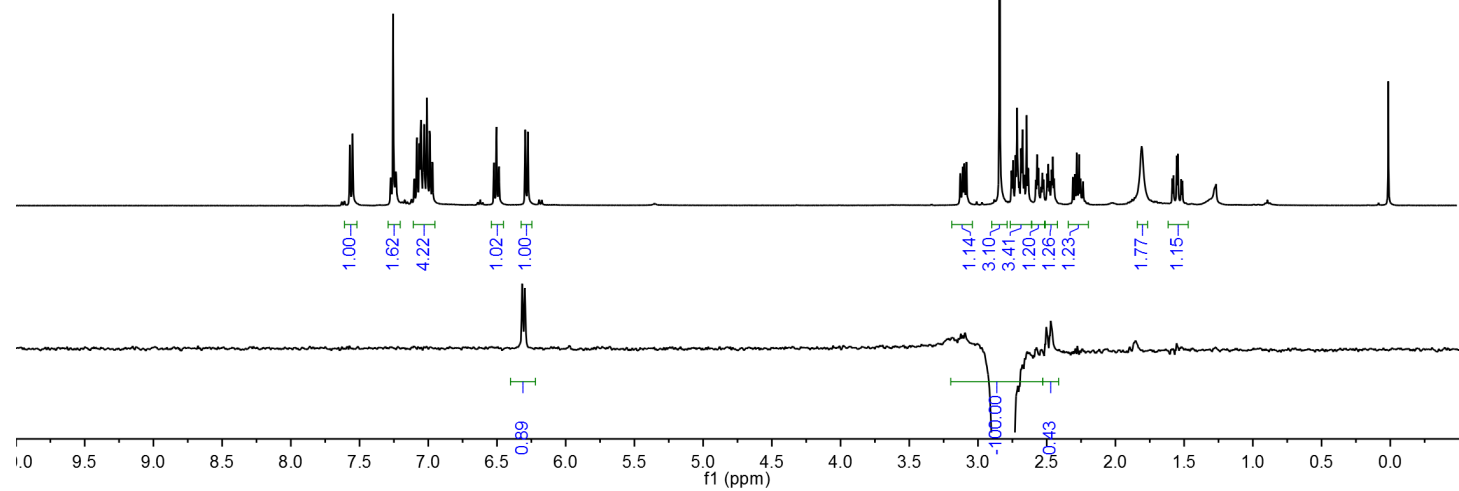

${ }^{13} \mathrm{C}\left\{{ }^{1} \mathrm{H}\right\} \mathrm{NMR}\left(\mathrm{CDCl}_{3}, 100 \mathrm{MHz}\right)$ of 3aa:

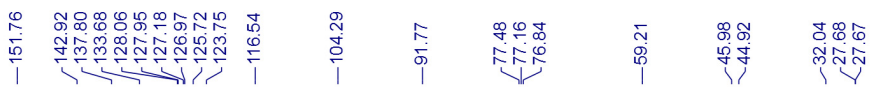

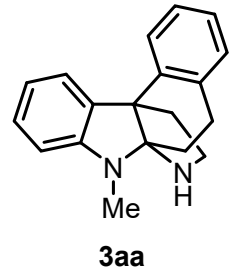

3aa

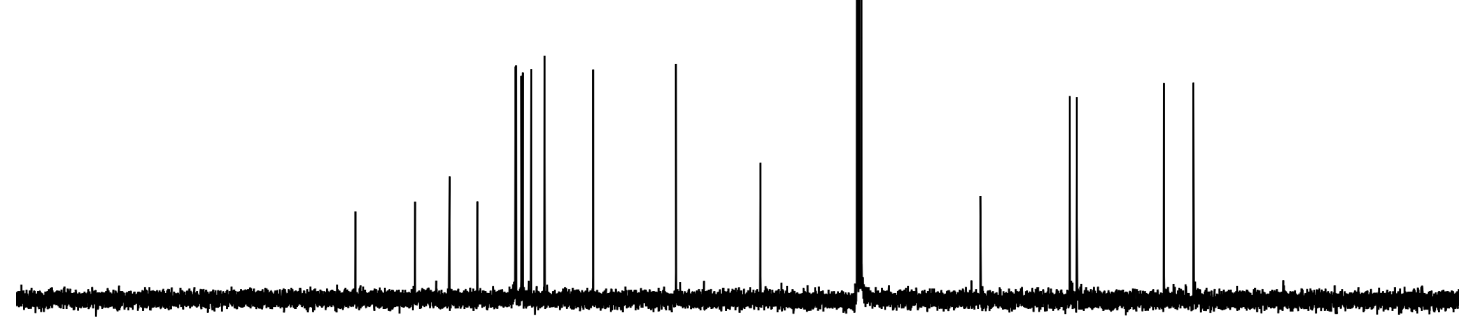

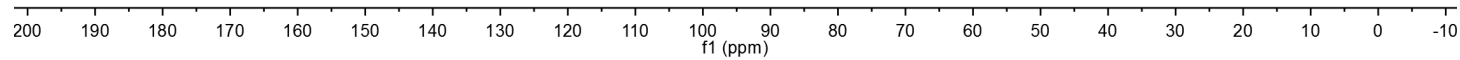


${ }^{1} \mathrm{H}$ NMR $\left(\mathrm{CDCl}_{3}, 400 \mathrm{MHz}\right)$ of 3ab:

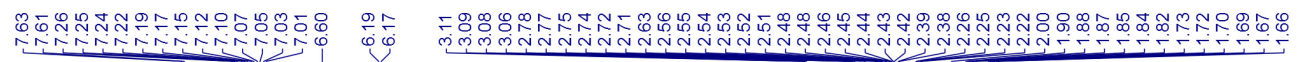

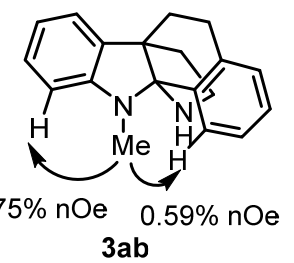

$0.75 \% \mathrm{nOe} \quad 0.59 \% \mathrm{nOe}$
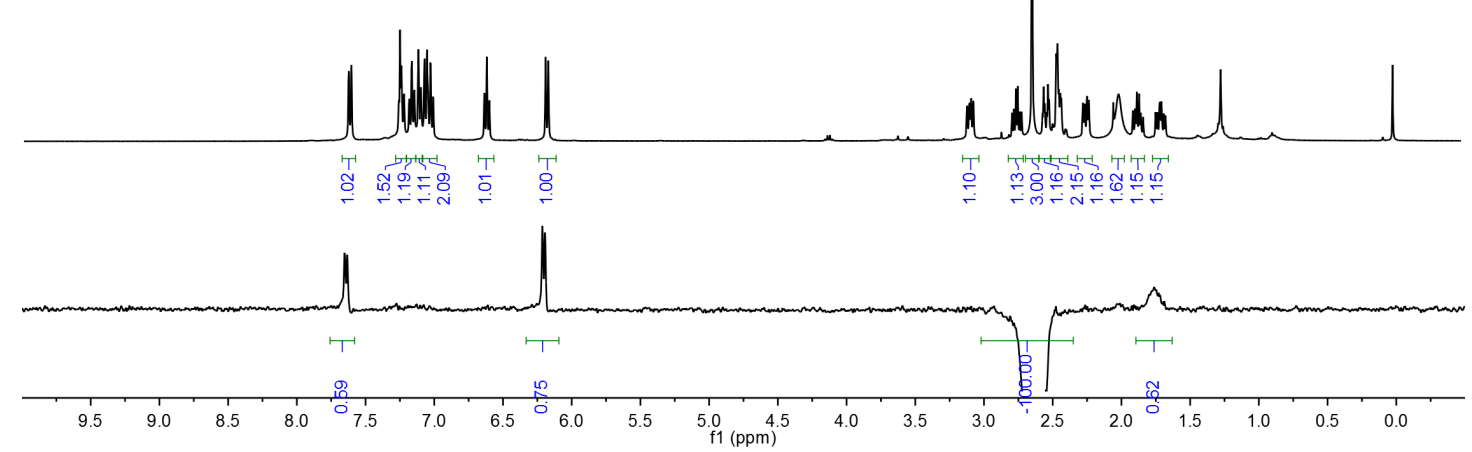

${ }^{13} \mathrm{C}\left\{{ }^{1} \mathrm{H}\right\}$ NMR $\left(\mathrm{CDCl}_{3}, 100 \mathrm{MHz}\right)$ of $\mathbf{3 a b}$ :

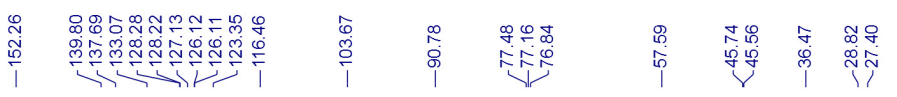

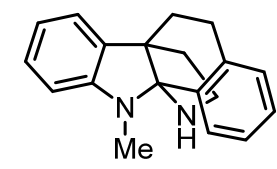

$3 a b$

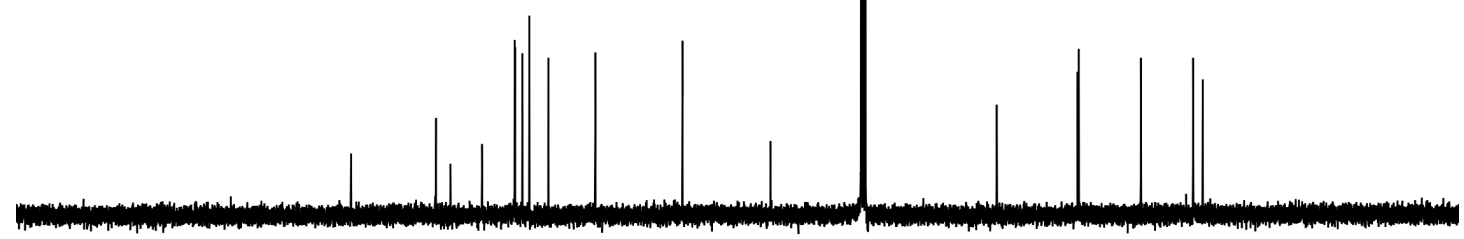

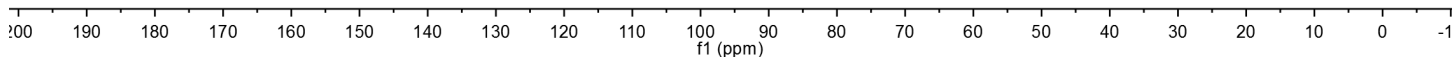


${ }^{1} \mathrm{H} \mathrm{NMR}\left(\mathrm{CDCl}_{3}, 400 \mathrm{MHz}\right)$ of $\mathbf{3 b a}$ :

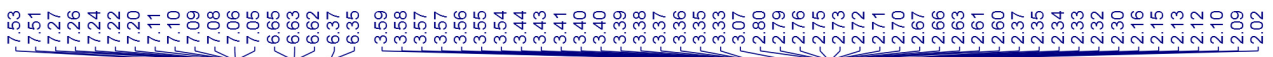

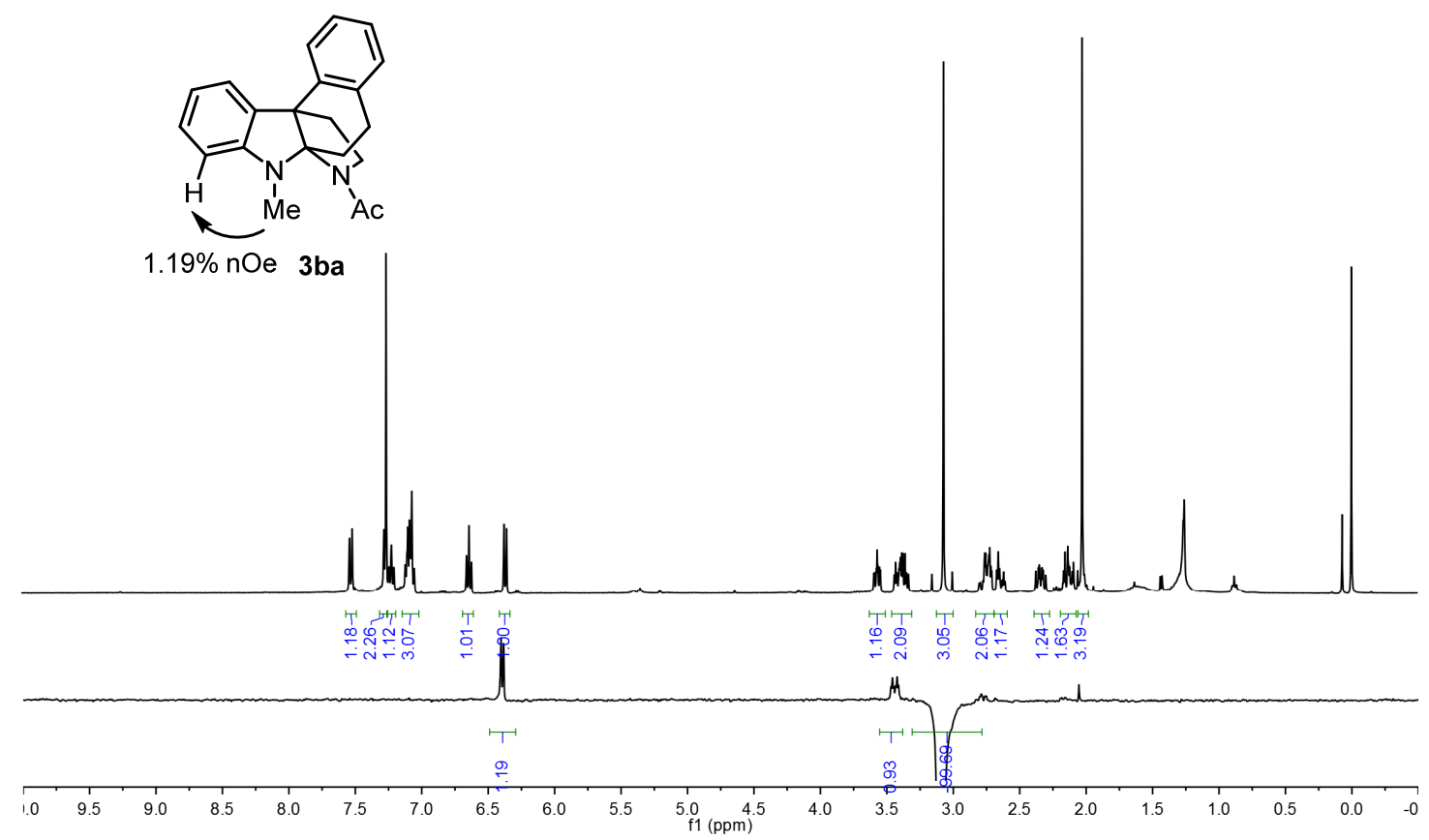

${ }^{13} \mathrm{C}\left\{{ }^{1} \mathrm{H}\right\}$ NMR $\left(\mathrm{CDCl}_{3}, 100 \mathrm{MHz}\right)$ of $\mathbf{3 b a}:$

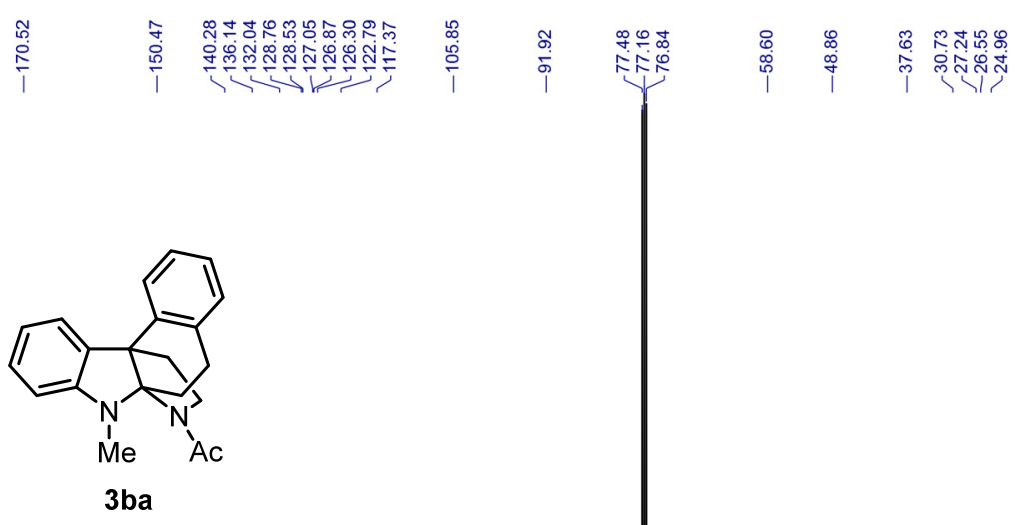

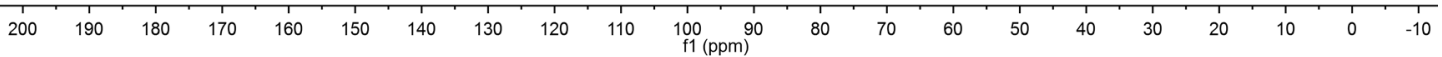


${ }^{1} \mathrm{H} \mathrm{NMR}\left(\mathrm{CDCl}_{3}, 400 \mathrm{MHz}\right)$ of 3da:

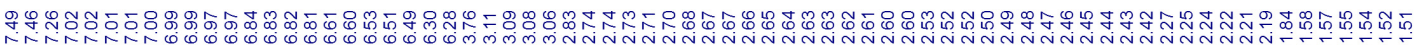<smiles>COc1ccc2c(c1)CCNC21NNCCO1</smiles>

$1.13 \% \mathrm{nOe} \quad 3 \mathrm{da}$

${ }^{13} \mathrm{C}\left\{{ }^{1} \mathrm{H}\right\}$ NMR $\left(\mathrm{CDCl}_{3}, 100 \mathrm{MHz}\right)$ of $\mathbf{3 d a}:$

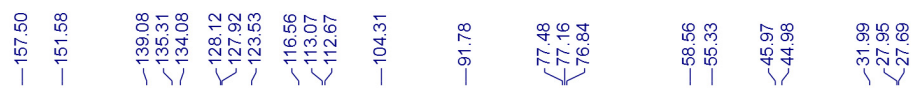<smiles>COc1ccc2c(c1)CCNC21CCCC1</smiles>

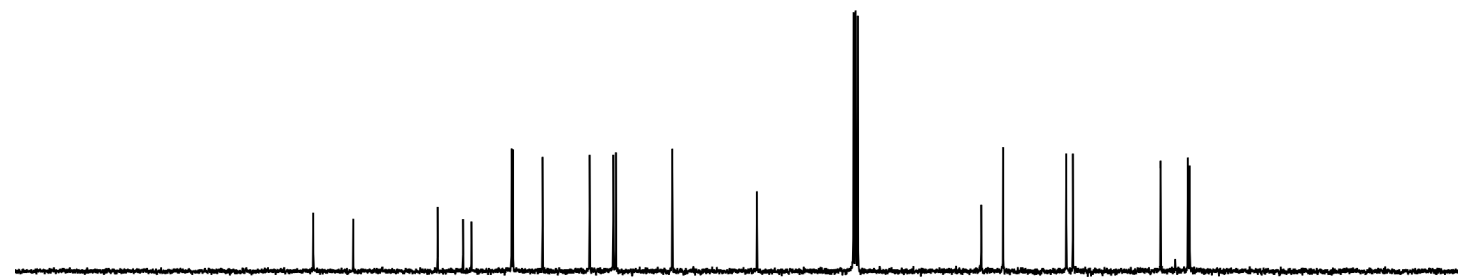

\begin{tabular}{lllllllllllllllllllll}
\hline & 1 \\
200 & 190 & 180 & 170 & 160 & 150 & 140 & 130 & 120 & 110 & $\underset{\mathrm{f} 1(\mathrm{ppm})}{100}$ & 80 & 70 & 60 & 50 & 40 & 30 & 20 & 10 & 0 & -10
\end{tabular} 
${ }^{1} \mathrm{H} \mathrm{NMR}\left(\mathrm{CDCl}_{3}, 400 \mathrm{MHz}\right)$ of 3ea:

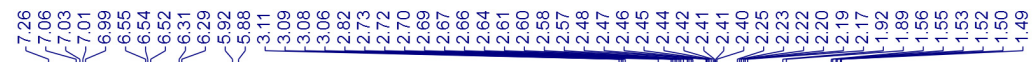

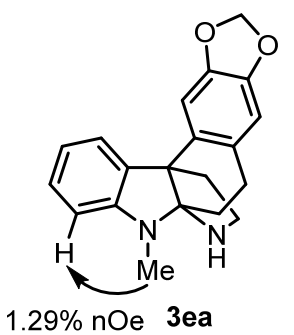

huldhuld

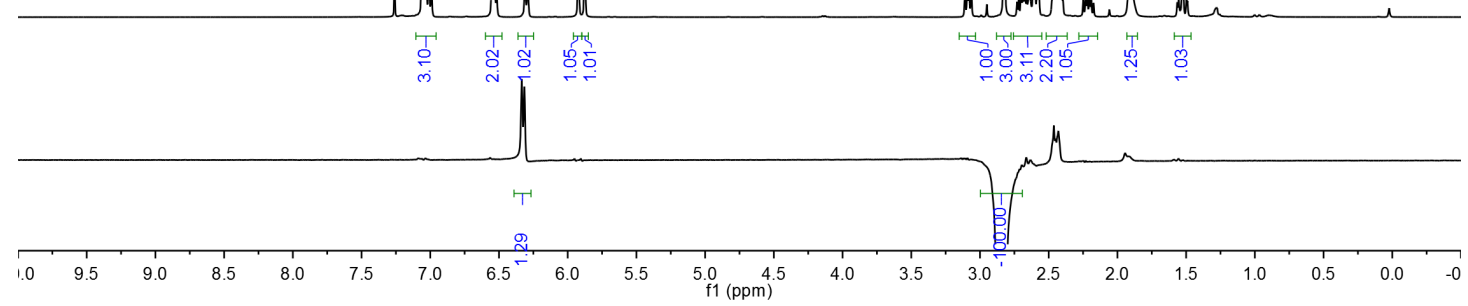

${ }^{13} \mathrm{C}\left\{{ }^{1} \mathrm{H}\right\}$ NMR $\left(\mathrm{CDCl}_{3}, 100 \mathrm{MHz}\right)$ of $\mathbf{3 e a}:$
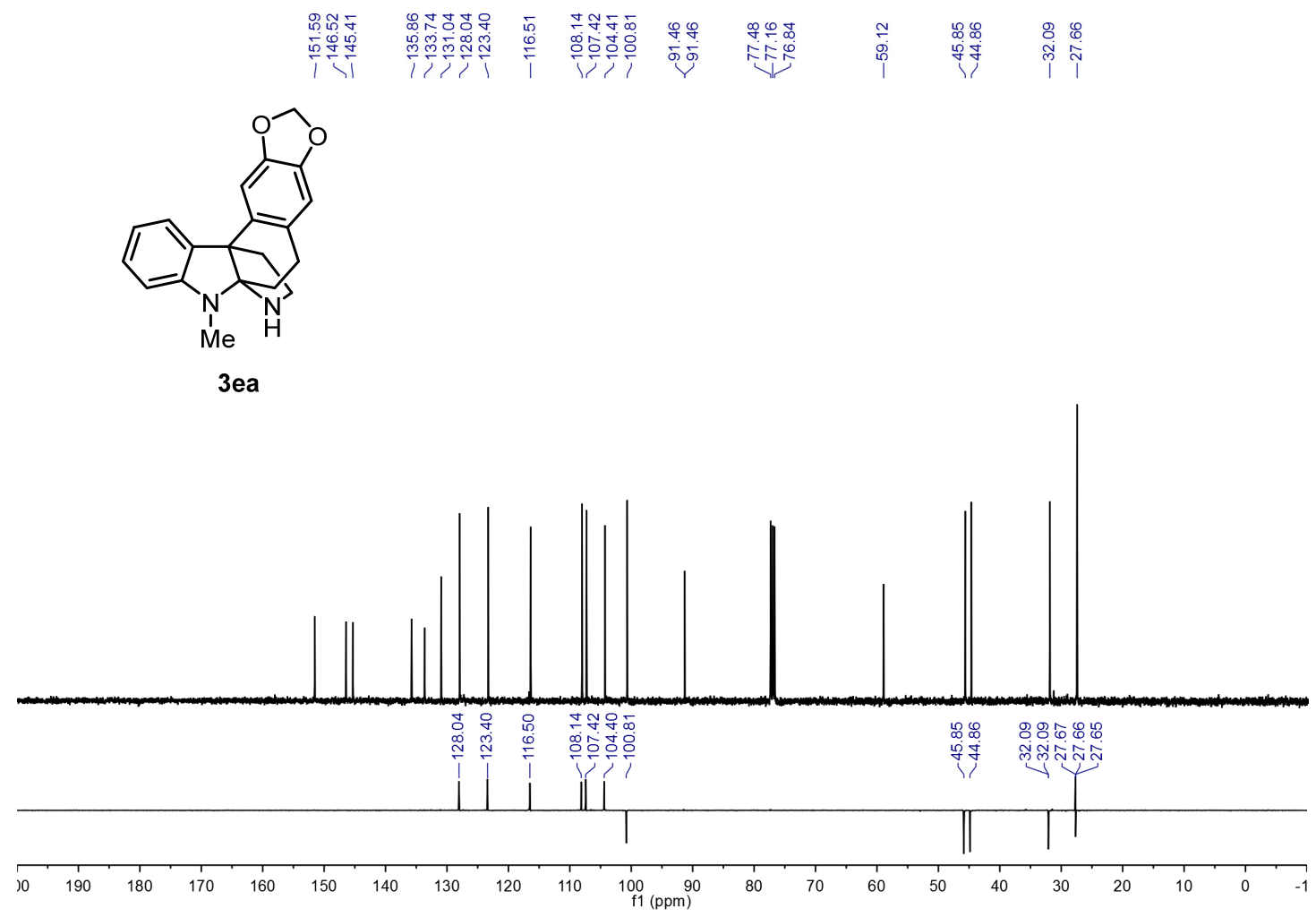
${ }^{1} \mathrm{H} \mathrm{NMR}\left(\mathrm{CDCl}_{3}, 400 \mathrm{MHz}\right)$ of $\mathbf{3 e b}$ :

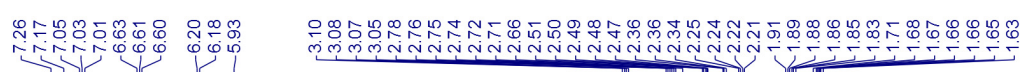

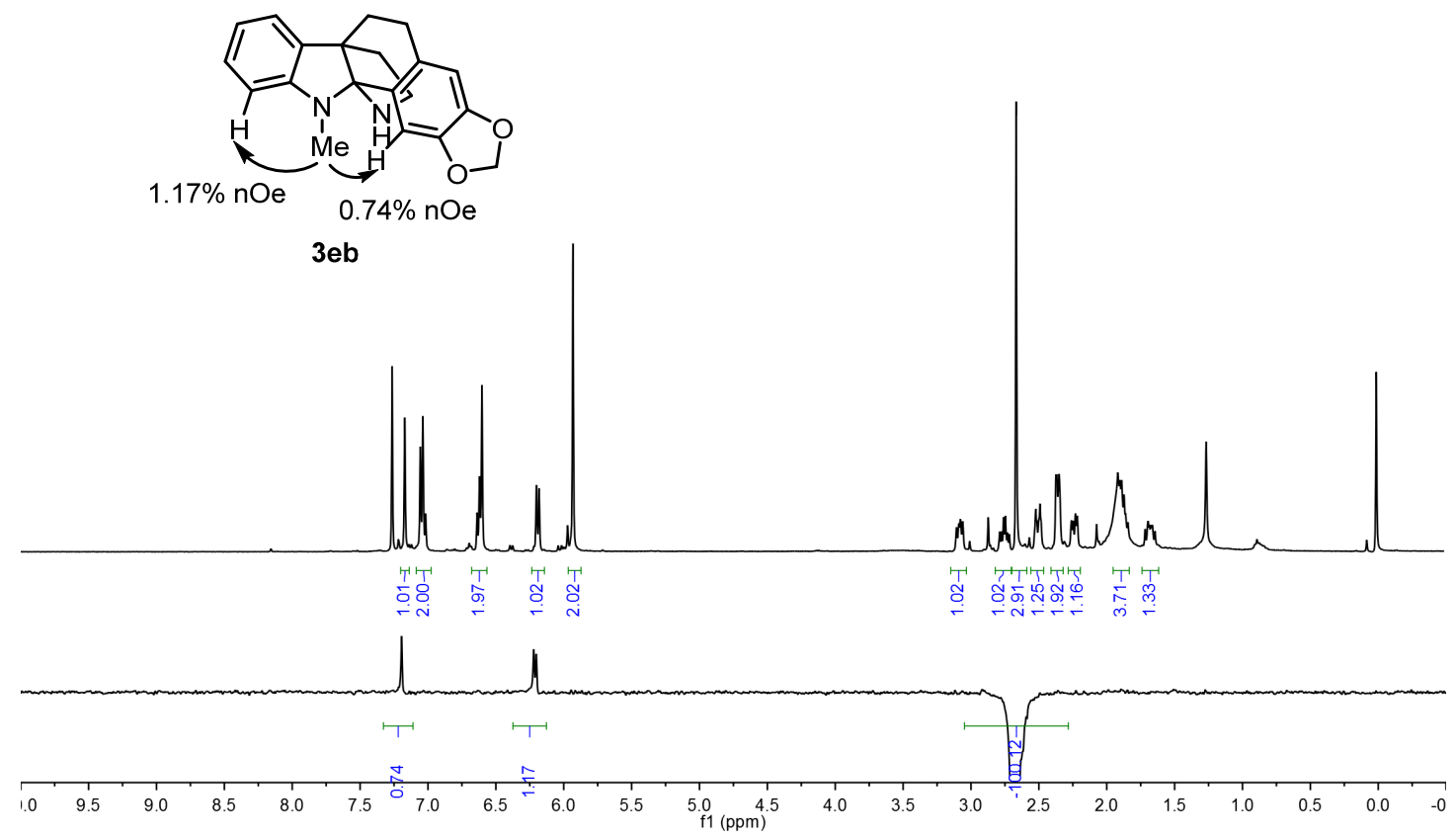

${ }^{13} \mathrm{C}\left\{{ }^{1} \mathrm{H}\right\}$ NMR $\left(\mathrm{CDCl}_{3}, 100 \mathrm{MHz}\right)$ of $\mathbf{3 e b}$ :

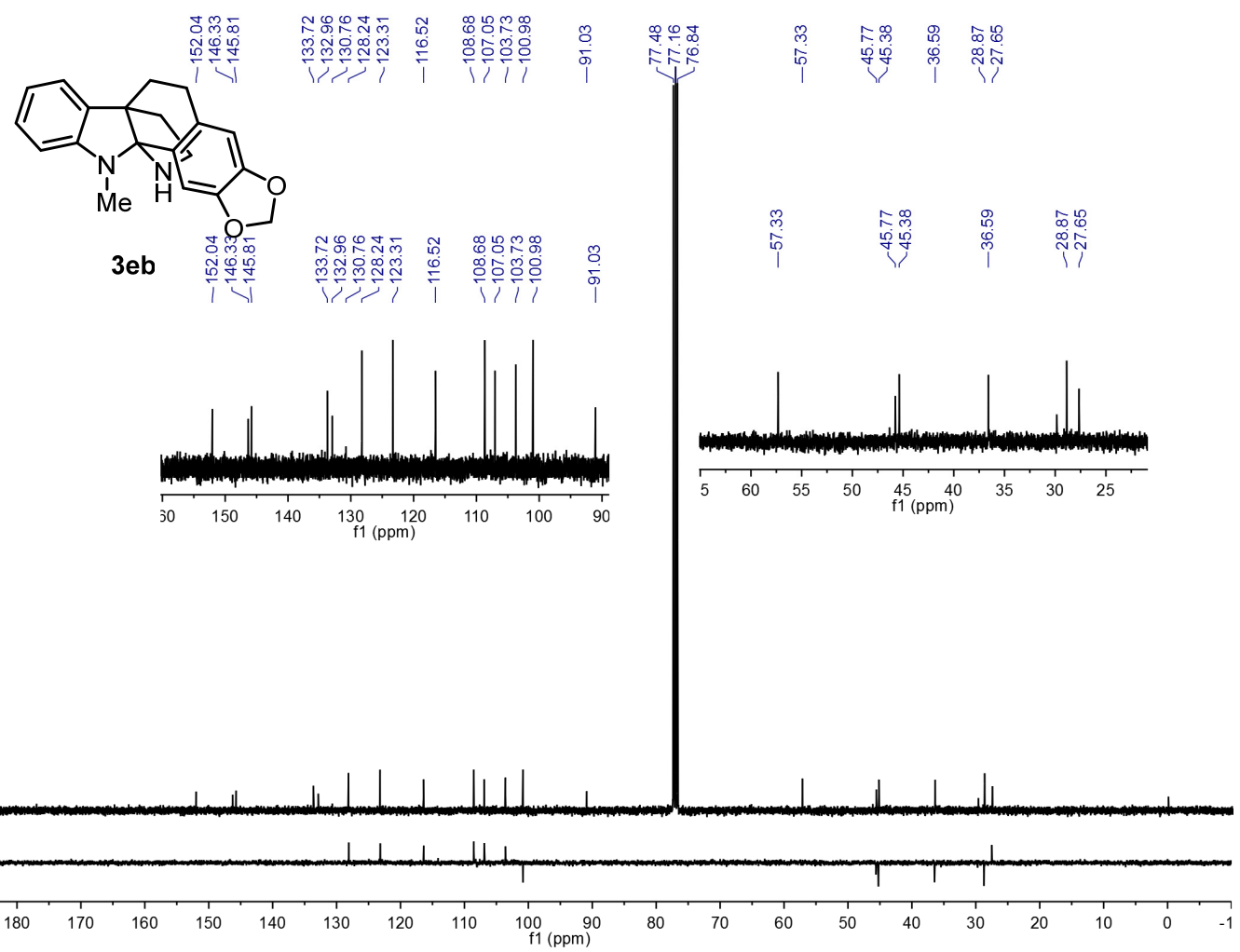


${ }^{1} \mathrm{H}$ NMR $\left(\mathrm{CDCl}_{3}, 400 \mathrm{MHz}\right)$ of $\mathbf{3 f a}$ :

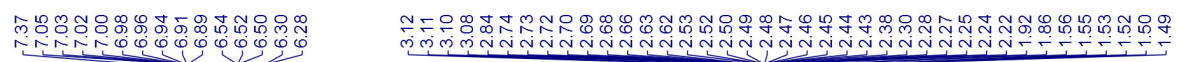

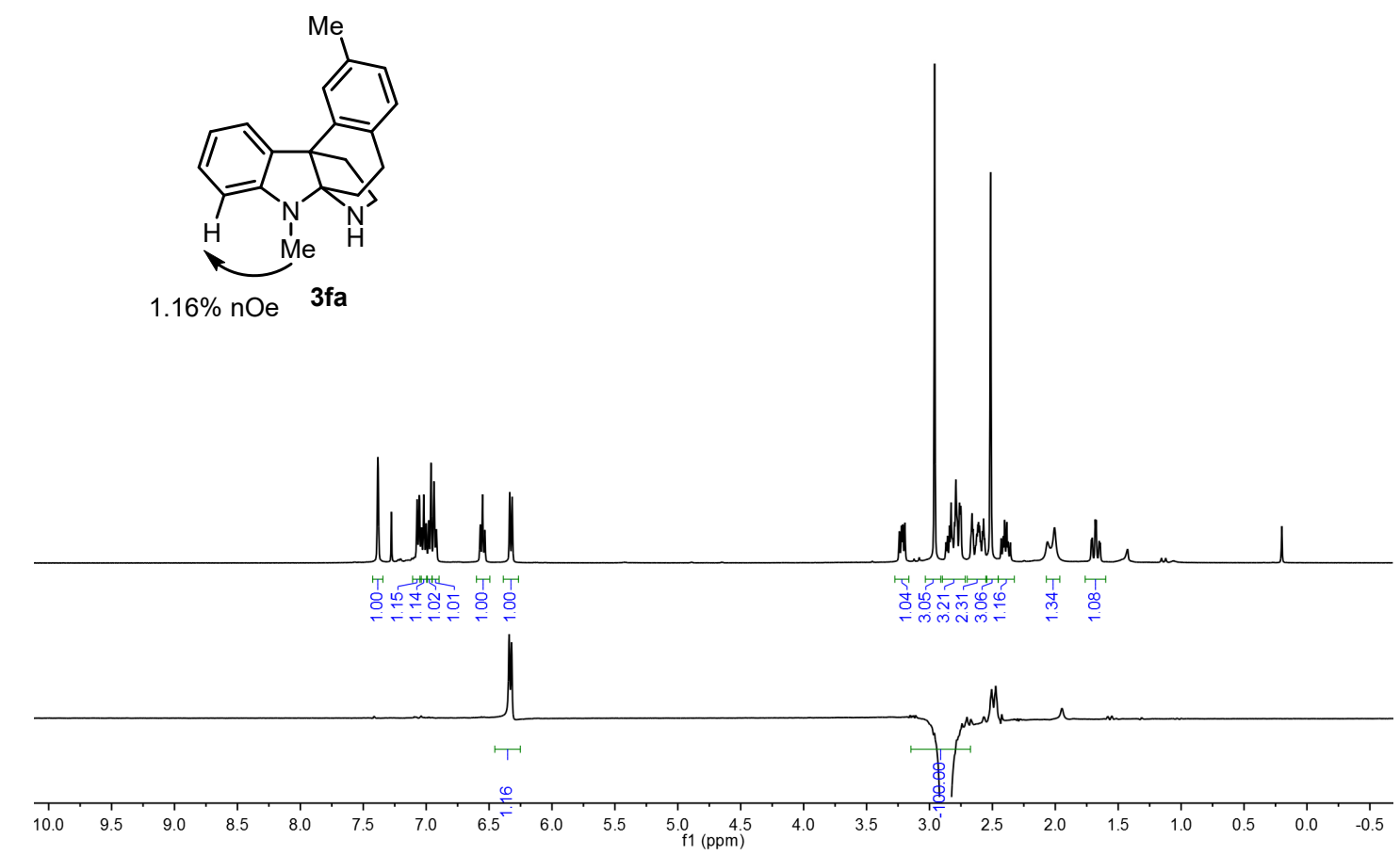

$1.16 \%$ nOe $3 f a$

${ }^{13} \mathrm{C}\left\{{ }^{1} \mathrm{H}\right\}$ NMR $\left(\mathrm{CDCl}_{3}, 100 \mathrm{MHz}\right)$ of $\mathbf{3 f a}:$

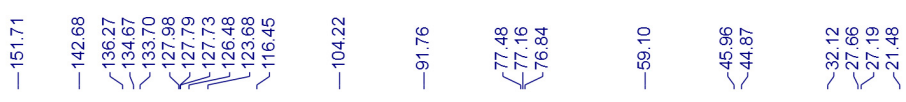

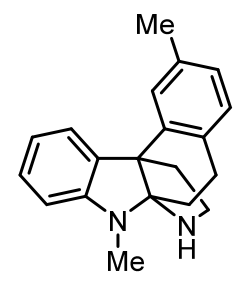

$3 f a$

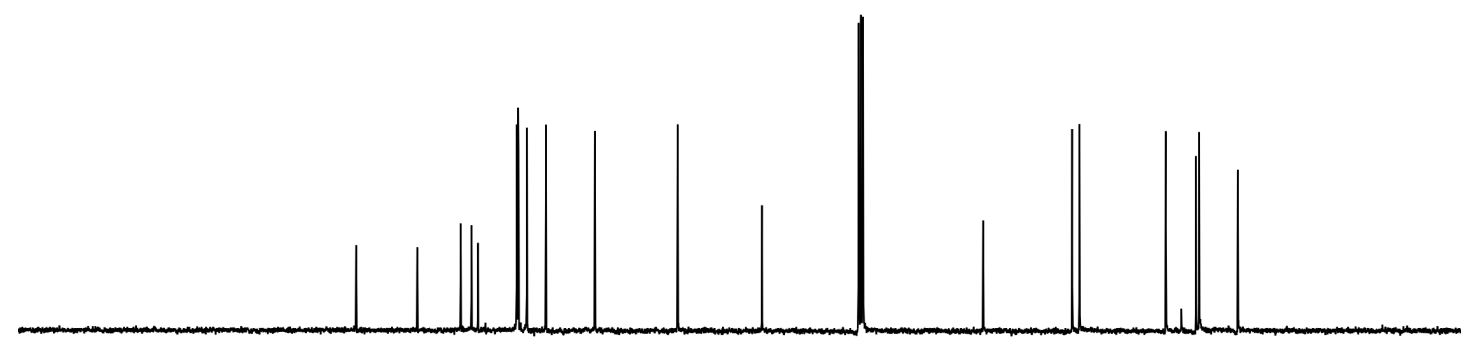

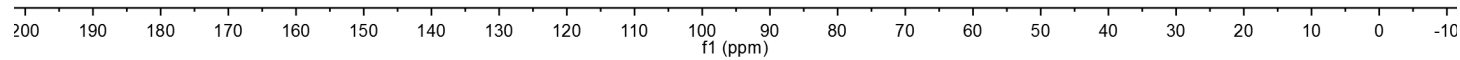


${ }^{1} \mathrm{H}$ NMR $\left(\mathrm{CDCl}_{3}, 400 \mathrm{MHz}\right)$ of $\mathbf{3 f b}$ :

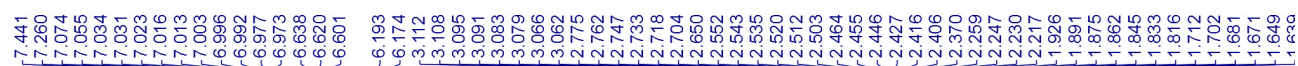

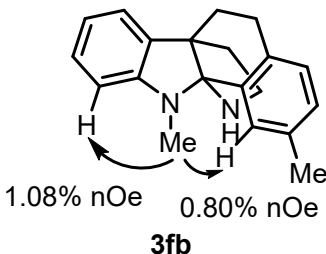

$3 \mathrm{fb}$

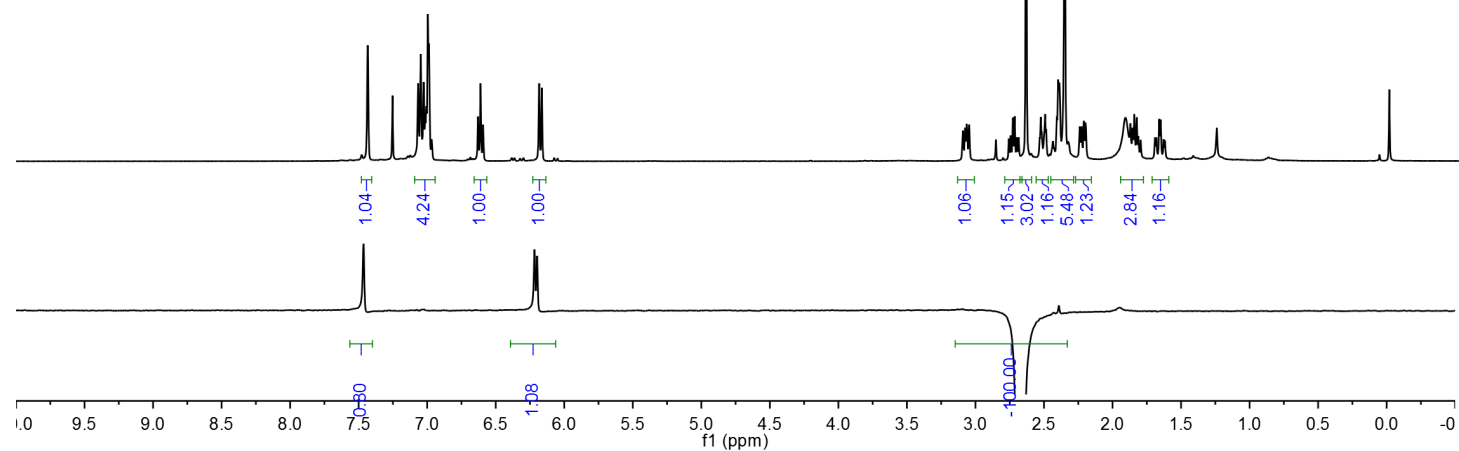

${ }^{13} \mathrm{C}\left\{{ }^{1} \mathrm{H}\right\} \mathrm{NMR}\left(\mathrm{CDCl}_{3}, 100 \mathrm{MHz}\right)$ of $\mathbf{3 f b}$ :

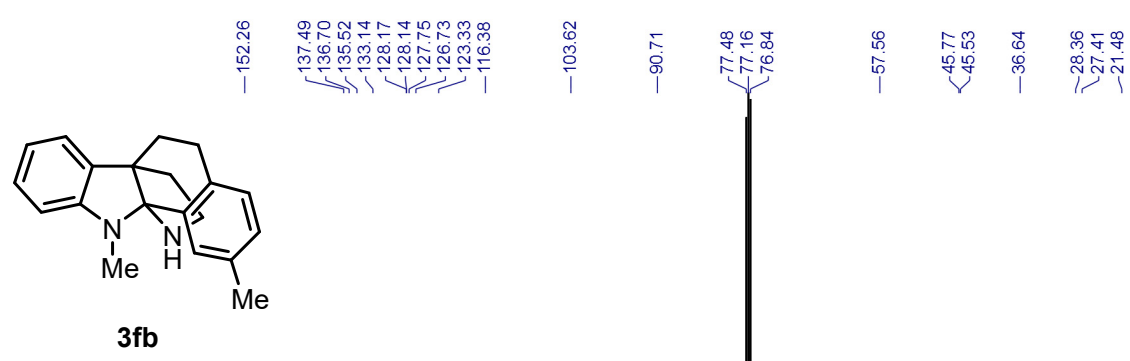

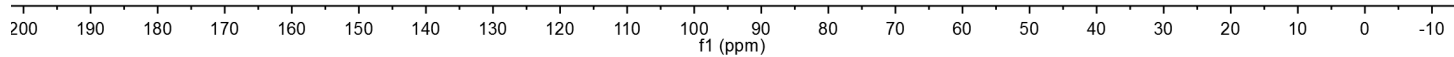


${ }^{1} \mathrm{H} \mathrm{NMR}\left(\mathrm{CDCl}_{3}, 400 \mathrm{MHz}\right)$ of $3 \mathbf{g a}:$

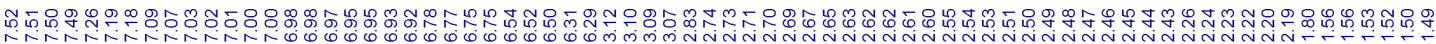
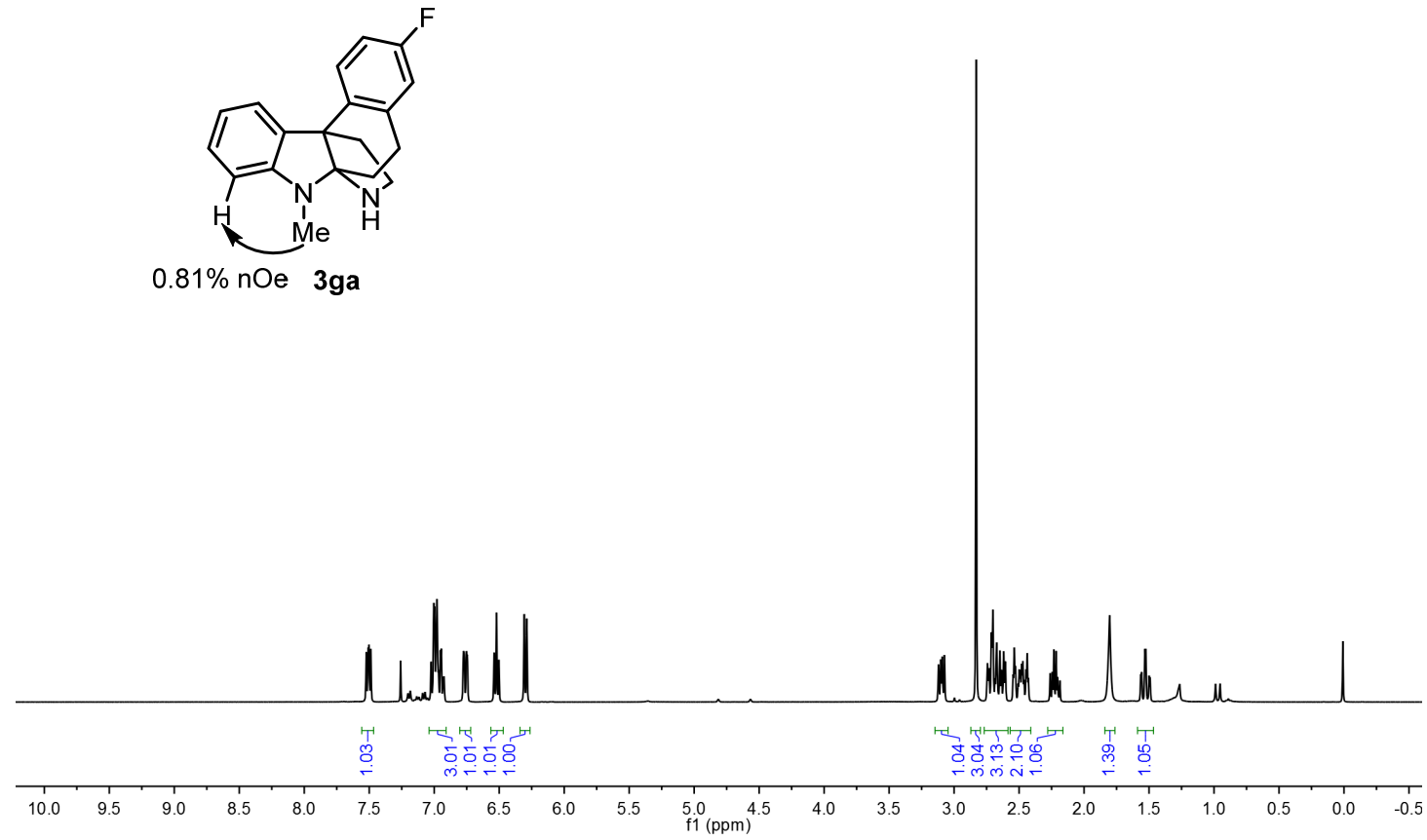

${ }^{13} \mathrm{C}\left\{{ }^{1} \mathrm{H}\right\} \mathrm{NMR}\left(\mathrm{CDCl}_{3}, 100 \mathrm{MHz}\right)$ of 3ga:
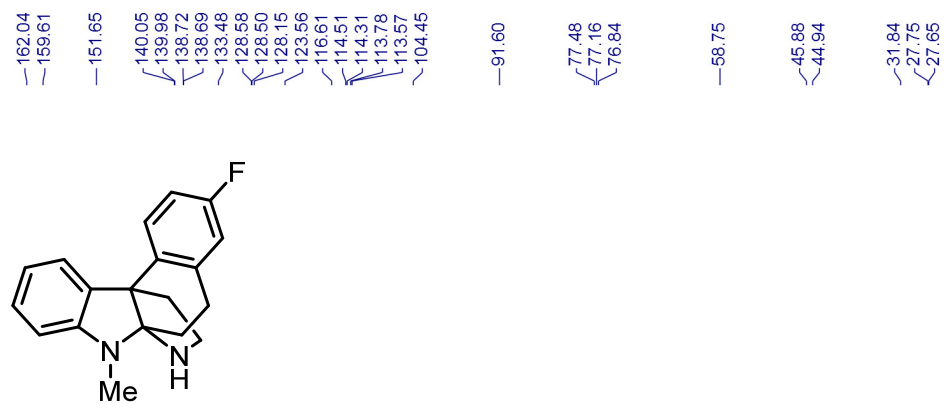

3ga

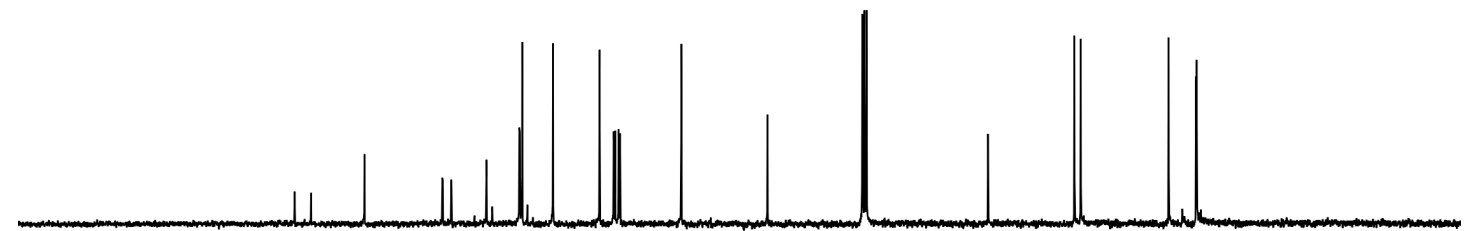

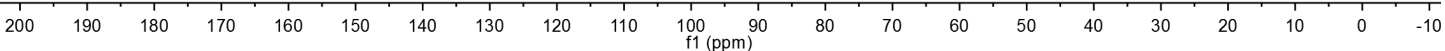


${ }^{19} \mathrm{~F}\left\{{ }^{1} \mathrm{H}\right\}$ NMR $\left(\mathrm{CDCl}_{3}, 386 \mathrm{MHz}\right)$ of 3ga:

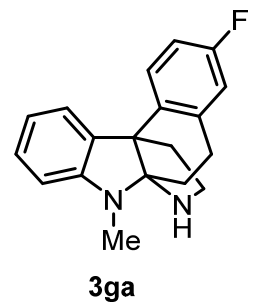

$\begin{array}{llllllllllllllllllllllllllll}10 & 0 & -10 & -20 & -30 & -40 & -50 & -60 & -70 & -80 & -90 & -100 & 1 & 110 & 120 & 130 & -140 & -150 & -160 & -170 & -180 & -190 & -200 & -210\end{array}$ 
${ }^{1} \mathrm{H} \mathrm{NMR}\left(\mathrm{CDCl}_{3}, 400 \mathrm{MHz}\right)$ of $\mathbf{3 g b}$ :

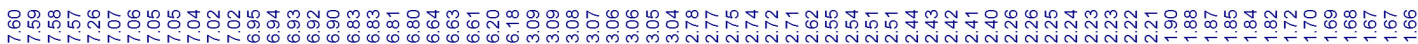

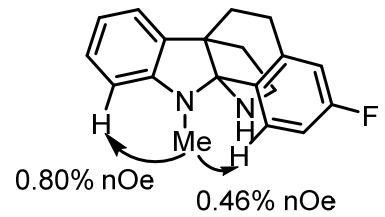

$3 \mathrm{gb}$

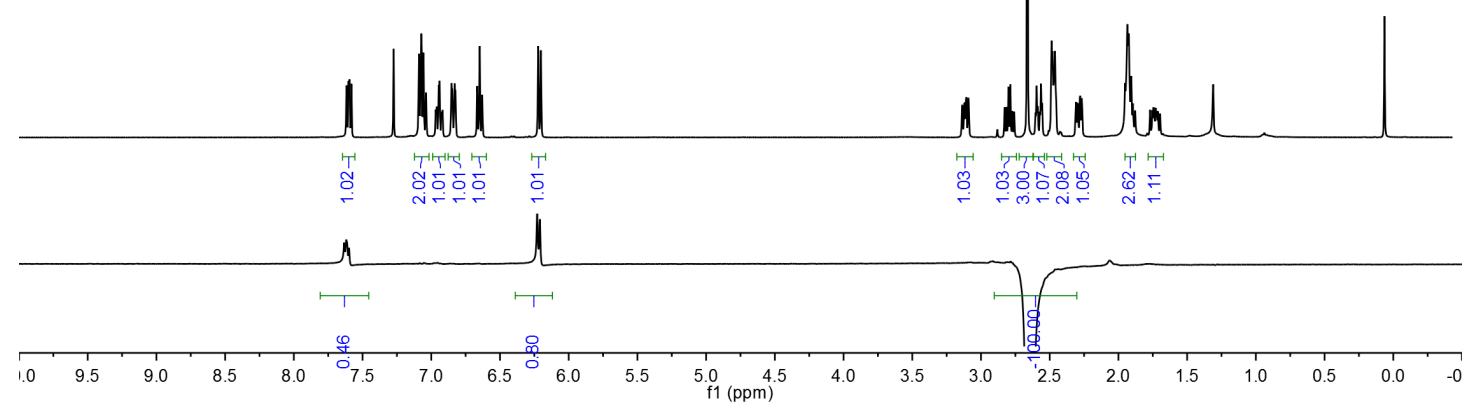

${ }^{13} \mathrm{C}\left\{{ }^{1} \mathrm{H}\right\} \mathrm{NMR}\left(\mathrm{CDCl}_{3}, 100 \mathrm{MHz}\right)$ of $\mathbf{3 g b}$ :

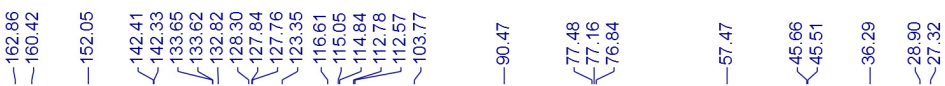
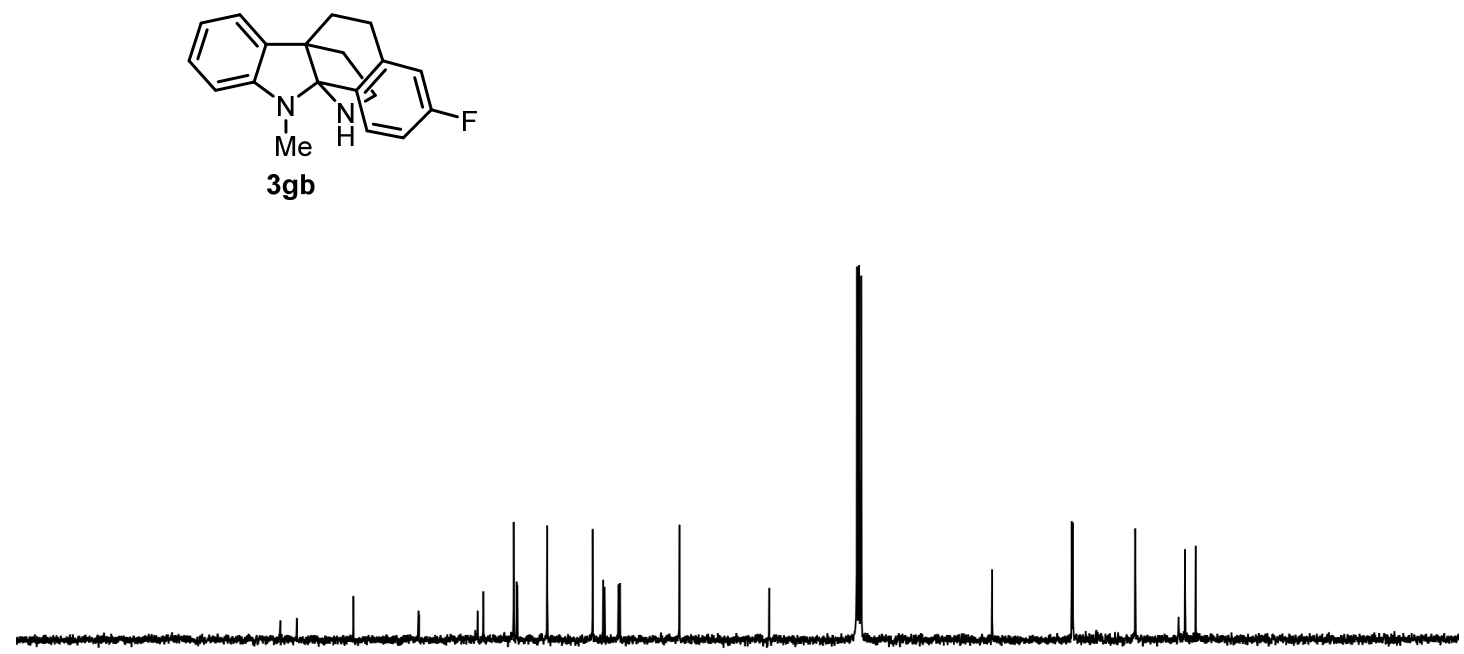

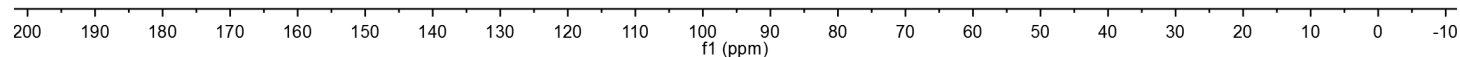


${ }^{19} \mathrm{~F}\left\{{ }^{1} \mathrm{H}\right\}$ NMR $\left(\mathrm{CDCl}_{3}, 386 \mathrm{MHz}\right)$ of $\mathbf{3 g b}$ :

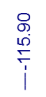

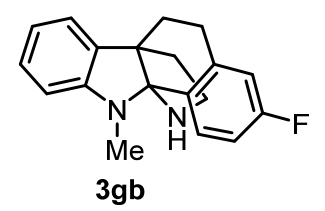

$\begin{array}{llllllllllllllllllllllllllll}10 & 0 & -10 & -20 & -30 & -40 & -50 & -60 & -70 & -80 & -90 & -100 & -110 & -120 & -130 & -140 & -150 & -160 & -170 & -180 & -190 & -200 & -210\end{array}$ 
${ }^{1} \mathrm{H} \mathrm{NMR}\left(\mathrm{CDCl}_{3}, 400 \mathrm{MHz}\right)$ of 3ha:

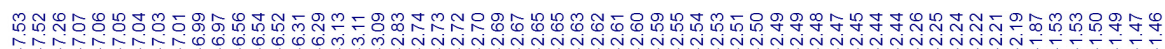

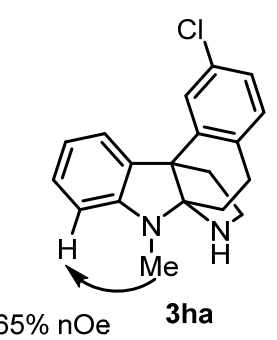

$1.65 \% \mathrm{nOe}$

3ha
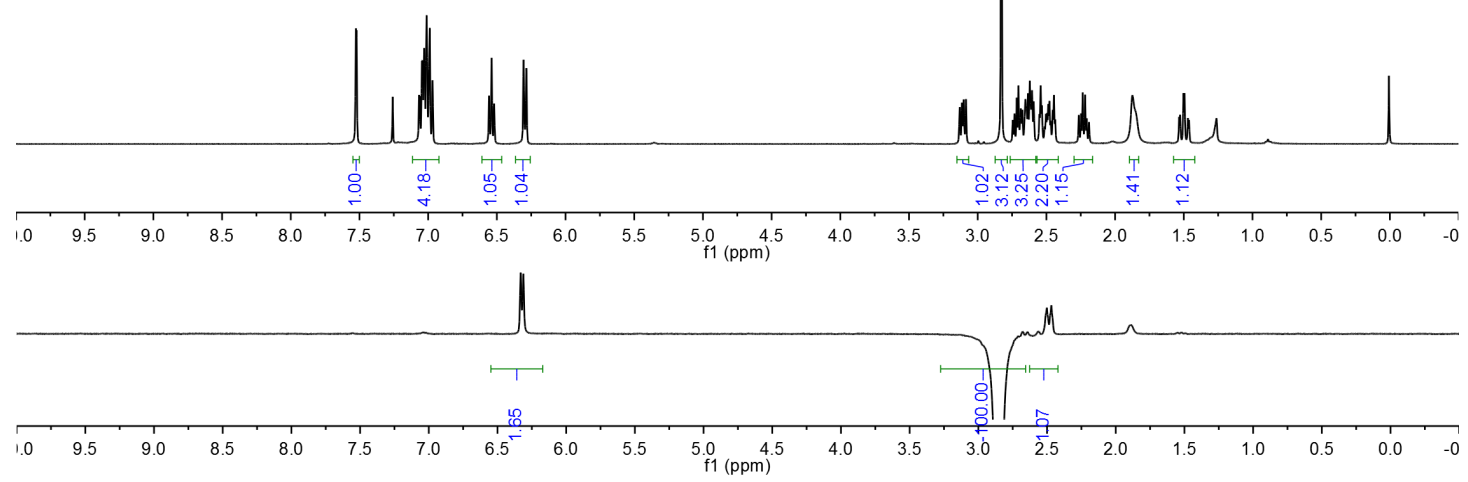

${ }^{13} \mathrm{C}\left\{{ }^{1} \mathrm{H}\right\} \mathrm{NMR}\left(\mathrm{CDCl}_{3}, 100 \mathrm{MHz}\right)$ of $\mathbf{3 h a}:$
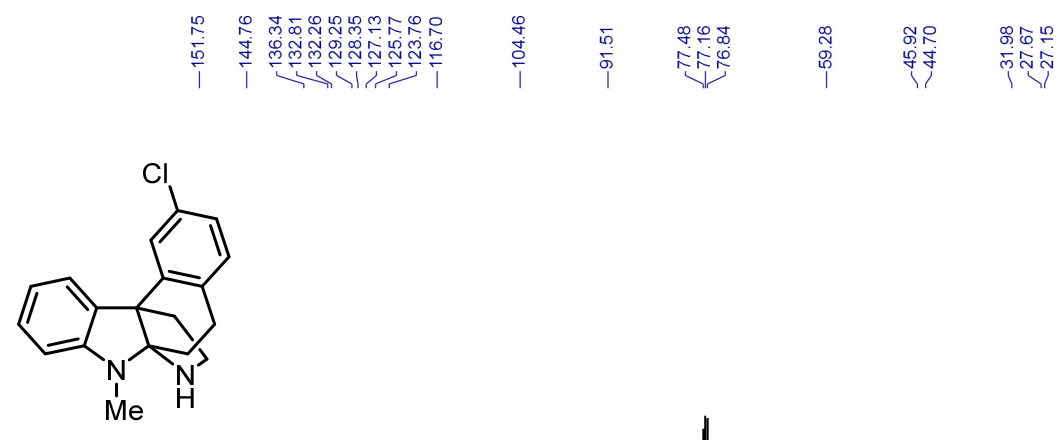

3ha

\begin{tabular}{rllllllllll}
\hline 200 & 190 & 180 & 170 & 160 & 150 & 140 & 130 & 120 & 110 & $\underset{f}{100}(\mathrm{ppm})$
\end{tabular} 
${ }^{1} \mathrm{H} \mathrm{NMR}\left(\mathrm{CDCl}_{3}, 400 \mathrm{MHz}\right)$ of $\mathbf{3 h b}$ :

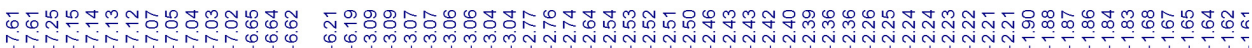

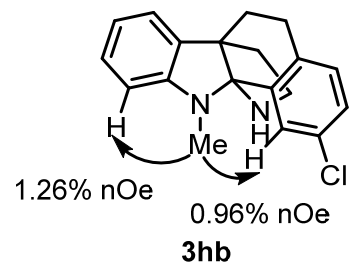

$3 \mathrm{hb}$

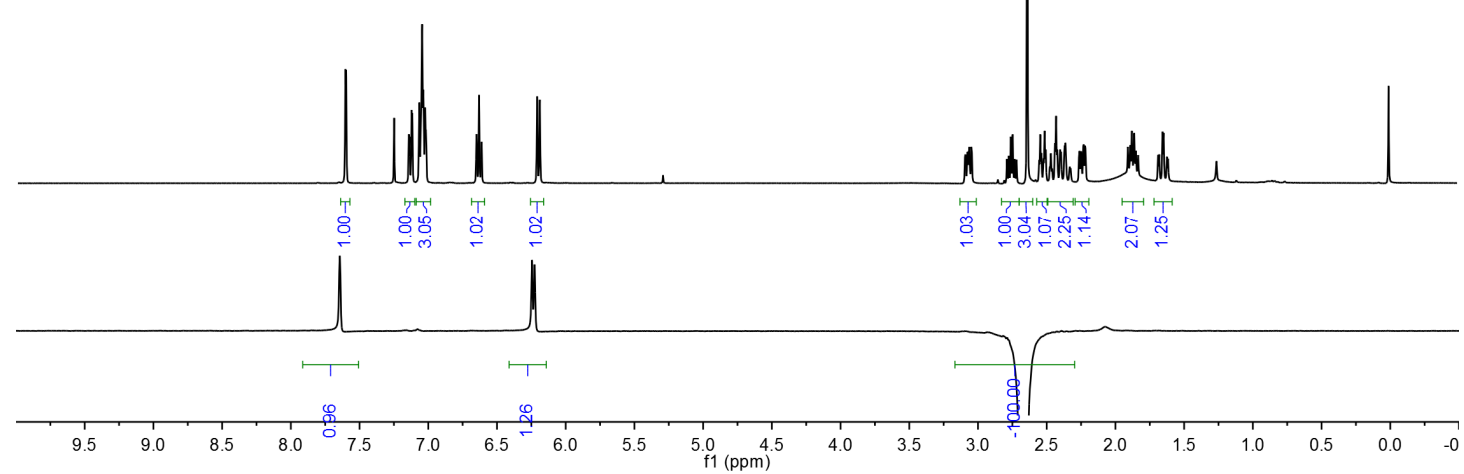

${ }^{13} \mathrm{C}\left\{{ }^{1} \mathrm{H}\right\} \mathrm{NMR}\left(\mathrm{CDCl}_{3}, 100 \mathrm{MHz}\right)$ of $\mathbf{3 h b}$ :

|

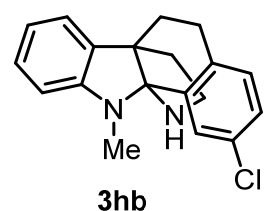

3h
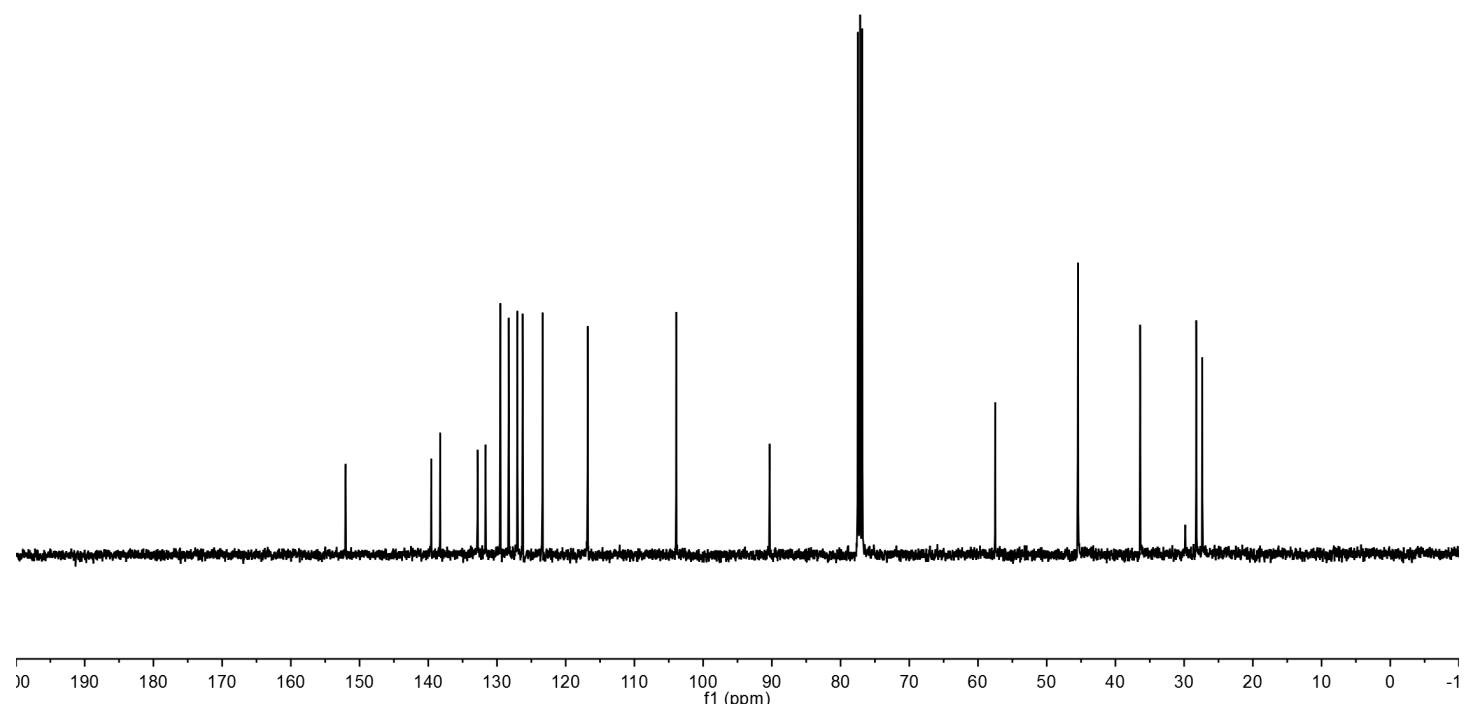
${ }^{1} \mathrm{H}$ NMR $\left(\mathrm{CDCl}_{3}, 400 \mathrm{MHz}\right)$ of 3ia:

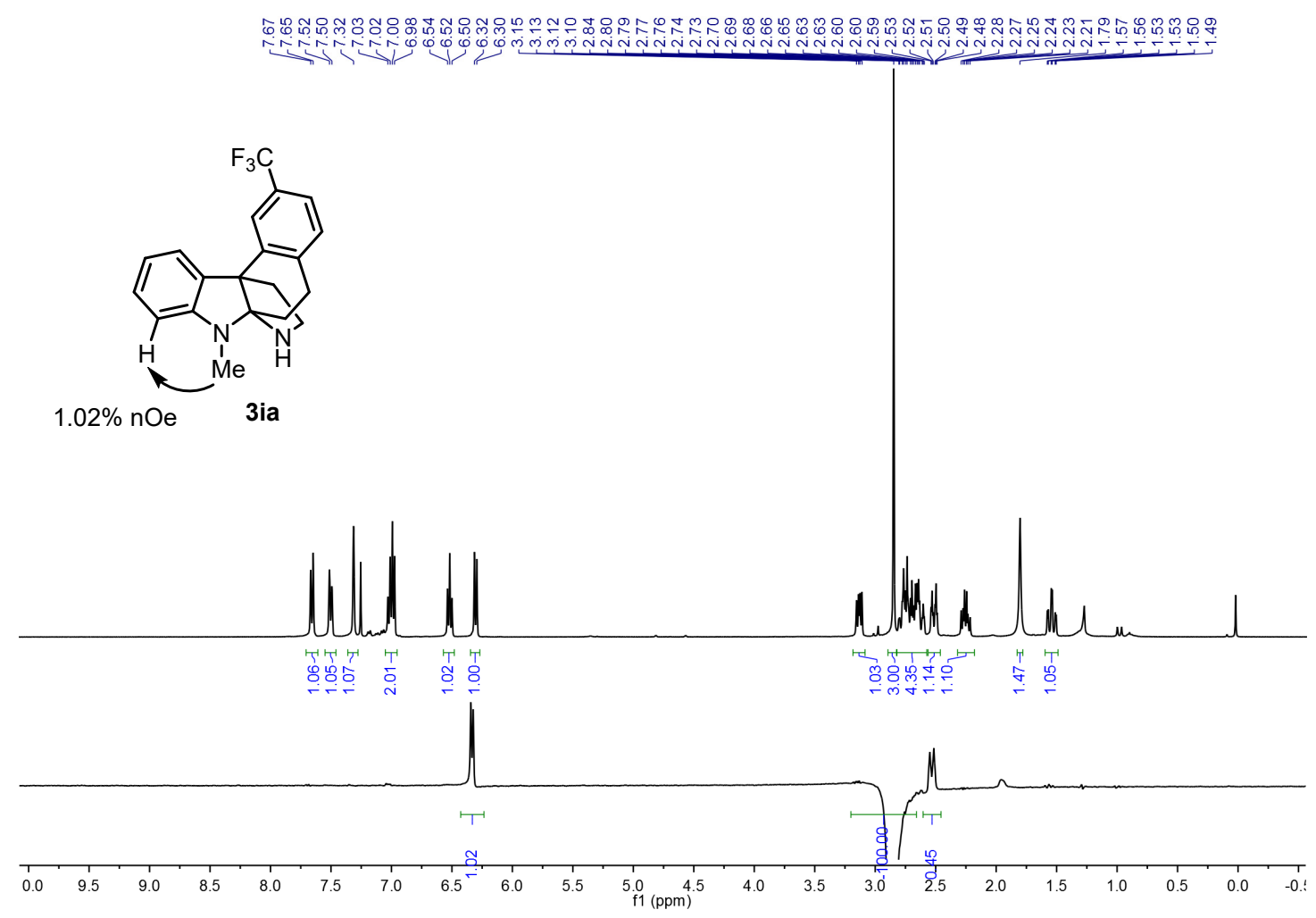

${ }^{13} \mathrm{C}\left\{{ }^{1} \mathrm{H}\right\}$ NMR $\left(\mathrm{CDCl}_{3}, 100 \mathrm{MHz}\right)$ of 3ia:
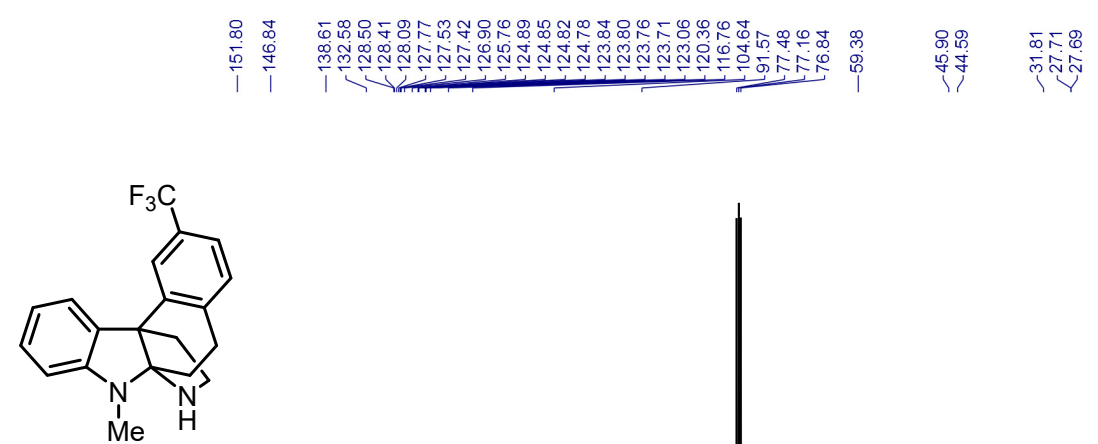

3ia

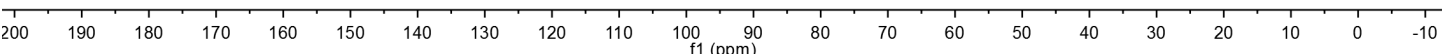


${ }^{19} \mathrm{~F}\left\{{ }^{1} \mathrm{H}\right\}$ NMR $\left(\mathrm{CDCl}_{3}, 386 \mathrm{MHz}\right)$ of 3ia:

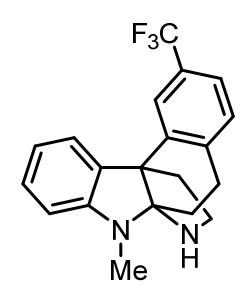

3ia

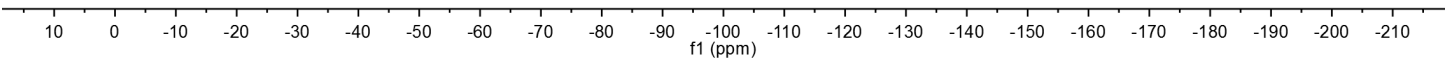


${ }^{1} \mathrm{H}$ NMR $\left(\mathrm{CDCl}_{3}, 400 \mathrm{MHz}\right)$ of 3ib:

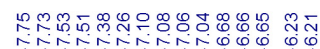

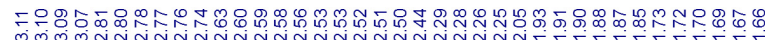

rivinining

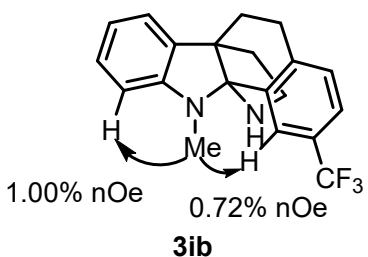

$3 \mathrm{ib}$
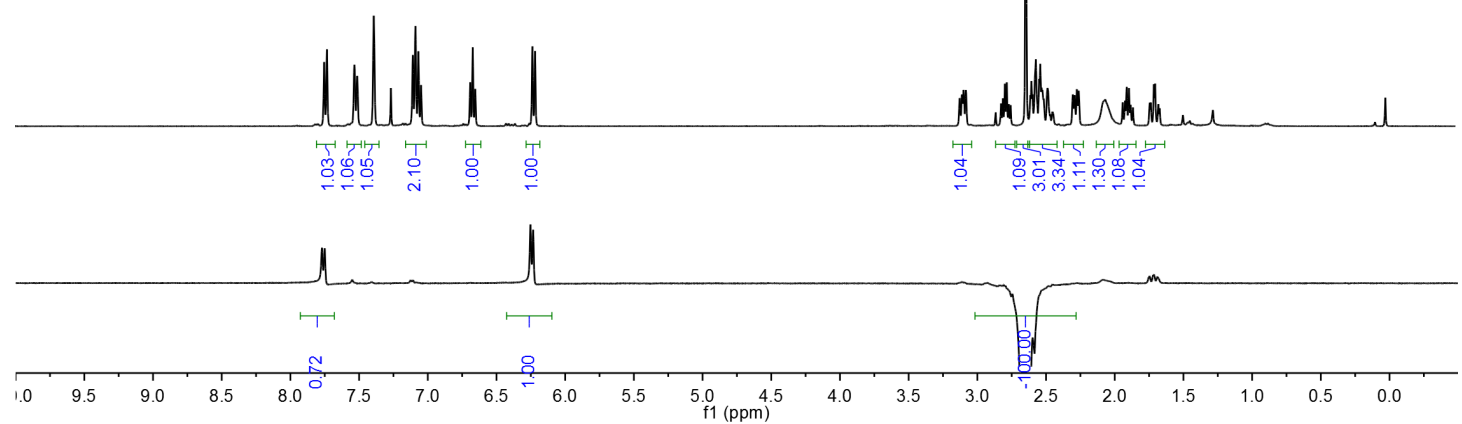

${ }^{13} \mathrm{C}\left\{{ }^{1} \mathrm{H}\right\} \mathrm{NMR}\left(\mathrm{CDCl}_{3}, 100 \mathrm{MHz}\right)$ of $\mathbf{3 i b}$ :

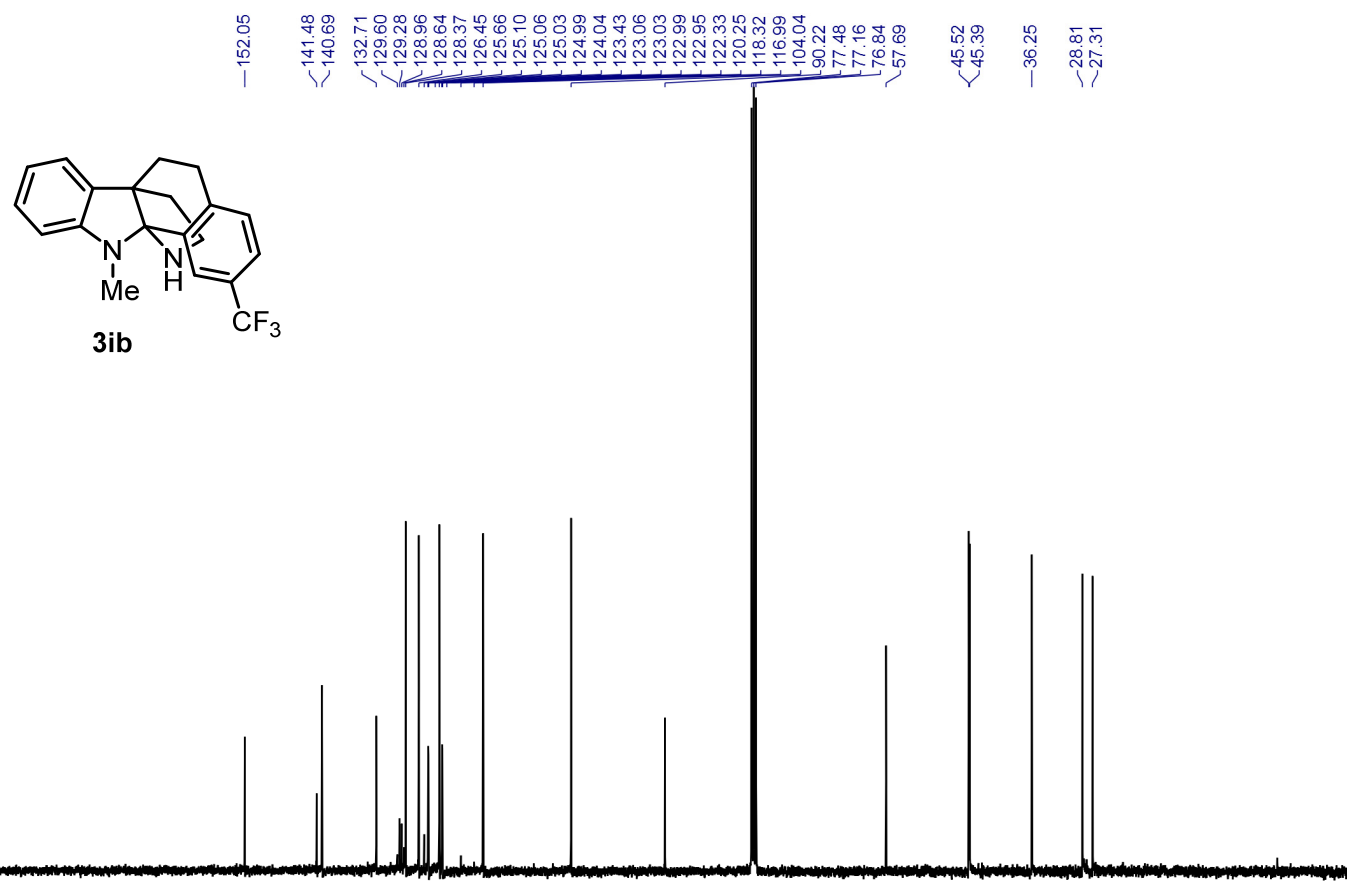

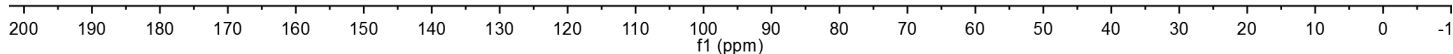


${ }^{19} \mathrm{~F}\left\{{ }^{1} \mathrm{H}\right\}$ NMR $\left(\mathrm{CDCl}_{3}, 386 \mathrm{MHz}\right)$ of 3ib:

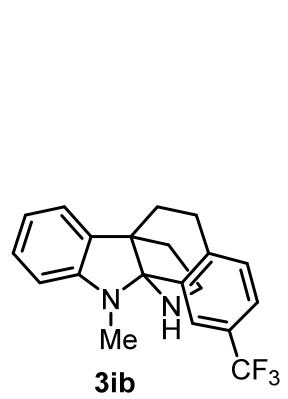

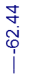

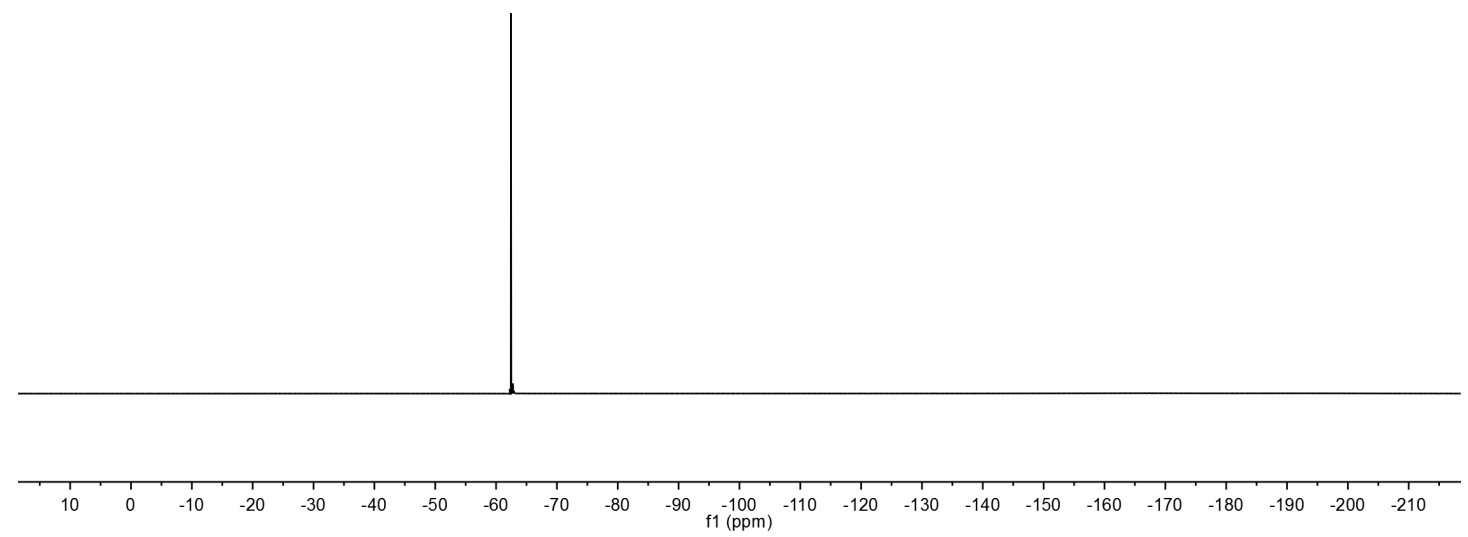


${ }^{1} \mathrm{H} \mathrm{NMR}\left(\mathrm{CDCl}_{3}, 400 \mathrm{MHz}\right)$ of $\mathbf{3 j b}$ :

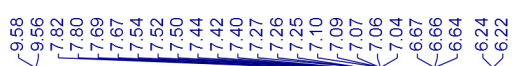

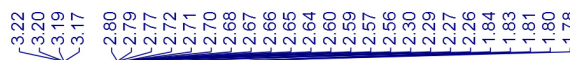
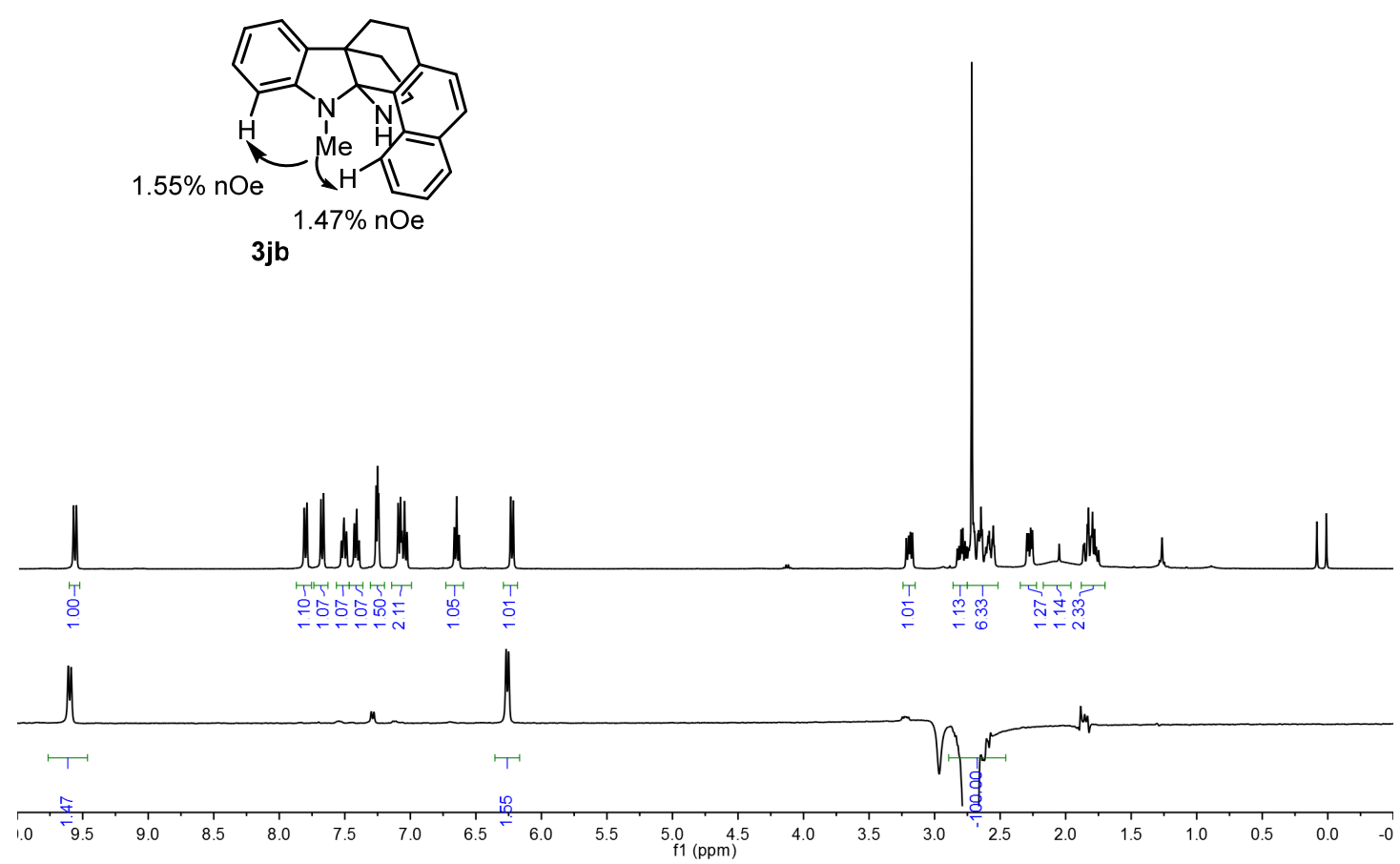

${ }^{13} \mathrm{C}\left\{{ }^{1} \mathrm{H}\right\} \mathrm{NMR}\left(\mathrm{CDCl}_{3}, 100 \mathrm{MHz}\right)$ of $\mathbf{3 j b}$ :

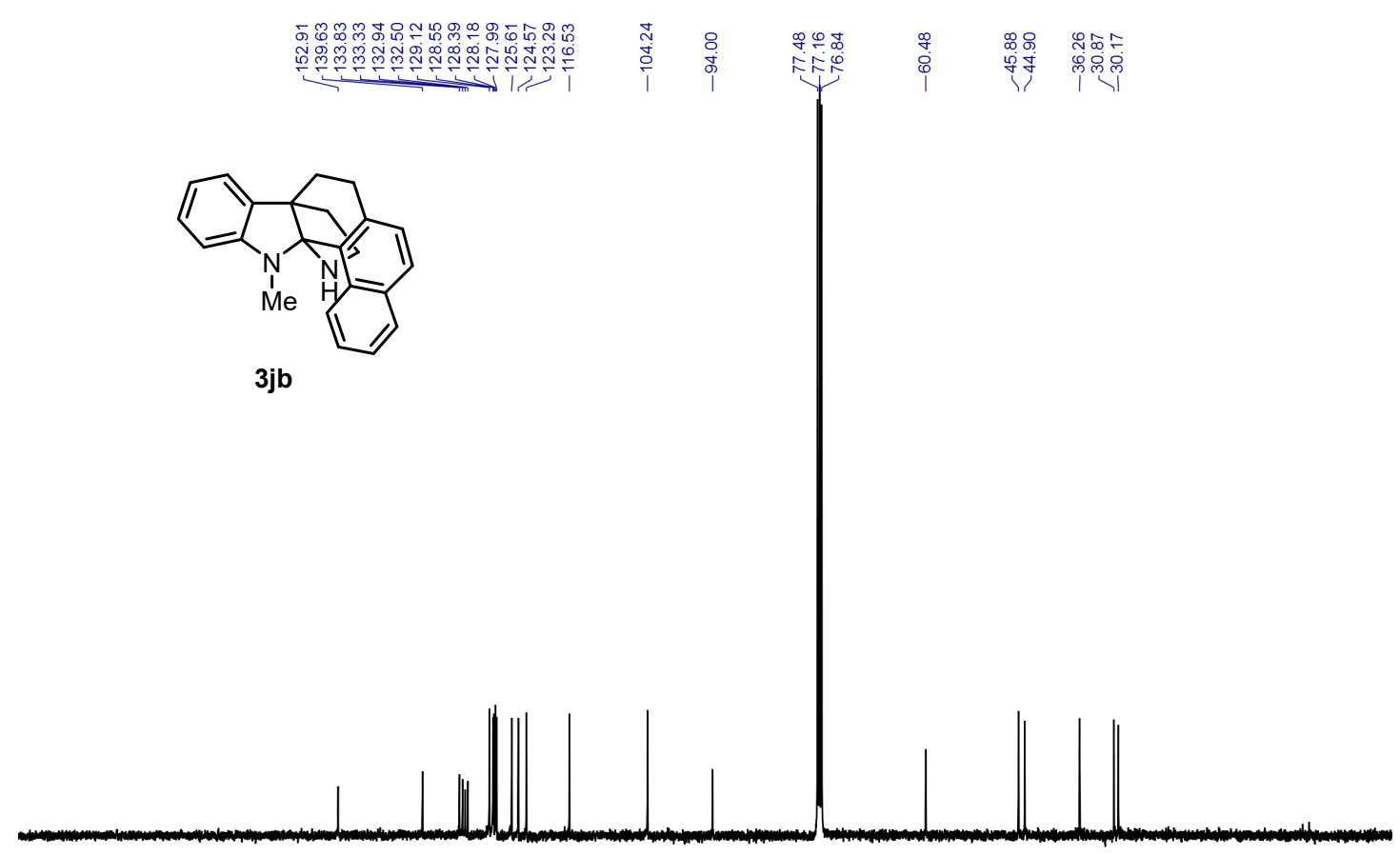

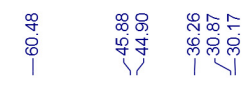

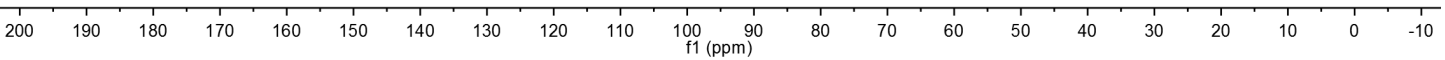


${ }^{1} \mathrm{H} \mathrm{NMR}\left(\mathrm{CDCl}_{3}, 400 \mathrm{MHz}\right)$ of 3la:

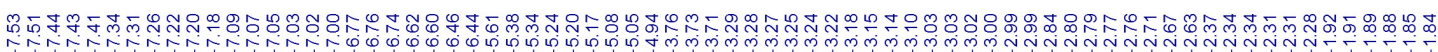

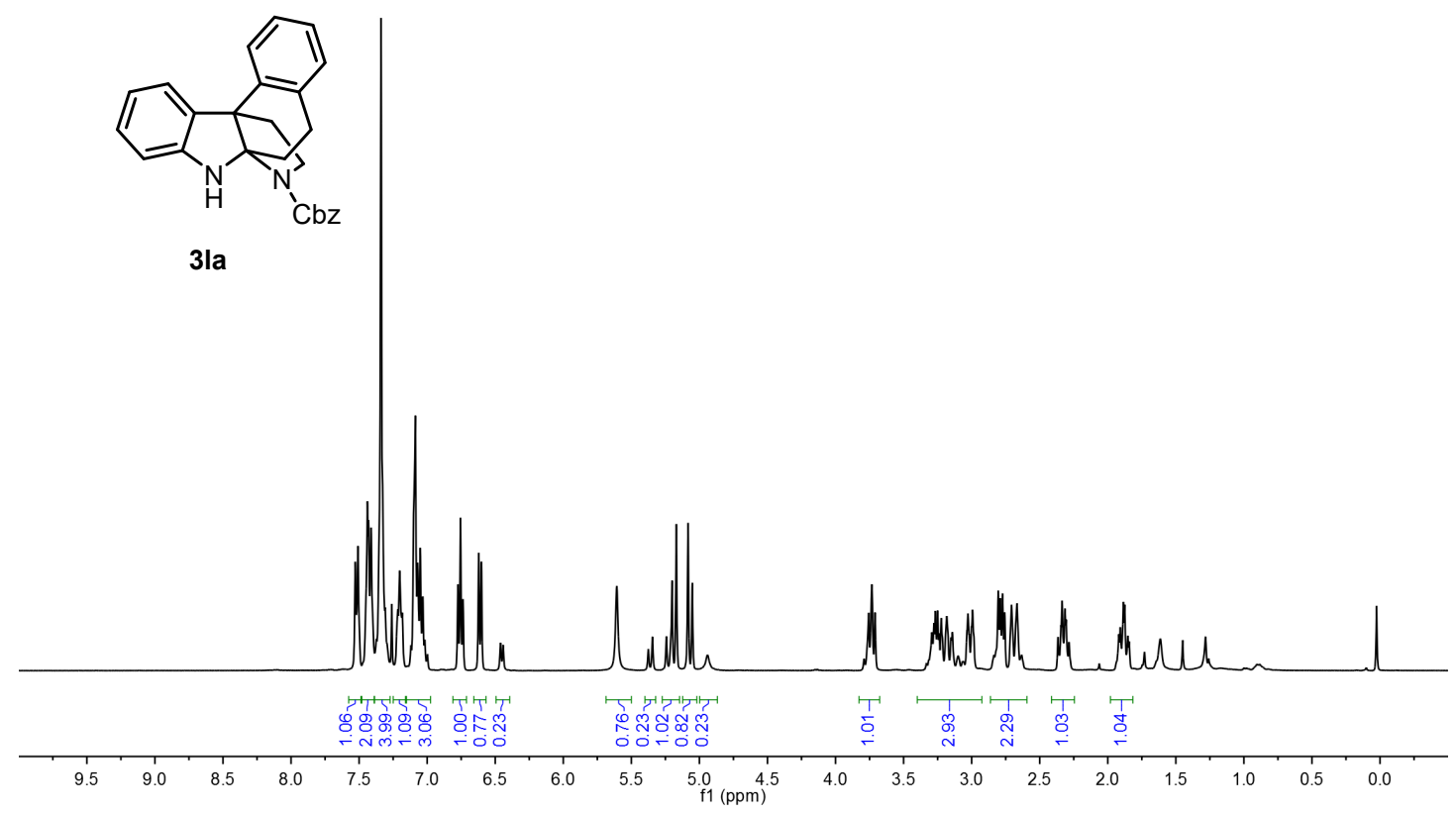

${ }^{13} \mathrm{C}\left\{{ }^{1} \mathrm{H}\right\} \mathrm{NMR}\left(\mathrm{CDCl}_{3}, 100 \mathrm{MHz}\right)$ of 3la:

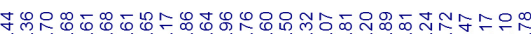

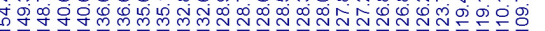

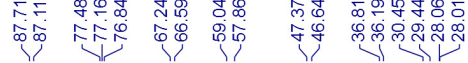

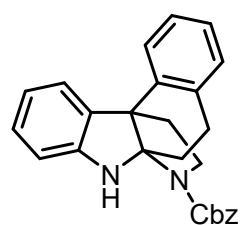

3la
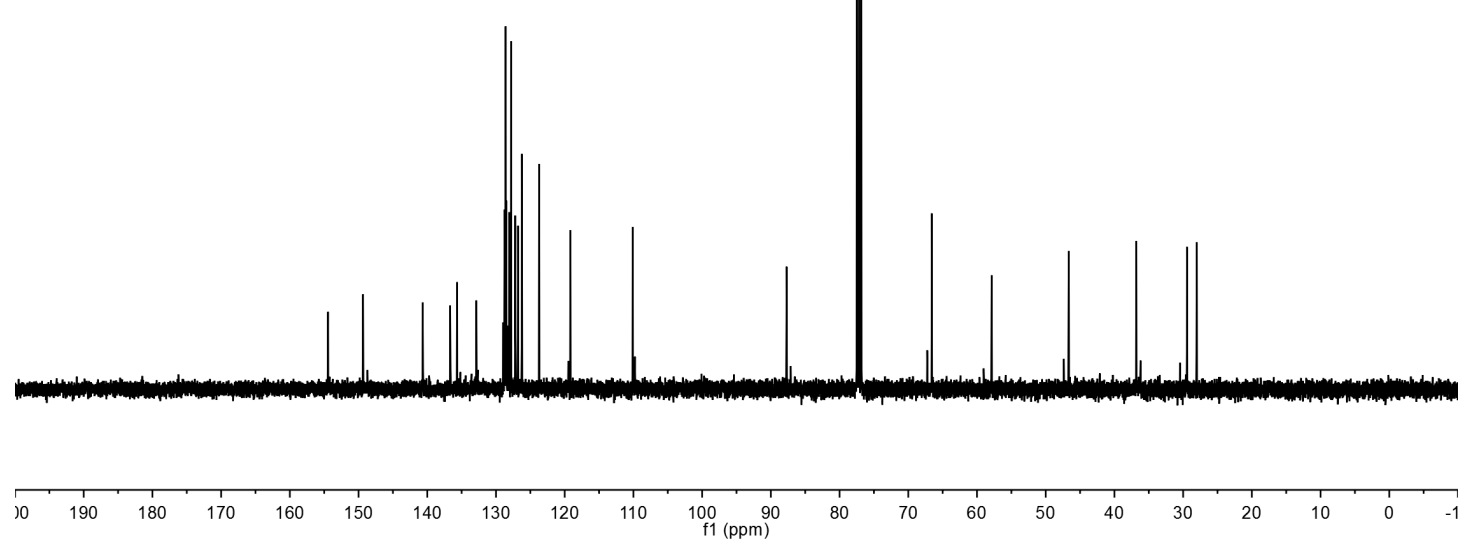
2D HSQC of 3la:

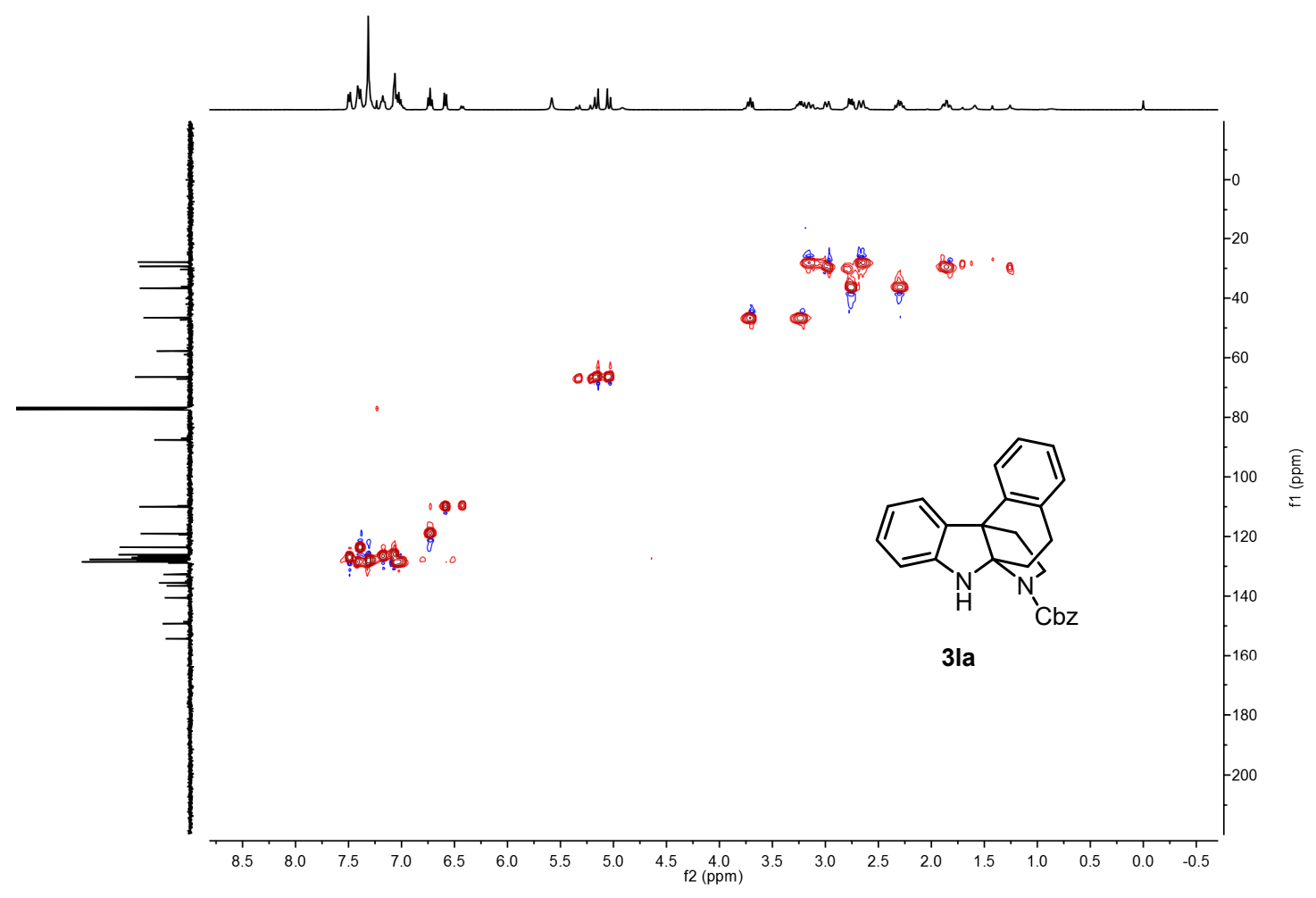

2D HMBC of 3la:

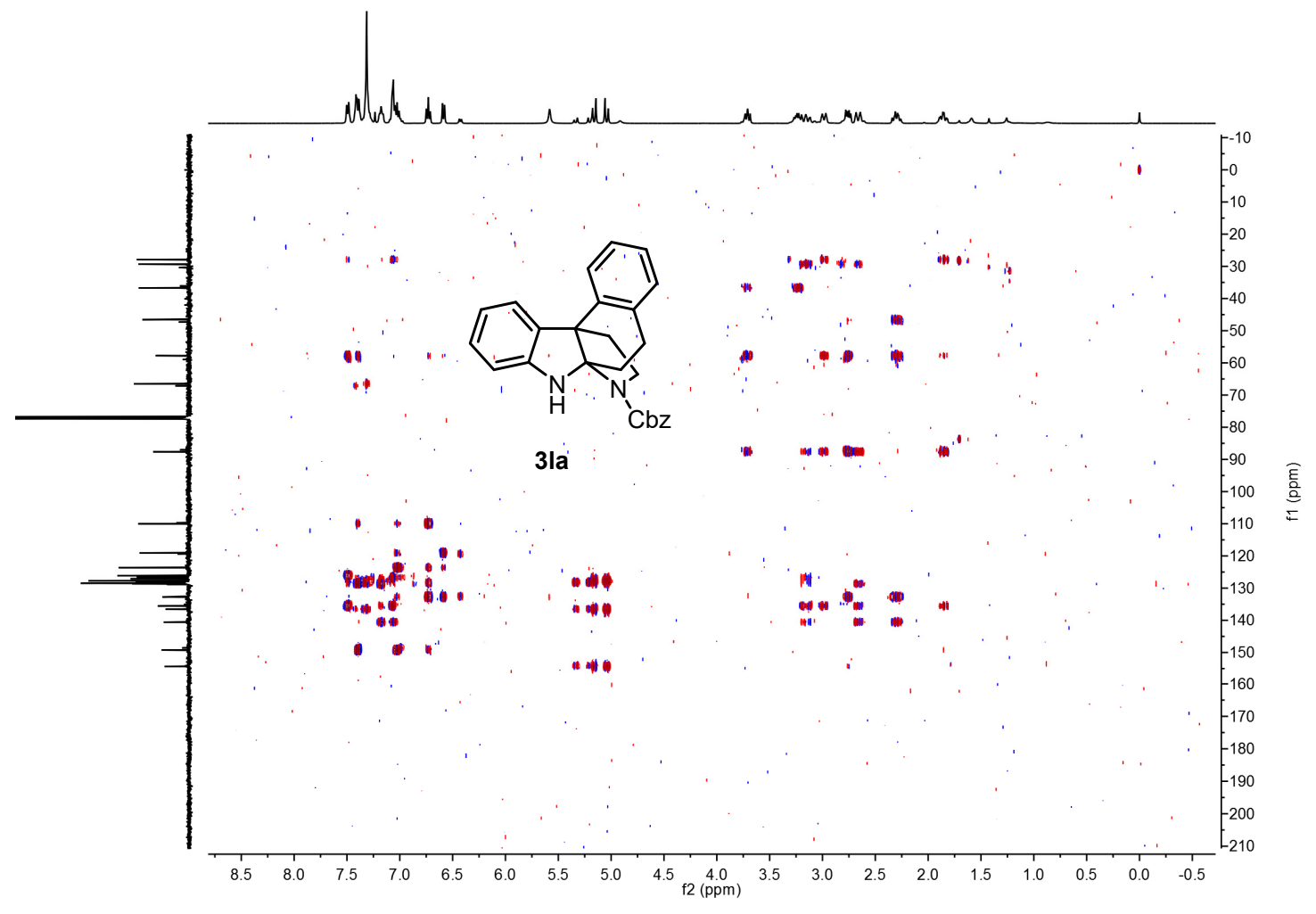


${ }^{1} \mathrm{H} \mathrm{NMR}\left(\mathrm{CDCl}_{3}, 400 \mathrm{MHz}\right)$ of $\mathbf{3 m a}$ :

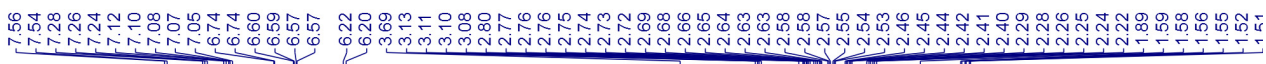

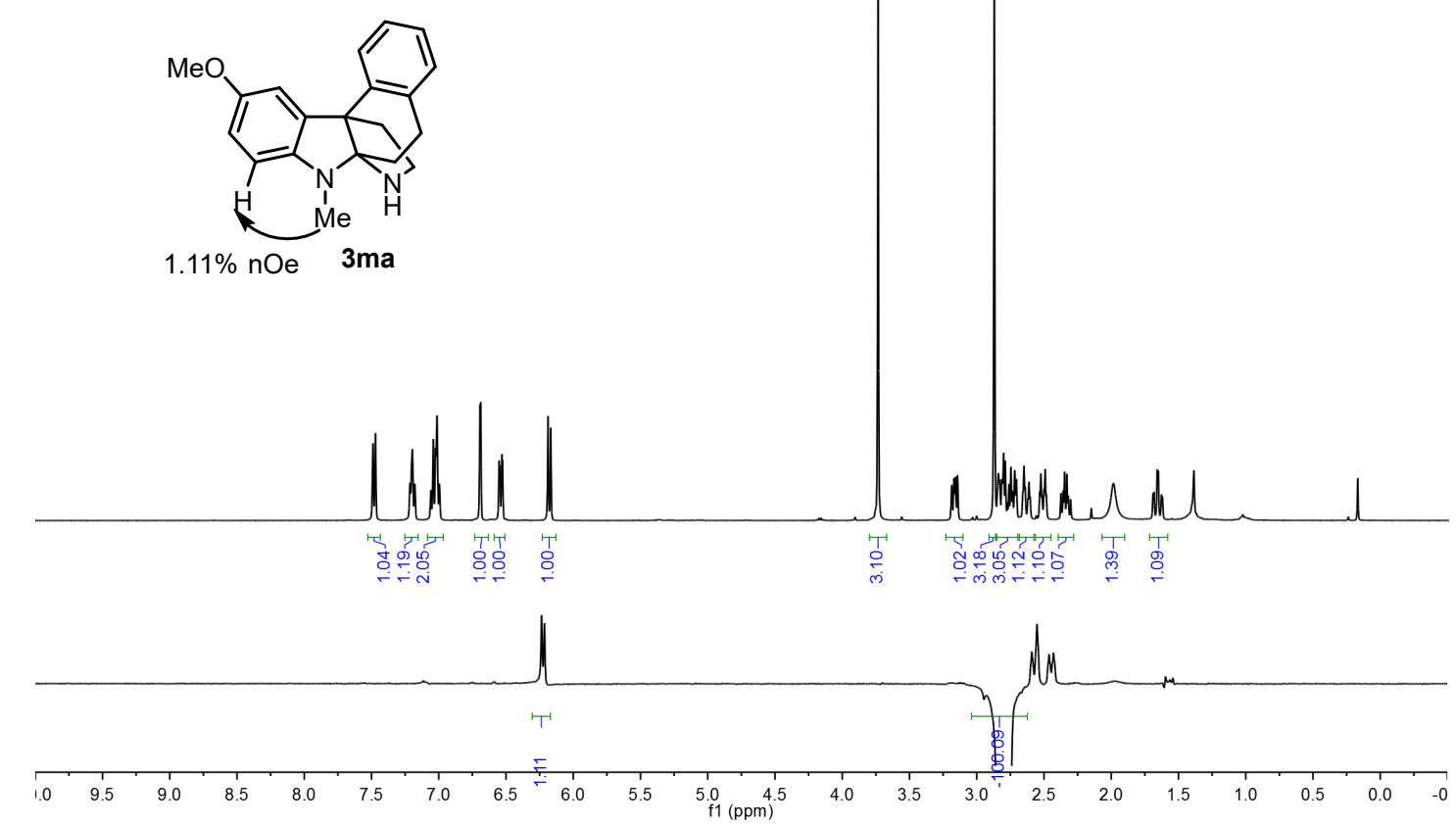

${ }^{13} \mathrm{C}\left\{{ }^{1} \mathrm{H}\right\}$ NMR $\left(\mathrm{CDCl}_{3}, 100 \mathrm{MHz}\right)$ of $\mathbf{3 m a}:$

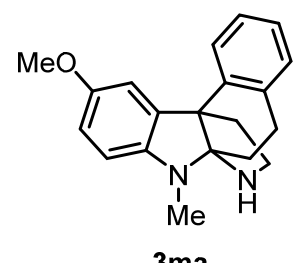

$3 \mathrm{ma}$
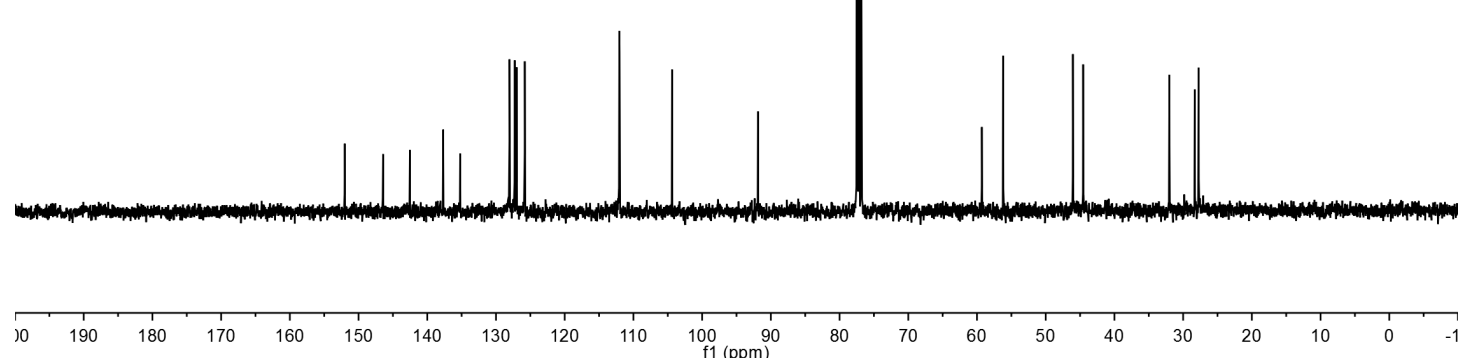
${ }^{1} \mathrm{H} \mathrm{NMR}\left(\mathrm{CDCl}_{3}, 400 \mathrm{MHz}\right)$ of $\mathbf{3 m b}$ :

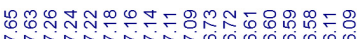

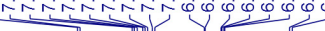

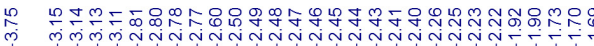
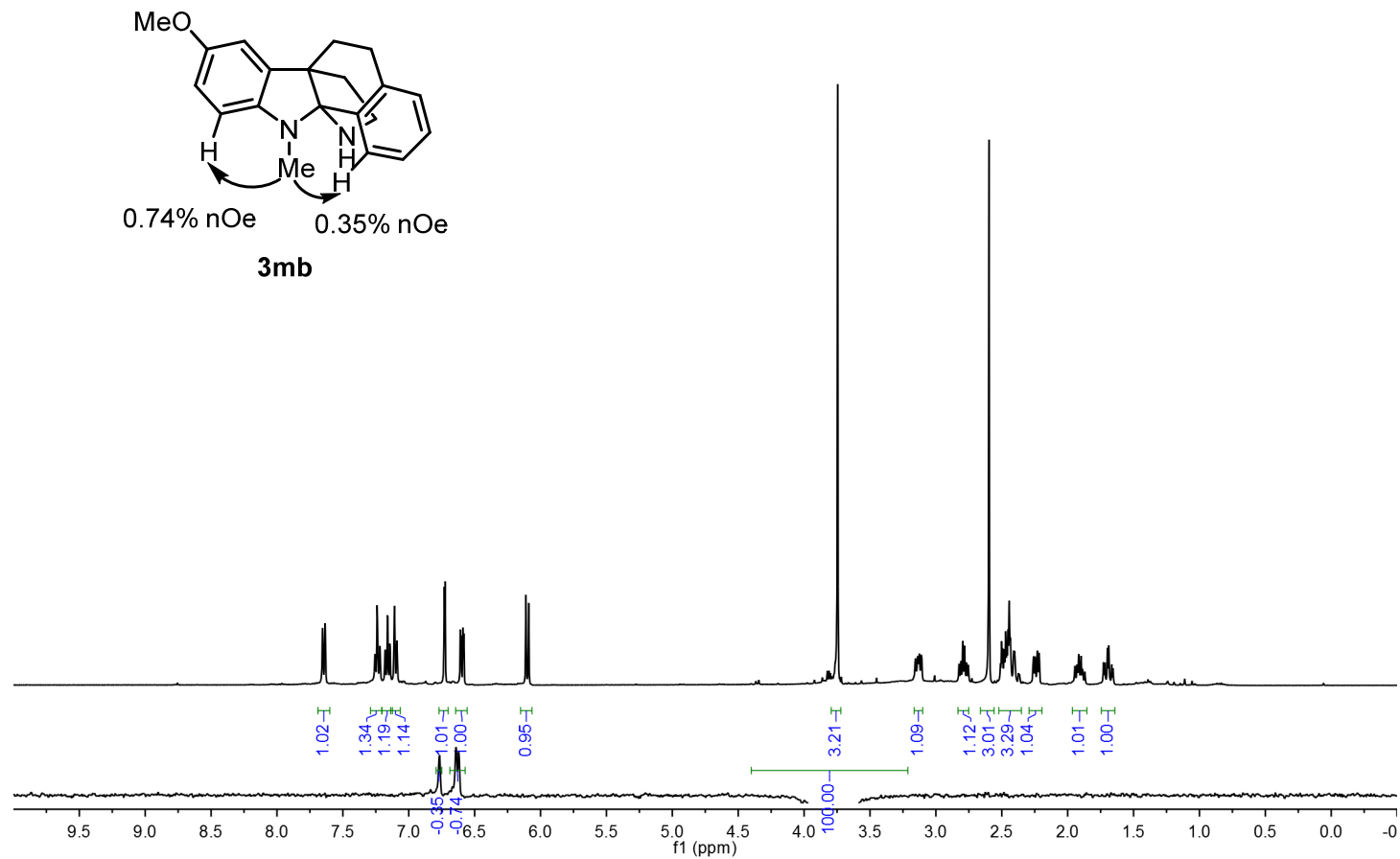

${ }^{13} \mathrm{C}\left\{{ }^{1} \mathrm{H}\right\}$ NMR $\left(\mathrm{CDCl}_{3}, 100 \mathrm{MHz}\right)$ of $\mathbf{3 m b}$ :

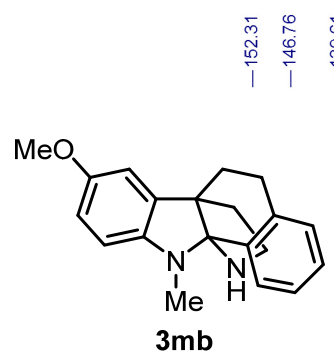

i l
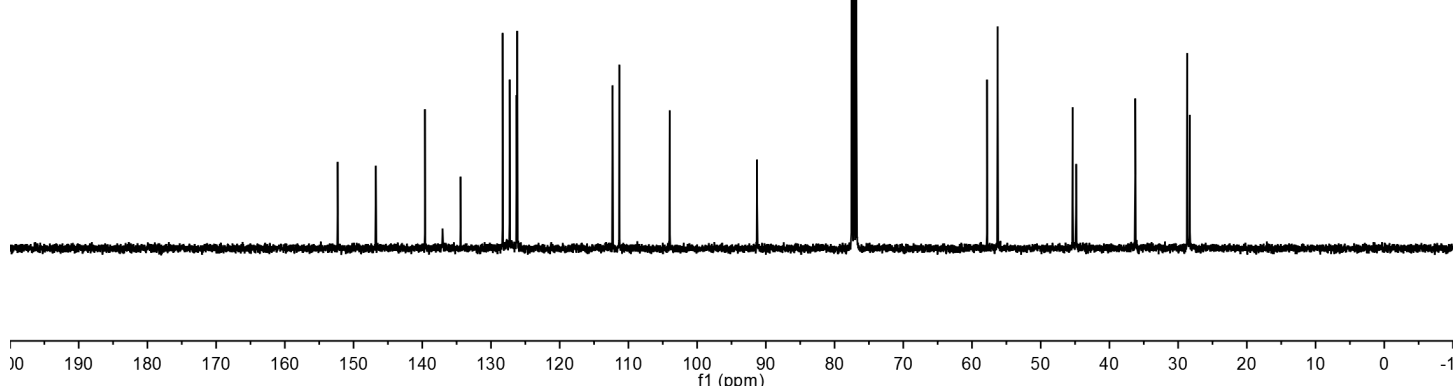
${ }^{1} \mathrm{H}$ NMR $\left(\mathrm{CDCl}_{3}, 400 \mathrm{MHz}\right)$ of $\mathbf{2 n}$ :

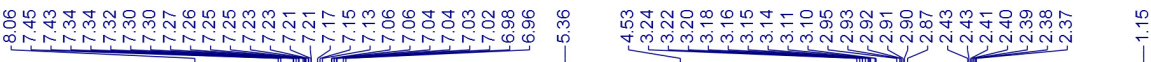

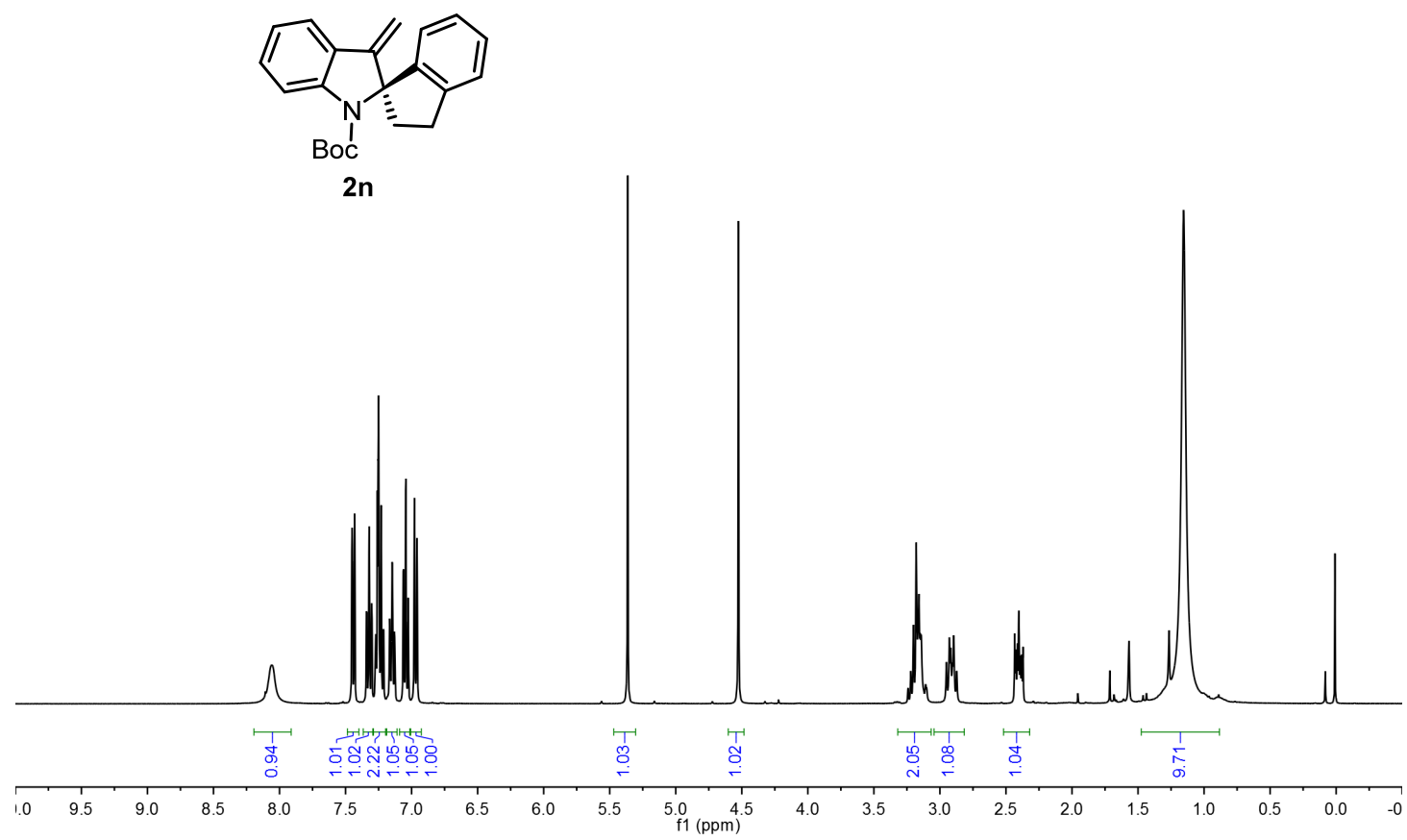

${ }^{13} \mathrm{C}\left\{{ }^{1} \mathrm{H}\right\}$ NMR $\left(\mathrm{CDCl}_{3}, 100 \mathrm{MHz}\right)$ of $\mathbf{2 n}$ :
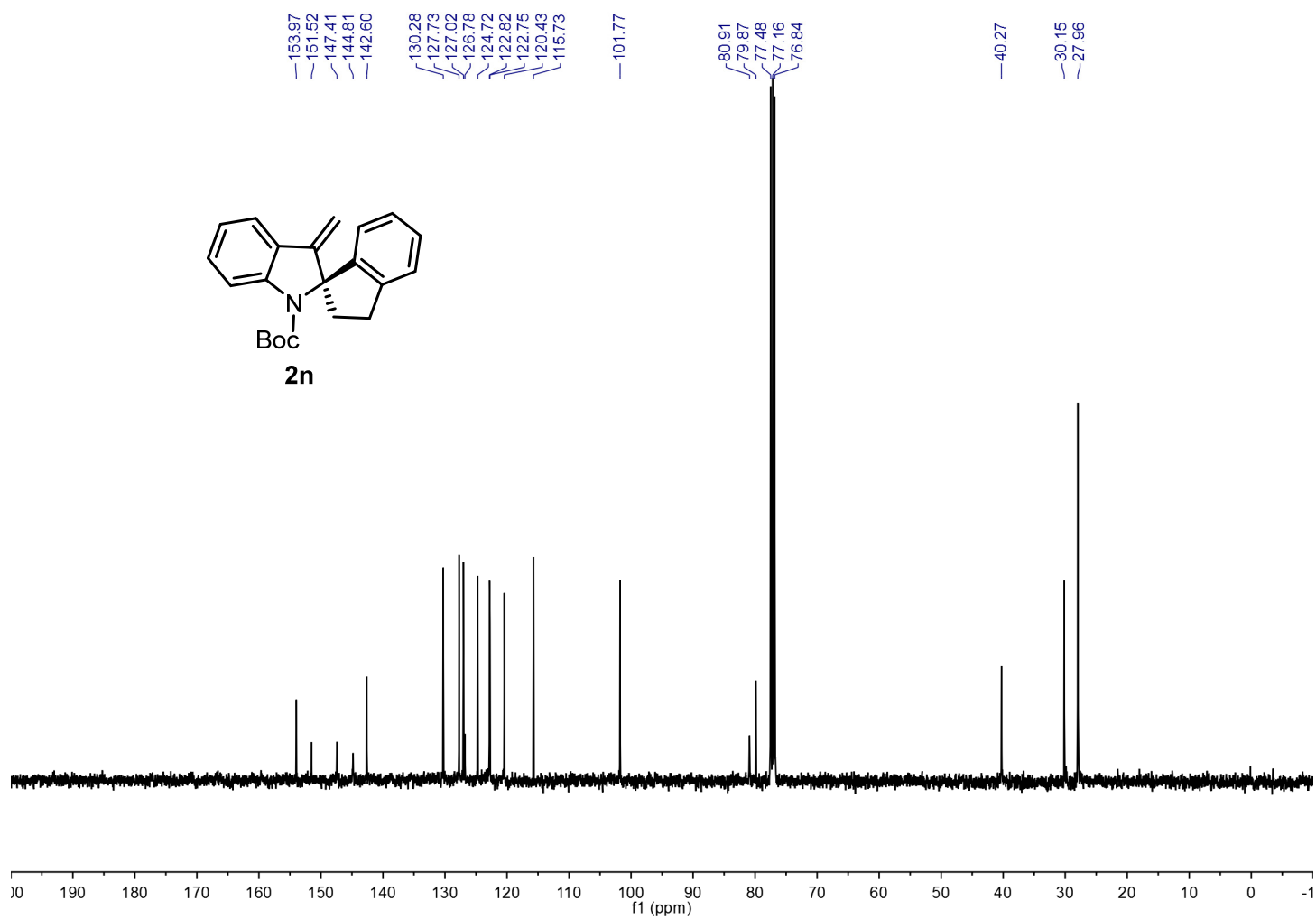
${ }^{1} \mathrm{H} \mathrm{NMR}\left(\mathrm{CDCl}_{3}, 400 \mathrm{MHz}\right)$ of $\mathbf{3 n a}$ :

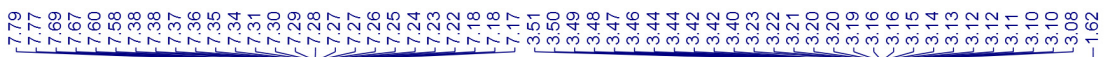
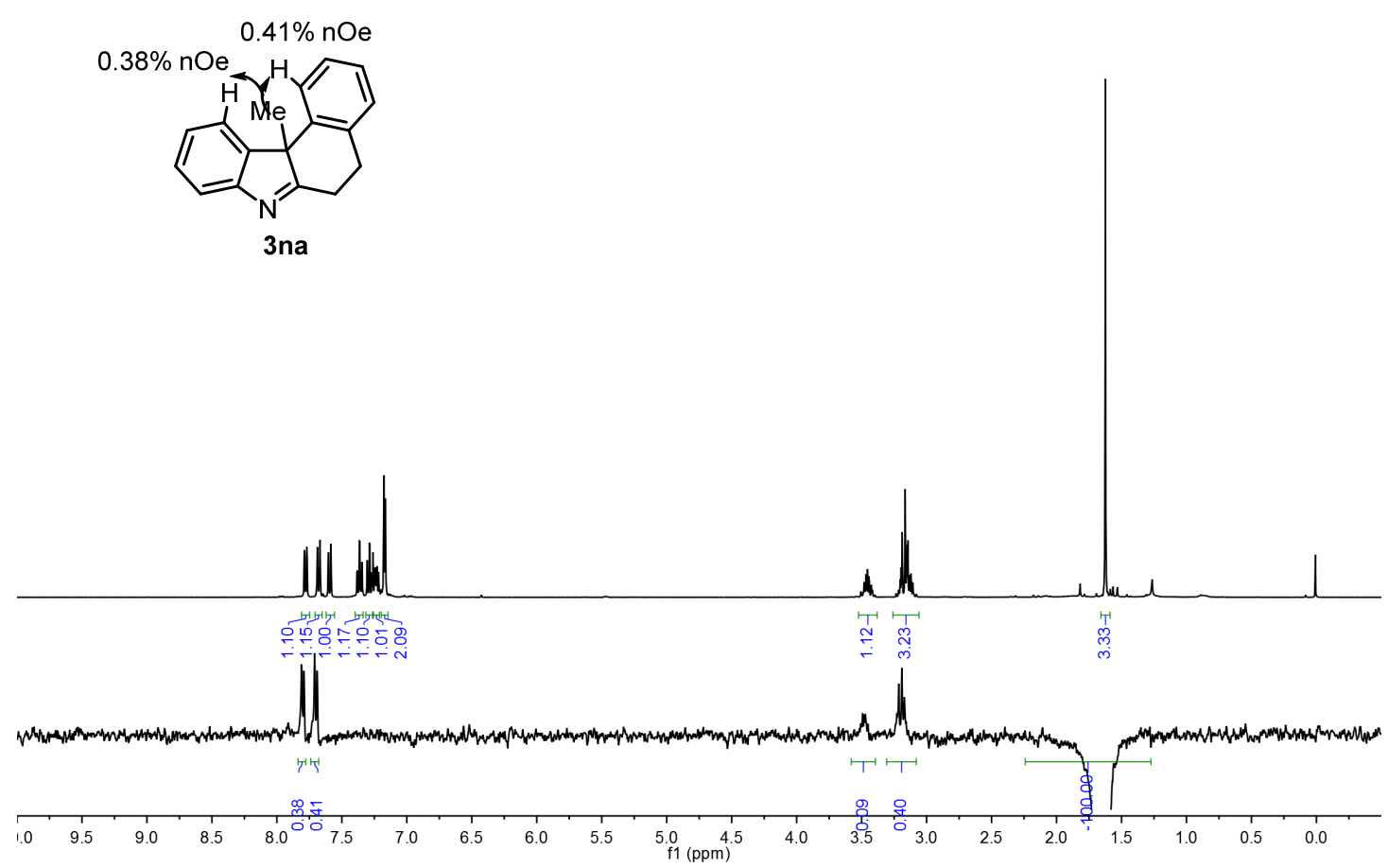

${ }^{13} \mathrm{C}\left\{{ }^{1} \mathrm{H}\right\}$ NMR $\left(\mathrm{CDCl}_{3}, 100 \mathrm{MHz}\right)$ of 3na:
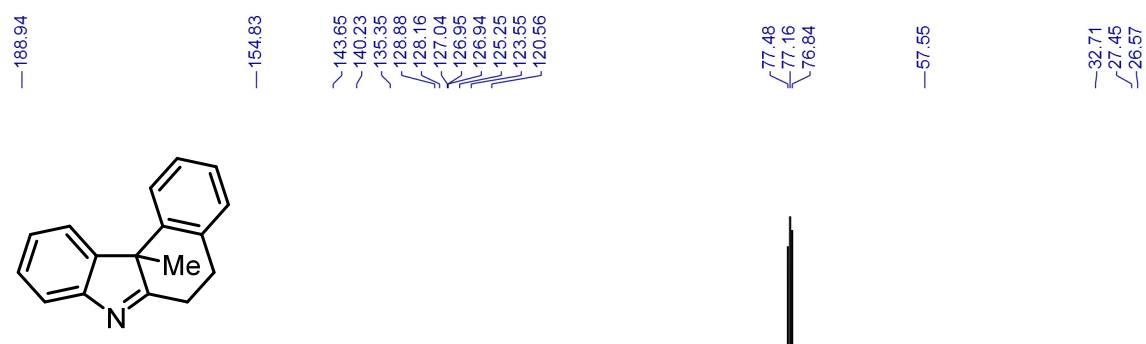

3na
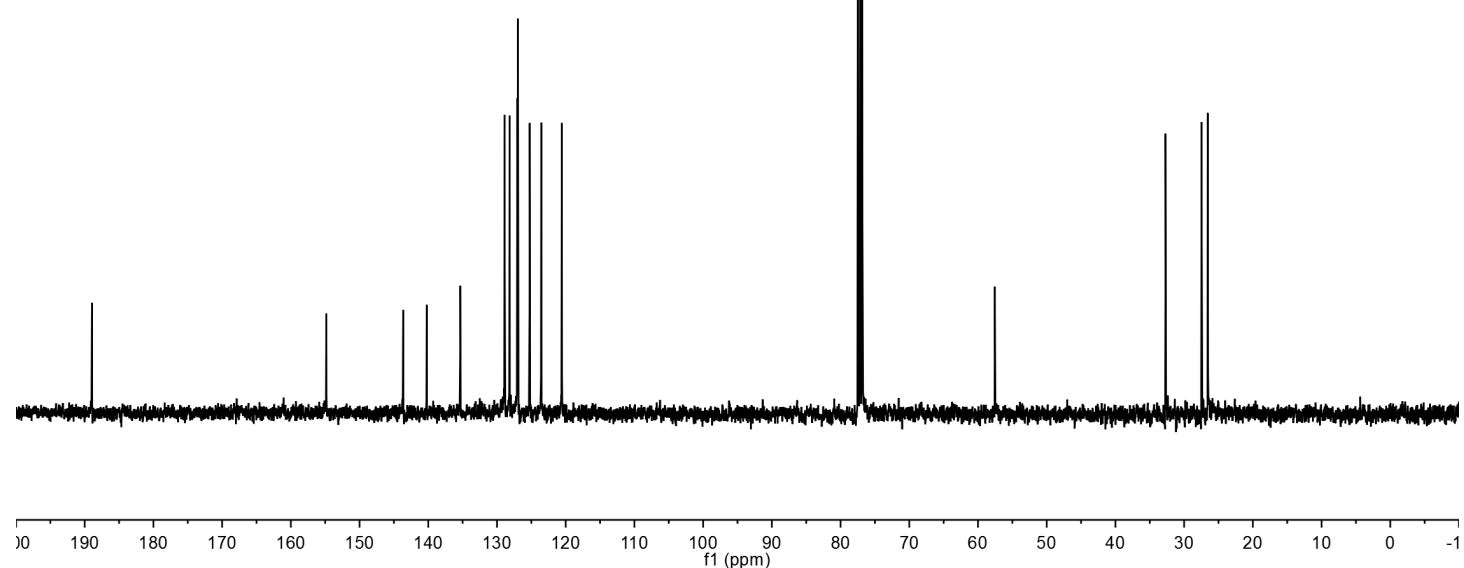
2D COSY of 3na:

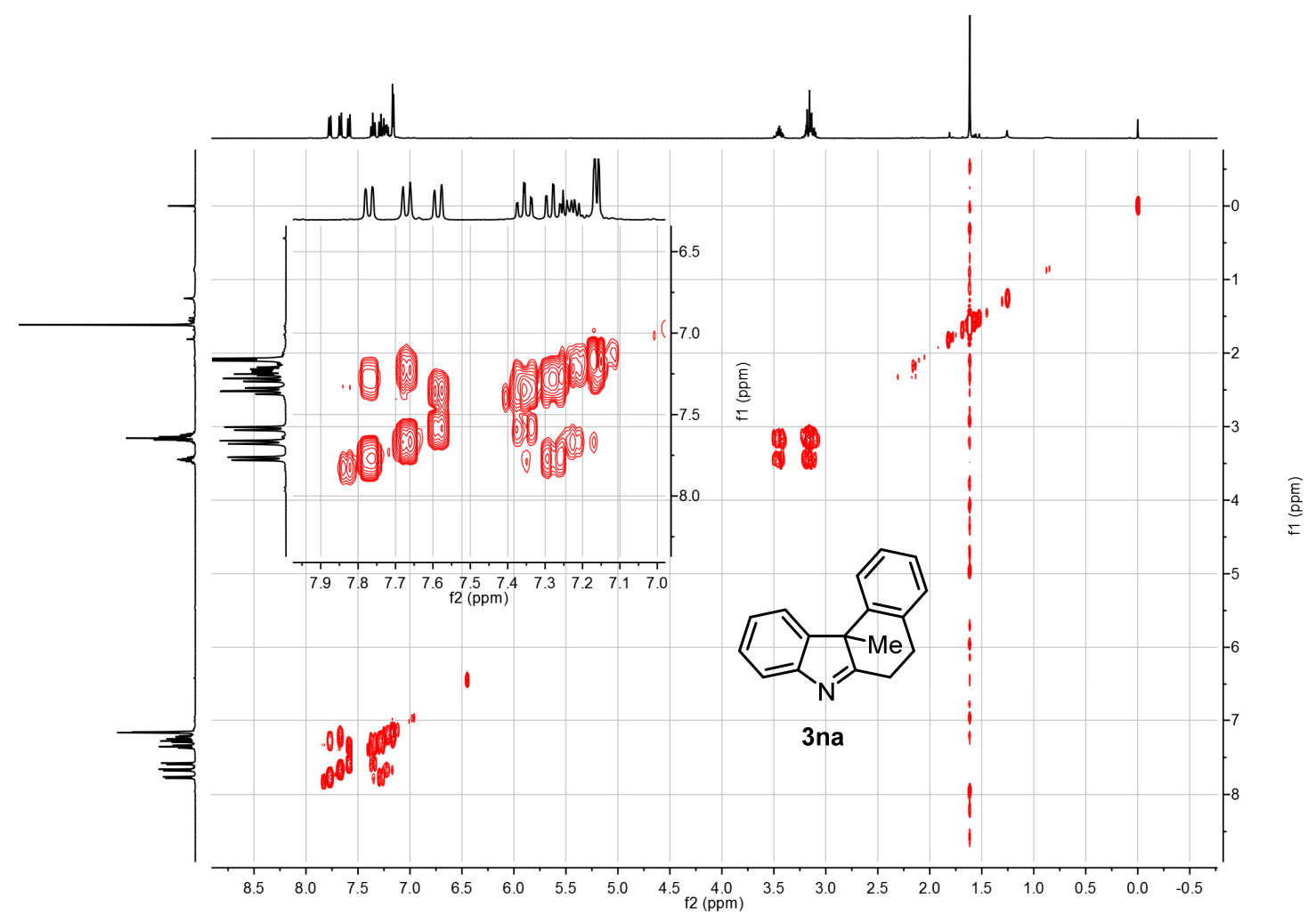

2D HSQC of 3na:

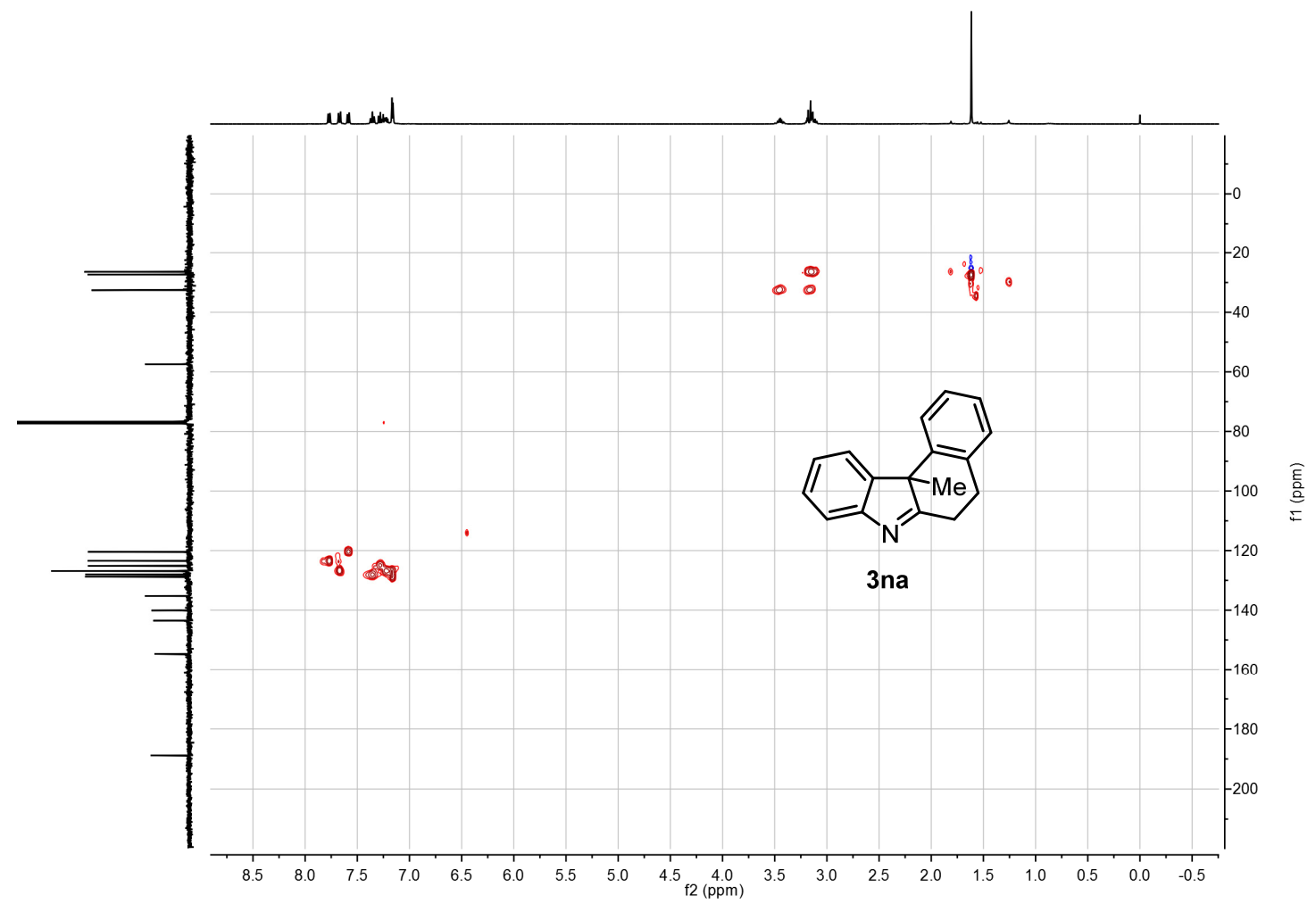


2D HMBC of 3na:

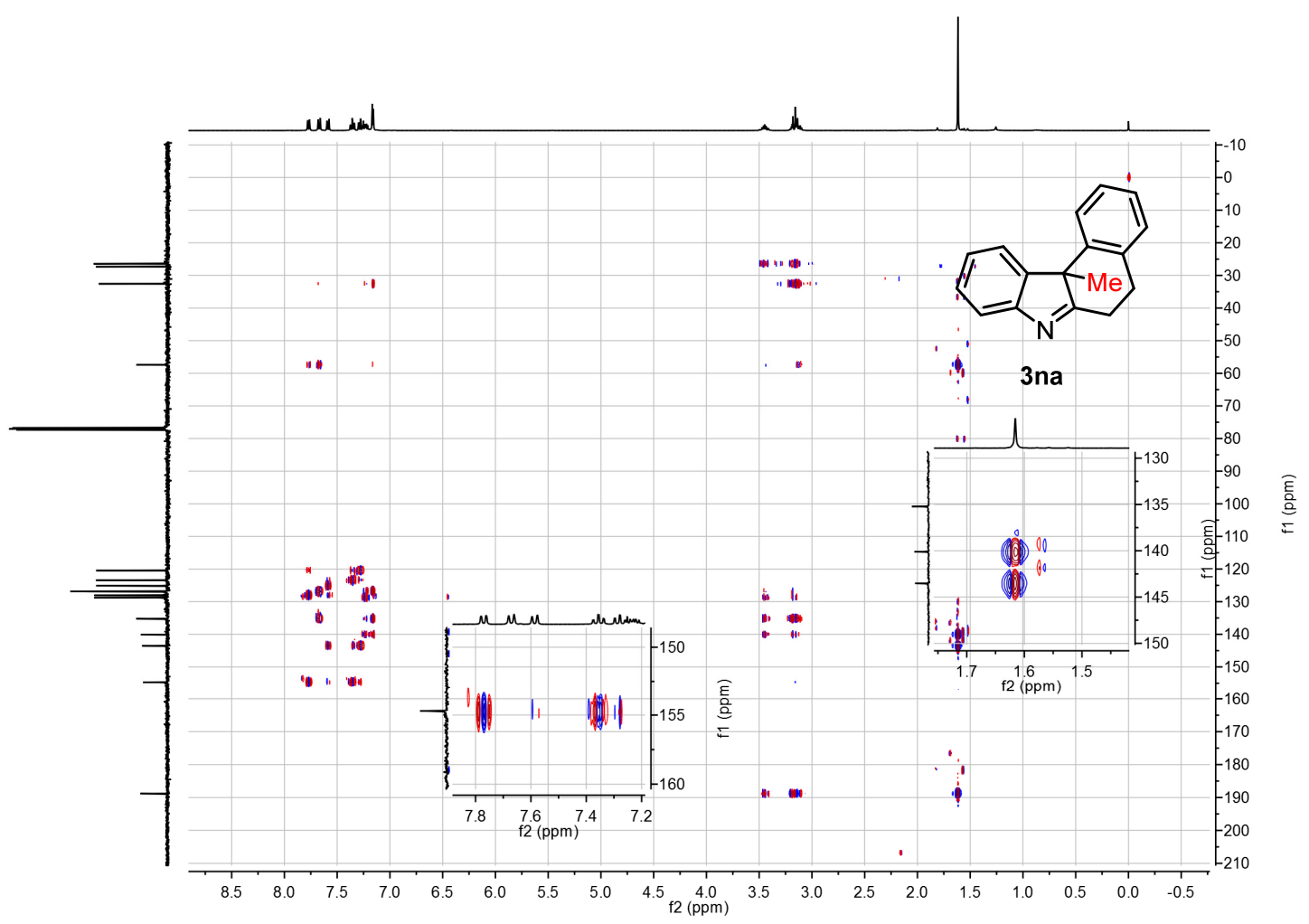


${ }^{1} \mathrm{H}$ NMR $\left(\mathrm{CDCl}_{3}, 400 \mathrm{MHz}\right)$ of $\mathbf{3 n b}$ :

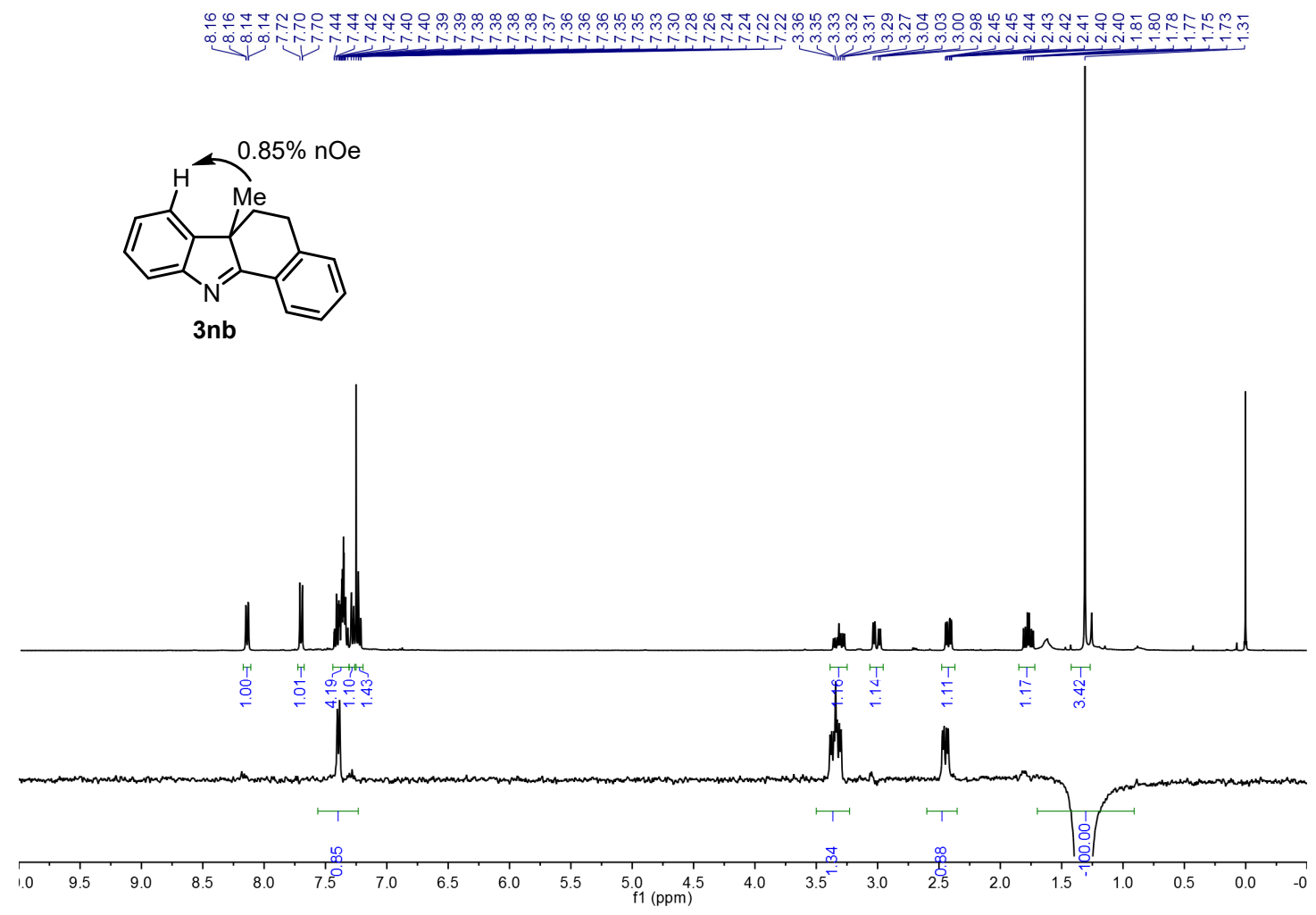

${ }^{13} \mathrm{C}\left\{{ }^{1} \mathrm{H}\right\}$ NMR $\left(\mathrm{CDCl}_{3}, 100 \mathrm{MHz}\right)$ of $\mathbf{3 n b}$ :
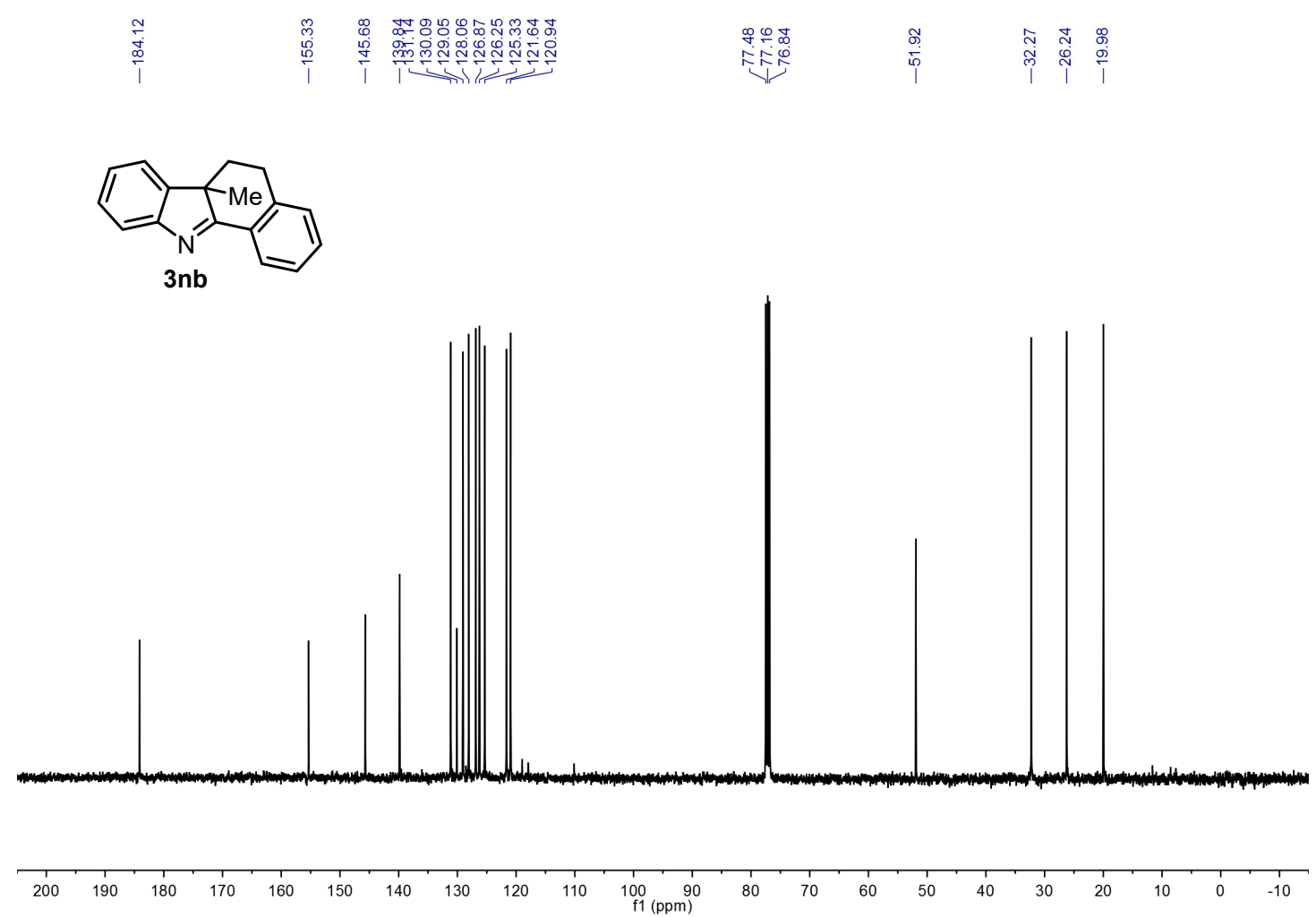
2D COSY of $\mathbf{3 n b}$

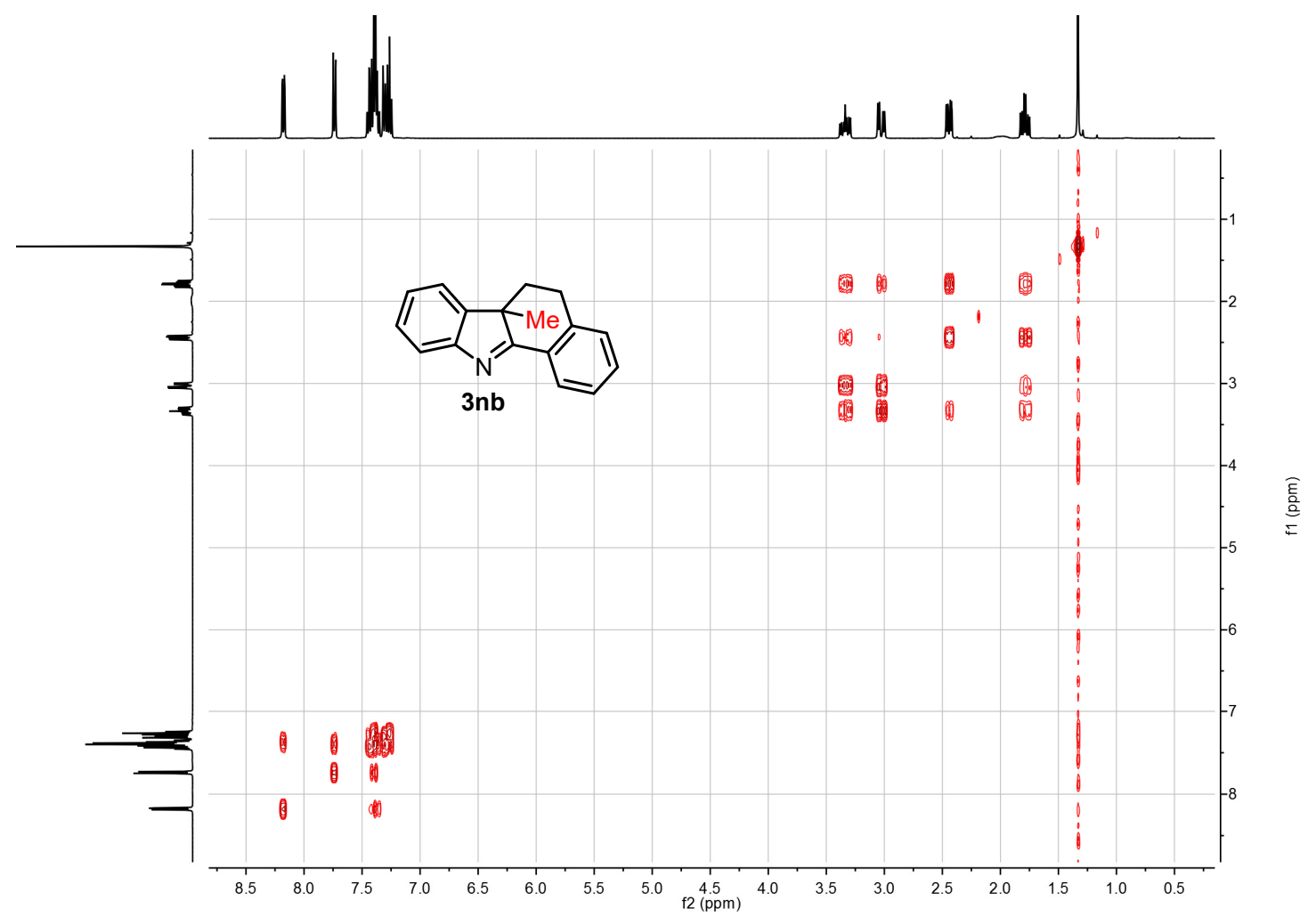

2D HSQC of 3nb:

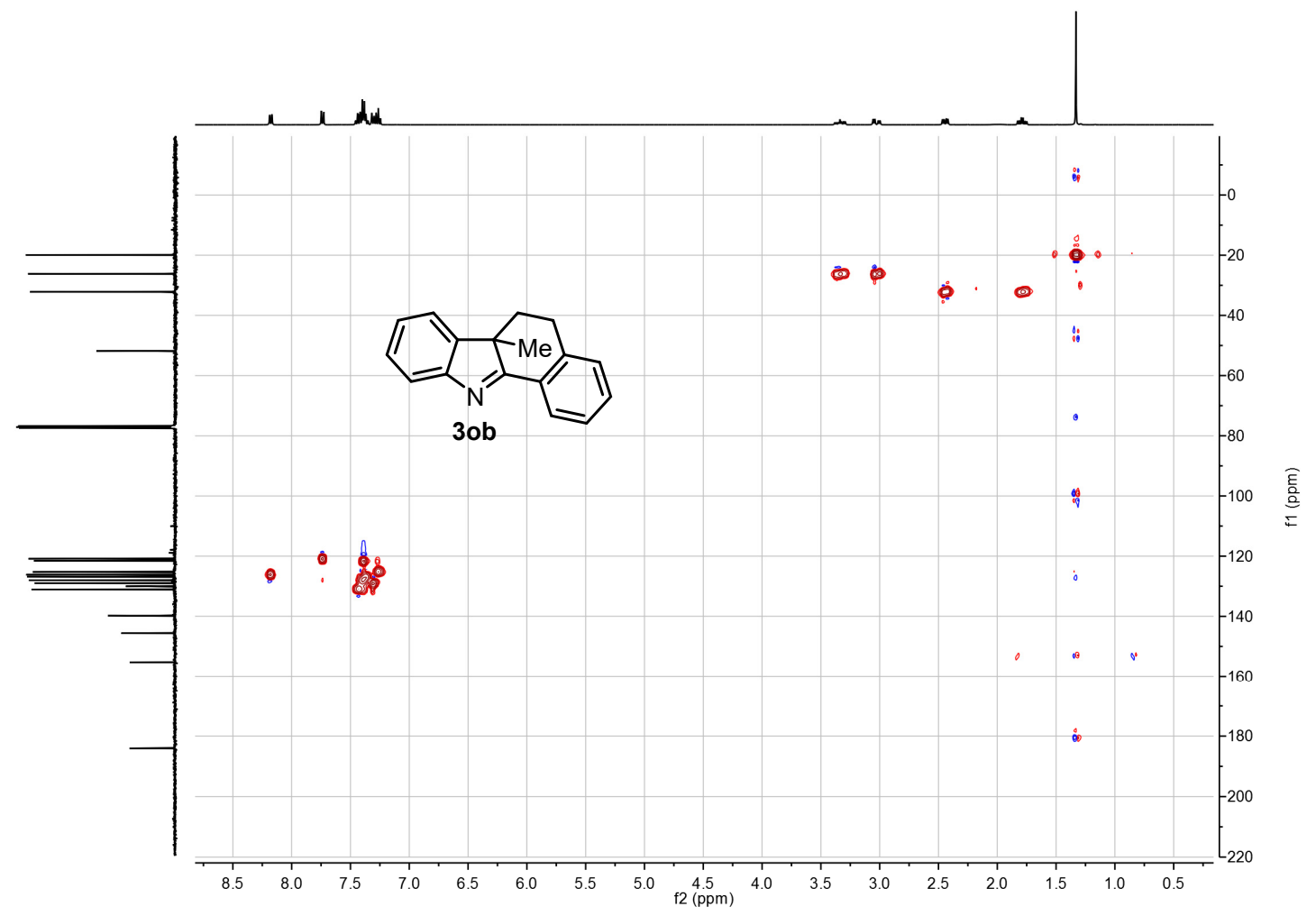

S85 
2D HMBC of $\mathbf{3 n b}$ :

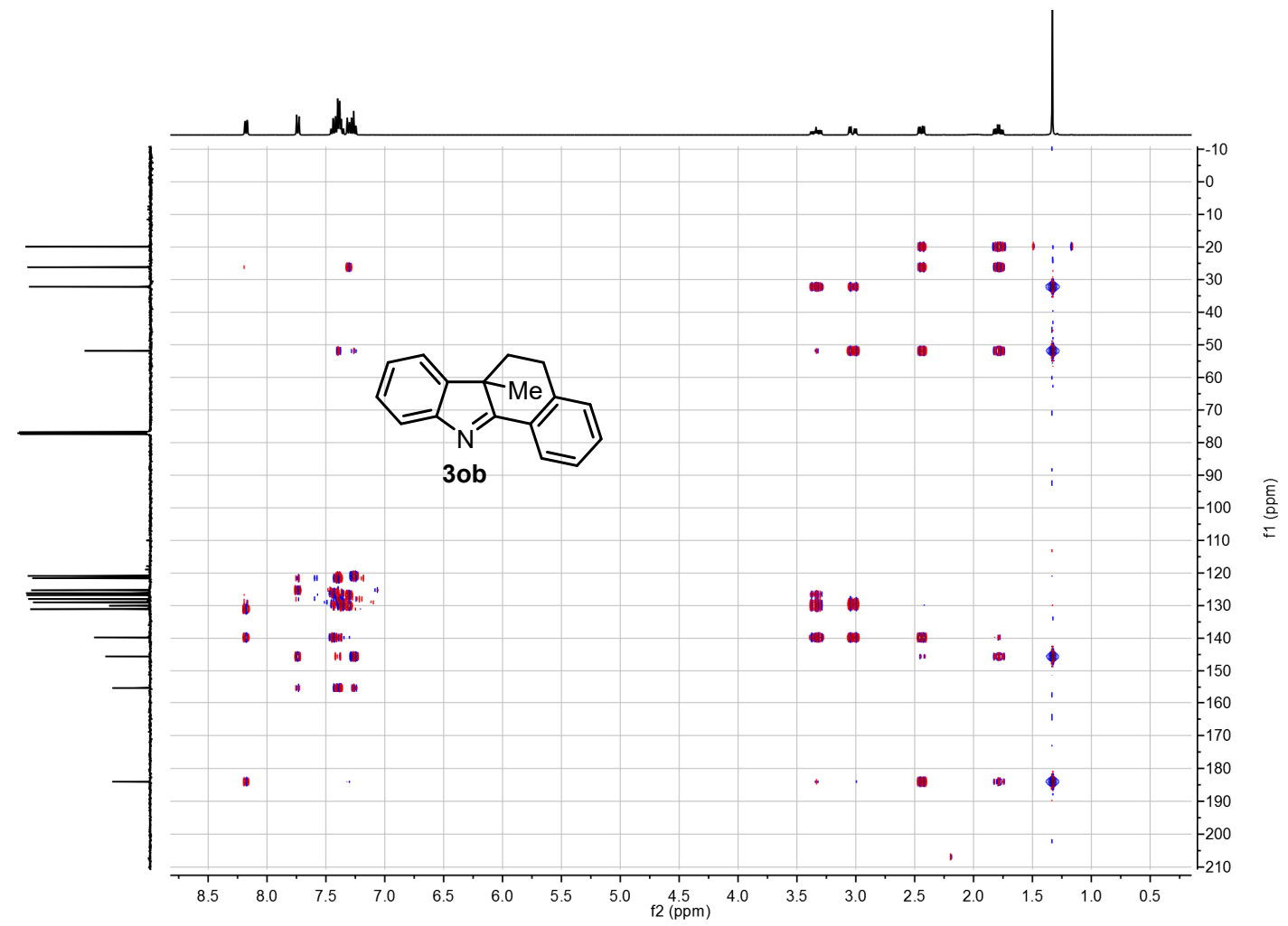


${ }^{1} \mathrm{H}$ NMR $\left(\mathrm{CDCl}_{3}, 400 \mathrm{MHz}\right)$ of $\mathbf{2 o}$ :

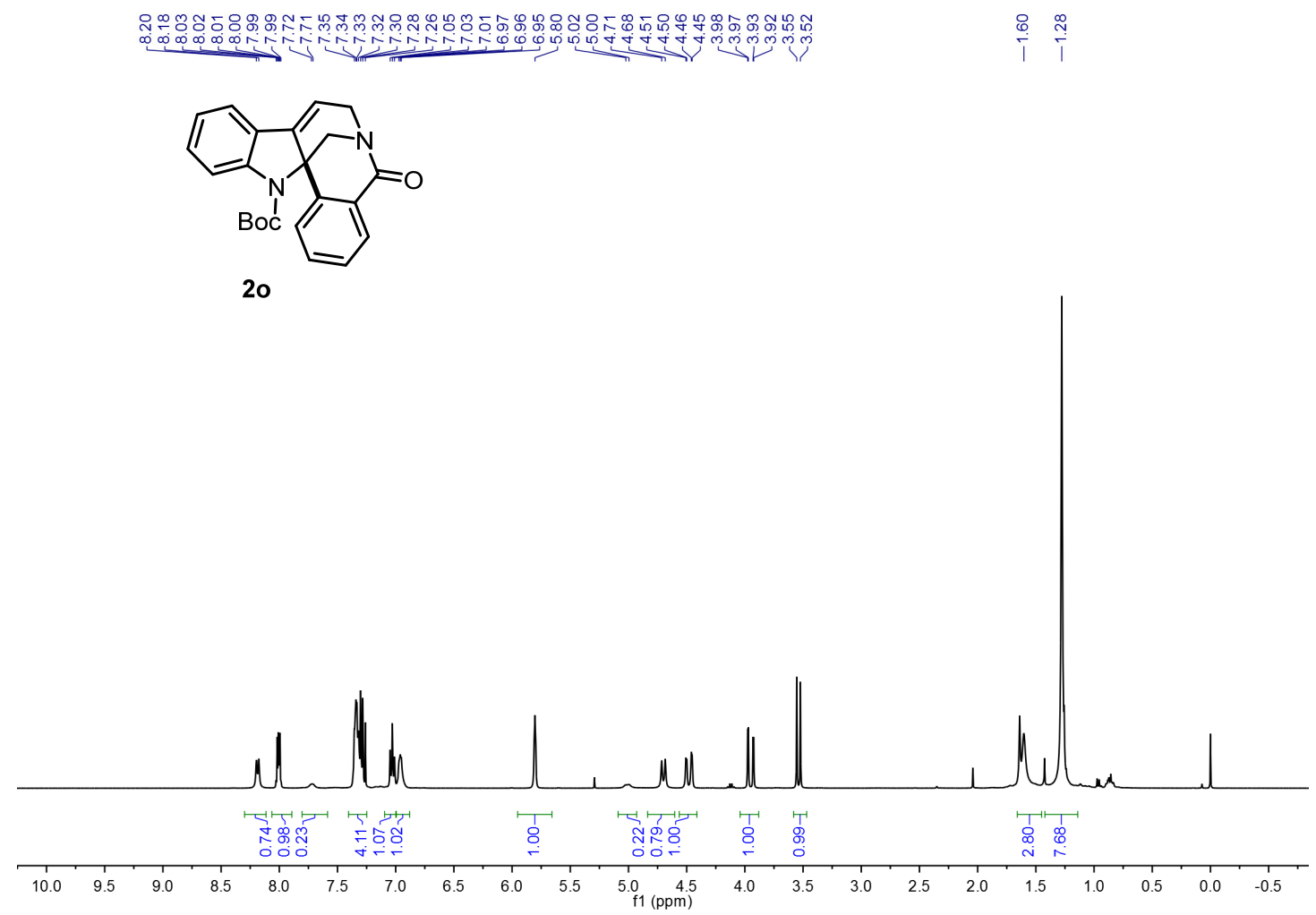

${ }^{13} \mathrm{C}\left\{{ }^{1} \mathrm{H}\right\}$ NMR $\left(\mathrm{CDCl}_{3}, 100 \mathrm{MHz}\right)$ of $\mathbf{2 o}$ :
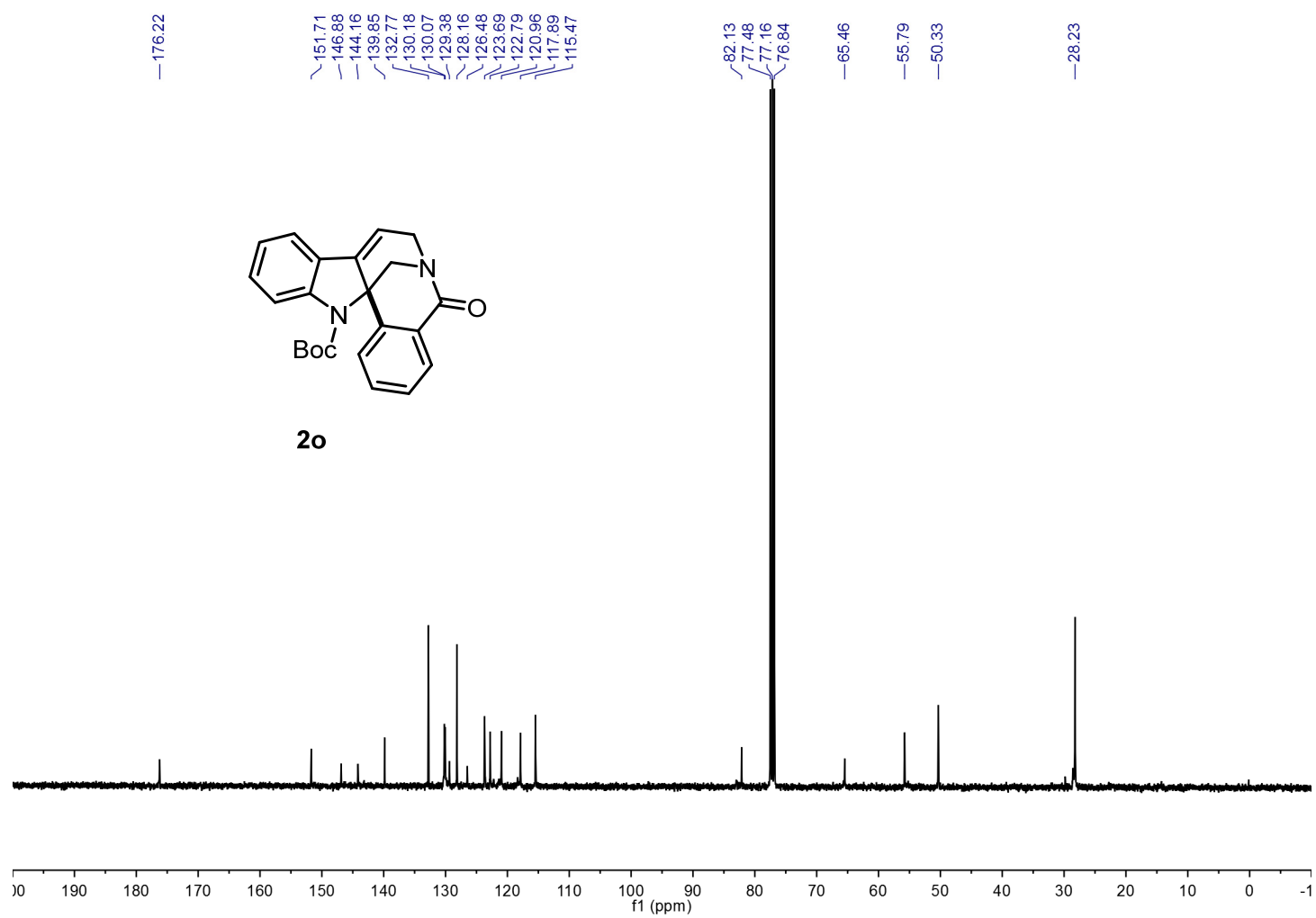
${ }^{1} \mathrm{H}$ NMR $\left(\mathrm{CDCl}_{3}, 400 \mathrm{MHz}\right)$ of $\mathbf{4 h a}:$

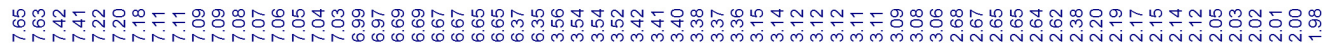

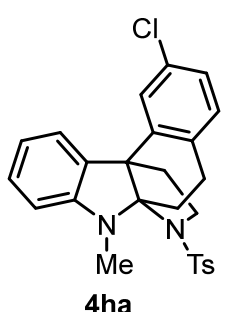

4ha

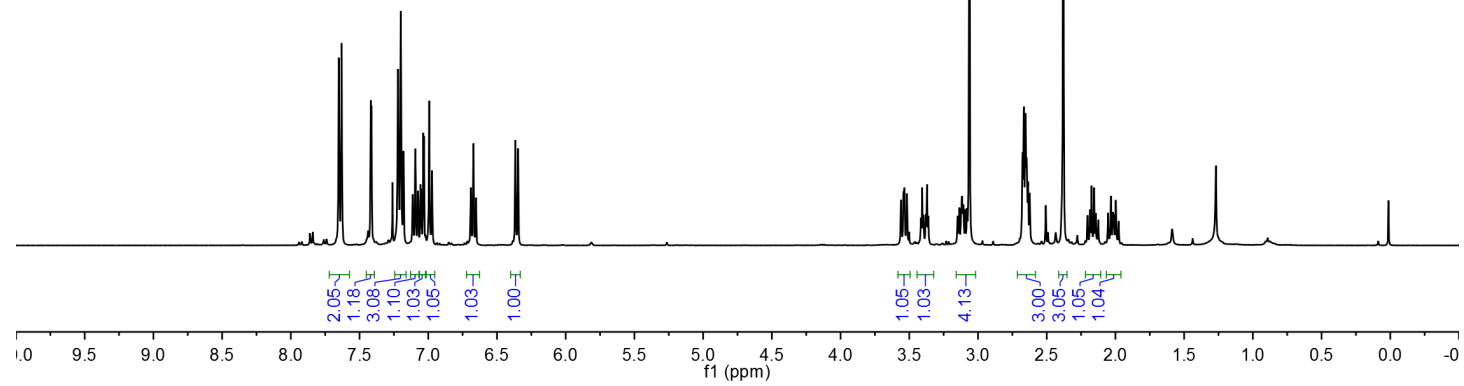

${ }^{13} \mathrm{C}\left\{{ }^{1} \mathrm{H}\right\} \mathrm{NMR}\left(\mathrm{CDCl}_{3}, 150 \mathrm{MHz}\right)$ of 4 ha:
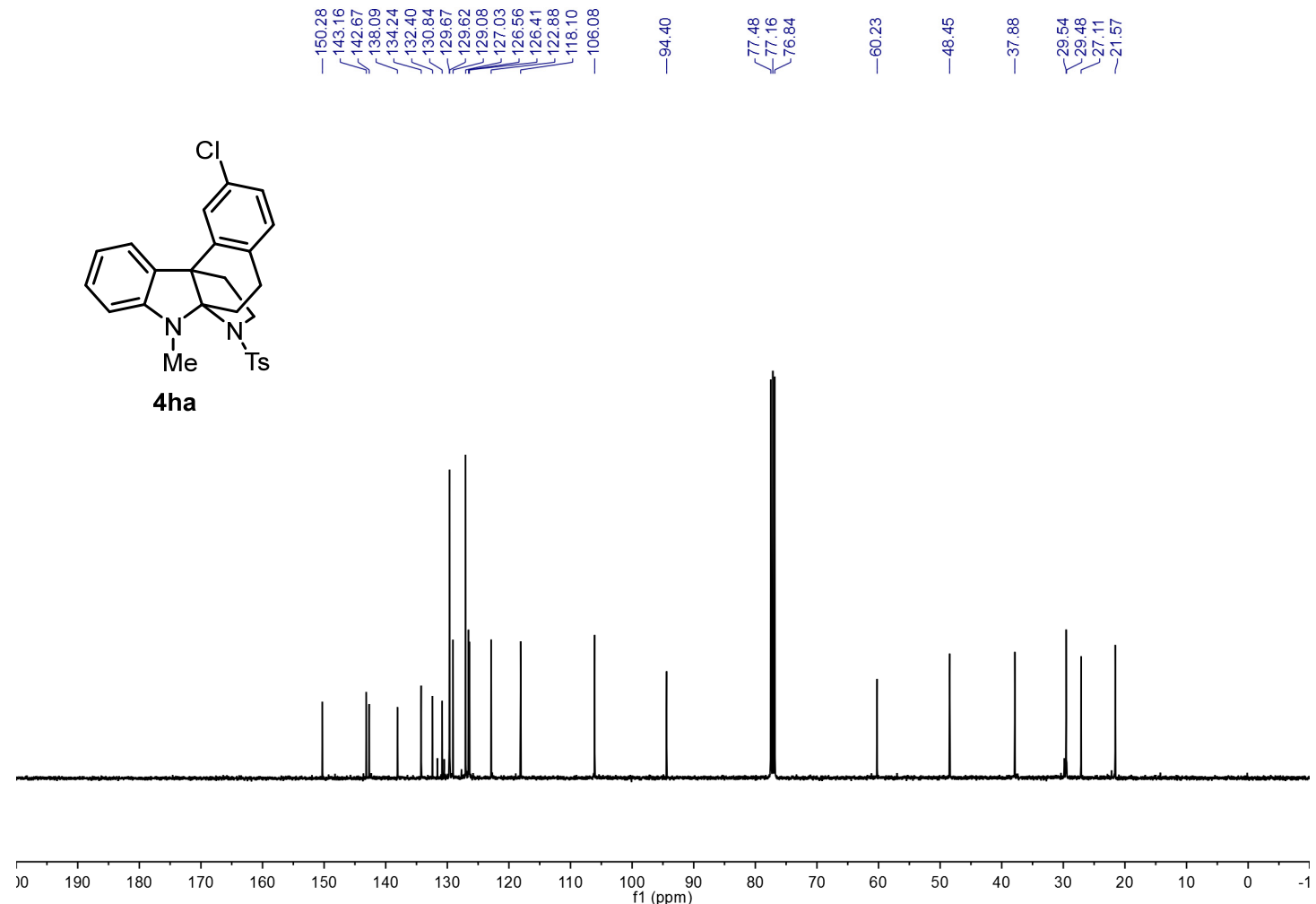
${ }^{1} \mathrm{H} \mathrm{NMR}\left(\mathrm{CDCl}_{3}, 400 \mathrm{MHz}\right)$ of $\mathbf{4 h b}$ :

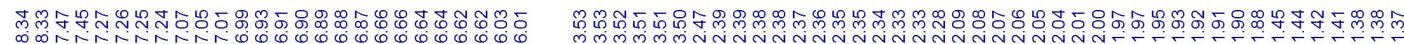
पing

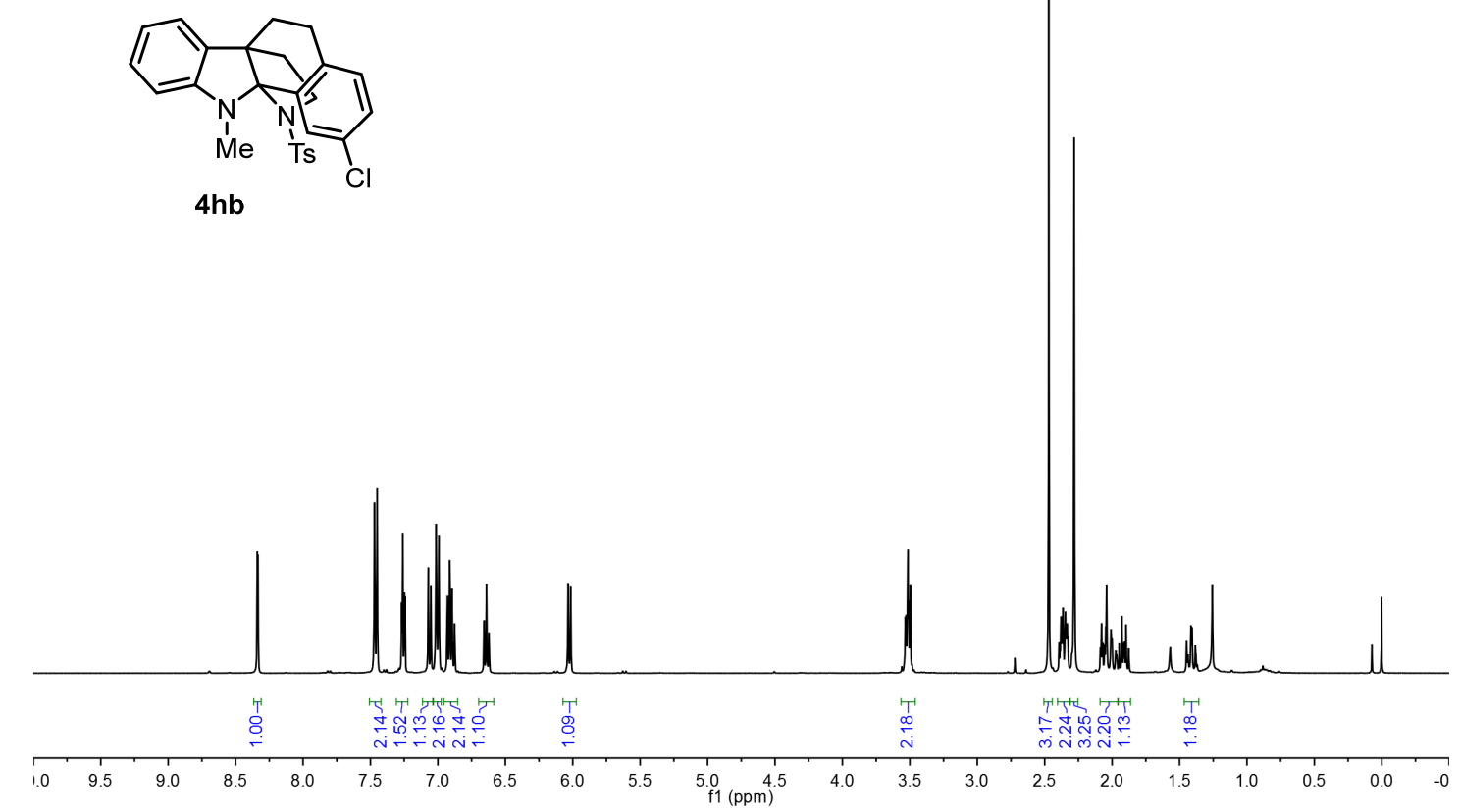

${ }^{13} \mathrm{C}\left\{{ }^{1} \mathrm{H}\right\}$ NMR $\left(\mathrm{CDCl}_{3}, 150 \mathrm{MHz}\right)$ of $\mathbf{4 h \mathbf { b }}$ :

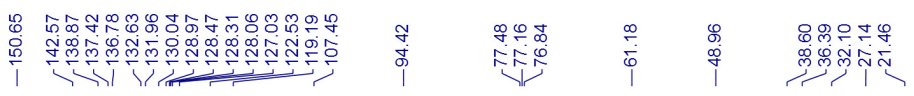
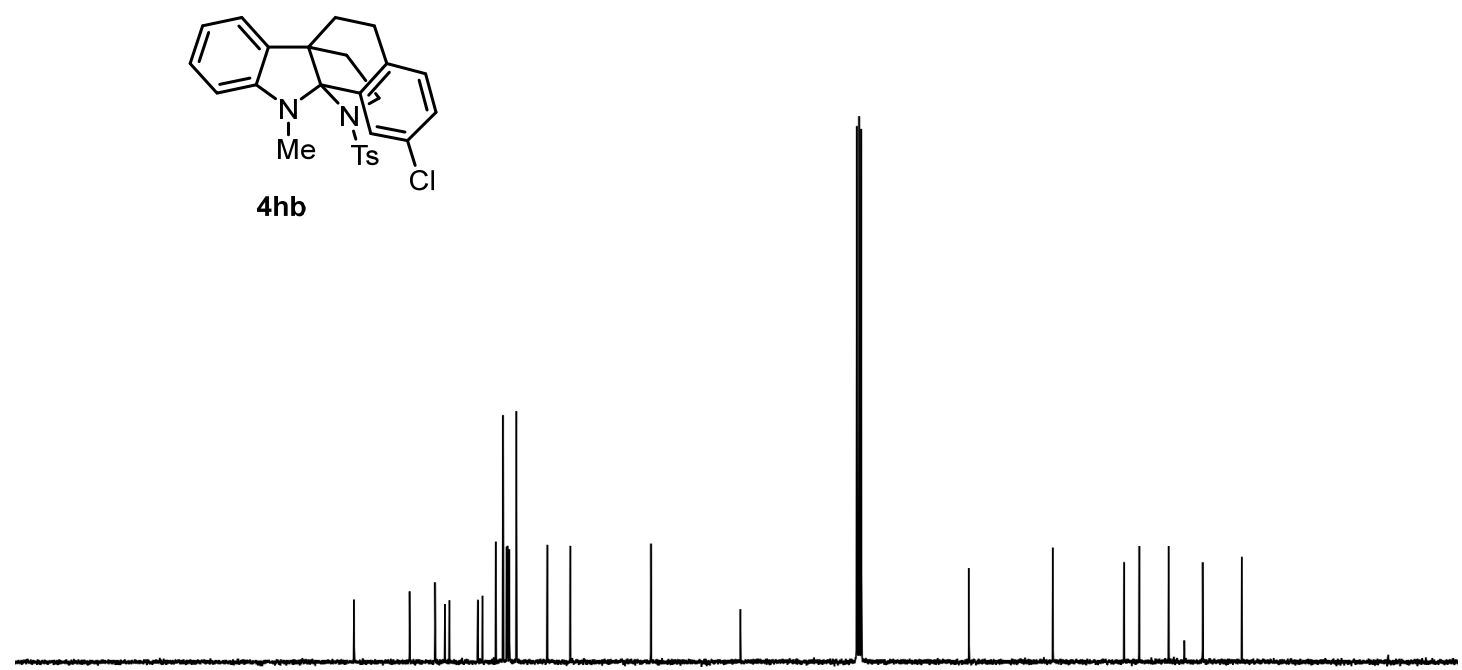

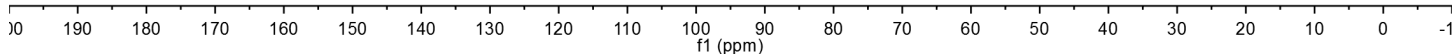


${ }^{1} \mathrm{H} \mathrm{NMR}\left(\mathrm{CDCl}_{3}, 400 \mathrm{MHz}\right)$ of 4:

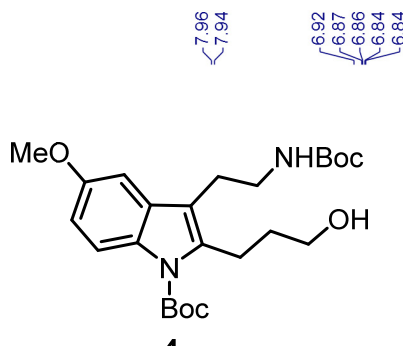

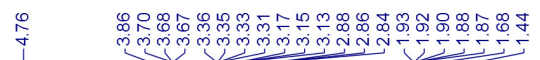

4
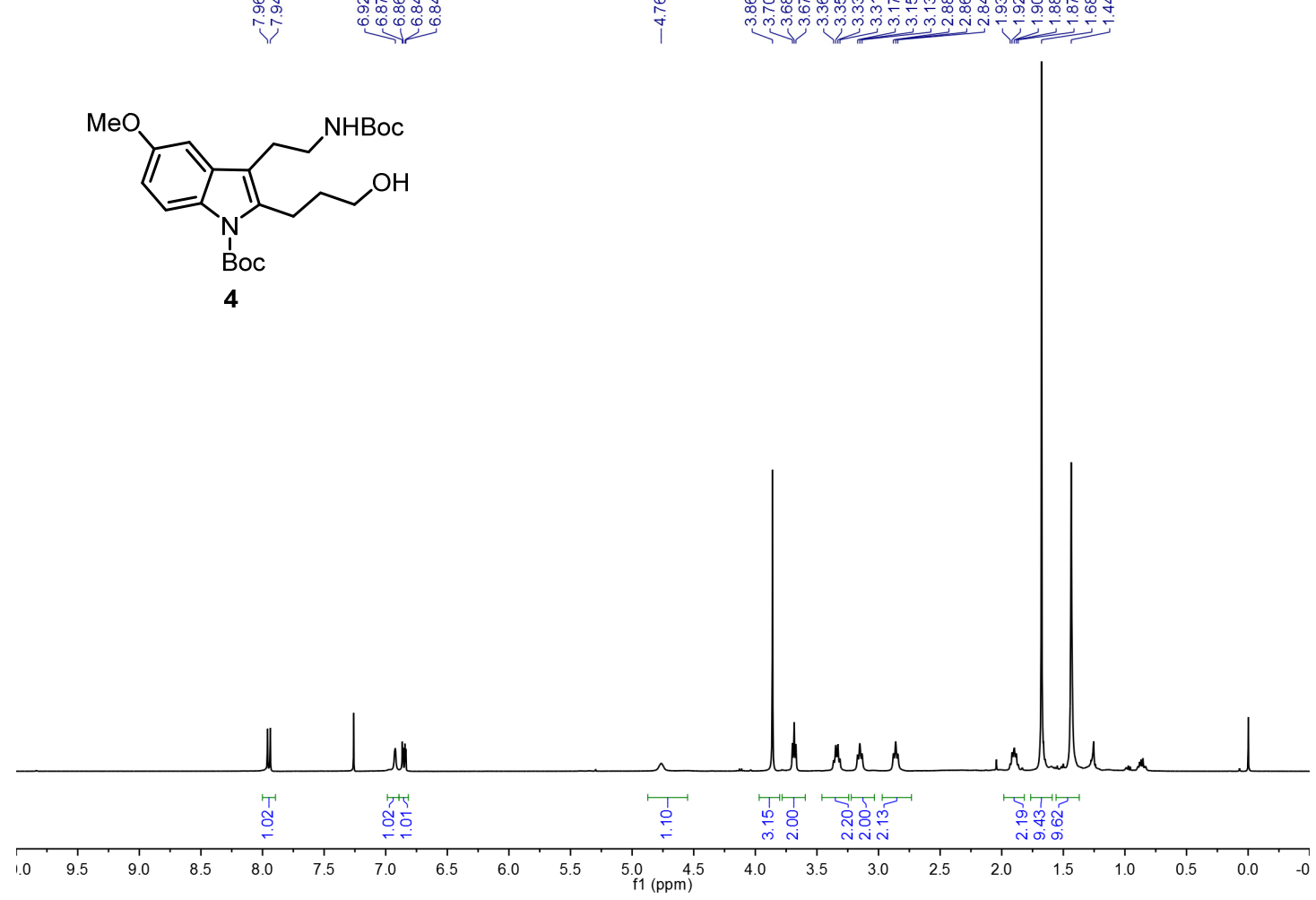

${ }^{13} \mathrm{C}\left\{{ }^{1} \mathrm{H}\right\}$ NMR $\left(\mathrm{CDCl}_{3}, 100 \mathrm{MHz}\right)$ of $\mathbf{4}$ :
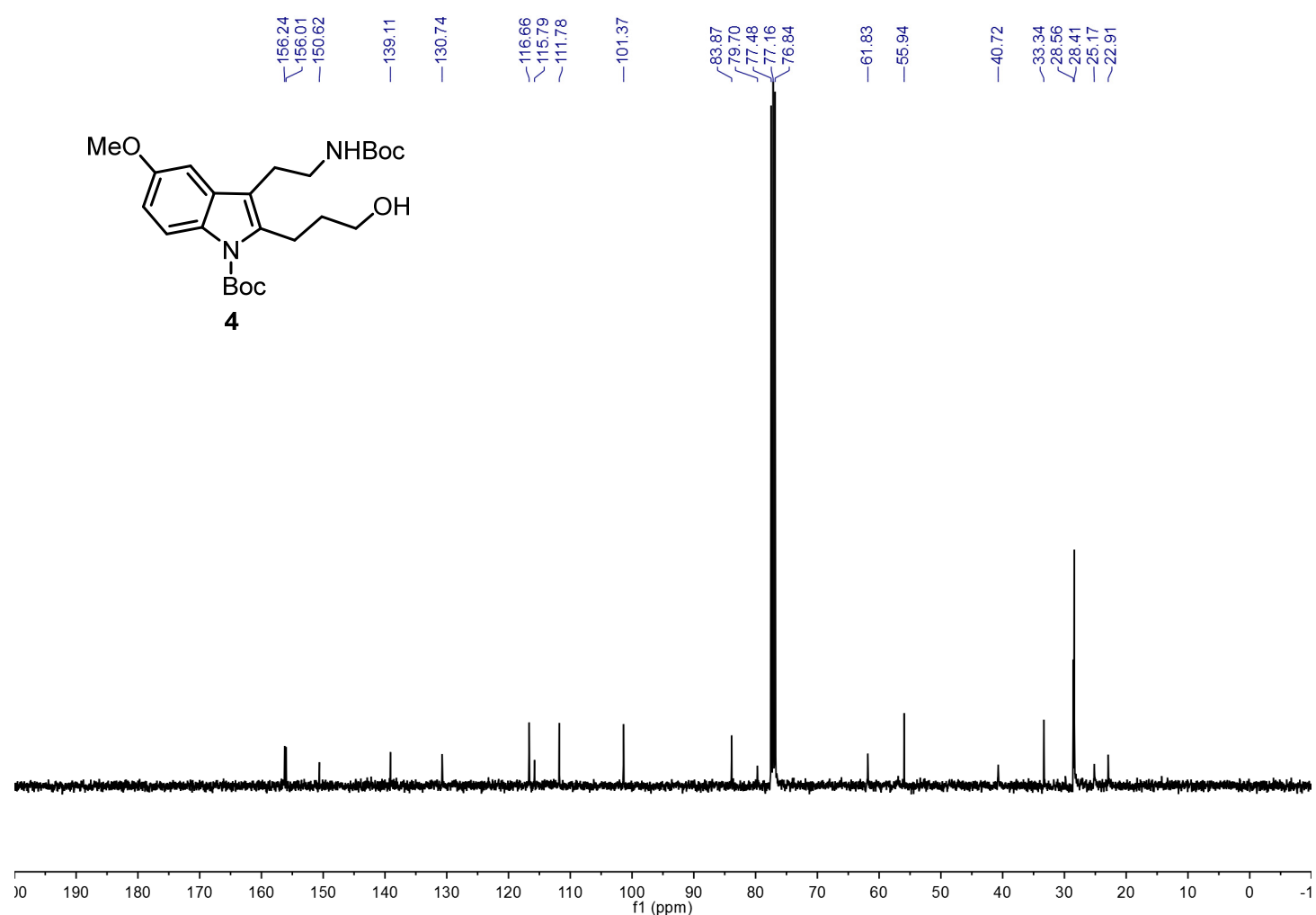
${ }^{1} \mathrm{H}$ NMR $\left(\mathrm{CDCl}_{3}, 400 \mathrm{MHz}\right)$ of $\mathbf{6}$ :

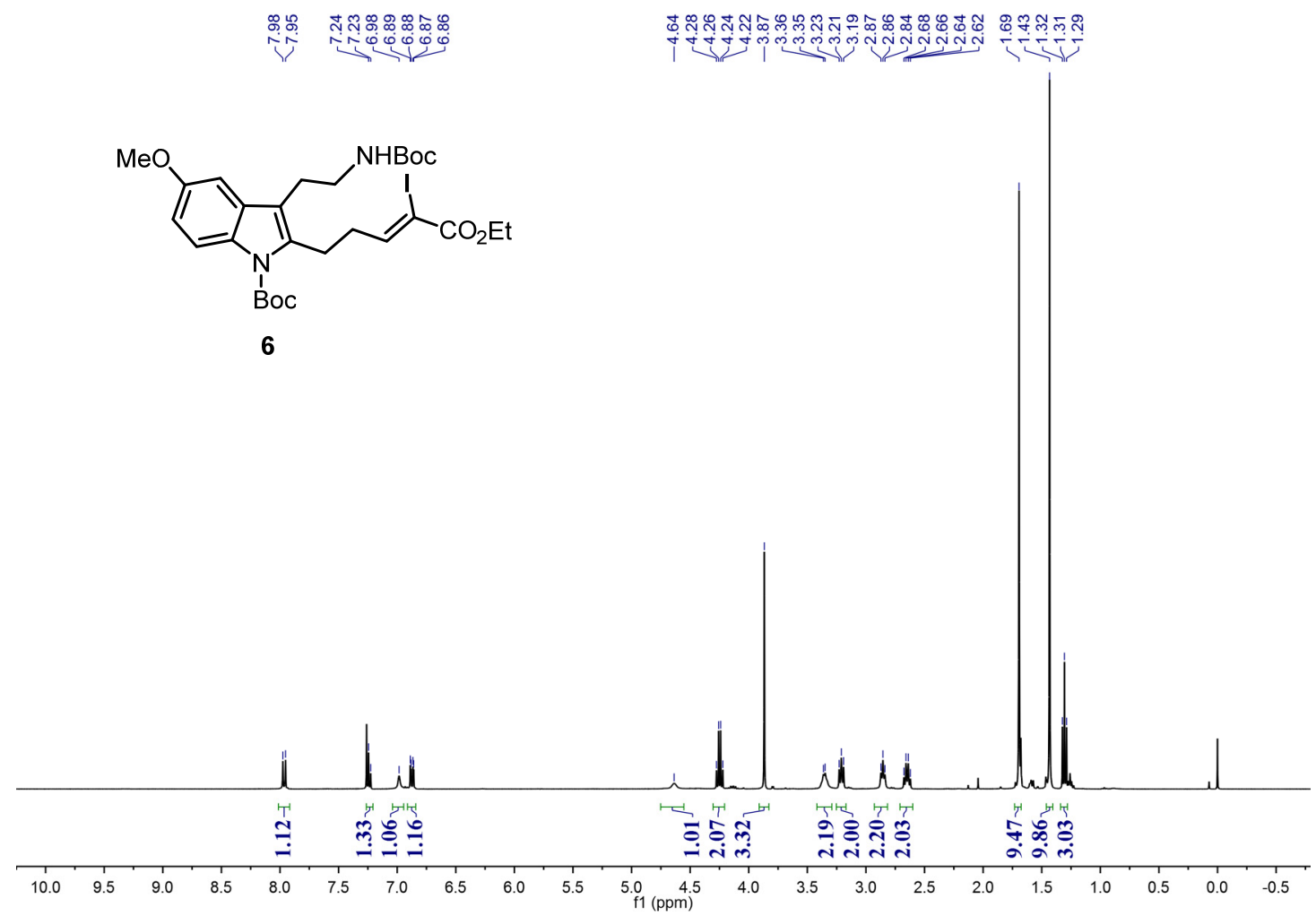

${ }^{13} \mathrm{C}\left\{{ }^{1} \mathrm{H}\right\}$ NMR $\left(\mathrm{CDCl}_{3}, 100 \mathrm{MHz}\right)$ of $\mathbf{6}$ :

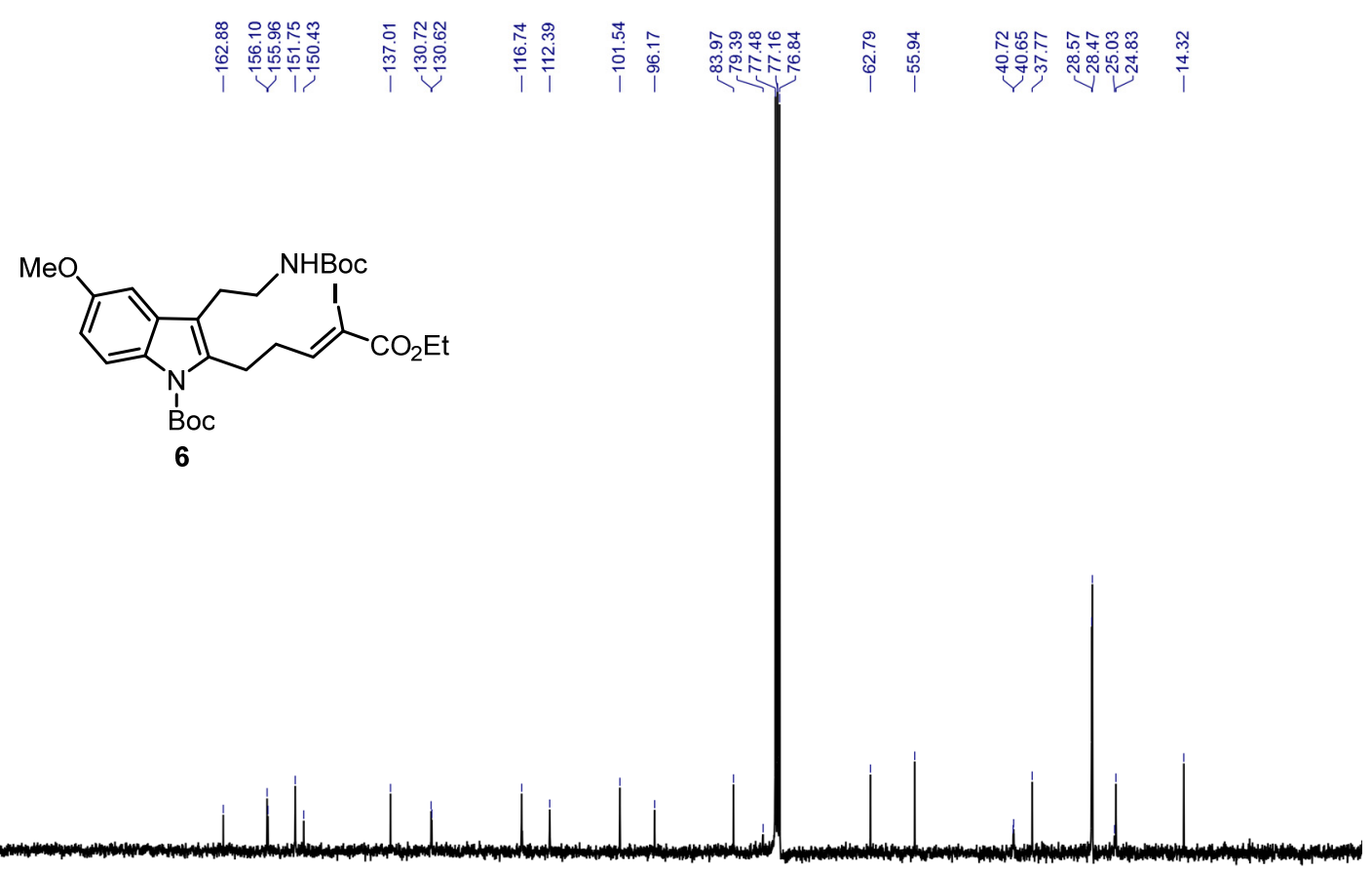

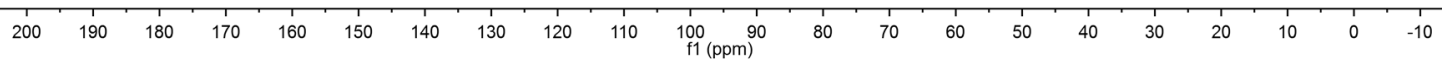


${ }^{1} \mathrm{H}$ NMR $\left(\mathrm{CDCl}_{3}, 400 \mathrm{MHz}\right)$ of 7:

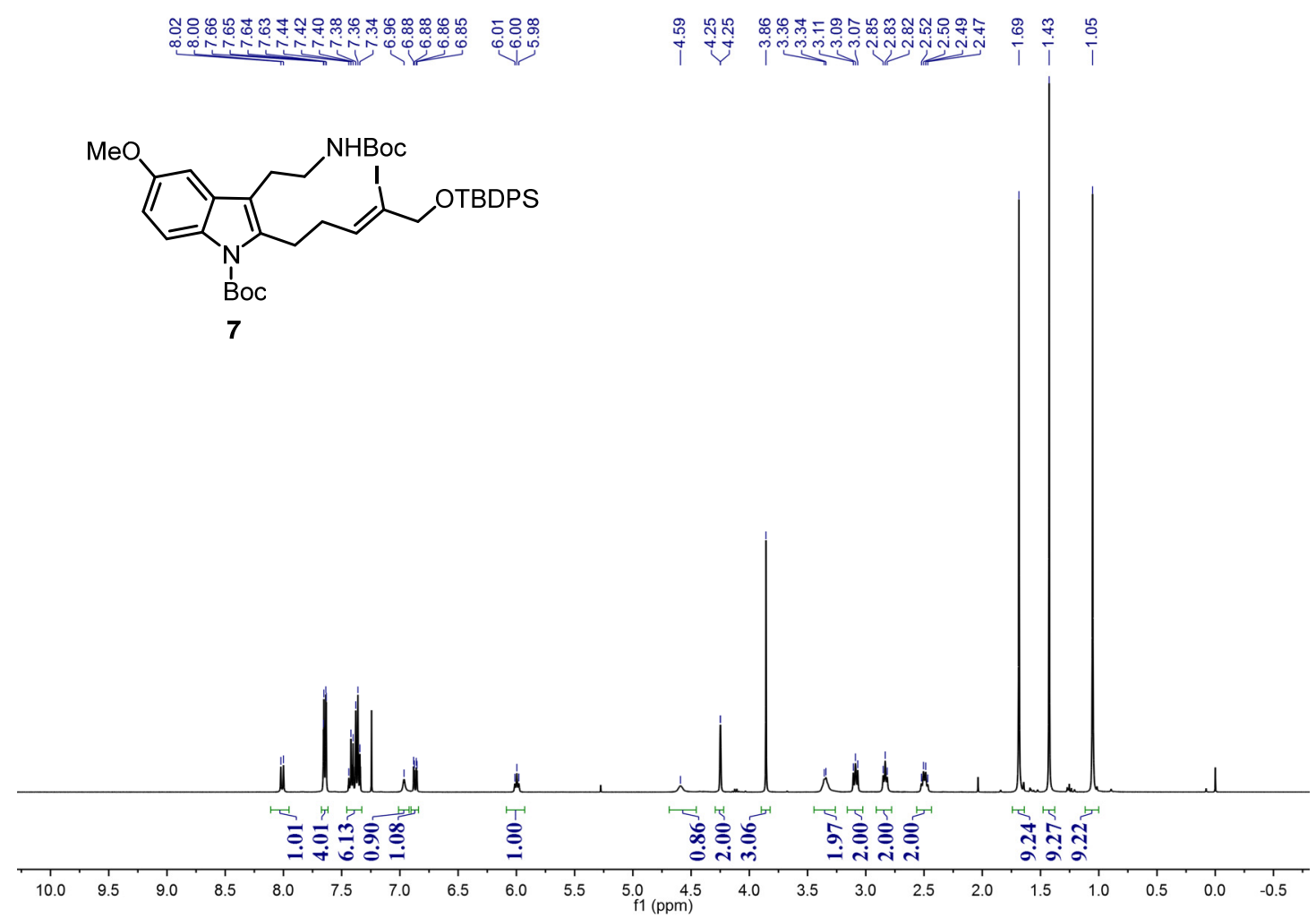

${ }^{13} \mathrm{C}\left\{{ }^{1} \mathrm{H}\right\} \mathrm{NMR}\left(\mathrm{CDCl}_{3}, 100 \mathrm{MHz}\right)$ of 7 :

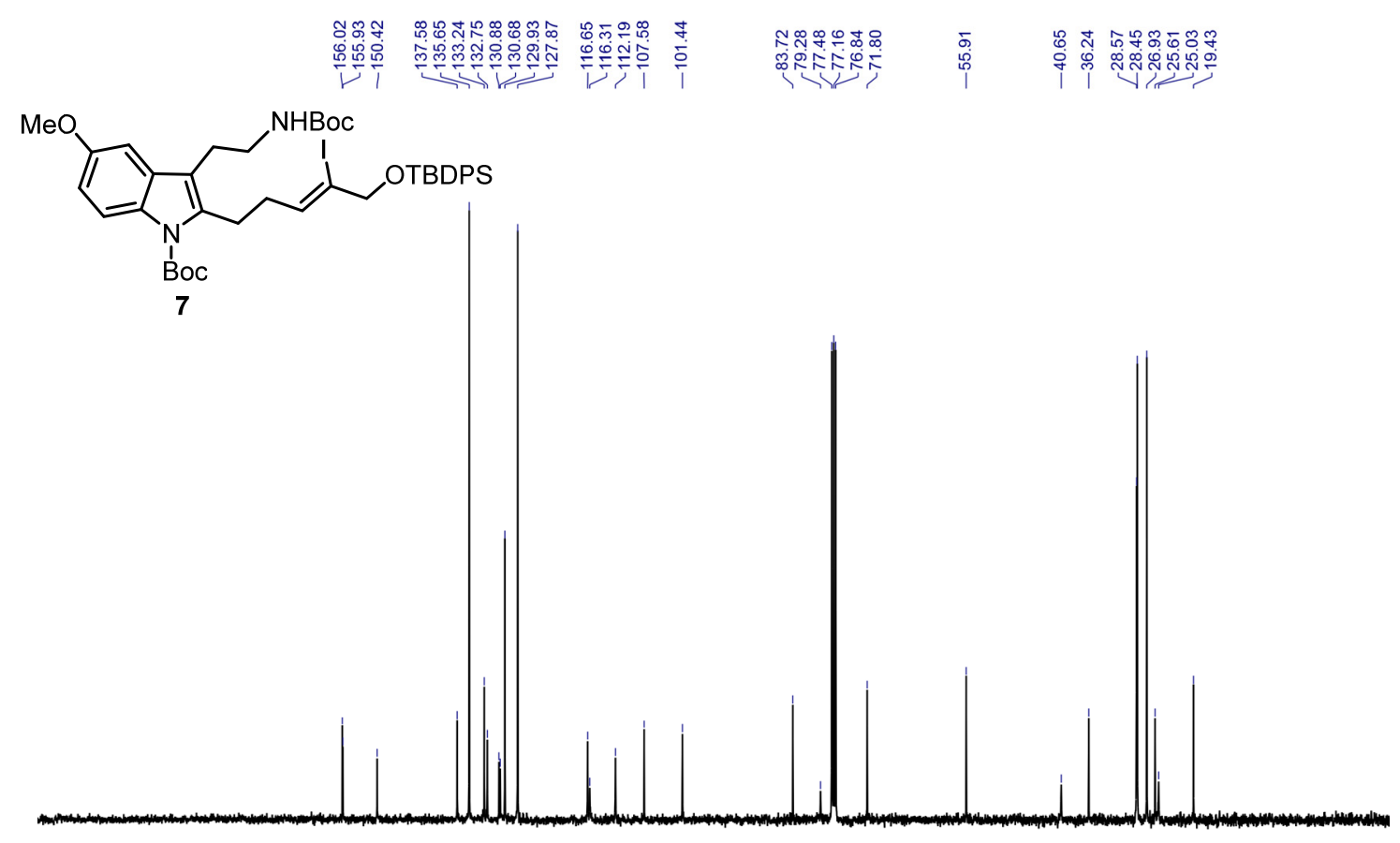

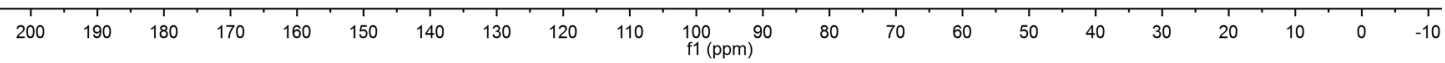


${ }^{1} \mathrm{H} \mathrm{NMR}\left(\mathrm{CDCl}_{3}, 400 \mathrm{MHz}\right)$ of $\mathbf{9}$ :

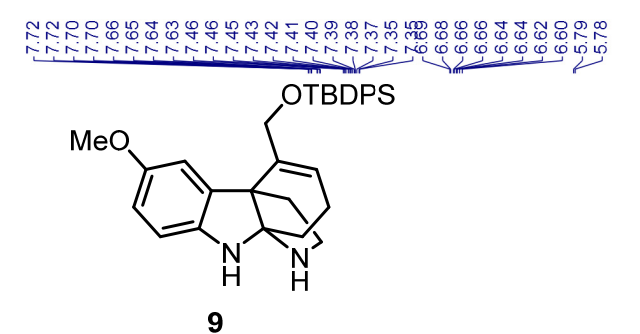

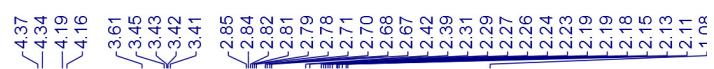

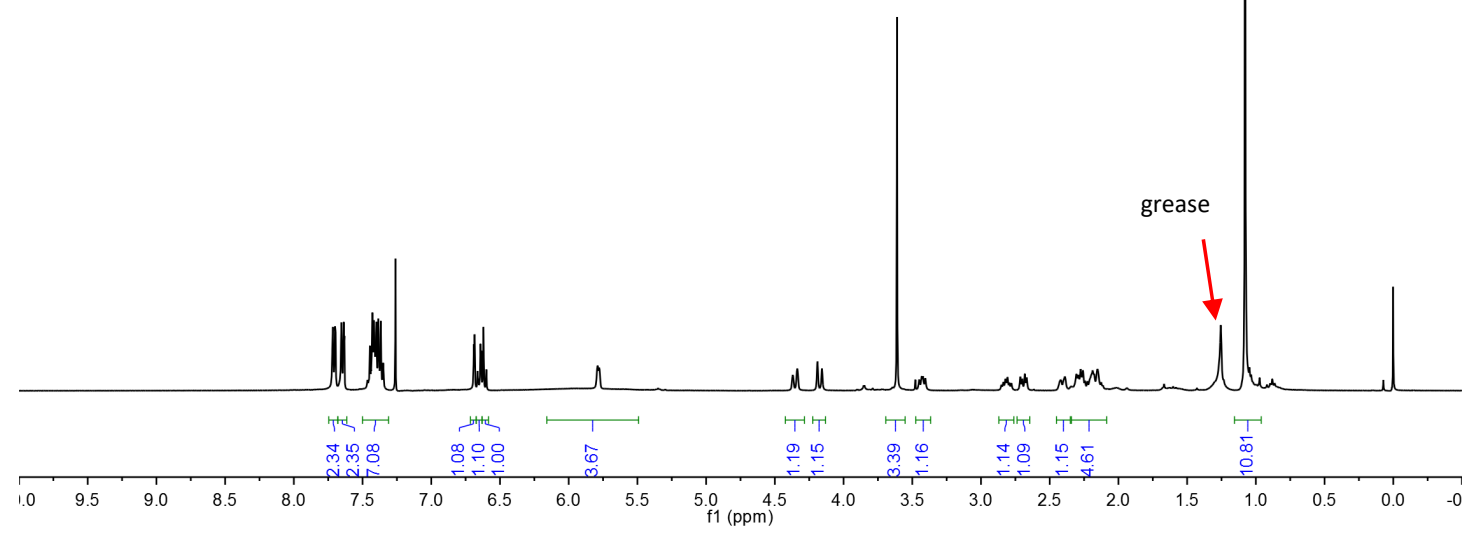

${ }^{13} \mathrm{C}\left\{{ }^{1} \mathrm{H}\right\}$ NMR $\left(\mathrm{CDCl}_{3}, 100 \mathrm{MHz}\right)$ of $\mathbf{9}$ :

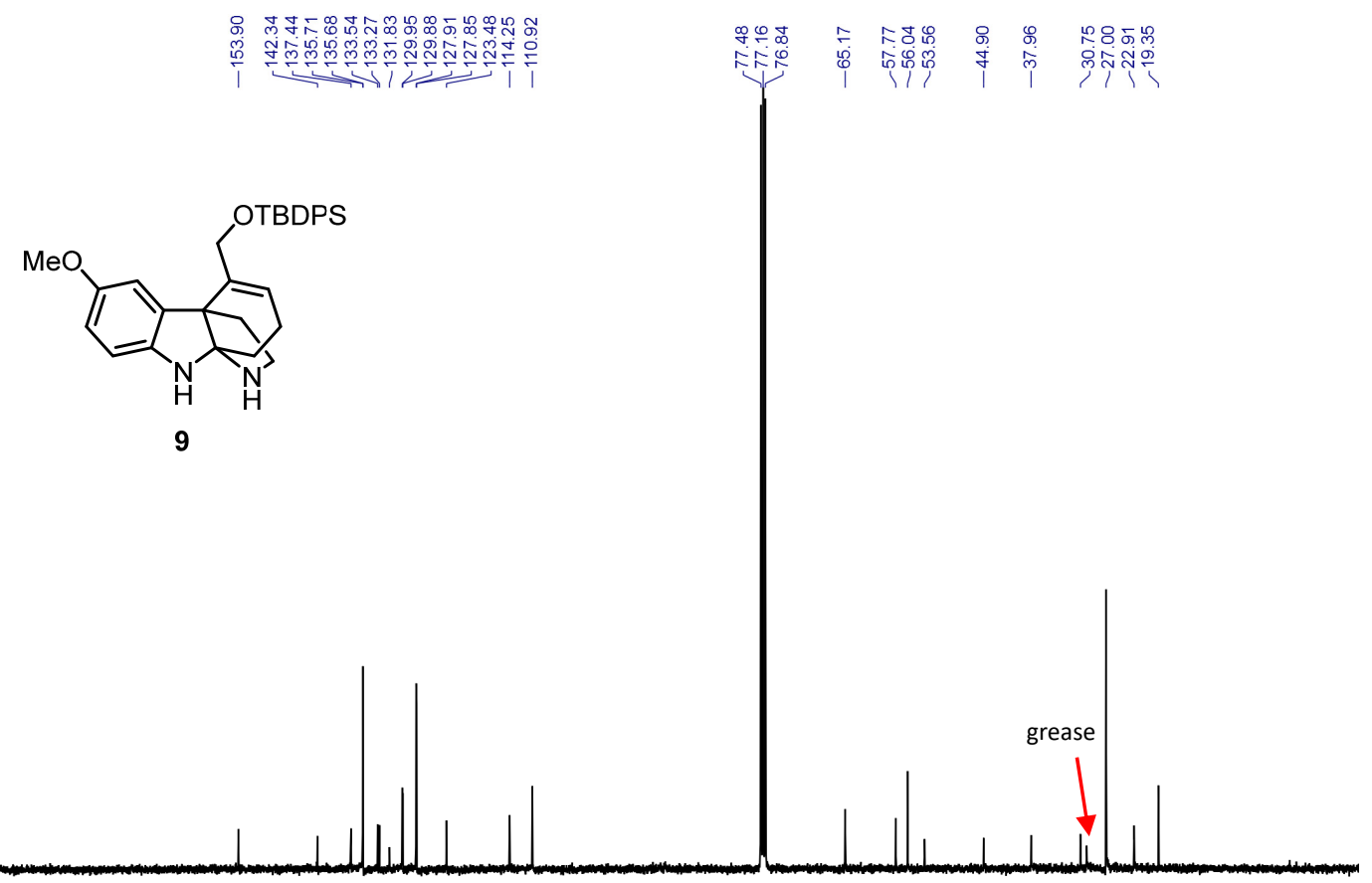

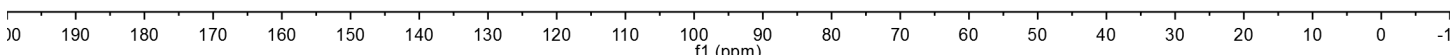


2D HSQC of 9 :

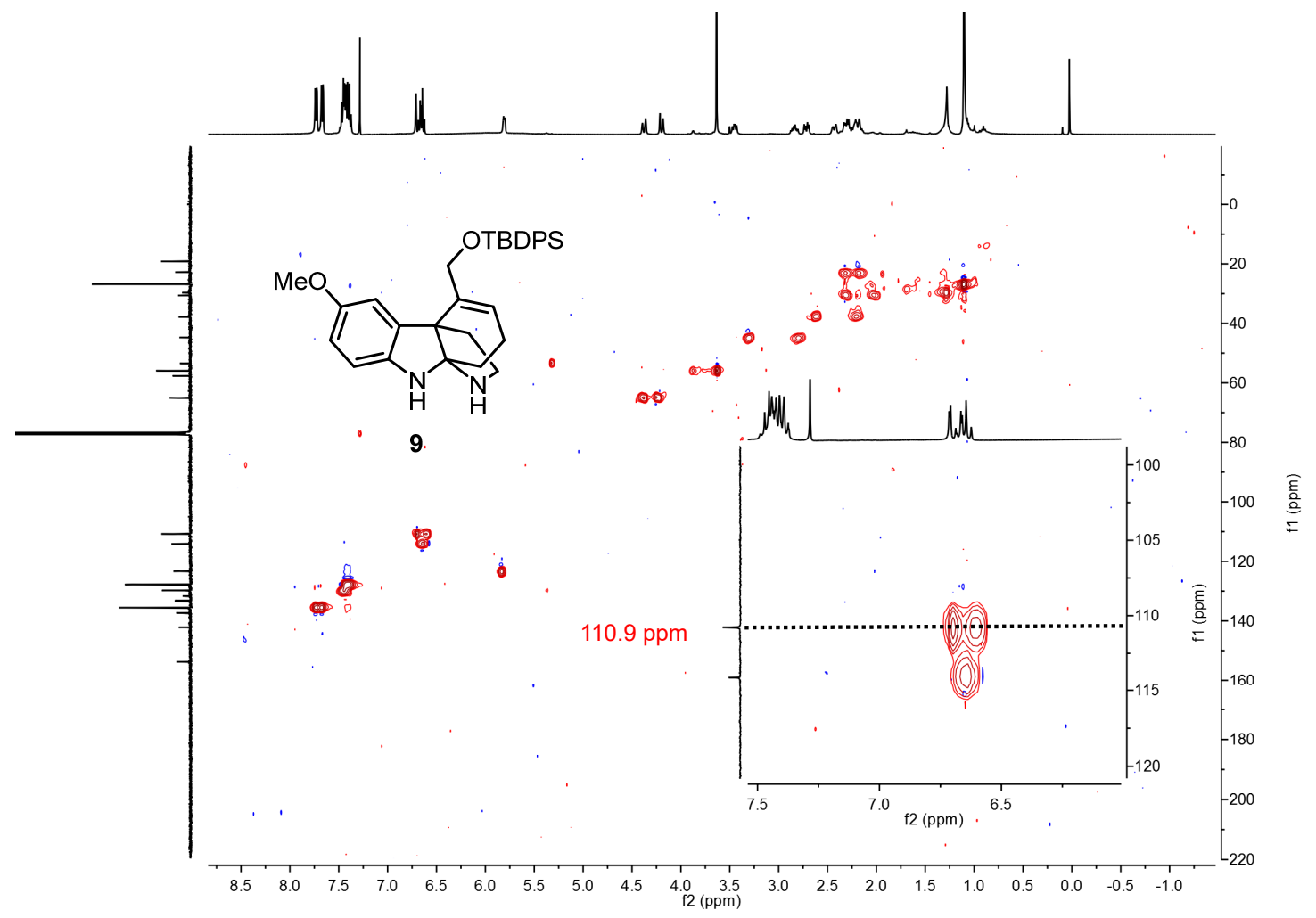


${ }^{1} \mathrm{H} \mathrm{NMR}\left(\mathrm{CDCl}_{3}, 400 \mathrm{MHz}\right)$ of $\mathbf{1 0}$ :

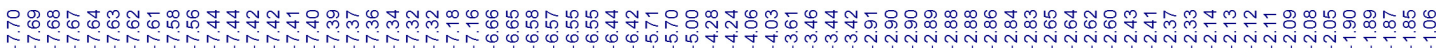
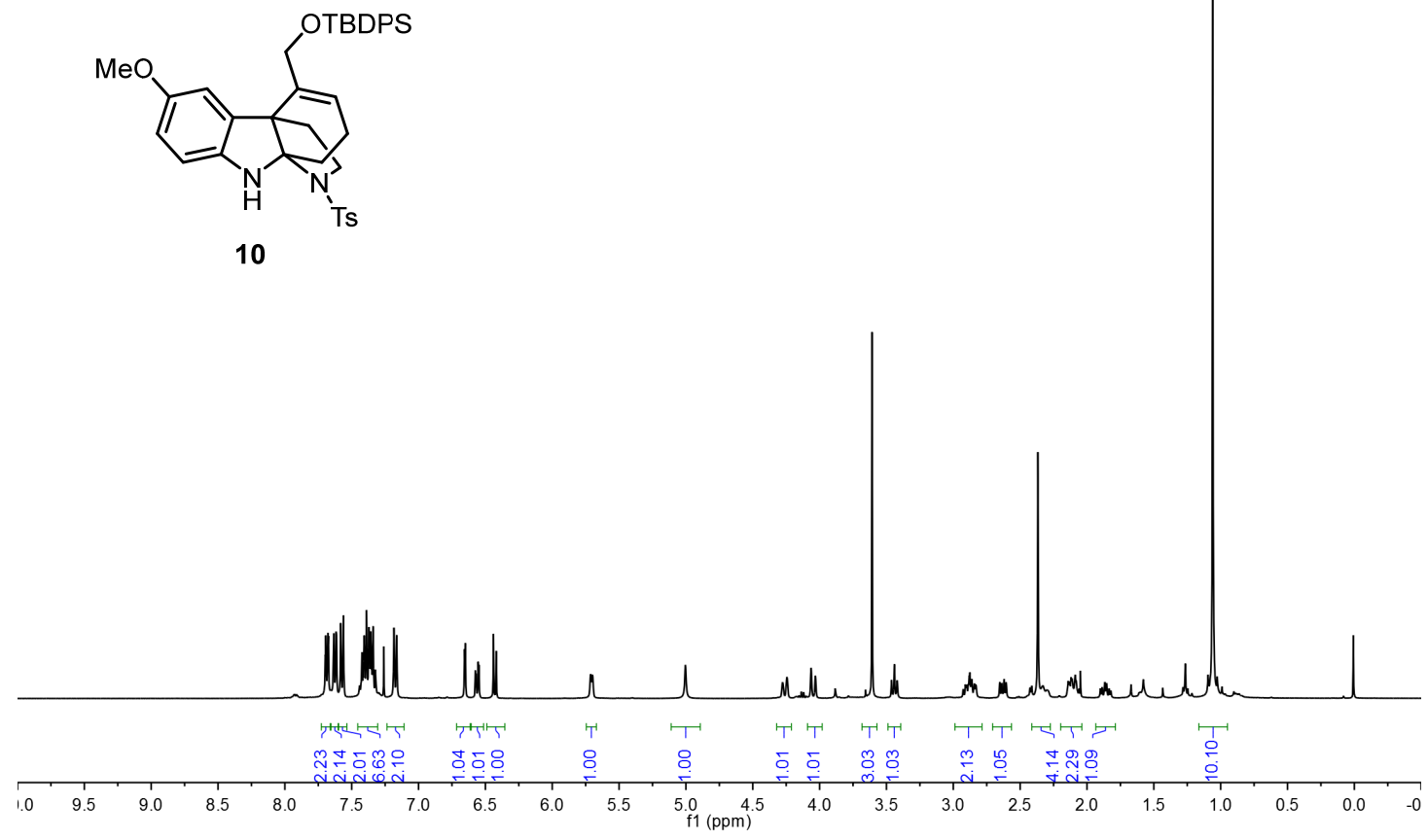

${ }^{13} \mathrm{C}\left\{{ }^{1} \mathrm{H}\right\}$ NMR $\left(\mathrm{CDCl}_{3}, 100 \mathrm{MHz}\right)$ of $\mathbf{1 0}$ :
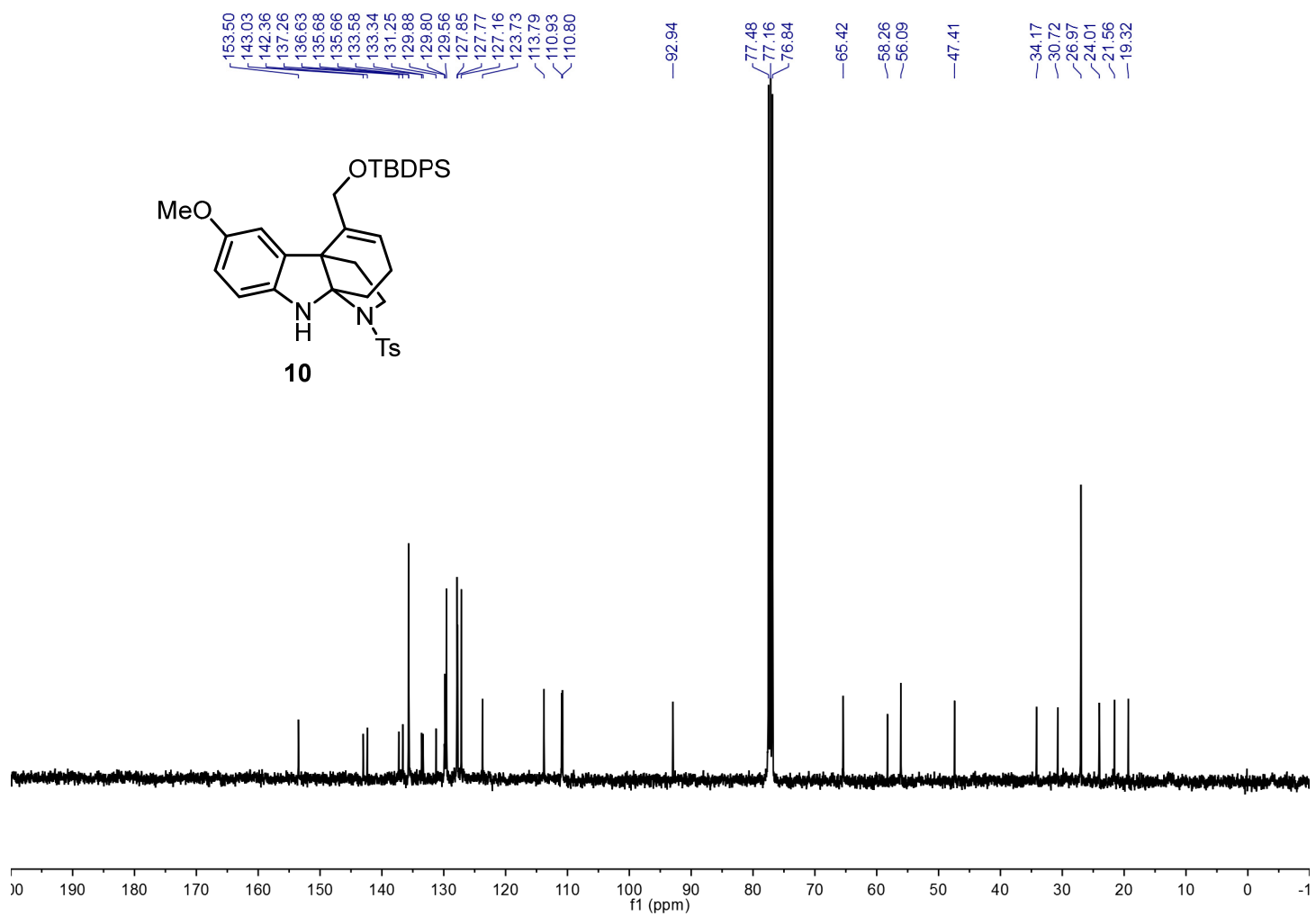
${ }^{1} \mathrm{H}$ NMR $\left(\mathrm{CDCl}_{3}, 400 \mathrm{MHz}\right)$ of $\mathbf{1 1}$ : $\sqrt{\underbrace{2}}$
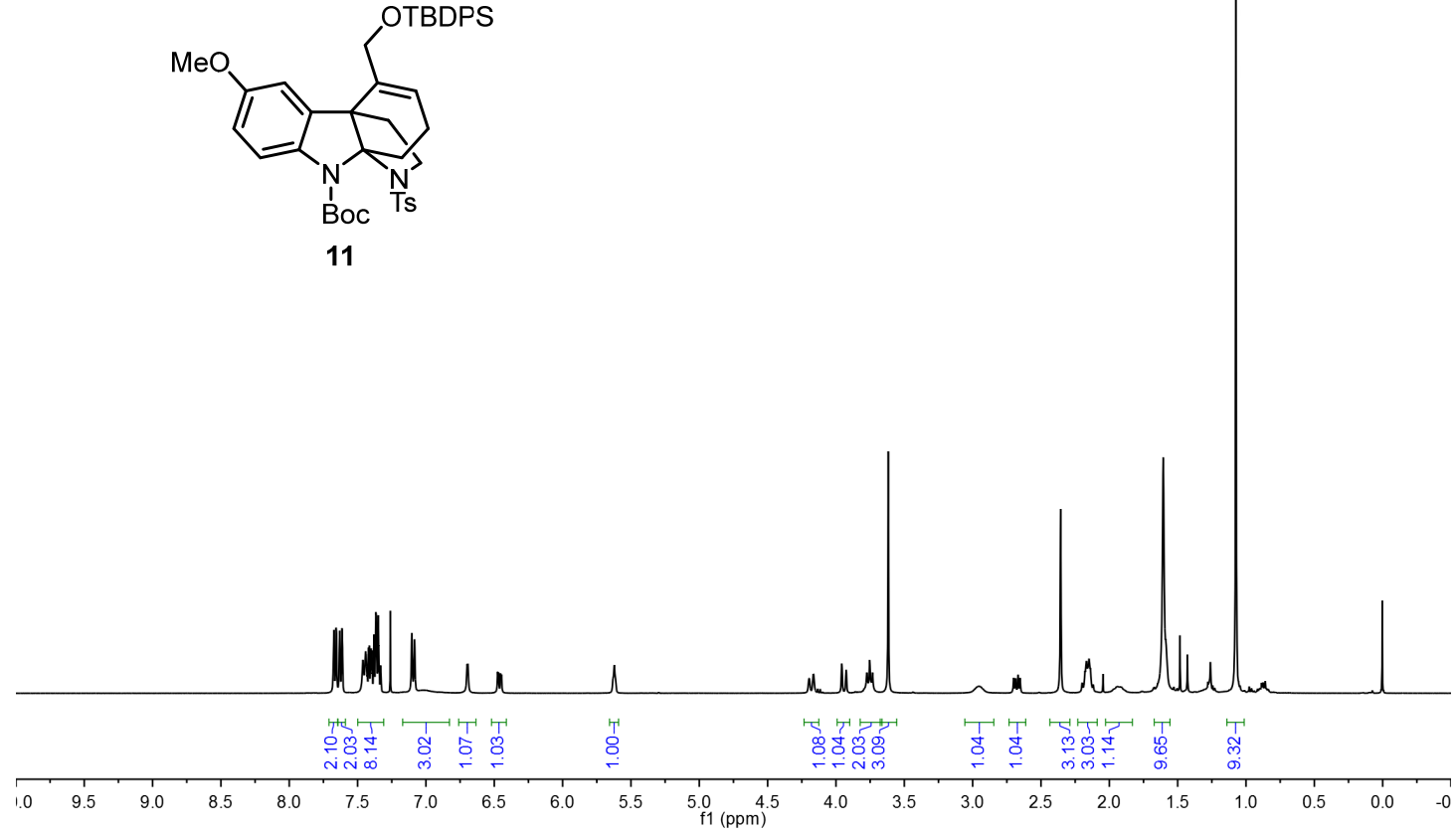

${ }^{13} \mathrm{C}\left\{{ }^{1} \mathrm{H}\right\} \mathrm{NMR}\left(\mathrm{CDCl}_{3}, 100 \mathrm{MHz}\right)$ of 11:
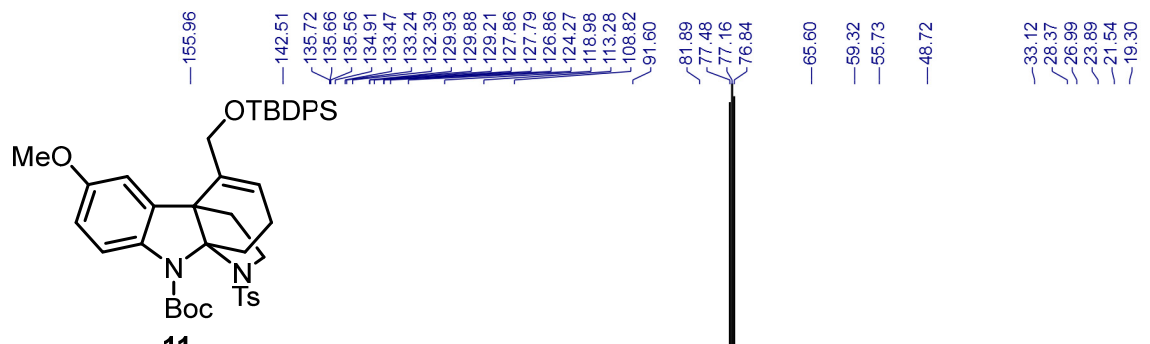

11

$\begin{array}{lllllllllll}10 & 190 & 180 & 170 & 160 & 150 & 140 & 130 & 120 & 110 & 100 \\ \mathrm{f} 1(\mathrm{ppm})\end{array}$ 
2D HSQC of 11:

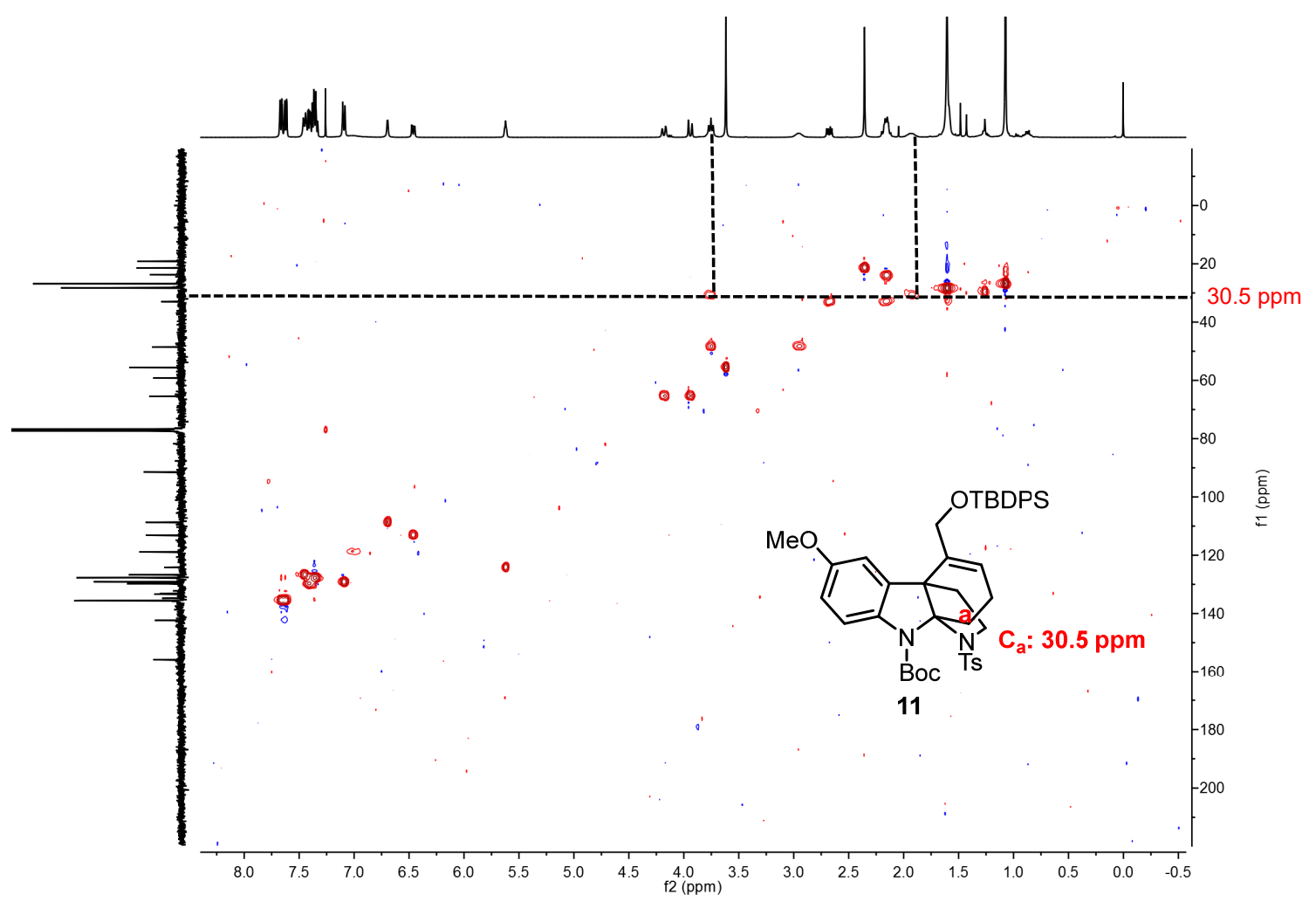

2D HMBC of 11:

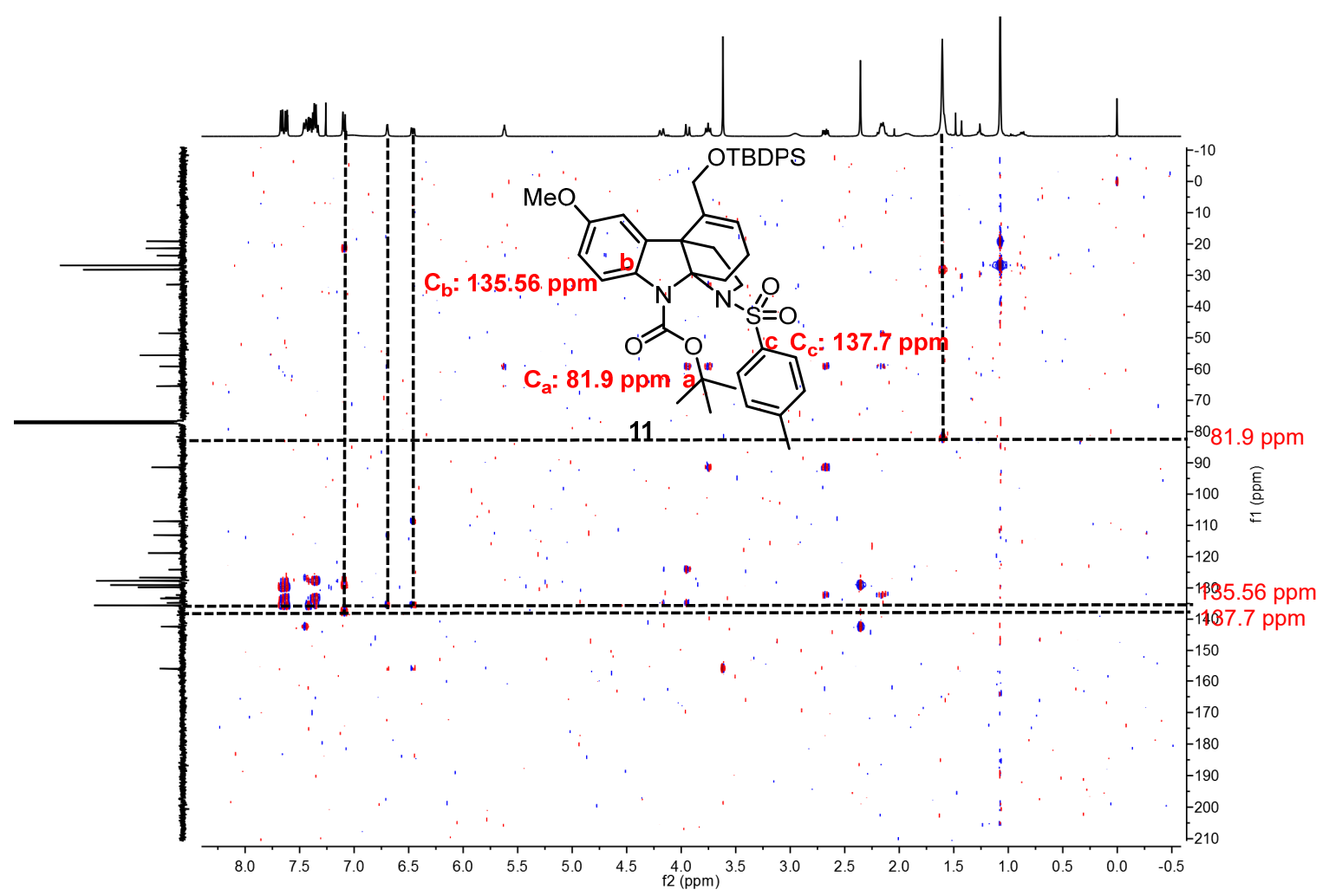


${ }^{1} \mathrm{H} \mathrm{NMR}\left(\mathrm{CDCl}_{3}, 600 \mathrm{MHz}\right)$ of $\mathbf{1 2}$ :

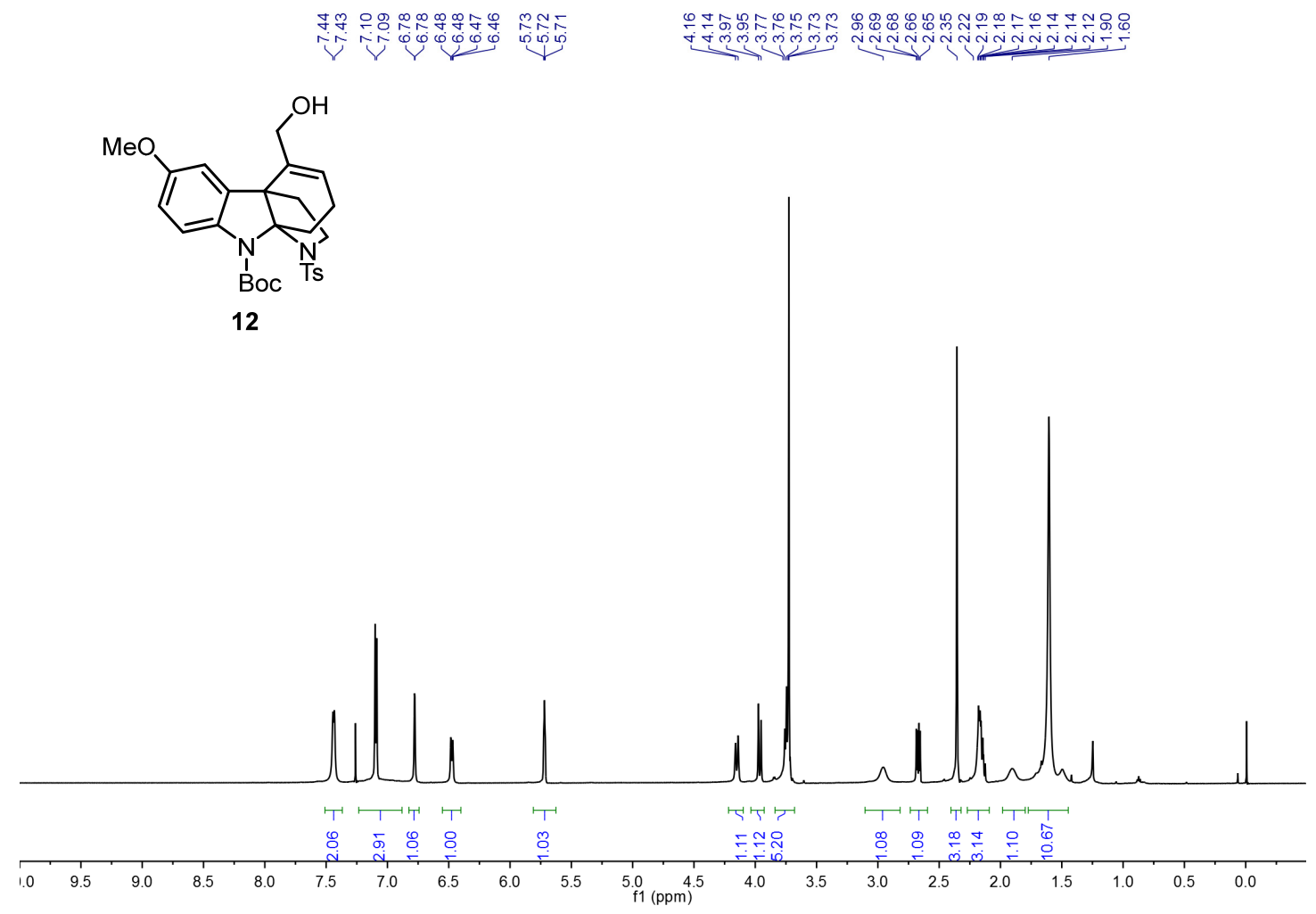

${ }^{13} \mathrm{C}\left\{{ }^{1} \mathrm{H}\right\}$ NMR $\left(\mathrm{CDCl}_{3}, 150 \mathrm{MHz}\right)$ of $\mathbf{1 2}$ :

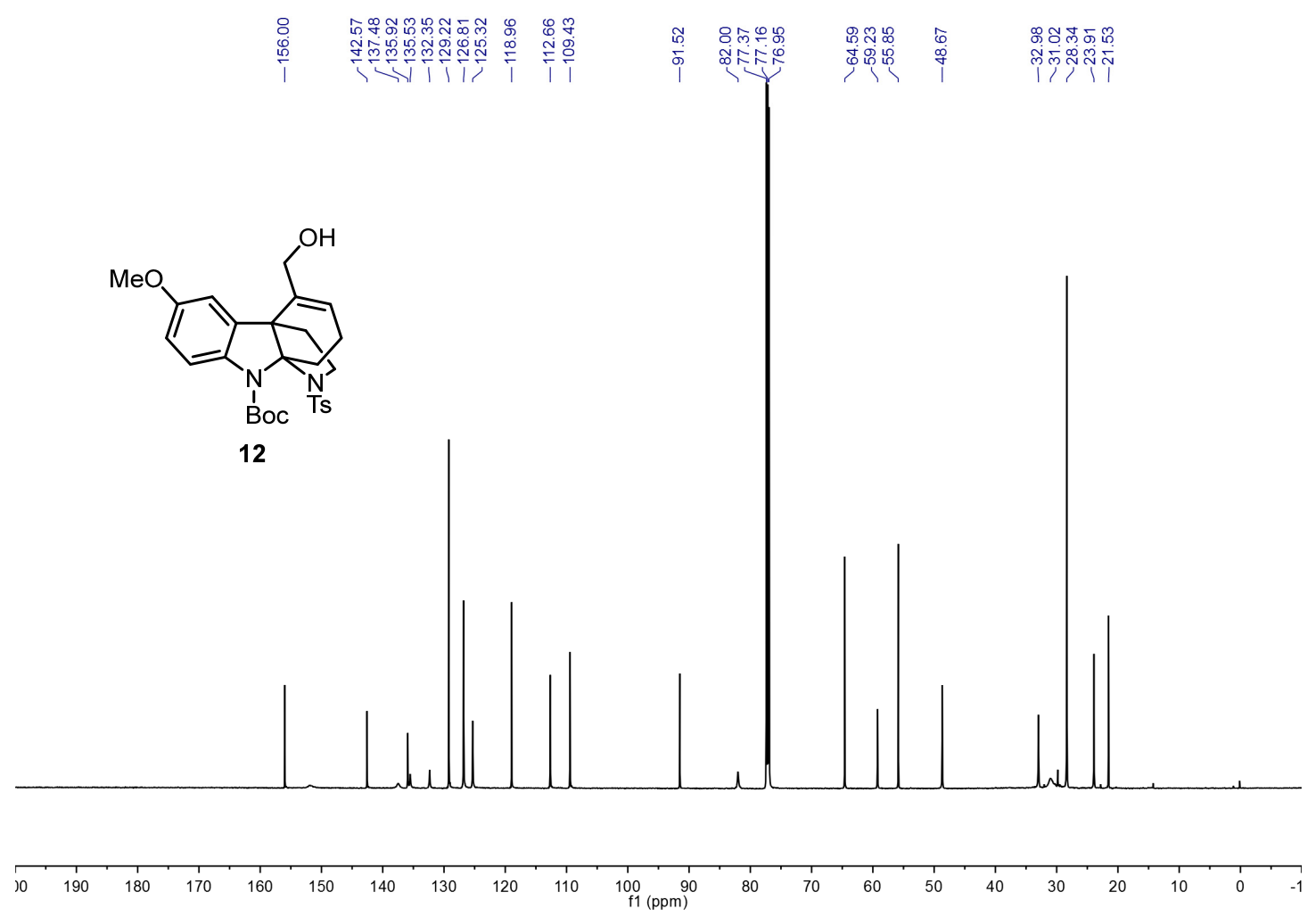


Comparison of the ${ }^{1} \mathrm{H}$ and ${ }^{13} \mathrm{C}$ NMR spectra of synthesized 12 and those reported in literature: ${ }^{1}$
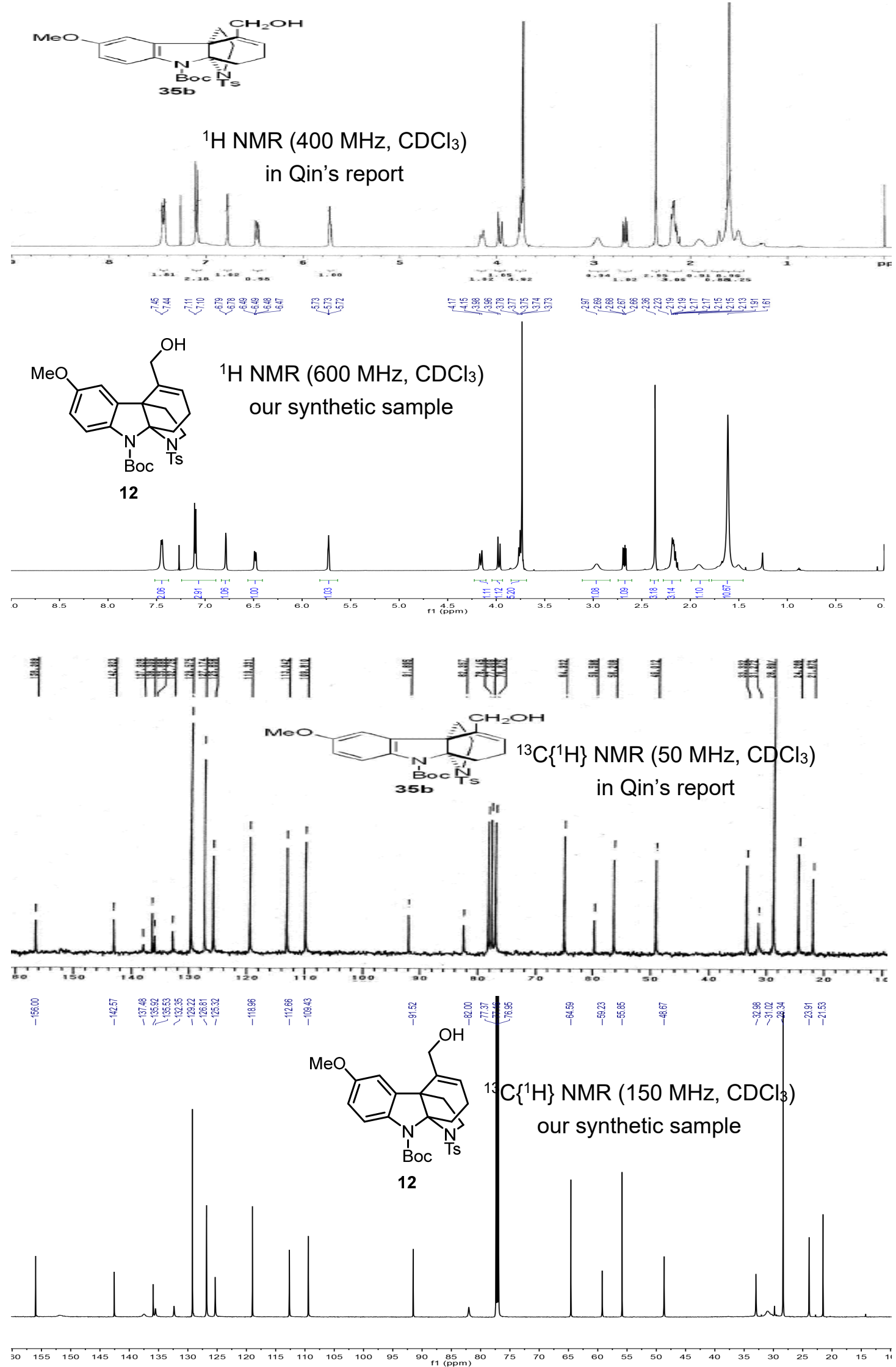
${ }^{1} \mathrm{H} \mathrm{NMR}\left(\mathrm{CDCl}_{3}, 400 \mathrm{MHz}\right)$ of $\mathbf{S 2 0}$ :

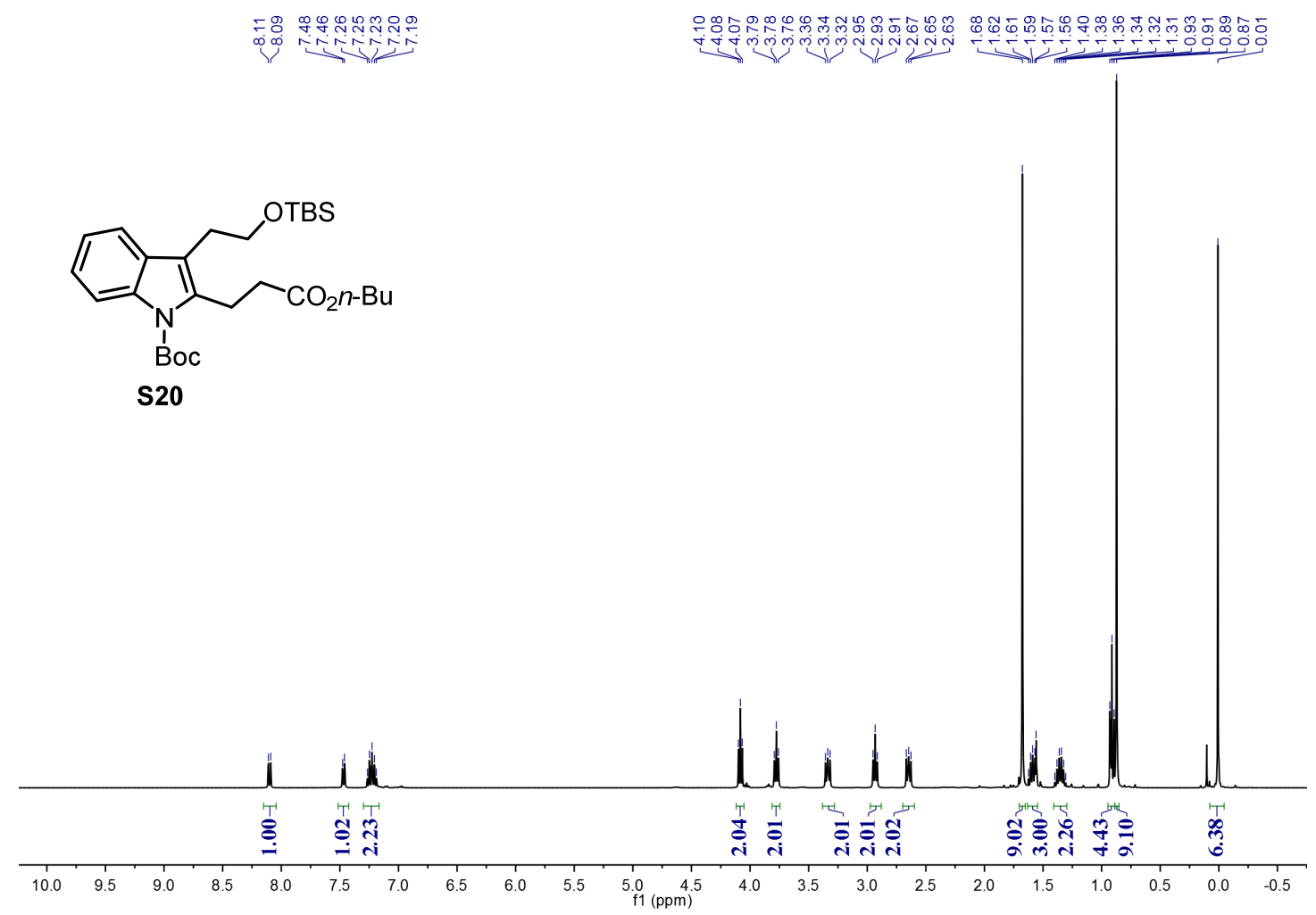

${ }^{13} \mathrm{C}\left\{{ }^{1} \mathrm{H}\right\}$ NMR $\left(\mathrm{CDCl}_{3}, 100 \mathrm{MHz}\right)$ of $\mathbf{S 2 0}$ :

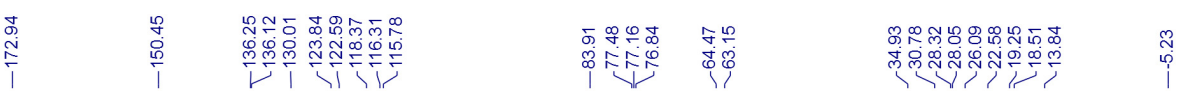

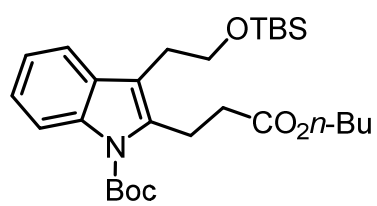

S20

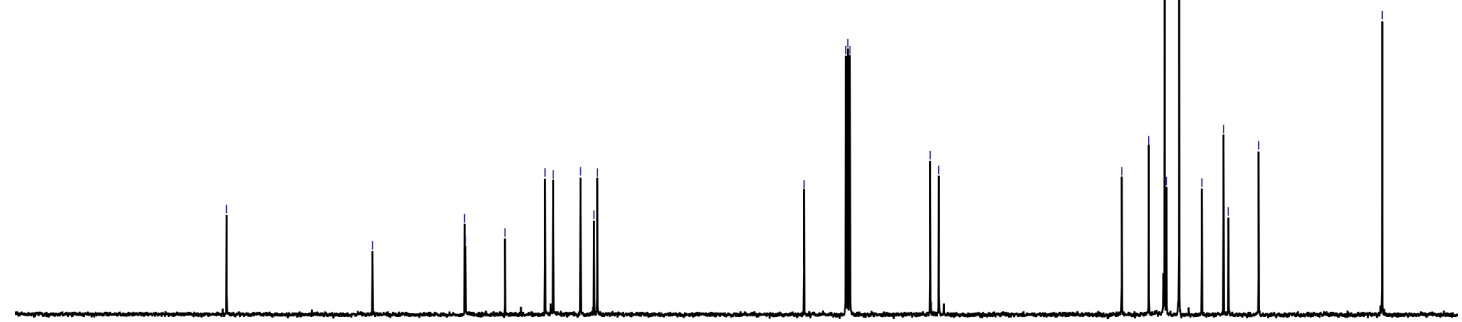

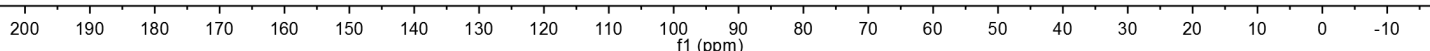


${ }^{1} \mathrm{H}$ NMR $\left(\mathrm{CDCl}_{3}, 400 \mathrm{MHz}\right)$ of $\mathbf{S 2 2}$ :

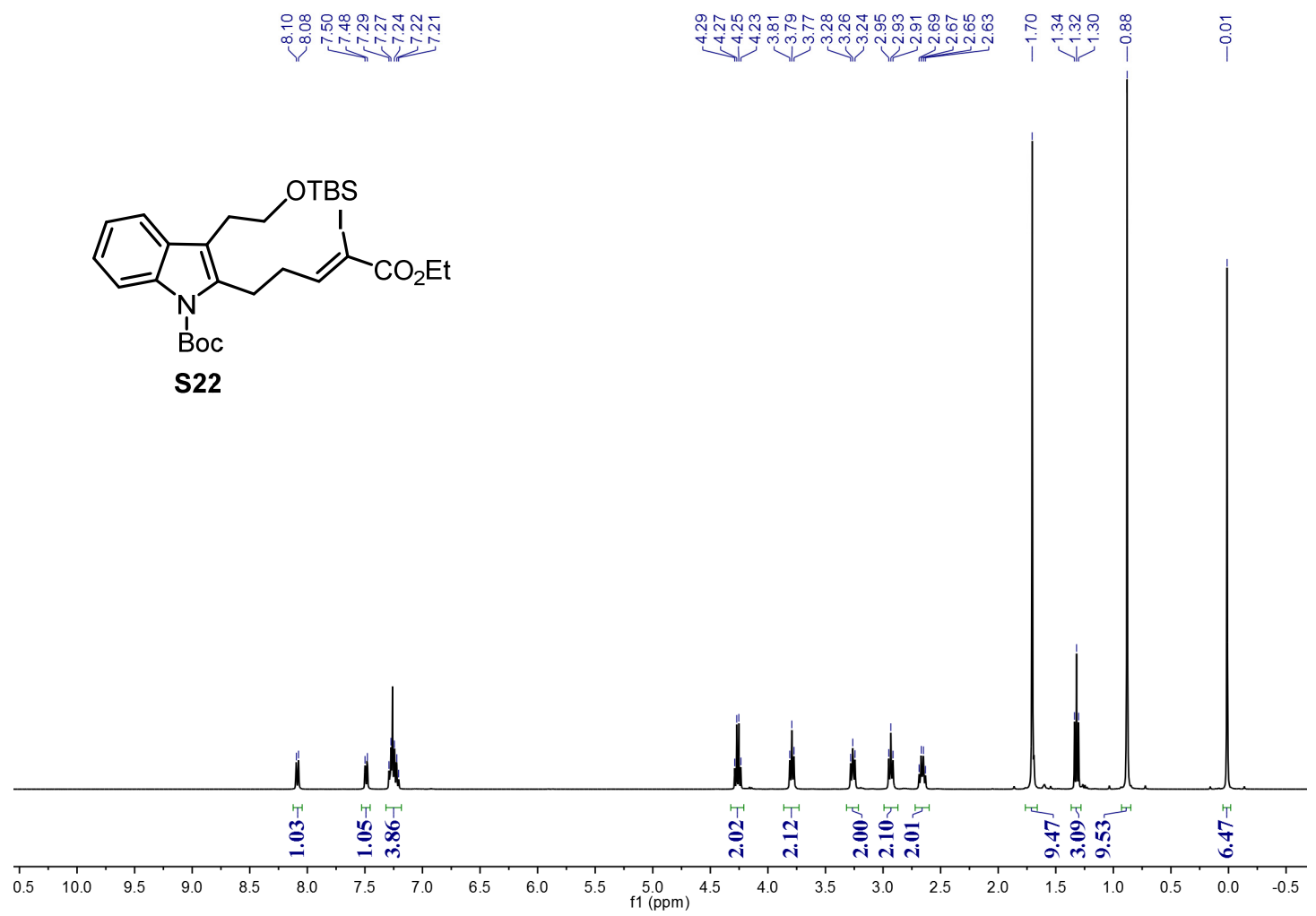

${ }^{13} \mathrm{C}\left\{{ }^{1} \mathrm{H}\right\}$ NMR $\left(\mathrm{CDCl}_{3}, 100 \mathrm{MHz}\right)$ of $\mathbf{S 2 2}$ :

广

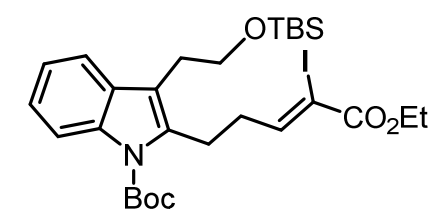

S22

$\begin{array}{lllllllllll}200 & 190 & 180 & 170 & 160 & 150 & 140 & 130 & 120 & 110 & \underset{f 1}{100} 90 \\ \mathrm{f} 1(\mathrm{ppm}) & \end{array}$ 
${ }^{1} \mathrm{H} \mathrm{NMR}\left(\mathrm{CDCl}_{3}, 400 \mathrm{MHz}\right)$ of $\mathbf{1 p}$ :

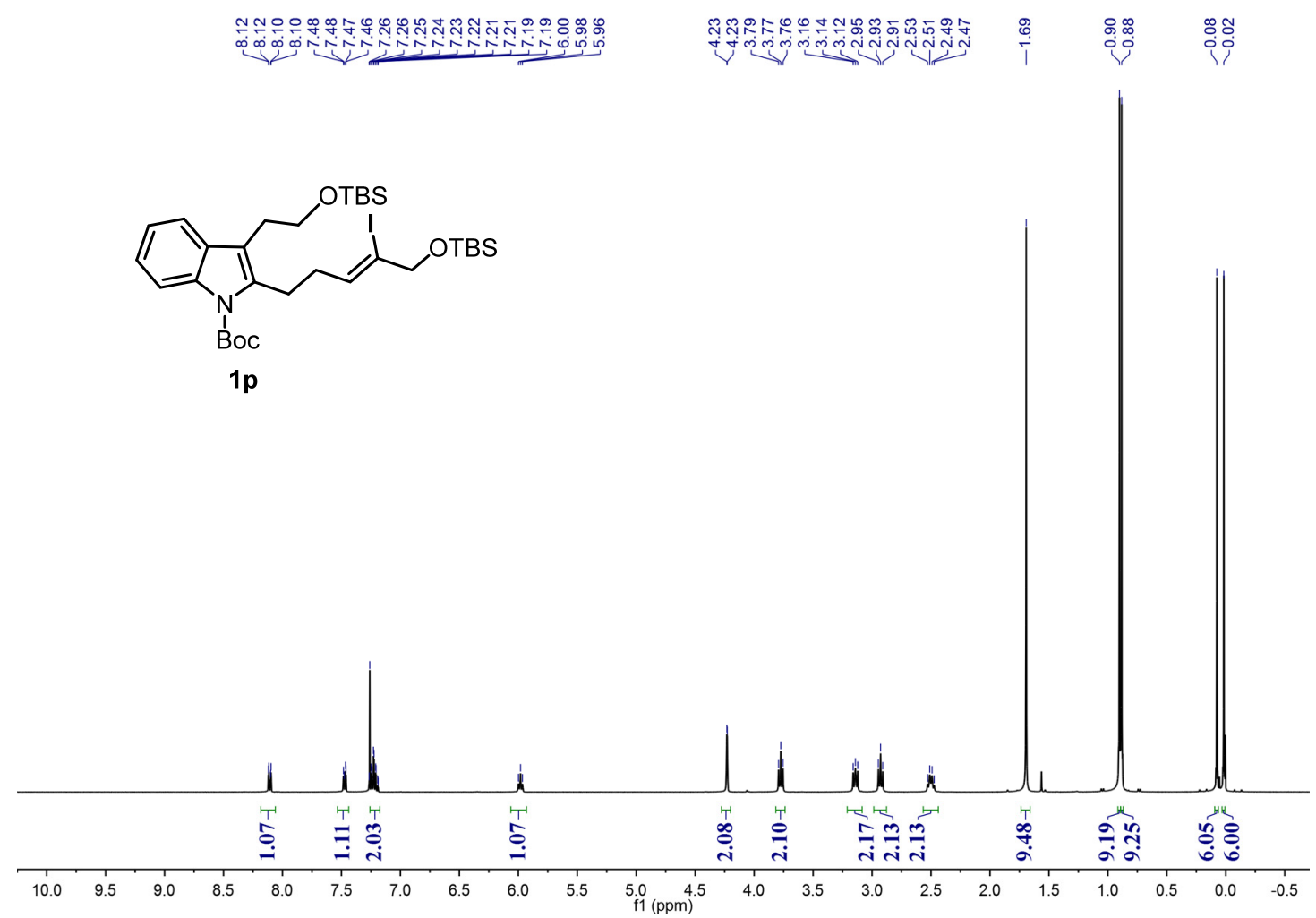

${ }^{13} \mathrm{C}\left\{{ }^{1} \mathrm{H}\right\} \mathrm{NMR}\left(\mathrm{CDCl}_{3}, 100 \mathrm{MHz}\right)$ of $\mathbf{1 p}:$
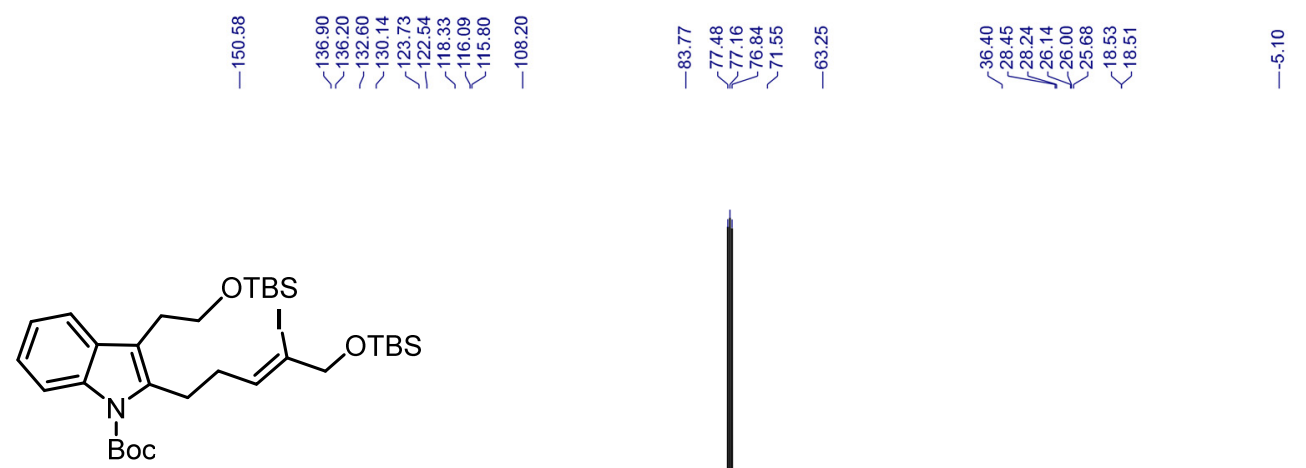

$1 p$

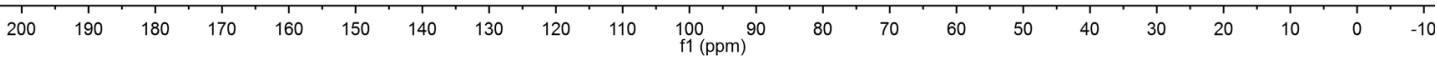


2 D HSQC NMR of 1p:

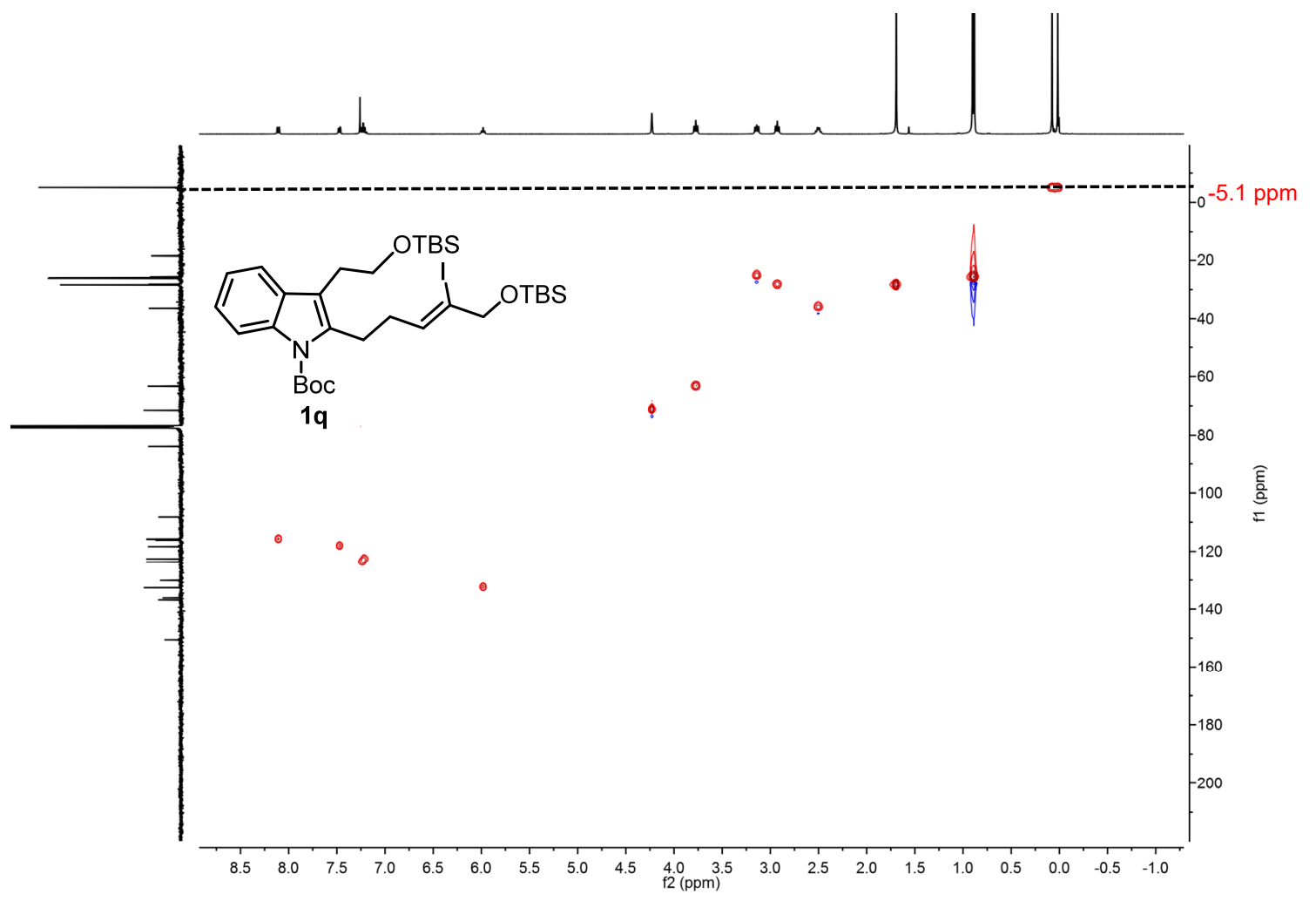


${ }^{1} \mathrm{H} \mathrm{NMR}\left(\mathrm{CDCl}_{3}, 400 \mathrm{MHz}\right)$ of 1q:

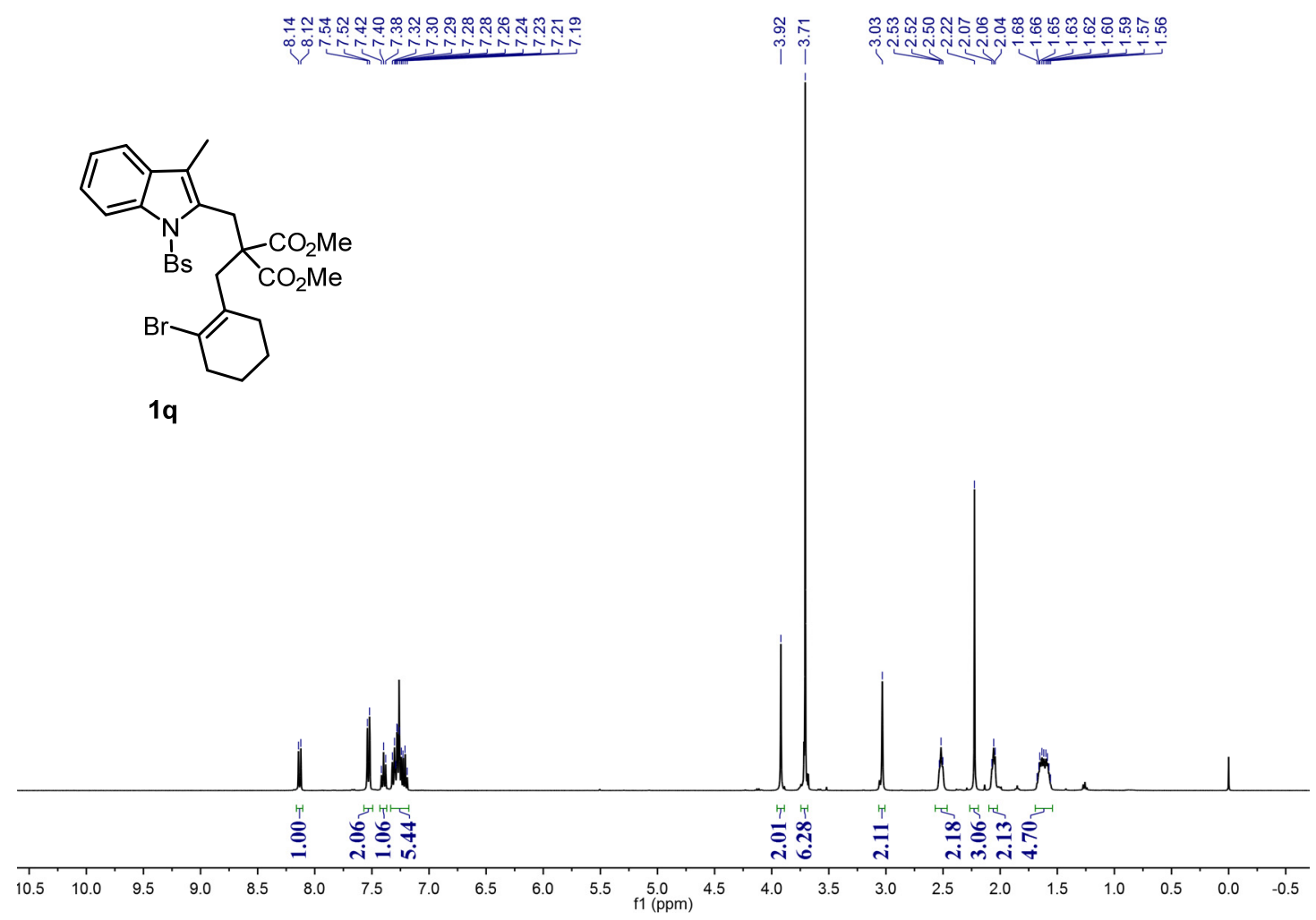

${ }^{13} \mathrm{C}\left\{{ }^{1} \mathrm{H}\right\}$ NMR $\left(\mathrm{CDCl}_{3}, 100 \mathrm{MHz}\right)$ of $\mathbf{1 q}$ :

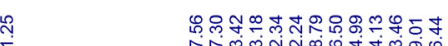

लिल凶ल

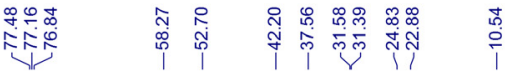

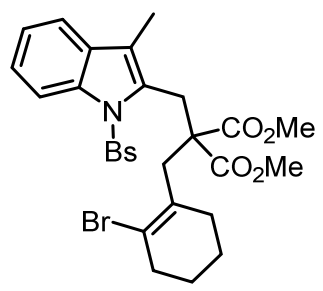

$1 q$

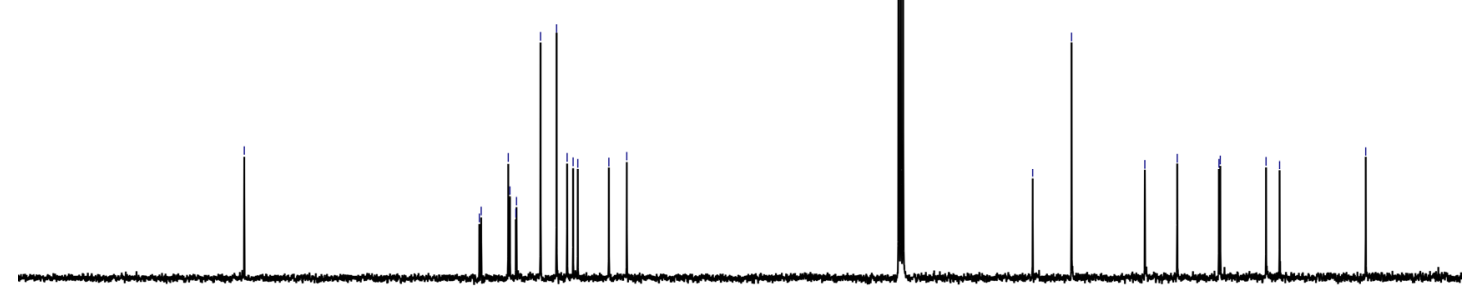

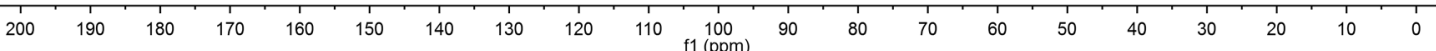


${ }^{1} \mathrm{H}$ NMR $\left(\mathrm{CDCl}_{3}, 400 \mathrm{MHz}\right)$ of $\mathbf{2 p}$ : б.

$8.23 \% \mathrm{nOe}$

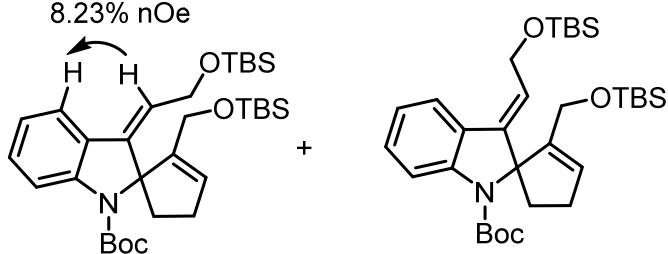

Boc

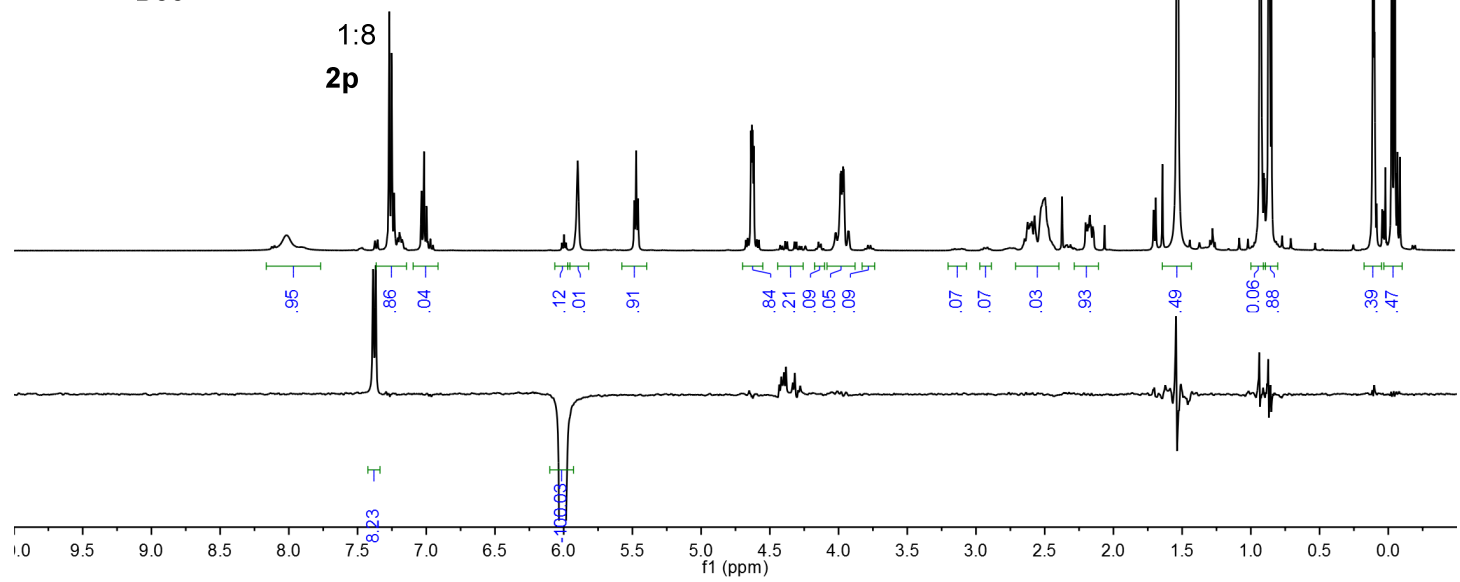

${ }^{1} \mathrm{H}$ NMR $\left(\mathrm{CDCl}_{3}, 400 \mathrm{MHz}\right)$ of $\mathbf{2 p}$ :

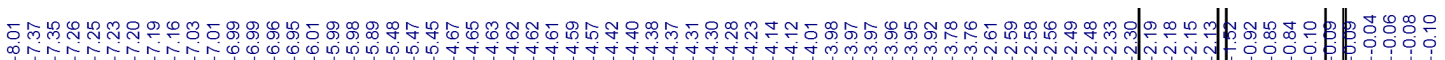

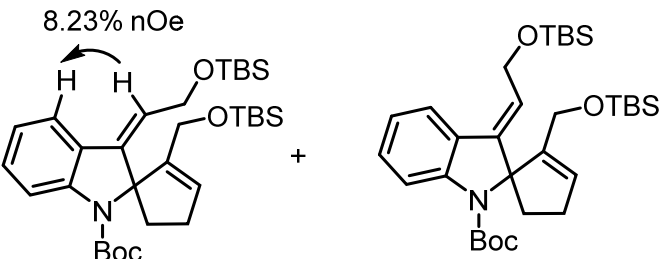

$1: 8$

$2 p$
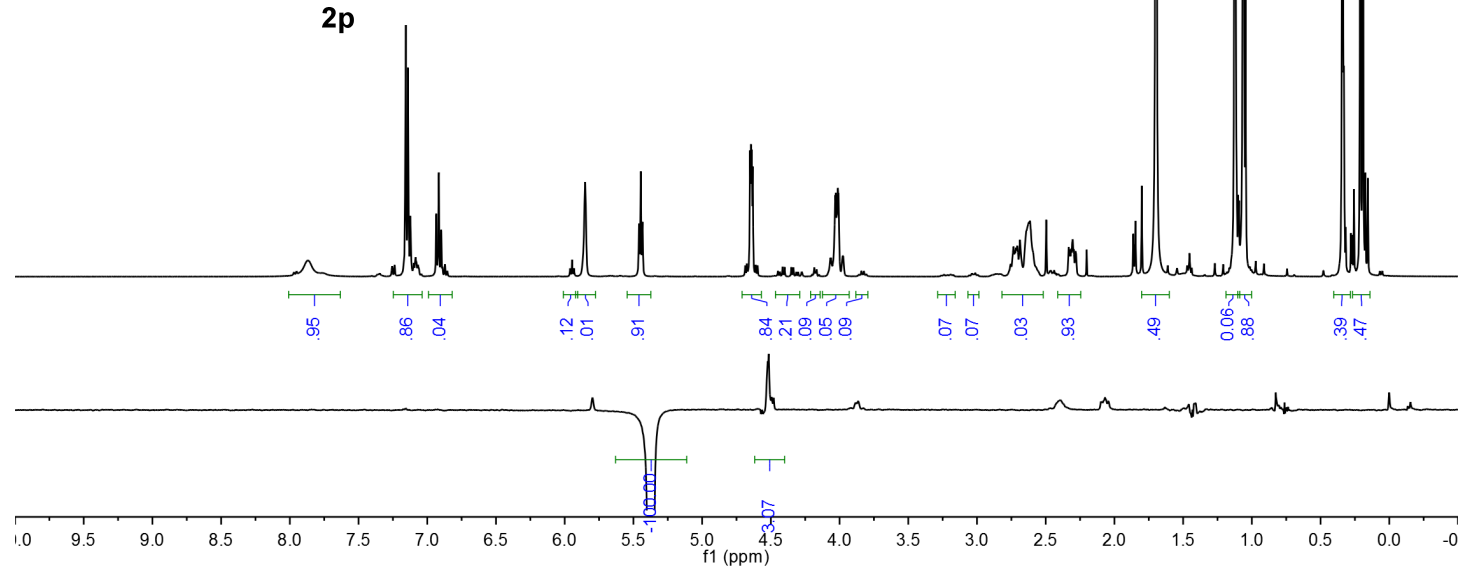
${ }^{13} \mathrm{C}\left\{{ }^{1} \mathrm{H}\right\}$ NMR $\left(\mathrm{CDCl}_{3}, 100 \mathrm{MHz}\right)$ of $\mathbf{2 p}$ :

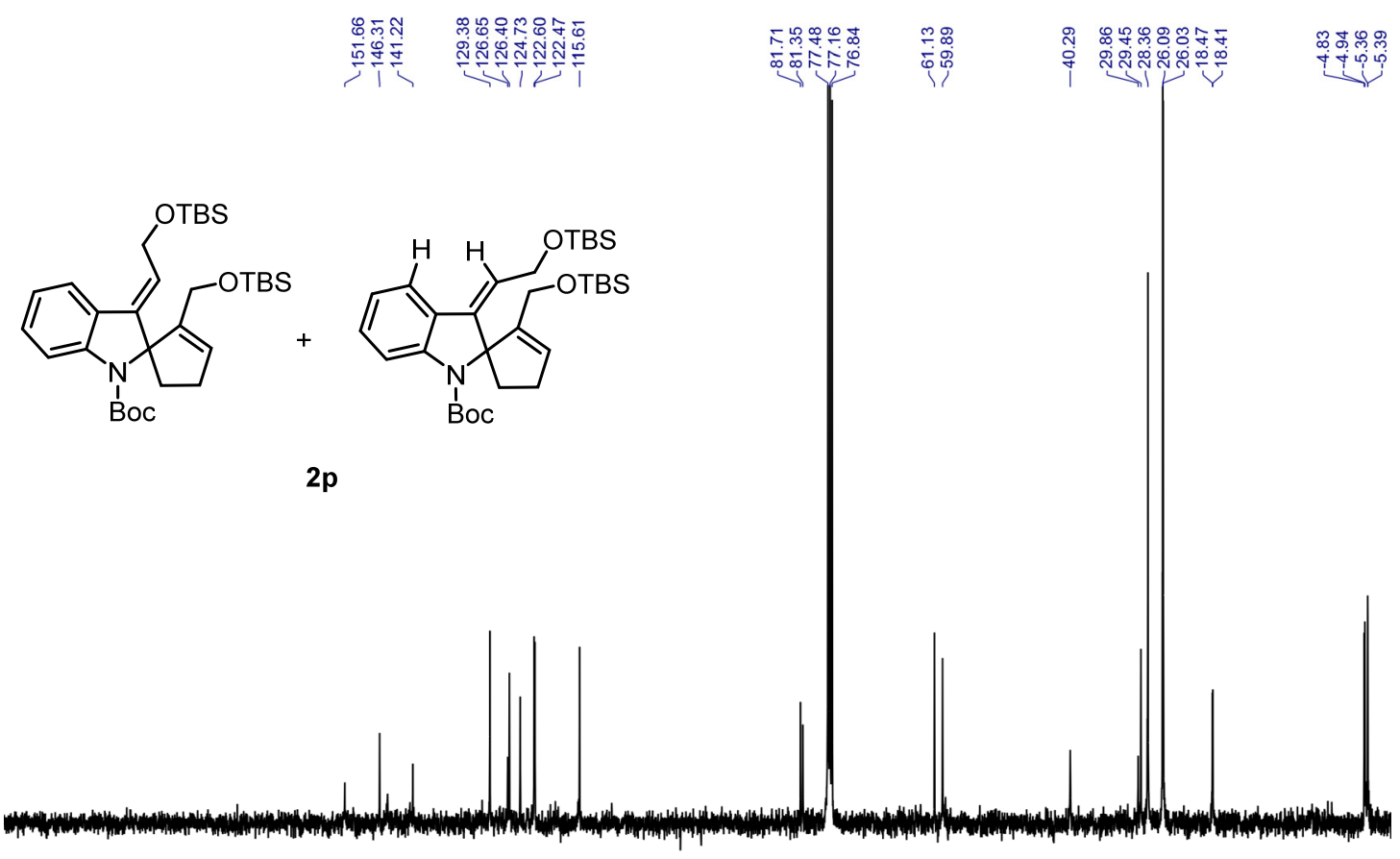

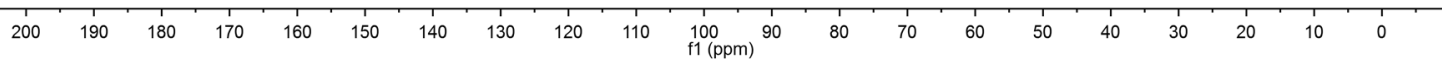


${ }^{1} \mathrm{H} \mathrm{NMR}\left(\mathrm{CDCl}_{3}, 400 \mathrm{MHz}\right)$ of $\mathbf{2 q}:$

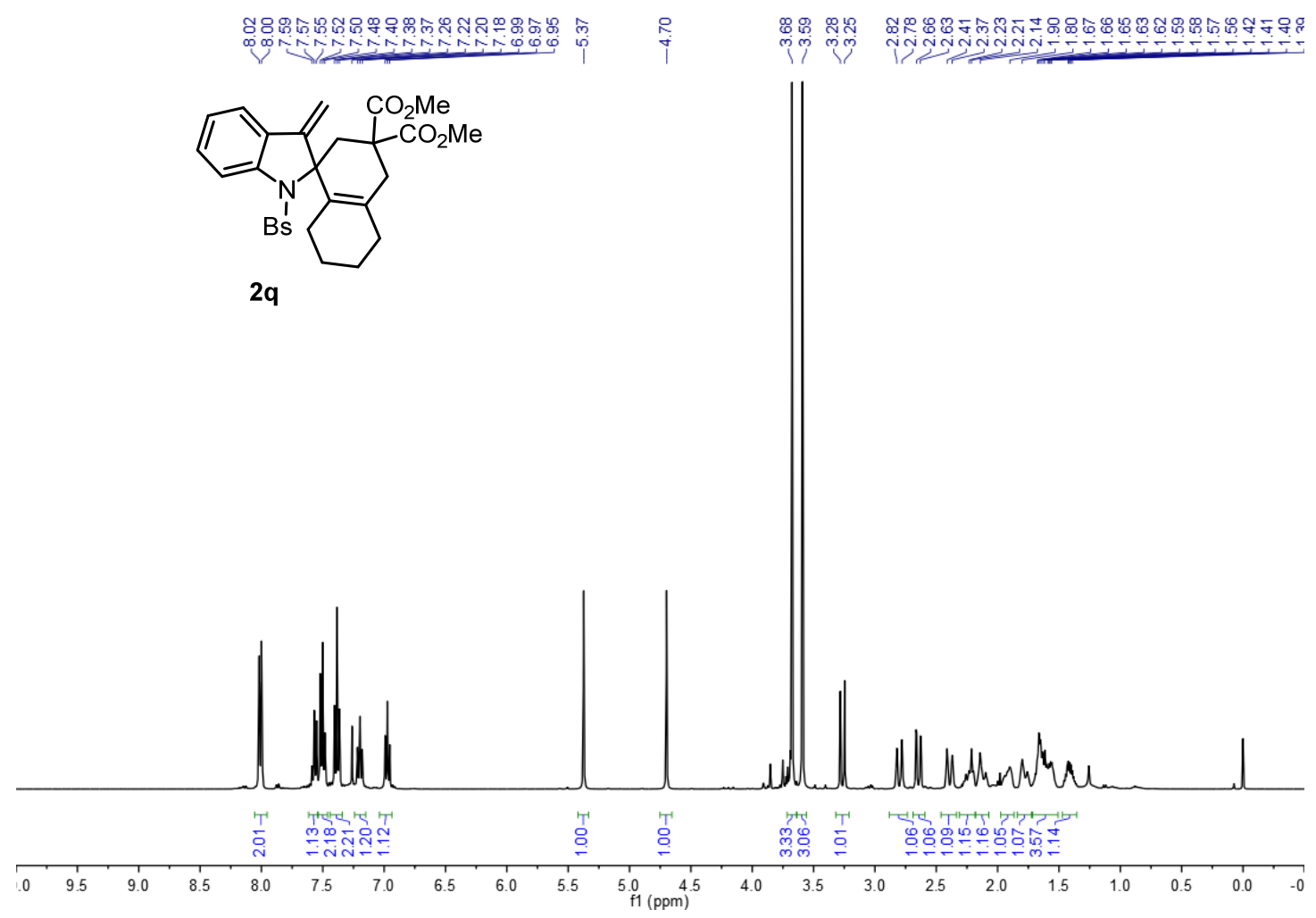

${ }^{13} \mathrm{C}\left\{{ }^{1} \mathrm{H}\right\} \mathrm{NMR}\left(\mathrm{CDCl}_{3}, 100 \mathrm{MHz}\right)$ of $\mathbf{2 q}:$

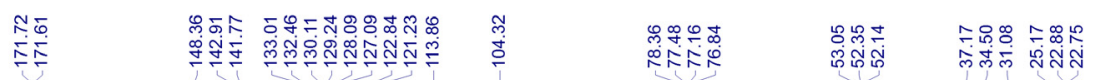

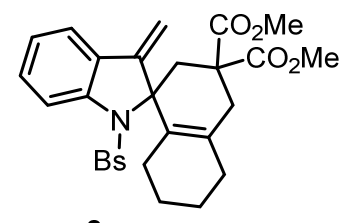

$2 q$

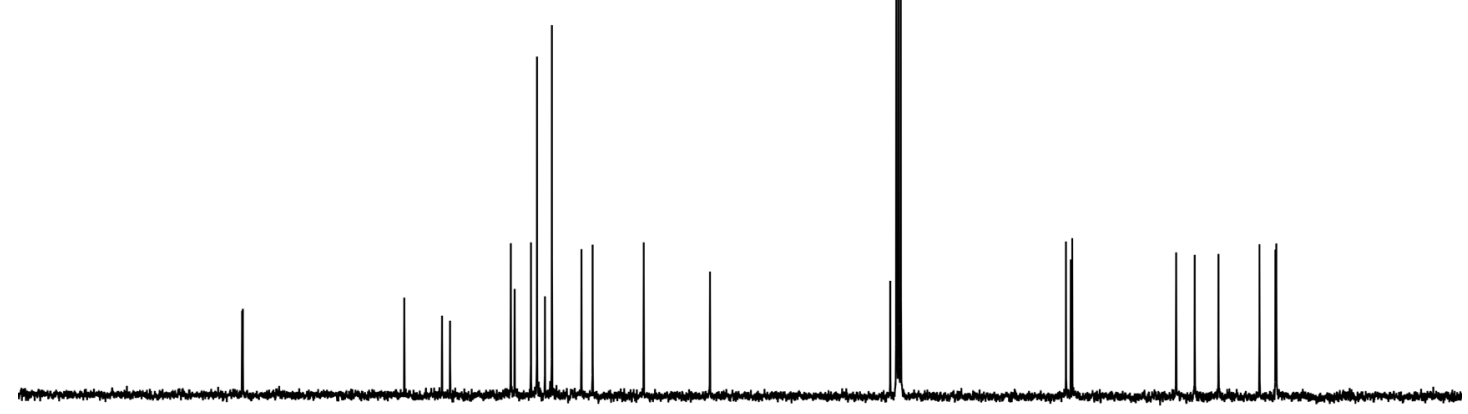

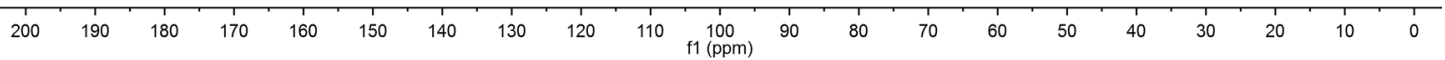




\section{References}

(1) Zhang, M.; Huang, X.; Shen, L.; Qin, Y. Total Synthesis of the Akuammiline Alkaloid ( \pm )Vincorine. J. Am. Chem. Soc. 2009, 131, 6013. 